
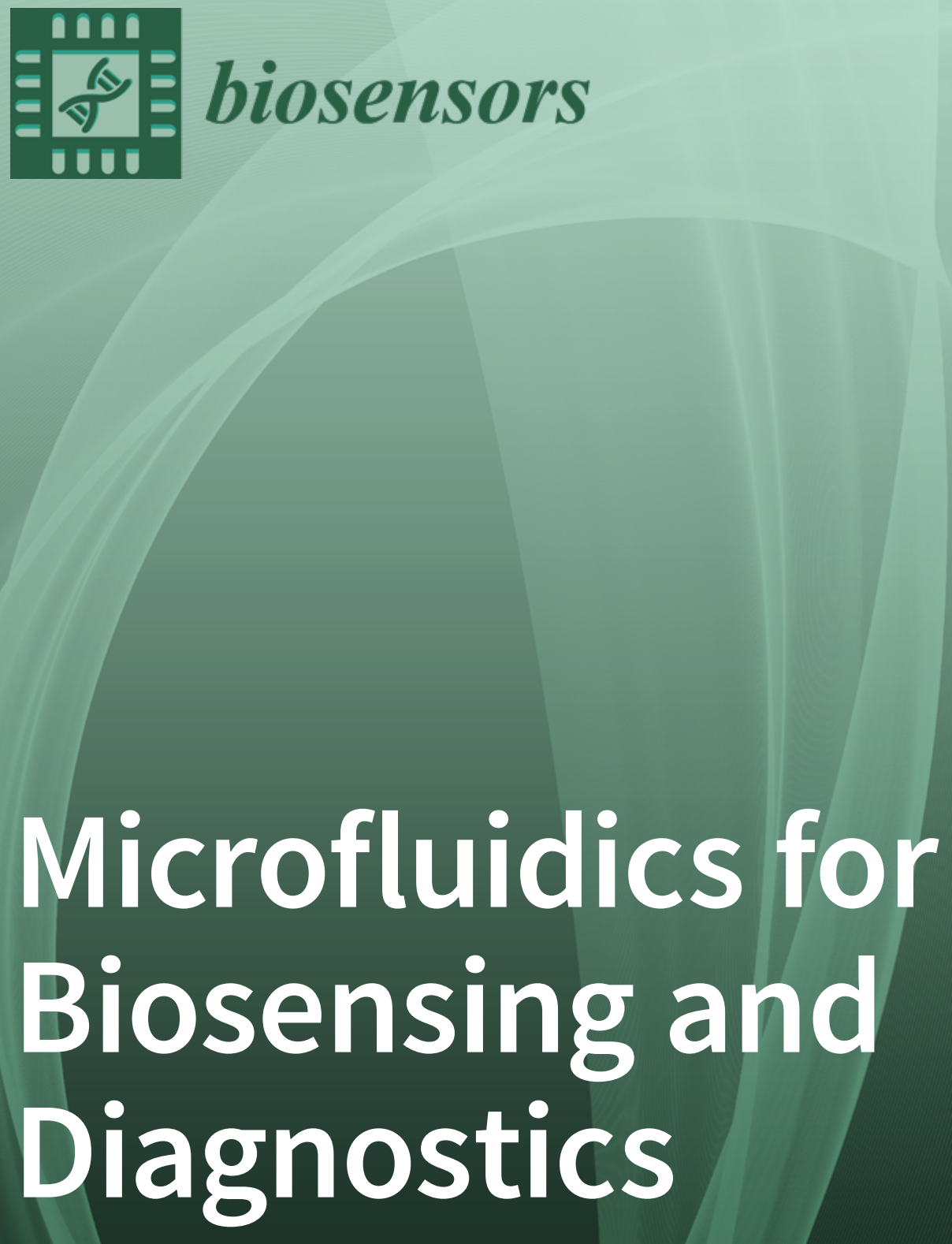

Edited by David W. Inglis, Majid Ebrahimi Warkiani, Mohammad A. Qasaimeh and Weiqiang Chen Printed Edition of the Special Issue Published in Biosensors 


\section{Microfluidics for Biosensing and Diagnostics}





\section{Microfluidics for Biosensing and Diagnostics}

Editors

David W. Inglis

Majid Ebrahimi Warkiani

Mohammad A. Qasaimeh

Weiqiang Chen 
Editors

David W. Inglis

Macquarie University

Australia

Weiqiang Chen

New York University

USA

Editorial Office

MDPI

St. Alban-Anlage 66

4052 Basel, Switzerland
Majid Ebrahimi Warkiani

University of Technology Sydney

Australia
Mohammad A. Qasaimeh

New York University Abu Dhabi

UAE

This is a reprint of articles from the Special Issue published online in the open access journal Biosensors (ISSN 2079-6374) (available at: https://www.mdpi.com/journal/biosensors/special_ issues/Microfluidics_Biosensing_Diagnostics).

For citation purposes, cite each article independently as indicated on the article page online and as indicated below:

LastName, A.A.; LastName, B.B.; LastName, C.C. Article Title. Journal Name Year, Volume Number, Page Range.

ISBN 978-3-0365-0062-1 (Hbk)

ISBN 978-3-0365-0063-8 (PDF)

(C) 2020 by the authors. Articles in this book are Open Access and distributed under the Creative Commons Attribution (CC BY) license, which allows users to download, copy and build upon published articles, as long as the author and publisher are properly credited, which ensures maximum dissemination and a wider impact of our publications.

The book as a whole is distributed by MDPI under the terms and conditions of the Creative Commons license CC BY-NC-ND. 


\section{Contents}

About the Editors $\ldots \ldots \ldots \ldots \ldots \ldots \ldots \ldots \ldots \ldots \ldots$

Preface to "Microfluidics for Biosensing and Diagnostics" $\ldots \ldots \ldots \ldots \ldots$ ix

Zain Hayat, Nizar Bchellaoui, Claire Deo, Rémi Métivier, Nicolas Bogliotti, Juan Xie, Malcolm Buckle and Abdel I. El Abed

Fast Active Merging of Microdroplets in Microfluidic Chambers Driven by Photo-Isomerisation of Azobenzene Based Surfactants

Reprinted from: Biosensors 2019, 9, 129, doi:10.3390/bios9040129 . . . . . . . . . . . . . .

Manon Giraud, François-Damien Delapierre, Anne Wijkhuisen, Pierre Bonville,

Mathieu Thévenin, Gregory Cannies, Marc Plaisance, Elodie Paul, Eric Ezan,

Stéphanie Simon, Claude Fermon, Cécile Féraudet-Tarisse and Guénaëlle Jasmin-Lebras

Evaluation of In-Flow Magnetoresistive Chip Cell—Counter as a Diagnostic Tool

Reprinted from: Biosensors 2019, 9, 105, doi:10.3390/bios9030105 . . . . . . . . . . . . .

Honeyeh Matbaechi Ettehad, Rahul Kumar Yadav, Subhajit Guha and Christian Wenger

Towards CMOS Integrated Microfluidics Using Dielectrophoretic Immobilization

Reprinted from: Biosensors 2019, 9, 77, doi:10.3390/bios9020077 . . . . . . . . . . . . 33

Vikram Surendran, Thomas Chiulli, Swetha Manoharan, Stephen Knisley,

Muthukumaran Packirisamy and Arvind Chandrasekaran

Acoustofluidic Micromixing Enabled Hybrid Integrated Colorimetric Sensing, for Rapid

Point-of-Care Measurement of Salivary Potassium

Reprinted from: Biosensors 2019, 9, 73, doi:10.3390/bios9020073

Patrick Risch, Dorothea Helmer, Frederik Kotz and Bastian E. Rapp

Analytical Solution of the Time-Dependent Microfluidic Poiseuille Flow in Rectangular Channel Cross-Sections and Its Numerical Implementation in Microsoft Excel

Reprinted from: Biosensors 2019, 9, 67, doi:10.3390/bios9020067

Zaidon T. Al-aqbi, Yiing C. Yap, Feng Li and Michael C. Breadmore

Integrated Microfluidic Devices Fabricated in Poly (Methyl Methacrylate) (PMMA) for On-site

Therapeutic Drug Monitoring of Aminoglycosides in Whole Blood

Reprinted from: Biosensors 2019, 9, 19, doi:10.3390/bios9010019

Shilun Feng, Elham Shirani and David W. Inglis

Droplets for Sampling and Transport of Chemical Signals in Biosensing: A Review

Reprinted from: Biosensors 2019, 9, 80, doi:10.3390/bios9020080 



\section{About the Editors}

David W. Inglis is Associate Professor in the School of Engineering at Macquarie University (Sydney Australia), where he is the leader of the Biomedical Microdevices Group. Dr. Inglis received his B.Sc. in Engineering Physics from the University of Alberta in 2001 and a Ph.D. in Electronic Materials and Devices from Princeton University in 2007. He is an expert in microfluidic particle separation and has published more than 40 journal articles which have been cited more than 2500 times in total.

Majid Ebrahimi Warkiani is Associate Professor in the School of Biomedical Engineering at UTS, Sydney, Australia. He received his Ph.D. in Mechanical Engineering from Nanyang Technological University (NTU) under the prestigious SINGA scholarship from A*STAR and undertook postdoctoral training at Massachusetts Institute of Technology (SMART Centre). He is an NHMRC-CD fellow and also a member of the Institute for Biomedical Materials \& Devices (IBMD) and Center for Health Technologies (CHT) at UTS.

Dr Warkiani's current research activities focus on three key areas of (i) microfluidics involving the design and development of novel microfluidic systems for particle and cell sorting (e.g., circulating tumor cells, fetal cells, and stem cells) for diagnostic and therapeutic applications; (ii) organ-on-a-chip involving the fabrication and characterization of novel 3D lab-on-a-chip systems (e.g., lung-on-a-chip, tumor-on-a-chip) to model physiological functions of tissues and organs; and (iii) 3D microprinting involving the design and development of novel miniaturized systems (e.g., micromixers, microcyclones) for basic and applied research.

Mohammad A. Qasaimeh is Assistant Professor of Mechanical and Biomedical Engineering at New York University Abu Dhabi (NYUAD), Abu Dhabi, UAE, and with the Mechanical and Aerospace Engineering Department at Tandon School of Engineering, New York University (NYU), New York, USA. He established the Advanced Microfluidics and Microdevices Laboratory (AMMLab) in 2014, and his current research interests include developing microfluidic and MEMS devices for clinical applications and point-of-care diagnostics. Recently, Dr. Qasaimeh was awarded the Technology Innovation Pioneers (TIP) Award during the TIP 2020 Summit. Prior to joining NYUAD, he was a Postdoctoral Research Associate at Massachusetts Institute of Technology and a Research Fellow at Harvard Medical School. Dr. Qasaimeh completed his Ph.D. degree in Biomedical Engineering at McGill University, where he received several prestigious fellowships and awards including the NSERC Postdoctoral Fellowship, the Alexander Graham Bell Graduate Scholarship, and the FQRNT Researchers Stars Award. Dr. Qasaimeh's research has been published in many peer-reviewed journals including Nature Communications, Advanced Biosystems, Lab on a Chip, iScience, Advanced Therapeutics, and Scientific Reports. He delivered more than 30 keynote and invited lectures at national and international conferences and is actively involved in organizing several local and international conferences. Currently, he is serving as a Co-Chair at the NYU Biomedical and Biosystems Conference series and as a Program Chair of the International Conference on Manipulation, Automation and Robotics at Small Scales (MARSS). Dr. Qasaimeh is serving as an Associate Editor with the IEEE Nanotechnology Magazine, a Topic Editor with the journal Biosensors, a Review Editor with the journal Frontiers in Bioengineering and Biotechnology, and an Editorial Board Member of Scientific Reports of the Nature Publishing Group. 
Weiqiang Chen is Associate Professor in the Departments of Mechanical and Aerospace Engineering and Biomedical Engineering at New York University. He is the recipient of the Biomedical Engineering Society Young Innovator Award of Cellular and Molecular Bioengineering (2019), the Chroma Young Investigator Award in Biomedical Engineering (2019), the Lab on a Chip Emerging Investigator Award (2018), the National Institute of Biomedical Imaging and Bioengineering Trailblazer Award (2018), the NYU Whitehead Fellowship in Biomedical and Biological Sciences (2017), the Goddard Junior Faculty Award (2017), the American Heart Association Scientist Development Award (2016), and the Baxter Young Investigator Award (2013). Dr. Chen's research interests are focused on lab-on-a-chip, biosensing, cell mechanobiology, stem cell biology, cancer biology, and immune engineering. 


\section{Preface to "Microfluidics for Biosensing and Diagnostics"}

We are pleased to present this Special Issue on sensing and diagnostics with microfluidics. Efforts to miniaturize sensing and diagnostic devices and to integrate multiple functions into one device have caused massive growth in the field of microfluidics and this integration is now recognized as an important feature of most new diagnostic approaches.

The field of microfluidics is exceptionally diverse. It attracts interest and contributions from physicists, chemists, and biologists as well as electrical, mechanical, chemical, and biomedical engineers. Working in such a diverse community poses many challenges arising from different training, different terminology, and different standards and expectations for data. This brief collection of papers highlights the spread of expertise that is involved in research aimed at developing biosensing and diagnostics using microfluidics.

David W. Inglis, Majid Ebrahimi Warkiani, Mohammad A. Qasaimeh, Weiqiang Chen Editors 

Article

\title{
Fast Active Merging of Microdroplets in Microfluidic Chambers Driven by Photo-Isomerisation of Azobenzene Based Surfactants
}

\author{
Zain Hayat ${ }^{1, \dagger}$, Nizar Bchellaoui ${ }^{1, \ddagger}$, Claire Deo ${ }^{2, \S}$, Rémi Métivier ${ }^{2}$, Nicolas Bogliotti ${ }^{2}$, Juan Xie ${ }^{2}$, \\ Malcolm Buckle ${ }^{3}$ and Abdel I. El Abed ${ }^{1, *}$ \\ 1 Laboratoire de Photonique Quantique et Moléculaire (LPQM), UMR 8537, Ecole Normale Supérieure Paris \\ Saclay, CentraleSupélec, CNRS, Université Paris-Saclay, 61 avenue du Président Wilson, 94235 Cachan, \\ France; ZAIN.HAYAT@ens-paris-saclay.fr (Z.H.); NIZAR.BCHELLAOUI@ens-paris-saclay.fr (N.B.) \\ 2 Photophysique et Photochimie Supramoléculaires et Macromoléculaires (PPSM), UMR 8531, Ecole Normale \\ Supérieure Paris Saclay, CNRS, Université Paris-Saclay, 61 avenue du Président Wilson, 94235 Cachan, \\ France; claire.deo@embl.de (C.D.); Remi.METIVIER@ppsm.ens-cachan.fr (R.M.); \\ NICOLAS.BOGLIOTTI@ens-paris-saclay.fr (N.B.); joanne.xie@ens-paris-saclay.fr (J.X.) \\ 3 Laboratoire de Biologie et Pharmacologie AppliquéE (LBPA), UMR 8113, Ecole Normale Supérieure Paris \\ Saclay, CNRS, Université Paris-Saclay, 61 avenue du Président Wilson, 94235 Cachan, France; \\ buckle@ens-paris-saclay.fr \\ * Correspondence: abdel.el-abed@ens-paris-saclay.fr \\ † Current address: Microsystèmes D'Analyse (MICA), Laboratoire D'analyse et D'architecture des Systèmes \\ (LAAS), CNRS, 31400 Toulouse, France. \\ $\ddagger$ Current address: Groupe D'étude de la Matière Condensée (GEMaC), Université de Versailles saint Quentin \\ en Yvelines, 78000 Versailles, France. \\ $\S$ Current address: Cell Biology and Biophysics Unit, European Molecular Biology Laboratory (EMBL), 69117 \\ Heidelberg, Germany.
}

Received: 7 October 2019; Accepted: 28 October 2019; Published: 1 November 2019

\begin{abstract}
In this work, we report on the development of a newly synthesized photoactive reversible azobenzene derived surfactant polymer, which enables active and fast control of the merging of microdroplets in microfluidic chambers, driven by a pulsed UV laser optical stimulus and the well known cis-trans photo-isomerisation of azobenzene groups. We show for the first time that merging of microdroplets can be achieved optically based on a photo-isomerization process with a high spatio-temporal resolution. Our results show that the physical process lying behind the merging of microdroplets is not driven by a change in surface activity of the droplet stabilizing surfactant under UV illumination (as originally expected), and they suggest an original mechanism for the merging of droplets based on the well-known opto-mechanical motion of azobenzene molecules triggered by light irradiation.
\end{abstract}

Keywords: microdroplets; photo-isomerisation; photokinetics; opto-mechanics; conformational states

\section{Introduction}

Many lab-on-a-chip (LoC) applications have become possible thanks to the ability to control mixing of different droplet contents, which enabled the sequencing of many complex bio-chemical and biological reactions with a high level of control and flexibility over the last decade; see for a review [1-7]. Hence, among all manipulation schemes allowed by droplet-based microfluidics technology [8-16], active merging of microdroplets (AMD) is probably one of the most important. It is generally achieved using a high alternating current (AC) voltage [17], or using a direct current (DC) voltage [18]. Nevertheless, light-driven merging of droplets is a more attractive approach since 
light provides not only high temporal and spatial resolutions but also wavelength and intensity tunability [19-21].

Recently, Dunkel et al. [22] showed that active merging of microdroplets can be achieved optically and very selectively using the photolysis process of photolabile surfactants [22]. In the present study, we consider a new strategy based on the photo-isomerization process (Figure 1) of a newly synthesized azobenzene derivative surfactant polymer, whose structure is given in Figure 2 and which is named in this study KryAz600, to achieve an active merging of water-in-oil (W/O) microdroplets using a picosecond (ps) pulsed UV laser.

This study was inspired initially by a previous work carried out by Takahashi et al. [23], who reported that a light-induced destabilization of an overall emulsion based on the photo-isomerization process of azobenzene-derived photosensitive surfactants, through the light induced interfacial activity change of gemini-like azobenzene derived surfactants and the conversion between a higher surface activity trans isomer and a lower surface activity cis isomer. This resulted in a destabilisation of the overall emulsion without the aim of achieving spatial differentiation and requesting several minutes of irradiation.

Our approach is different and is far from trivial. In fact, the dynamics of the change of azobenzene surfactants surface activity at the microscale, at which new interfaces are produced involve both the change of surface tension driven by the photo-isomerisation process and the diffusion of the new surfactant molecules to the interface, as well as the adsorption on the droplet surface, each partial step adding a typical time and length-scale [24]. Our approach is also different from the recent study reported by Dunkel et al. [22] as the mechanisms lying behind the two merging processes involved in the two studies are completely different. Moreover, photo-isomerization of azobenzene is fully reversible, which makes this new approach particularly suitable for the reuse of the photo-sensitive surfactant, which is generally produced in a small quantity.

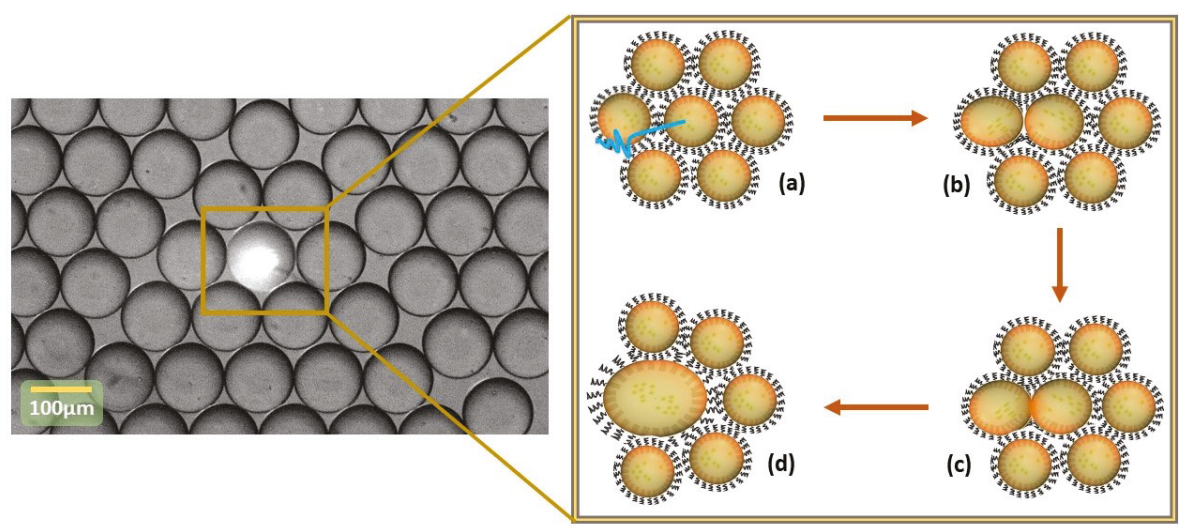

Figure 1. Light-driven merging process principle. (a) Targeting droplets and change of surface activity of the surfactant molecules at the droplet surface under laser irradiation, (b) depletion of the surfactant molecules at the the droplet interface, (c) merging induced following UV laser irradiation and the trans to cis photo-conversion, (d) targeted droplets after merging. 

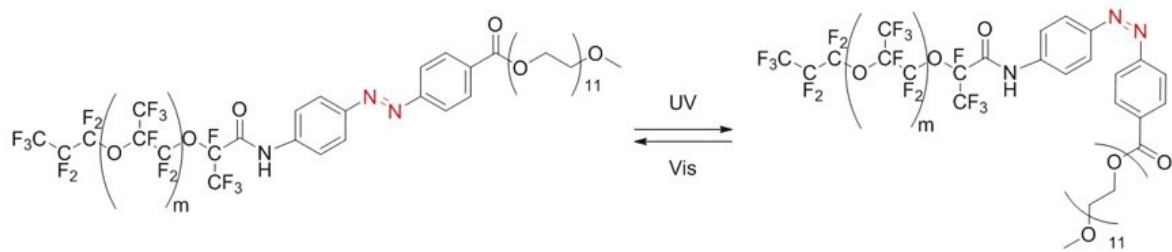

Figure 2. Stable trans form (left) and metastable cis form (right) of the synthesized KryAz600 molecule; trans to cis transition occurs under UV irradiation and back to trans occurs under visible light.

\section{Materials and Methods}

\subsection{Chemicals}

Azobenzene derived molecules possess two stable geometric isomers: an energetically stable trans form and a meta-stable cis form. For most azobenzenes, molecules can be optically isomerized from trans to cis using light in the near UV and Visible: upon absorption of a photon with a wavelength in the near UV (around $330 \mathrm{~nm}$ ), molecules convert, with high efficiency, from the trans isomer into the cis isomer. A second photon with a wavelength in the visible range (around $440 \mathrm{~nm}$ ) can induce the back-conversion. UV illumination can also enable conversion of azobenzene molecules from a cis form to a trans form as photons of UV light have higher energy than visible light, which is sufficient to induce cis to trans isomerization. Azobenzene photo-isomerization is completely reversible and both forward and reverse photoisomerizations typically exhibit picosecond timescales. The trans isomer is thermodynamically more stable than the cis isomer, by approximately $50-100 \mathrm{~kJ} / \mathrm{mol}$ and the energy barrier for thermal isomerization is in the order of $100-150 \mathrm{~kJ} / \mathrm{mol}$. Hence, in the dark, the cis isomer thermally relaxes back to the trans isomer on a timescale ranging from milliseconds to hours, or even days, depending on the substitution pattern around the azobenzene group and the local environment of the molecules.

We synthesized a new fluorinated azobenzene derivative surfactant polymer, named in this study KryAz600. It consists of a triblock copolymer surfactant, composed of a perfluoro-polyether (PFPE) hydrophobic chain, linked to a polyethylene-glycol (PEG-600) hydrophilic chain (Sigma-Aldrich, Saint-Quentin Fallavier, France) through an azobenzene group, as shown in Figure 2. The PFPE hydrophobic chain was derived from a commercially available carboxy-terminated fluorinated polymer, namely Krytox 157-FSH (Dupont) and linked to the azobenzene group following a similar procedure as described in detail by Lee et al. [25], see also supporting information at (S1) for details.

\subsection{Experimental Setup}

Nemesys syringe pumps (Cetoni $\mathrm{GmbH}$, Korbussen, Germany) were used to fabricate monodisperse drops of size range $50 \mu \mathrm{m}$ to $150 \mu \mathrm{m}$ depending upon the need. To study and sort droplets, the optics part consists of a ps-pulsed UV laser source, with a peak wavelength at $355 \mathrm{~nm}$ and delivering $15.4 \mu \mathrm{J}$ of energy per pulse. In order to make the laser spot size adequate, the laser beam was cleaned and band-limited for enhanced detection ( $20 \mathrm{~nm}$ filter F). Figure 3 illustrates the optical path for the laser source. After the filter, the source was staged up and reflected by dichroic mirror (DM) (Tx = $506 \mathrm{~nm}$, Semrock, Rochester, NY, USA) to the sample where a microscope objective $(2 \times, 4 \times$, or $10 \times$ Olympus Inverted Microscope) targets and acquires the reflected/scattered signal from the droplet under observation. At the detection side we used a standard speed camera for recording and visual inspection of the droplet generation, manipulation, and merging. For detection, a photo-multiplier tube (Hamamatsu) was used to monitor droplet generation frequency. For optimized $100 \mu \mathrm{m}$ drop-size $(500 \mu \mathrm{L} / \mathrm{h}$ and $100 \mu \mathrm{L} / \mathrm{h}$ for the continuous and dispersed phase), the droplet 
frequency was found to be about 250 drops/second (scheme for droplet frequency was derived from the method discussed in [15]).

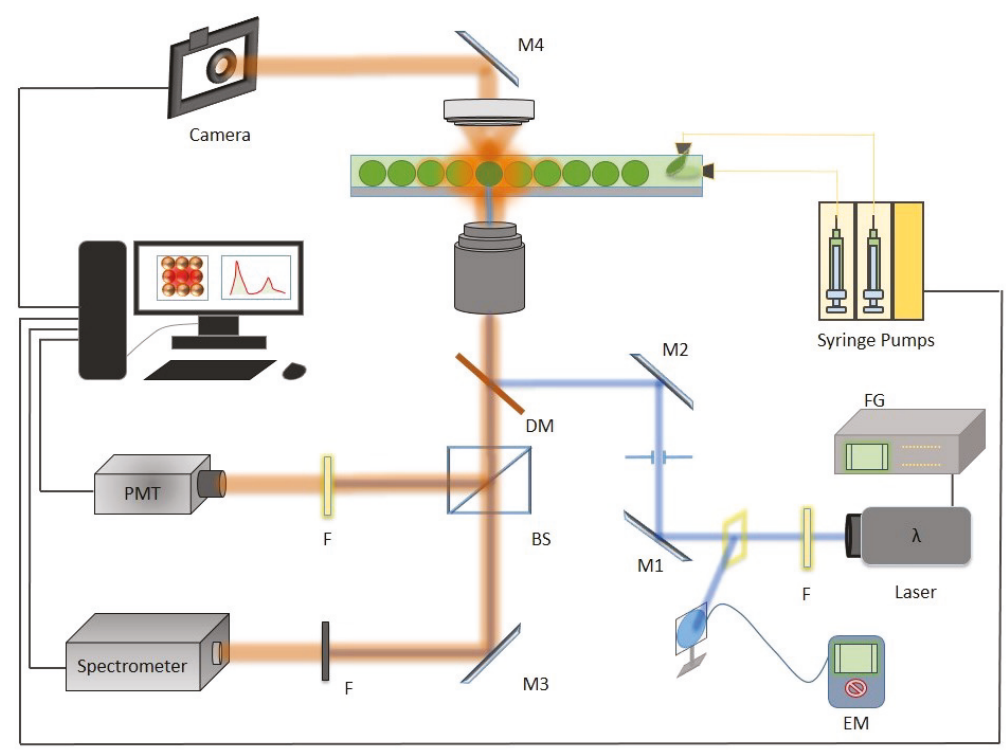

Figure 3. The setup containing the droplet generation assembly, optics and detection unit. On the optical path, the ps-pulsed source (355 nm), F (filter), M1 and M2 mirrors for step-up, dichoric mirror (DM) $(506 \mathrm{~nm})$, camera with notch filter, BS (beam splitter) to reflect the acquired signal to detector (setup adapted from [15]).

\subsection{Design and Microfabrication}

Droplets were generated in a flow-focusing microfluidic device, which was fabricated using the standard soft-lithography technique, employing PDMS (Polydimethylsiloxane) for replica molding. The constructed microfluidic device had a square drive channel (guide length after the drop-maker) and a rectangular micro-analysis chamber constructed by stepper lithographic pattern. The advantage of the dual stage chamber is to facilitate the droplet in maintaining a spherical shape, which in turn reduced the pressure coalescence of microdroplets. Droplet stability can be increased by providing extra time to the surfactant molecule in order to localize around the droplet (exterior) wall. This attribute was achieved by increasing the drive channel length to almost three times the length of the drive channel mentioned in previous work [22].

The drive channel length was set to about $2100 \mu \mathrm{m}$ (Figure 4a), which is long enough to allow the oil soluble surfactant molecules to build a stabilizing monolayer around the droplets before the droplet come in contact with each other at the entrance, where they may merge. After the drive channel, droplets enter a rectangular chamber of the dimensions $4000 \times 1200 \mu \mathrm{m}$ (Figure 4b) acting as droplet storage and an analysis chamber. At the end of the device assembly, a zig-zag channel leads to output for the droplet collection (Figure 4c). The advantage of droplet collection is to utilize the reversibility of the photo-active reversible compound and to perform off-chip micro-particle studies. 


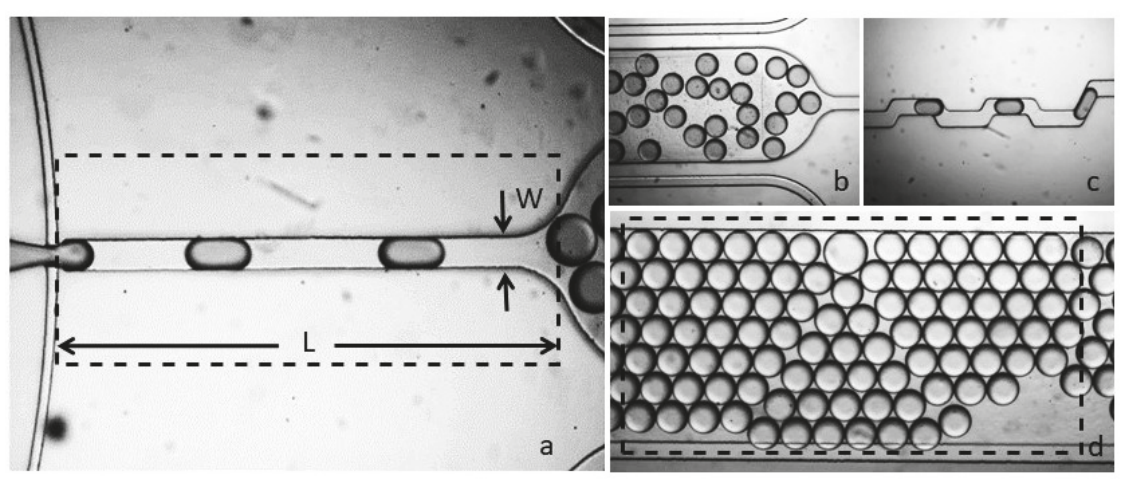

Figure 4. The fabricated flow-focusing assembly, (a) drive channel with width $\mathrm{W}$ and length $\mathrm{L}$, (b) droplet generation at $500 \mathrm{uL} / \mathrm{h}$ and $100 \mathrm{uL} / \mathrm{h}$ for drive and dispersed phase, (c) output channel for droplet frequency monitoring, (d) micro-analysis and storage chamber.

In order to make the device interior compatible with the oil-phase, the interior walls of the complete assembly was functionalized by commercially available surface coating agent, which consisted of a $2 \%$ solution of perfluoroctyl-dimethylsiloxane dissolved in HFE7100 fluorocarbon oil (3M). This coating enhanced the wettability of the channel walls and also reduced the risk of diffusion of the surfactant molecules to the PDMS. For the droplet generation, we used a fluorinated oil phase (HFE 7500, 3M, density $1.62 \mathrm{~g} / \mathrm{cm}^{3}$ ).

Photochromic reactions were induced in-situ by a continuous irradiation $\mathrm{Hg} / \mathrm{Xe}$ lamp (Hamamatsu, LC6 Lightningcure, $200 \mathrm{~W}$ ) equipped with narrow band interference filters of appropriate wavelengths. The irradiation power was measured using a photodiode from Ophir (PD300-UV). The photochromic quantum yields were determined by probing the sample with a Xenon lamp during the course of the light irradiation. Absorption changes were monitored by a charge coupled device (CCD) camera mounted on a spectrometer (Ocean Optics, Largo, FL, USA).

\section{Results and Discussion}

\subsection{KryAz600 Surfactant Photokinetics}

Likewise, most azobenzene derivatives, KryAz600 molecules switch under UV illumination from a stable trans form (t-KryAz600) to a metastable cis form (c-KryAz600). The trans form is characterized by a large absorption band with a maximum absorption around $335 \mathrm{~nm}$ (in HFE 7500 oil), while c-KryAz600 form has a weaker absorption band with a maximum absorption around $440 \mathrm{~nm}$, as shown in Figure 5.

Our results show that KryAz600 molecules transit spontaneously in the dark from the cis form to the trans form with a constant time as small as $10^{-5} / \mathrm{s}$. This value was deduced from the exponential fit of the absorption curve of c-KryAz600 at $330 \mathrm{~nm}$ versus time, as shown in Figure 5. It corresponds to a half-life $t_{1 / 2} \simeq 18 \mathrm{~h}$. It is worth noting that the relatively long half-life of KryAz600 molecules is of great importance in our study since the cis form relaxes back very slowly to the trans form, if no UV illumination is used. 

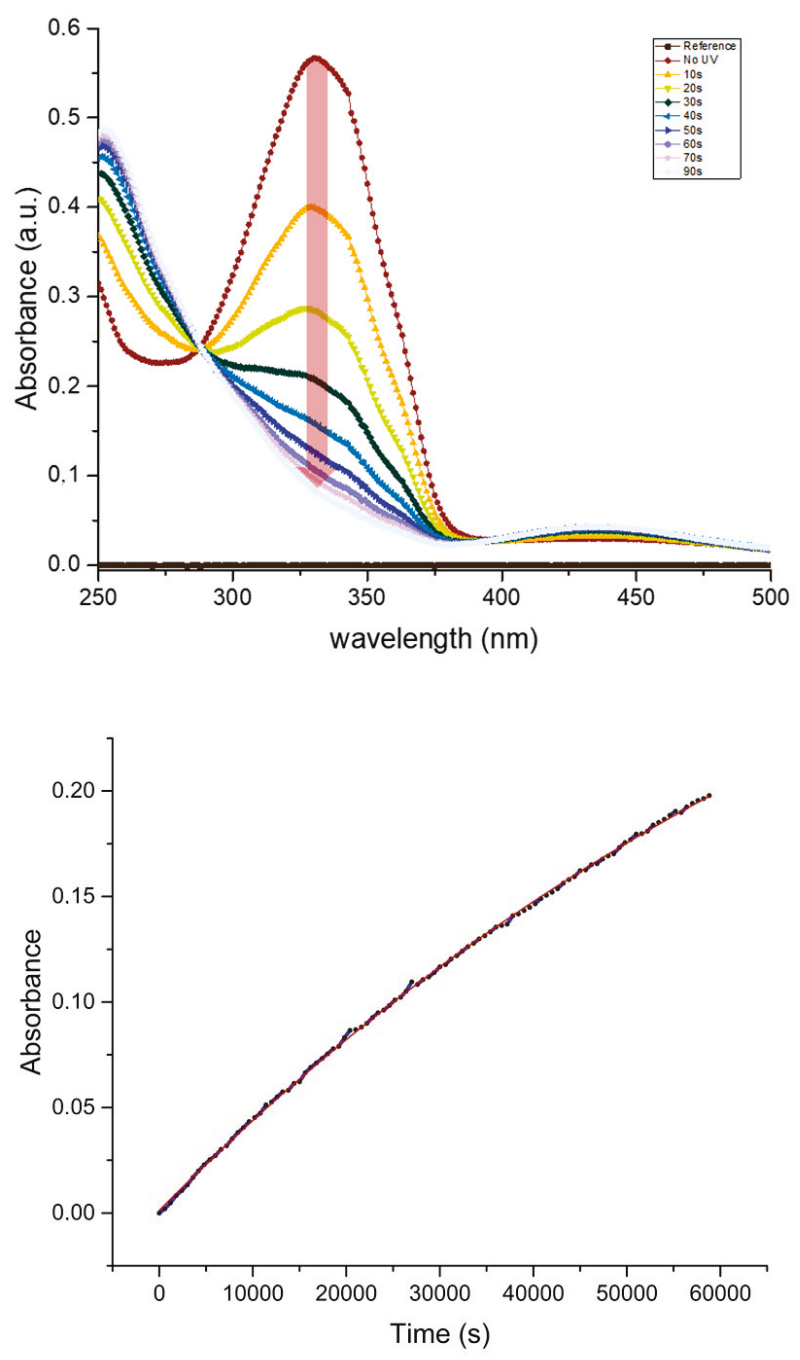

Figure 5. (Upper) Conversion of the surfactant state under UV illumination, Top curve (red) being the first measurement with no UV treatment and subsequent state change transition of surfactant by $10 \mathrm{~s}$ of exposure to a UV lamp with power of $6.7 \mathrm{~mW}$ at $365 \mathrm{~nm}$ wavelength; (Bottom) slow exponential rise of the $335 \mathrm{~nm}$ absorption band intensity of the t-KryAz600 form in dark, this corresponds to a half-life $t_{1 / 2} \simeq 18 \mathrm{~h}$ of the c-KryAz600 $\rightarrow \mathrm{t}-\mathrm{KryAz} 600$ transition.

\subsection{Microdroplets Stability Versus Surfactant Conformation and Concentration}

Monodisperse microdroplets with a size of about $100 \mu \mathrm{m}$ were prepared using different surfactant concentrations dissolved in HFE7500 oil, ranging from $1 \mathrm{mM}$ down to $15 \mu \mathrm{M}$.

In order to quantify the effect of the cis and trans conformations on the stability of droplets, a control experiment was first conducted. Two samples of equal concentration of $1 \mathrm{mM}\left(C_{0}\right)$ were prepared, one was left in the dark (overnight) in order to allow for all surfactant molecules present in 
the solution to transit to the thermodynamically stable trans state, and the other sample was illuminated with a $235 \mathrm{~nm}$ UV lamp for $1 \mathrm{~h}$.

Droplets that were prepared using the non irradiated surfactant solution exhibited long term stability (Figure 6a) where as the droplets prepared using UV illuminated surfactant solution merged immediately in the observation chamber (Figure 6b). This shows that cis conformation of KryAz600 molecules is not suitable to ensure droplet stabilization. A quick glance at the molecule structure of Figure 2 shows that the cis conformation is less suitable to achieve a close packing of surfactant molecules at the droplet interface, than could be done using the trans isomer. In other words, cis isomer molecules lead to a lower surface density of surfactant molecules around the droplet and hence to a higher interfacial tension.

It is worth noting, that UV illuminated surfactant solution, when left in the dark overnight, enables us again to produce stable droplets. This demonstrates the reversibility of the process and the possibility to reuse surfactant solution for further experiments. Our observations show also that the merging of microdroplets under laser illumination is not the result of the thermo-capillary effect of the laser beam, since thermal effects are present for both droplets, prepared with the two types of isomers.
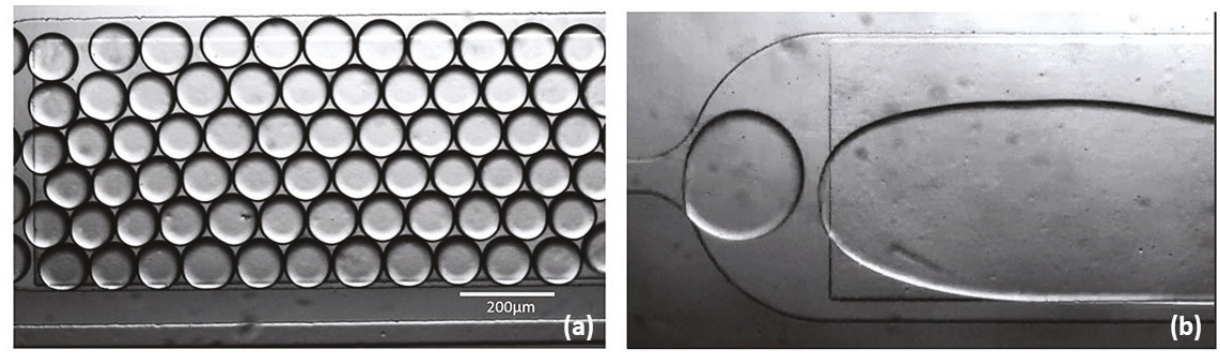

Figure 6. Surfactant KryAz600, (a) mono-disperse (water-in-oil) droplets of stable trans state, (b) effect of UV exposure, unstable cis state causing coalescence of micro-droplets.

Also, because at the micrometer scale, the diffusion of surfactant molecules cannot be neglected, we found it necessary to investigate the relation between the merging time and the concentration of the surfactant molecules in the carrier fluorocarbon oil. This is particularly important in our study since a focused pulsed laser with a micrometer sized footprint is used for photo-isomerization.

Water-in-oil (W/O)droplets were produced and collected in an on-chip micro-analysis chamber with different surfactant concentrations, decreasing from $1 \mathrm{mM}\left(C_{0}\right)$ to $15.6 \mu \mathrm{M}$. This process depends on the kinetics of the depletion of surfactants under laser illumination. The first concentration, $C_{0}=1 \mathrm{mM}$ of the surfactant, was found to lead to very stable microdroplets with no merging process with irradiation times smaller than $10 \mathrm{~min}$, whereas a fast merging $(\sim 1 \mathrm{~s})$ could be achieved with a concentration value of $25 \mu \mathrm{M}$. It is worth noting that for smaller concentration values of surfactant $(c<25 \mu \mathrm{M})$, a spontaneous merging of droplets is observed, which indicates that there are not enough surfactant molecules in the carrier oil solution for droplets stabilization. Successive dilutions of the surfactant solution resulted in a decrease of the merging time from about $10 \mathrm{~min}$ for $C_{0}(1 \mathrm{mM})$ to $1 \mathrm{~s}$ for $25 \mu \mathrm{M}$. For lower concentrations, droplets were observed to be highly unstable. These results clearly indicate a decrease in merging time as the concentration of surfactant molecules decreases. Merging reduced sufficiently from almost $6 \mathrm{~s}$ for $62.5 \mu \mathrm{M}$ to $1 \mathrm{~s}$ for $15.6 \mu \mathrm{M}$. This result may be interpreted as follows. As the concentration reduces, the number of idle-molecules in bulk drive phase take more time to replace the targeted molecules. Also, since the surfactant molecules are not oriented in a specific order, so the lower the concentration, the higher the diffusion time for the idle molecules to reach the depleted area.

To better understand these results, let us first calculate the flow time, $t_{\text {flow }}$, that each droplet takes to reach the observation micorchamber; $t_{\text {flow }}$ corresponds to the flight time of droplets along the 
drive channel, from the nozzle to the end of the main channel, above which droplets start to collide with each other. Considering the length $L=2100 \mu \mathrm{m}$ of the drive channel and the mean velocity of microdroplets in this region, $v \simeq 30 \mathrm{~mm} / \mathrm{s}$, one finds:

$$
t_{\text {flow }}=\frac{L}{v_{\text {flow }}} \simeq 70 \mathrm{~ms} .
$$

To ensure droplet stability, $t_{\text {flow }}$ should be greater than the diffusion time, $t_{\text {dif }}$, which is approximately the necessary time lapse for building a stabilizing surfactant layer around the droplets to prevent their coalescence. Indeed, during $t_{\text {flow }}$, a given number of surfactant molecules diffuse from the bulk phase and adsorb arround the flowing droplet interface to form a stabilizing surfactant layer. Considering the total area $S_{\text {drop }}=4 \pi R^{2}$ of a microdroplet with a radius $R(\simeq 50 \mu \mathrm{m})$, the maximum packing of surfactant molecules, with a typical lateral dimension $\delta \sim 2 \mathrm{~nm}$, i.e., with a molecular area $A \sim \delta^{2}$, at the interface of the droplet is achieved with $n$ molecules when $4 \pi R^{2} \sim n \delta^{2}$. Droplets will be stable when the number of molecules at the interface is larger than a fraction $0<f<1$ of the maximum packing; therefore, the stability condition reads

$$
n \geq \frac{4 f \pi R^{2}}{A}
$$

According to Baret et al., [26] microdroplets become stable when the number of molecules at the interface becomes larger than a fraction $f \sim 0.1$ of the maximum packing; therefore, the stability condition for microdroplets of radius $R$ can be expressed as follows:

$$
n \sim \frac{0.4 \pi R^{2}}{A} \text { molecules. }
$$

The number of free surfactant molecules required for the stabilization of the droplet interface, $n$, can be assumed to be dispersed in a volume $V_{\epsilon}=4 \pi R^{2} \epsilon$ surrounding the droplet over a distance $\epsilon$ from the interface $(\epsilon<<R)$. Hence, $n$ can be estimated as $n \sim 4 \pi R^{2} \epsilon c N_{A}$, where $N_{A}$ is Avogadro's number. In a diffusion limited process, surfactant molecules confined in the volume $V_{\epsilon}$ will reach the droplet interface within a time $t_{\text {dif }}$ given by the diffusion law: $\epsilon^{2} \sim D \times t_{\text {dif }}$. It is possible thus to estimate an experimental value for the diffusion time $t_{\text {dif }}$ according to the following equation:

$$
t_{\text {dif }} \sim \frac{0.01}{\left(N_{A} c A\right)^{2} D} .
$$

Hence, for $c=25 \mu \mathrm{M}$, one finds $t_{\text {dif }} \sim 30 \mathrm{~ms}\left(<t_{\text {flow }}\right)$, whereas for $c=15 \mu \mathrm{M}$, one finds $t_{\text {dif }} \sim 70 \mathrm{~ms}$ $\left(\simeq t_{\text {flow }}\right)$. These results are in good agreement with the observed surfactant concentration threshold for droplet stability, which was found to lay in the $25-15 \mu \mathrm{M}$ range.

To further analyze the merging behavior of microdroplets under irradiation, different sweeps of the laser beam over the whole micro-chamber were performed and first and second merging of the one drop to its neighbor and later (second merging) with the two times big merged drop to its neighboring droplet. Results presented in Figure 7 show a non-uniform but correlated first and second merging times. The rationale behind two merging observations relates to the change in volume and a slight increase in the merging time. Consider Figure 7, on left, two drops selected for merging and on right the big merged drop. For two separate drops of $100 \mu \mathrm{m}$, the calculated volume was $0.5 \mathrm{~nL}$ each, while after merging the the big drop had a volume of $4 \mathrm{~nL}$, thus eight times increase in volume. From the classical inverse relation of concentration with volume, the overall change in concentration of big merged drop was 0.125 times the concentration of the drive phase. Upon targeted merging of that droplet with the usual droplet caused a slight increase in merging time, depicted as a green curve in Figure 7. 

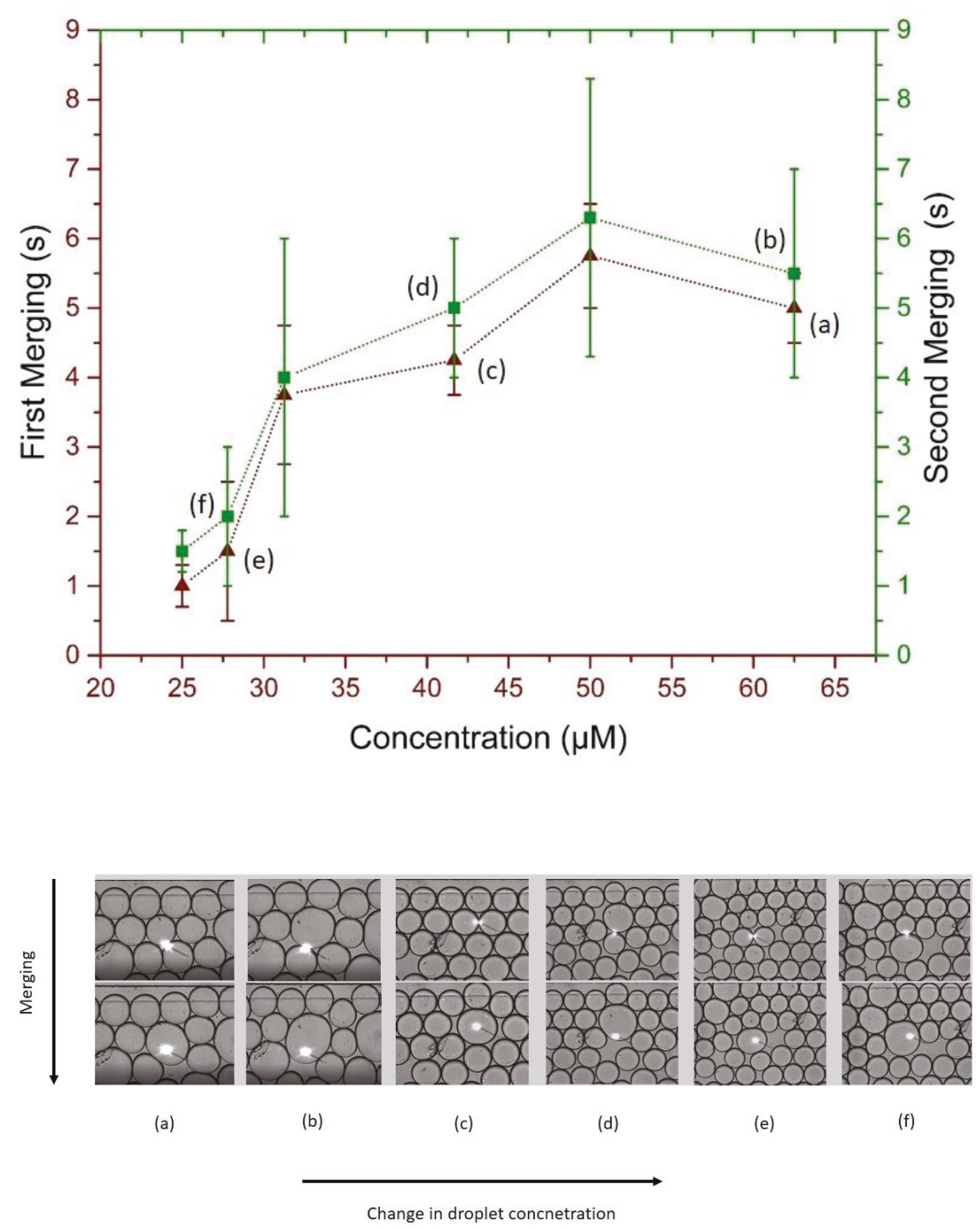

Figure 7. (Upper) Merging time scale of irradiated droplets, maroon (triangles) for first merging and green (squares) for second merging, (Bottom) optical micro-graphs of the first and second merging and resulting change in targeted droplet volume.

\subsection{Suggested Opto-Mechanical Model for Droplet Merging Mechanism}

Unlike in the work reported by Takahashi et al. [23], where a broad UV illumination during several minutes of the overall emulsion resulted in a destabilisation of the overall emulsion, photo-isomerization is achieved in our study using a ps UV laser which is focused on a very tiny fraction of the emulsion. It lasts only a few seconds before droplet merging is achieved. At such time and space scales, the change in the activity of surfactant molecules involves both a change of surface tension driven by the photo-isomerisation process and the diffusion of new surfactant molecules from 
the bulk solution to the interface, as well as the adsorption on the droplet surface. If one considers a diffusion limited merging process mechanism, to achieve droplet interface destabilization and merging, the rate of photoisomerized surfactant molecules under pulsed UV laser illumination should be higher than the diffusion rate of non irradiated surfactant molecules from the bulk solution to the target droplet interface. Nevertheless, in the particular case of photo-isomerization, one should take account of the fact that the higher energy UV illumination can induce not only isomerization from trans to cis form but it can also induce the reverse transformation on cis molecules present under the UV light spot. Hence, when a pulsed UV laser is used, if one illuminates locally, for instance, a population of A isomers during a first pulse to $B$ isomers, then the transformed B isomers would go back to their initial conformation (A) under illumination during the following pulse, unless diffusion is fast enough to deplete the illuminated region of $B$ isomers during the first pulse of the UV laser.

Though UV light can drive both cis to trans and trans to cis photo-isomerizations, one should emphasize that a longer term UV illumination of azobenzene molecules would not lead in average to a mixture of 50\% of molecules in each state. Indeed, the forward (trans to cis) and backward (cis to trans) photo-reactions of azobenzene molecules do not show the same quantum yield, i.e., the probability of inducing the photo-reaction after absorption of a photon. Therefore, the ratio of trans and cis isomers upon long term UV irradiation can be much higher than $50 \%$. In the present case, this ratio is higher than $85 \%$ under $365 \mathrm{~nm}$ illumination as can be deduced from the absorbance curves of KryAz600 molecules around $335 \mathrm{~nm}$ after $90 \mathrm{~s}$ of illumination vs. their absorbance before irradiation $(0 \mathrm{~s})$, as shown in Figure 5.

Let us calculate the diffusion distance $\epsilon$ of surfactant molecules in the oil solution during the time lapse $\tau_{\text {pulse }}$ of a first single pulse, which is equal to $1 \mathrm{~ms}$ in our experiments (and which should not be confused with the duration of a pulse $\simeq 400 \mathrm{ps}$ ). Using diffusion law, and considering a typical value of the diffusion coefficient of surfactant molecules in HFE7500 oil, about $D \simeq 10^{-10} \mathrm{~m}^{2} / \mathrm{s}[26,27]$, one finds a mean diffusion distance of

$$
\epsilon \sim \sqrt{D \tau_{\text {pulse }}} \sim 0.3 \mu \mathrm{m},
$$

During the time lapse of one pulse. Even if considering a diffusion coefficient which is two orders of magnitude higher than the previous one, as reported by Dunkel et al. [22], one would find a mean diffusion distance of about $\epsilon \sim 3 \mu \mathrm{m}$ during each pulse. It is interesting to note that such values are much smaller than the size of the illuminated area with the focused laser beam, which is, $\sim 30 \mu \mathrm{m}$ in our experiments. This rough estimation of the diffusion of surfactant molecules during the time lapse between two successive illuminations shows that a large proportion of isomerized molecules during the first pulse will be still present in the illuminated area when the following light pulse triggers and hence cis isomers will recover their initial conformation (trans) under this second UV laser pulse.

Consequently, one should conclude that the observed merging process of droplets cannot be attributed to a change of surface activity of surfactant molecules under illumination, as surfactant molecules switch continuously from trans to cis and from cis to trans under successive UV laser pulses. The merging process should be based on opto-mechanical effects due to the well-known change of the length of the cis and trans azobenzene groups under illumination. Photo-isomerization reduces the distance between the 4 and $4^{\prime}$ positions of azobenzene groups from $0.99 \mathrm{~nm}$ in the trans state to $0.55 \mathrm{~nm}$ for the cis state [28]. Consequently, UV laser illumination produces geometric changes in the azobenzene group, which should translate into larger-scale motions extending to the overall surfactant droplet interface layer. After approximately one second of illumination, the oscillation of the droplet interface should lead to the merging of the droplets.

\section{Conclusions}

Active merging of microdroplets is one of the most important manipulations enabled by droplet-microfluidics, as it enables for on demand droplet targeting and sequencing of complex 
chemical and biological reactions. Nevertheless, the standard voltage-based methods are generally limited to spatially isolated pairs of droplets, as the long-range effect of the applied electric field does not have the potential to differentiate between flowing or confined droplets. We developed an optofluidic approach, which overcomes such limitations and allows for a rapid controlled coalescence of targeted microdroplets thanks to the application of a ps UV pulsed laser. Our approach is based on the photo-isomerization process of an azobenzene-derived surfactant. The success of such controlled coalescence is far from trivial. In fact, the dynamics of the change of surface tension at the microscale driven by photo-isomerisation processes; the diffusion of the new surfactant molecules to the interface; as well as the adsorption on the droplet surface, are complex and not well understood. By incorporating light activated switching in the surfactant, release or mixing of droplets contents could be programmed. In this study, we present the fast and dynamic switching of azobenzene derived surfactant for controlled coalescence of spatially selectable microdroplets as proof of concept.

Supplementary Materials: The following are available online at http:/ /www.mdpi.com/2079-6374/9/4/129/s1, Figure S1: Chemical synthesis procedure of KryAz600 surfactant molecules.

Author Contributions: Conceptualization, M.B. and A.I.E.A.; Data curation, Z.H., N.B. (Nizar Bchellaoui), R.M. and A.I.E.A.; Formal analysis, Z.H. and A.I.E.A.; Funding acquisition, M.B. and A.I.E.A.; Investigation, Z.H., R.M. and A.I.E.A.; Methodology, C.D., Juan Xie, M.B. and A.I.E.A.; Project administration, M.B. and A.I.E.A.; Resources, C.D., Remi Metivier, N.B. (Nicolas Bogliotti), J.X., M.B. and A.I.E.A.; Software, Z.H. and A.I.E.A.; Supervision, N.B. (Nicolas Bogliotti), M.B. and A.I.E.A.; Validation, Z.H. and A.I.E.A.; Visualization, Z.H. and A.I.E.A.; Writing-original draft, Z.H. and A.I.E.A.; Writing-review editing, A.I.E.A.

Funding: This research received no external funding.

Acknowledgments: We thank Emile Auria for his contribution to the synthesis of the azobenzene-derived surfactant.

Conflicts of Interest: The authors declare no conflict of interests.

\section{References}

1. Gu, H.; Duits, M.H.G.; Mugele, F. Droplets Formation and Merging in Two-Phase Flow Microfluidics. Int. J. Mol. Sci. 2011, 12, 2572-2597. [CrossRef] [PubMed]

2. Agresti, J.J.; Antipov, E.; Abate, A.R.; Ahn, K.; Rowat, A.C.; Baret, J.C.; Marquez, M.; Klibanov, A.M.; Griffiths, A.D.; Weitz, D.A. Ultra-high-throughput screening in drop-based microfluidics for directed evolution. Proc. Natl. Acad. Sci. USA 2010, 107, 4004-4009. [CrossRef] [PubMed]

3. Taly, V.; Pekin, D.; Abed, A.E.; Laurent-Puig, P. Detecting biomarkers with microdroplet technology. Trends Mol. Med. 2012, 18, 405-416. [CrossRef] [PubMed]

4. deMello, A.J. Control and detection of chemical reactions in microfluidic systems. Nature 2006, 442, 394-402. doi:10.1038/nature05062. [CrossRef] [PubMed]

5. Song, H.; Chen, D.L.; Ismagilov, R.F. Reactions in Droplets in Microfluidic Channels. Angew. Chem. Int. Ed. 2006, 45, 7336-7356. [CrossRef]

6. Witters, D.; Sun, B.; Begolo, S.; Rodriguez-Manzano, J.; Robles, W.; Ismagilov, R.F. Digital biology and chemistry. Lab Chip 2014, 14, 3225-3232. [CrossRef]

7. Kumacheva, E.; Garstecki, P. Microfluidic Reactors for Polymer Particles; Wiley: Chichester, UK, 2011.

8. Thorsen, T.; Robert, R.W.; Arnold, F.H.; Quake, S.R. Dynamic Pattern Formation in a Vesicle-Generating Microfluidic Device. Phys. Rev. Lett. 2001, 86, 4163-4166. [CrossRef]

9. Thorsen, T.; Maerkl, S.J.; Quake, S.R. Microfluidic Large Scale Integration. Science 2002, 298, 580-584. [CrossRef]

10. Teh, S.Y.; Lin, R.; Hung, L.H.; Lee, A.P. Droplet microfluidics. Lab Chip 2008, 8, 198-220. [CrossRef]

11. Guo, M.T.; Rotem, A.; Heyman, J.A.; Weitz, D.A. Droplet microfluidics for high-throughput biological assays. Lab Chip 2012, 12, 2146-2155. [CrossRef]

12. Dak, P.; Ebrahimi, A.; Swaminathan, V.; Duarte-Guevara, C.; Bashir, R.; Alam, M. Droplet-based biosensing for lab-on-a-chip, open microfluidics platforms. Biosensors 2016, 6, 14. [CrossRef] [PubMed]

13. Wang, J.; Li, Y.; Wang, X.; Wang, J.; Tian, H.; Zhao, P.; Tian, Y.; Gu, Y.; Wang, L.; Wang, C. Droplet microfluidics for the production of microparticles and nanoparticles. Micromachines 2017, 8, 22. [CrossRef] 
14. Bchellaoui, N.; Hayat, Z.; Mami, M.; Dorbez-Sridi, R.; El Abed, A.I. Microfluidic-assisted formation of highly monodisperse and mesoporous silica soft microcapsules. Sci. Rep. 2017, 7, 16326. [CrossRef]

15. Hayat, Z.; El Abed, A. High-Throughput Optofluidic Acquisition of Microdroplets in Microfluidic Systems. Micromachines 2018, 9, 183. [CrossRef] [PubMed]

16. Sánchez Barea, J.; Lee, J.; Kang, D.K. Recent Advances in Droplet-based Microfluidic Technologies for Biochemistry and Molecular Biology. Micromachines 2019, 10, 412. [CrossRef]

17. Chabert, M.; Dorfman, K.; Viovy, J.L. Droplet fusion by alternating current (AC) field electro-coalescence in microchannels. Electrophoresis 2005, 26, 3706-3715. [CrossRef]

18. Link, D.R.; Grasland-Mongrain, E.; Duri, A.; Sarrazin, F.; Cheng, Z.; Cristobal, G.; Marquez, M.; Weitz, D.A. Electric control of droplets in microfluidic devices. Angew. Chem. Int. Ed. 2006, 45, 2556-2560. [CrossRef]

19. Mayer, G.; Heckel, A. Biologically Active Molecules with a "Light Switch". Angew. Chem. Int. Ed. 2006, 45, 4900-4921. [CrossRef]

20. Gorostiza, P.; Isacoff, E.Y. Optical Switches for Remote and Noninvasive Control of Cell Signaling. Science 2008, 322, 395-399. [CrossRef]

21. Baigl, D. Photo-actuation of liquids for light-driven microfluidics: state of the art and perspectives. Lab Chip 2012, 12, 3637-3653. [CrossRef]

22. Dunkel, P.; Hayat, Z.; Barosi, A.; Bchellaoui, N.; Dhimane, H.; Dalko, P.I.; El Abed, A.I. Photolysis-driven merging of microdroplets in microfluidic chambers. Lab Chip 2016, 16, 1484-1491. [CrossRef] [PubMed]

23. Takahashi, Y.; Fukuyasu, K.; Horiuchi, T.; Kondo, Y.; Stroeve, P. Photoinduced Demulsification of Emulsions Using a Photoresponsive Gemini Surfactant. Langmuir 2014, 30, 41-47. [CrossRef] [PubMed]

24. Brosseau, Q.; Vrignon, J.; Baret, J.C. Microfluidic Dynamic Interfacial Tensiometry ([small mu ]DIT). Soft Matter 2014, 10, 3066-3076. [CrossRef] [PubMed]

25. Lee, M.; Collins, J.W.; Aubrecht, D.M.; Sperling, R.A.; Solomon, L.; Ha, J.W.; Yi, G.R.; Weitz, D.A.; Manoharan, V.N. Synchronized reinjection and coalescence of droplets in microfluidics. Lab Chip 2014, 14, 509-513. [CrossRef]

26. Baret, J.C.; Kleinschmidt, F.; Harrak, A.E.; Griffiths, A.D. Kinetic Aspects of Emulsion Stabilization by Surfactants: A Microfluidic Analysis. Langmuir 2009, 25, 6088-6093. [CrossRef]

27. Rosen, M.J. Surfactants and Interfacial Phenomena, 3rd ed.; Wiley: Hoboken, NJ, USA, 2004.

28. Mahimwalla, Z.; Yager, K.G.; Mamiya, J.I.; Shishido, A.; Priimagi, A.; Barrett, C.J. Azobenzene photomechanics: prospects and potential applications. Polym. Bull. 2012, 69, 967-1006. [CrossRef]

(C) 2019 by the authors. Licensee MDPI, Basel, Switzerland. This article is an open access article distributed under the terms and conditions of the Creative Commons Attribution (CC BY) license (http://creativecommons.org/licenses/by/4.0/). 


\title{
Article \\ Evaluation of In-Flow Magnetoresistive Chip Cell-Counter as a Diagnostic Tool
}

\author{
Manon Giraud ${ }^{1,2}$, François-Damien Delapierre ${ }^{1}$, Anne Wijkhuisen ${ }^{2}$, Pierre Bonville ${ }^{1}$, \\ Mathieu Thévenin ${ }^{1}$, Gregory Cannies ${ }^{1}$, Marc Plaisance ${ }^{2}$, Elodie Paul ${ }^{1}$, Eric Ezan ${ }^{3}$, \\ Stéphanie Simon ${ }^{2}$, Claude Fermon ${ }^{1}$, Cécile Féraudet-Tarisse ${ }^{2,+}$ and \\ Guénaëlle Jasmin-Lebras ${ }^{1, *,+}$ \\ 1 SPEC, CEA, CNRS, Université Paris-Saclay, CEA Saclay, CEDEX, 91191 Gif-sur-Yvette, France \\ 2 Service de Pharmacologie et Immunoanalyse (SPI), Laboratoire d'Etudes et de Recherches en \\ Immunoanalyse, CEA, INRA, Université Paris-Saclay, 91191 Gif-sur-Yvette, France \\ 3 Direction des Programmes et des Partenariats Publics, Département de la Recherche Fondamentale, CEA, \\ 91191 Gif-sur-Yvette, France \\ * Correspondence: guenaelle.jasmin-lebras@cea.fr \\ $\dagger$ These authors contributed equally to this work.
}

Received: 7 August 2019; Accepted: 29 August 2019; Published: 31 August 2019

\begin{abstract}
Inexpensive simple medical devices allowing fast and reliable counting of whole cells are of interest for diagnosis and treatment monitoring. Magnetic-based labs on a chip are one of the possibilities currently studied to address this issue. Giant magnetoresistance (GMR) sensors offer both great sensitivity and device integrability with microfluidics and electronics. When used on a dynamic system, GMR-based biochips are able to detect magnetically labeled individual cells. In this article, a rigorous evaluation of the main characteristics of this magnetic medical device (specificity, sensitivity, time of use and variability) are presented and compared to those of both an ELISA test and a conventional flow cytometer, using an eukaryotic malignant cell line model in physiological conditions (NS1 murine cells in phosphate buffer saline). We describe a proof of specificity of a GMR sensor detection of magnetically labeled cells. The limit of detection of the actual system was shown to be similar to the ELISA one and 10 times higher than the cytometer one.
\end{abstract}

Keywords: diagnostic; GMR sensor; whole cell

\section{Introduction}

Routine diagnosis, treatment monitoring and treatment choice would greatly benefit from inexpensive and easy to use versatile devices capable of counting a small amount of whole cells of interest (cancer cells or bacteria) in different matrices [1-4]. A good example of the lack of adapted screening is breast cancer. Indeed, while it can be cured if detected early, it remains the most lethal cancer for women in developed countries [5].

The flow cytometry, developed since 1965 [6], is the gold standard for whole cell study and labeling. It continues to improve and allows for an increasing number of biological characterizations. The understanding of many biological processes like immune response or cell cycle, the screening of drug efficacy and the enrichment of the data bank about antigen distribution in different cell types are some examples of the huge impact of this technology on the field of fundamental biology [7]. In addition to its wide and growing interest for research purpose, flow cytometry has become routinely used in clinical laboratories over the last 20 years for different pathologies [7-9]. This technology is mainly applied in hematology but also in immunology and oncology. For these different disciplines, flow cytometry is used with different purposes like diagnosis, prognosis or treatment monitoring. The main advantages of this technology are the sensitivity and its possibility to measure simultaneously 
more than 20 parameters per cell [7]. It can also be applied to any cell type from bacteria to circulating tumor cells and to many different biological matrices (blood, bone marrow, solid tissues etc.) [8]. However, this technology still has some drawbacks preventing it from its use for clinical purposes: (i) these devices remain costly, bulky, the data treatment need skilled professionals and cannot be efficiently automated, (ii) the use of fluorescent antibodies makes this technique even more expensive and technically heavy as it necessitates washing steps and (iii) flow cytometry was not developed primarily to count cells but rather to recognize certain cell sub-populations. Some cytometers can give an absolute cell count but discrepancies between devices are common [10].

For early diagnosis purpose and the quantification of disease markers, several other techniques are already available in hospitals and clinical laboratories. Most of detection techniques like mass spectrometry, Western blots or techniques based on Polymerase Chain Reaction require lysis of cells to work with their inner DNA or proteins. In the context of whole cell study however, methods detecting specifically cells by their membrane markers are more relevant. The ELISA test (enzyme-linked immunosorbent assay), largely used, is typically applied to determine the concentration of molecular species in a suspension [11]. This technique is easily transposed to antigens expressed at cell surface as it has been done previously with bacteria [12]. The advantage of ELISA test is its simplicity, high throughput and relative low cost. However, this test still requires trained staff and requires relative long incubation time to reach its nominative performance (for instance the accuracy and reproducibility of the results depend on the reaction time [13]).

Several methods of easy to use cheap sensitive cell counters are being developed by numerous research groups. The development of micro-technologies for biological studies has paved the way for the creation of new devices relying on different detection systems for fast diagnosis. Different solutions are being developed to miniaturize flow cytometers and to simplify its use [14,15]. Optical detection is extremely performant but it requires lasers, precise alignments and interference with some matrices is a common problem either due to their auto-fluorescence or because of solubility issues [16]. Thus, alternative electrochemical or magnetic measurements using simpler detection systems have been proposed and even commercialized [17-22]. However these static methods imply washing steps and are conceived for proteins, DNA, RNA or small bacteria detection rather than for eukaryotic cells [23-26]. Moreover, non-specific interactions remain numerous with this type of devices, leading to reduced sensitivity $[27,28]$. Taking advantage of the high sensitivity of superconducting quantum interference device (SQUID) or fluxgates, several groups developed static techniques based on magnetorelaxometry [29-31]. These tools eliminate the need for washing steps as they discriminate free from bonded magnetic labels. However, SQUID operate at low temperature and their production is quite expensive [32]. Fluxgate-based techniques recently offered proof of their potential by detecting C-reactive protein in serum in $30 \mathrm{~min}$. Still, the electronic circuitry needed for results analyze must be further miniaturized [33]. Other approach are developed in parallel with simpler, less sensitive sensors. Dynamic methods using magnetic detection have been developed first on ferrofluid droplets [34] and raised an increasing interest since Loureiro et al. showed the ability of such devices to detect magnetic objects one by one [35,36] and thus their potential to reach an extremely low detection limit [37]. In addition, the sample can be prepared and tested without any washing step because of the dynamic magnetic detection, insensitive to matrix optical properties [38]. Nevertheless, if washing steps were needed anyway (eg. complex matrices or sample concentration requirements), the system could still offer this possibility as washings can be performed easily with the use of a simple permanent magnet allowing to immobilize beads from the matrix and which is a field-compatible method.

Several methods of magnetic detection have been proposed based on magnetic resonance effect, susceptibility measurements, giant magnetoimpedance (GMI), Hall Effect, Tunnel Magneto Resistance effect (TMR) or Giant Magneto Resistance effect (GMR) [24,39-44]. As biological objects are not magnetic and cannot be detected alone using magnetic sensors, the target must first be bound to magnetic particles (MPs or beads). This is possible thanks to antibodies (Abs), whose MPs are coated with, recognizing the target. The very high specificity of antibodies provides an easy way to target 
precisely the analyte of interest. Moreover the production of polyclonal as well as monoclonal antibodies (mAbs) directed against a given target is now a well-handled procedure in biology labs $[45,46]$. In a typical magnetic detection process, the mixture of the sample and mAbs-coated MPs is introduced into a microchannel where it flows above the sensors that detect the passage of magnetically labeled biological objects. Several groups worked with GMI sensors, using superparamagnetic particles and Helmoltz coils to generate the AC signal [42,43]. The use of GMR sensors is also a convenient choice for small objects detection due to their high sensitivity and their ease of production [25,39,47-50]. GMR sensors can now be produced industrially and their size tuned to match the target's and thus optimize the sensitivity. Moreover, they do not need an AC field to detect the passage of magnetic beads and thus their instrumentation can be simple.

Although several very interesting developments of this technique have been achieved, some difficulties remain [25]. In particular, the binding of the MPs to the target implies mixing the sample with a highly concentrated beads suspension to ensure that the target will meet and bind MPs in a reasonable time. Consequently a lot of free unbound MPs will linger in solution. Moreover, when the target analyte is a living cell, there is inevitably a discrepancy of distribution of the number of magnetic beads bound to each cell. This is due to the natural distribution variation of the number of epitopes per cell recognized by mAbs. Since MPs tend to agglomerate in physiological conditions, the signals created by cells have to be compared not only to those created by single MPs but also to those created by MP aggregates whose sizes depends on the bead type and concentration and on the matrix used. Furthermore, as the signal amplitude depends greatly on the distance between the object and the sensor, it is possible that a small aggregate of beads, moving above the sensor at a short distance gives the same signal as a biological object covered with numerous MPs but flowing further above it. In an attempt to overcome this limit, some ideas have been recently proposed. One consists in using flow focusing to concentrate the detected objects in the bottom half of the channel and avoid this uncertainty [51-53]. Yet, while screening tools must remain simple, the use of flow focusing adds a sheath fluid whose flow must be judiciously adjusted. Another idea relying on chip design combining mechanical and magnetophoretic guiding has been proposed to drag all magnetic material at the bottom of the channel without the need of sheath fluid. This method requires precise adaptation to each system and has not been evaluated on any biological model yet [54].

In this work, we suggest a third technique to discriminate specific signals from aggregates, consisting in heightening the floor of the channel above the magnetic sensors so that single beads or small aggregates cannot be detected. We present a complete and reliable process of detection, including negative controls to evaluate specificity, a sensitivity study and a variability evaluation. We have developed a magnetoresistive cell counting device using murine myeloma cells as a biological model. The results have been compared with two standard methods of detection mentioned previously, a microplate sandwich ELISA immunoassay and flow cytometry using the same reagents (mAbs, buffer, samples), which is the only reliable way to compare accurately methods. Similar performances were obtained for the ELISA test and the GMR test while flow cytometry obtained a ten times lower limit of detection.

\section{Materials and Methods}

\subsection{Sensor Fabrication}

The spin valve layers are deposited on a $300 \mu \mathrm{m}$ thick silicon wafer. The thin films arrangement can be described as follows: $\mathrm{Ta}(3) / \mathrm{Ni}_{80} \mathrm{Fe}_{20}$ (3.5)/ $\mathrm{Co}_{90} \mathrm{Fe}_{10}(1.5) / \mathrm{Cu}(2.3) / \mathrm{Co}_{90} \mathrm{Fe}_{10}(2.1) / \mathrm{Ru}(0.85) / \mathrm{Co}_{90}$ $\mathrm{Fe}_{10}(2.0) / \mathrm{Pt}_{50} \mathrm{Mn}_{50}(18) / \mathrm{Ta}(3) / \mathrm{Ru}(3)$ where the thickness of layers is given in nanometers and the target composition is given in percentages. The sensors are then patterned by UV photolithography in a positive resin $\mathrm{S} 1805$ and then etched by ion beam etching (IBE). The contact pads are deposited by evaporation of a bilayer $\mathrm{Ti}(3 \mathrm{~nm}) / \mathrm{Au}(100 \mathrm{~nm})$, after having been designed by photolithography in $\mathrm{S} 1813$ positive resin. Finally, a passivation bilayer of $150 \mathrm{~nm}$ thick $\mathrm{Al}_{2} \mathrm{O}_{3}$ and $150 \mathrm{~nm}$ thick $\mathrm{Si}_{3} \mathrm{~N}_{4}$ 
are deposited by sputtering on the whole chip surface except on the contact pads. The usual sensor resistance was around $600 \Omega$. This passivation layer insures a good lifetime of the sensors in aggressive matrices.

\subsection{Microfluidic Channel Fabrication}

The microfluidic channel has been realized by using a classical protocol [55]. A layer of PDMS of an expected thickness of $6 \mu \mathrm{m}$ is spin-coated ( $5 \mathrm{~min}, 2700 \mathrm{rpm}, 300 \mathrm{rpm} / \mathrm{s}$ ) on the sensors after a plasma $\mathrm{O}_{2}$ treatment $(15 \mathrm{~s}, 40 \mathrm{~mW}, 0.1 \mathrm{mbar})$ to improve the adhesion. The device is then heated at $110{ }^{\circ} \mathrm{C}$ during $20 \mathrm{~min}$ and at $60^{\circ} \mathrm{C}$ at least for $45 \mathrm{~min}$. In parallel, the $25 \mu \mathrm{m}$ high and $100 \mu \mathrm{m}$ wide PDMS channel was molded over an SU-8 mold obtained by UV photolithography and measured by a mechanical profilometer (Alpha-Step, KLA Tencor, Mipitas, CA, USA). After demolding, the injection holes are made in the PDMS using a puncher. After the same aforementioned plasma treatment, the channel is aligned above the sensor using an MJB4 aligner and put in contact with the substrate. The chips are then heated for $20 \mathrm{~min}$ at $120^{\circ} \mathrm{C}$ and for $1 \mathrm{~h}$ at $60^{\circ} \mathrm{C}$.

\subsection{Cell Culture}

Two cell lines were used for the study: first, the NS1, murine myeloma cells, showing an average diameter of $6 \mu \mathrm{m}$ and expressing at their surface the CD138 protein (Syndecan-1) and second, the Chinese Hamster Ovary cells (CHO) with an average diameter of $10 \mu \mathrm{m}$ that do not express the CD138 protein. The cell culture media were from Gibco ${ }^{\circledR}$, Life Technologies, Carlsbad, CA, USA.

NS1 cells were cultivated in Dulbecco's medium with $15 \%$ of fœtal bovine serum, $1 \%$ of non-essential amino acids, $1 \%$ of antibiotics (penicillin and streptomycin) and $1 \%$ of $\mathrm{L}$-glutamine at $37^{\circ} \mathrm{C}$ under a controlled atmosphere containing $7 \%$ of $\mathrm{CO}_{2}$. They were centrifuged at $1000 \mathrm{RPM}$ (centrifuge diameter $344 \mathrm{~mm}$ ) for $10 \mathrm{~min}$ at $9^{\circ} \mathrm{C}$ and then diluted in PBS (Dulbecco's Phosphate Buffer Saline, Gibco, Life Technologies) in which the tests were carried out.

CHO cells were cultivated in Ham F-12 Nutrient Mixture with 10\% of fœtal bovine serum, $1 \%$ of non-essential amino acids, $1 \%$ of antibiotics (penicillin and streptomycin) and $1 \%$ of $\mathrm{L}$-glutamine at $37^{\circ} \mathrm{C}$ under a control atmosphere containing $5 \%$ of $\mathrm{CO}_{2}$. They were washed two times in PBS, let in a solution of $0.25 \%$ trypsin-EDTA for $5 \mathrm{~min}$ at $37^{\circ} \mathrm{C}$ and were centrifuged at $1000 \mathrm{RPM}$ (centrifuge diameter $344 \mathrm{~mm}$ ) for $5 \mathrm{~min}$ at $9{ }^{\circ} \mathrm{C}$. Finally, they were diluted in PBS before use.

\subsection{Production of IpaD-315 Antibodies}

Six to 8-week-old female BALB/c mice were purchased from Janvier Labs, France and maintained in accordance with the French and European regulations on care and protection of laboratory animals (European Community [EC] Directive 86/609, French Law 2001-486, 6 June 2001) and with agreement of the ethical committee (CETEA) no. 15-055 delivered to S. Simon and agreement D-91-272-106 from the Veterinary Inspection Department of Essonne (France). Up to eight mice were kept in each cage and housed in a temperature-regulated-room and had free access to food and water. All animals' experiments were performed to minimize suffering according to the guideline of the CETEA committee. IpaD-315 murine monoclonal antibody was produced in the LERI laboratory (SPI/CEA Saclay, France). It was raised in BALB/c mice by repeated intranasal immunizations with $20 \mu \mathrm{g}$ of purified recombinant IpaD protein expressed in E. coli BL21DE(3) [56]. Ipad gene was amplified from Shigella flexneri (CIP $82.48 \mathrm{~T}$ ) and cloned into the IPTG inducible pET22b(+) vector (Novagen) allowing insertion of a poly-histidine tag sequence at the $3^{\prime}$ end of the gene used for protein purification. Hybridomas were produced by fusing spleen cells of immunized mice with NS1 myeloma cells, according to Köhler and Milstein [45]. IpaD-315 monoclonal antibody was then produced in ascite fluids in BALB/C mouse and further purified by protein A affinity chromatography. The purity of IpaD-315 mAb was assessed by SDS-PAGE in reducing and non-reducing conditions and its isotype determination was performed using Pierce rapid ELISA mouse antibody isotyping kit (Thermo Scientific). 


\subsection{Particle Functionalization}

Dynabeads My One Streptavidin T1 were selected. They are $1 \mu \mathrm{m}$ diameter homogeneous polymer particles embedding superparamagnetic iron oxide nanoparticles. They have been functionalized with two different monoclonal antibodies of the same IgG2a isotype: a rat anti-CD138 mAb (BD Pharmingen) and a murine mAb, IpaD-315 (described in Section 2.4), according to the commercial protocol after their biotinylation and purification.

For mAb biotinylation, $100 \mu \mathrm{g}$ of antibodies were diluted in $400 \mu \mathrm{L}$ of $0.1 \mathrm{M}$ borate buffer $\mathrm{pH}$ 9.0 containing $6 \mu \mathrm{L}$ of biotin (Sigma-Aldrich) in DMF at $1 \mathrm{mg} / \mathrm{mL}$ and incubated for $30 \mathrm{~min}$ at room temperature. Then, $100 \mu \mathrm{L}$ of $1 \mathrm{M}$ Tris $\mathrm{HCl}$ buffer $\mathrm{pH} 8.0$ were added and incubated for $15 \mathrm{~min}$. Finally, the biotinylated $\mathrm{mAb}$ was purified from free biotin on Zeba Desalt Spin column (Thermo Scientific) in $0.1 \mathrm{M}$ potassium phosphate buffer $\mathrm{pH} 7.4$ with $0.15 \mathrm{M} \mathrm{NaCl}$. The absorbance of the final solution was measured between 280 and $320 \mathrm{~nm}$ to determine the concentration of the purified biotinylated antibody. Biotinylated antibodies were then mixed at room temperature with streptavidin coated beads for $30 \mathrm{~min}$, washed four times in PBS 0.1\% BSA and stored in PBS $0.1 \%$ BSA at $4{ }^{\circ} \mathrm{C}$ until use.

\subsection{MP Cell Labeling}

Several cell concentrations have been used: $10^{5}, 310^{4}, 10^{4}, 310^{3}$ and $10^{3}$ cells $/ \mathrm{mL}$ while the MP concentration was set to $23 \mu \mathrm{g} / \mathrm{mL}$ corresponding to $210^{7}$ antibodies-coated beads per milliliter. Indeed, the beads concentration must be independent of the cell concentration as this value is unknown in a real sample. In addition to the positive samples with the targeted MP-labeled cells with concentrations described above, three negative samples were prepared and used in experiments: (i) $1 \mathrm{~mL}$ of buffer containing only the $23 \mu \mathrm{g}$ of beads functionalized with anti-CD138 antibody, (ii) 1 $\mathrm{mL}$ of buffer containing $10^{5} \mathrm{NS} 1$ cells and $23 \mu \mathrm{g}$ of the beads functionalized with control IpaD-315 antibody and (iii) $1 \mathrm{~mL}$ of buffer containing $10^{5} \mathrm{CHO}$ cells and the $23 \mu \mathrm{g}$ of beads functionalized with anti-CD138 antibody. Indeed, the detection of typical signals does not mean necessarily that a myeloma cell has been detected: it could also be an aggregate of beads or some MPs bound via non-specific interactions on another kind of cells. A comparison with negative samples is thus needed. The Table 1 summarizes the samples used.

Table 1. Summary of used samples.

\begin{tabular}{lcc}
\hline Sample Type & Cells/mL & Antibody Beads Coating \\
\hline & $1 \times 10^{5} \mathrm{NS1}$ & anti-CD138 \\
& $3 \times 10^{4} \mathrm{NS1}$ & anti-CD138 \\
Positive & $1 \times 10^{4} \mathrm{NS1}$ & anti-CD138 \\
& $3 \times 10^{3} \mathrm{NS1}$ & anti-CD138 \\
& $1 \times 10^{3} \mathrm{NS1}$ & anti-CD138 \\
\hline & $1 \times 10^{5} \mathrm{NS1}$ & IpaD315 \\
Negative & $1 \times 10^{5} \mathrm{CHO}$ & anti-CD138 \\
& No cell & anti-CD138 \\
\hline
\end{tabular}

The cells of each sample have been counted at the beginning of each experiment to check the nominal concentration using a Malassez cell. After mixing the MPs with cell suspensions, the samples were incubated at room temperature under a slow rotation for two hours.

\subsection{Experimental Set-Up}

In an experiment, superparamagnetic objects (labeled cells, unbound MPs and MPs aggregates) magnetized by a permanent magnetic field are flowing above the sensor in a microfluidic channel. The magnetic field must be as homogeneous as possible. Indeed, magnetic gradients, by exerting locally a magnetic force on the particles, can lead to local accumulation of beads in the channel and even clog it. The chips and the inlet and outlet reservoirs are thus inserted in the permanent field created 
by two ferrite magnets of $3 \times 3 \times 10 \mathrm{~cm}^{3}$ on sides closed with two soft $8 \mathrm{~mm}$ iron sheets on top and bottom (see Figure 1a). Using this device, the magnetic field varies by less than $1 \mathrm{mT}$ over the entire surface of the chip (which is $1.5 \mathrm{~cm}$ long by $5 \mathrm{~mm}$ wide) (see Figure $1 \mathrm{~b}$ ), while the vertical magnetic field reaches $90 \mathrm{mT}$. The chip is fixed on a support whose angles can be finely tuned to maximize the sensor sensitivity (see Figure 1c). The sensitivity is maximal when the external field is rigorously perpendicular to the sensor surface. At the beginning of each experiment, the position of the sensor is set using a calibrated coil fixed on the magnet which generates a $1 \mathrm{kHz}$ in-plane reference magnetic signal. The aim of this positioning is to maximize the sensitivity and to minimize the noise of the resulting signal. Indeed, the precise location of the sensor influences the random telegraphic noise that appears in some configurations. Then, these two characteristics are measured. The smallest detectable signal, called threshold, is defined as having an amplitude exceeding three times the noise level. In a typical experiment, the sensor noise was evaluated at $50 \mathrm{nT} / \sqrt{\mathrm{Hz}}$, there was $6 \mu \mathrm{V}$ of noise on the whole bandwidth of $15 \mathrm{kHz}$ and the sensor sensitivity was $2.5 \% \cdot \mathrm{mT}^{-1}$.

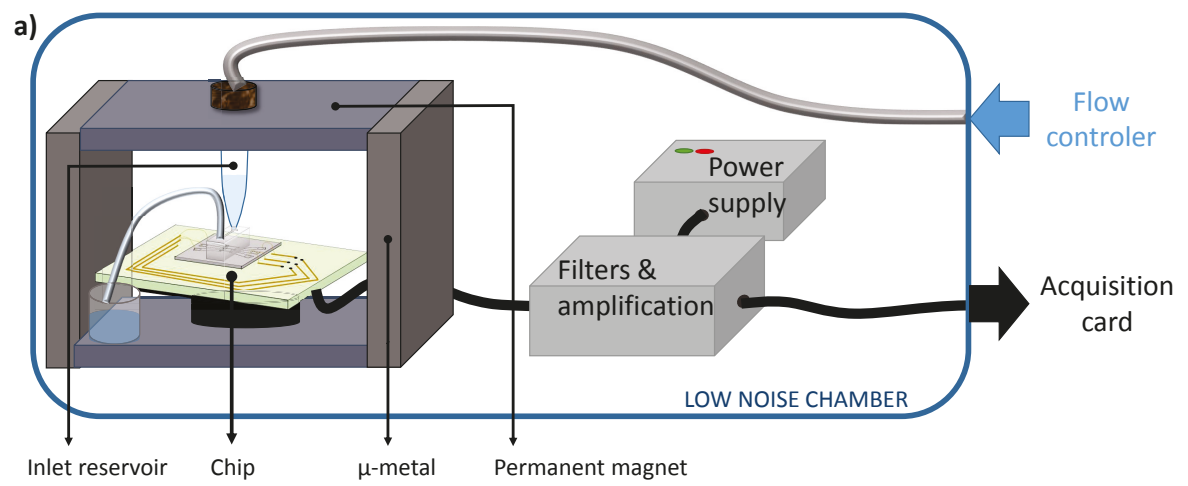

b)

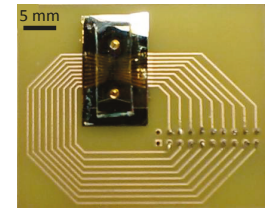

c)

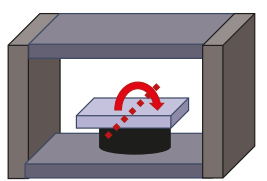

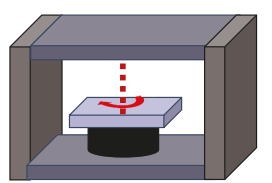

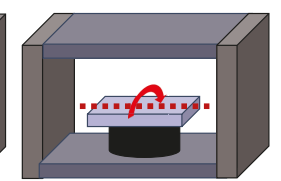

Figure 1. Experimental set-up and data. (a) The chip, the reservoir and the collecting vial are inserted in a homogeneous magnetic field. A computer program is controlling the flow using a pressure driver. The applied pressure is set to 300 mbar. Homemade electronic boxes deliver power to the sensor, amplify and filter the signals before sending the data outside the low-noise chamber to the acquisition card. (b) Chip photograph. (c) Positioning angles.

The device and electronic boxes were used in a magnetically shielded room $\left(2.9 \times 2.9 \times 2.3 \mathrm{~m}^{3}\right)$ made of three $\mu$-metal layers and three aluminum layers. In this environment, the noise level is of $1 \mathrm{nT} \sqrt{\mathrm{Hz}}$ which is low compared to the intrinsic sensor noise. In a real commercial device, a reference GMR sensor (outside of the microfluidic channel) is enough to substract environmental noise, mainly the 50 or $60 \mathrm{~Hz}$ magnetic field created by power lines as it has already been done by some groups [57,58].

The flow is driven by a pressure controller (MFCS ${ }^{\mathrm{TM}}$-EZ: Microfluidic Flow Control System, Fluigent ${ }^{\circledR}$ ) and the pressure is set to 300 mbar, typically a sample of $1 \mathrm{~mL}$ is flowed in $30 \mathrm{~min}$. The liquid sample is directly injected at the top of the inlet reservoir, made of polyoxymethylene to minimize cells and beads adhesion on its walls. This reservoir is set in vertical position to insure that sedimentation would not impede some cells to go into the channel. The wet part of the reservoir is 
completely localized in the gap between the two magnets to minimize magnetic forces exerted on the content.

\subsection{Electronics}

The electronics is battery supplied to avoid $50 \mathrm{~Hz}$ noise. The sensors are biased at voltages between 1 to $2 \mathrm{~V}$ and the output signal is amplified 500 times by a low noise preamplifier and filtered at $15 \mathrm{kHz}$ with an additional gain of 20. The signal is then oversampled at $200 \mathrm{kHz}$ using a Data Translation ${ }^{\circledR}$ acquisition card controlled by a homemade software. A schematic view of this set-up is presented in Figure 1a. Then, a homemade software identifies the signals from the total recording and discriminate them from noise artifacts. Numerical parameters were evaluated on a cohort of several thousands of examples from different experiments. For each spotted point above the threshold, the local minimum and maximum are determined by repeatedly incrementing the interval of interest by 15 points until the maximum $\left(i_{\text {max }}, V_{\text {max }}\right)$ and minimum $\left(i_{\text {min }}, V_{\text {min }}\right)$ determined are more than 20 points from each edge of the interval. The user determines the direction of signals $\left(k=1\right.$ if $i_{\max }-i_{\min }<0$ and $k=-1$ if $\left.i_{\max }-i_{\min }>0\right)$ and the detection threshold $\left(V_{t h r}\right)$. Several checks are then carried out to validate the recording of this peak in the processed file. They must be bipolar $\left(\left|V_{\min }\right|>1 / 3 V_{\text {thr }}\right.$ and $\left.\left|V_{\max }\right|>1 / 3 V_{\text {thr }}\right)$ with the right orientation $\left(k\left(i_{\max }-i_{\min }\right)<0\right)$, their width $\left(i_{\max }-i_{\min }\right)$ must be coherent with the flow velocity (between $25 \mu$ s and $2.5 \mathrm{~ms}$ ) and they must be sufficiently symmetric $\left(\left|\frac{V_{\max }-V_{\min }}{V_{\max }+V_{\min }}\right|<0.4\right)$. This last criteria was added to better discriminate signals from radiotelegraphic noise occurring in some experiments. Experimental data before and after treatment are presented respectively in Figure 2a,b.

a)

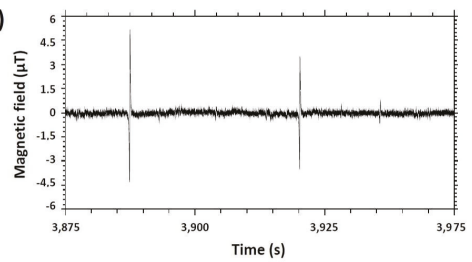

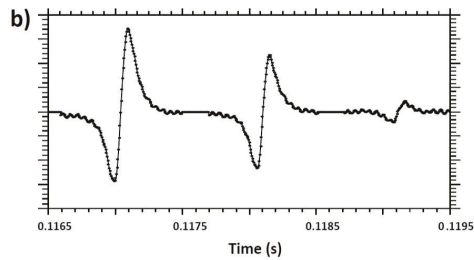

Figure 2. Experimental data. (a) Raw experimental recording. (b) Recording of the software-selected portions (the same 3 signals are shown).

\subsection{Comparative ELISA Tests}

96 wells plates were coated with anti-CD138 antibody. In each well, $100 \mu \mathrm{L}$ of a suspension of 10 $\mu \mathrm{g} / \mathrm{mL}$ of antibodies in potassium phosphate buffer at $50 \mathrm{mM}, \mathrm{pH} 7.4$ were deposited and incubated overnight at $20^{\circ} \mathrm{C}$. The following day, wells were emptied and filled with $300 \mu \mathrm{L}$ of EIA buffer (100 $\mathrm{mM}$ potassium phosphate buffer $\mathrm{pH} 7.4$ containing $0.1 \%$ bovine serum albumin, $0.15 \mathrm{M} \mathrm{NaCl}$ and $0.01 \%$ sodium azide). The plates were sealed and stored at $4{ }^{\circ} \mathrm{C}$ until use.

The day of the experiment, the coated plate was washed once in a washing buffer $(50 \mathrm{mM}$ potassium phosphate buffer $\mathrm{pH} 7.4), 100 \mu \mathrm{L}$ of serial dilutions of NS1 cells $\left(310^{6} ; 10^{6} ; 310^{5} ; 10^{5} ; 3\right.$ $10^{4} ; 10^{4} ; 310^{3} ; 10^{3}$ cells $/ \mathrm{mL}$ ) in PBS were added per well and incubated under agitation at room temperature for $2 \mathrm{~h}$. Then, the plate was washed three times in the washing buffer and $100 \mu \mathrm{L}$ of a suspension of biotinylated antibody anti-CD138 at $200 \mathrm{ng} / \mathrm{mL}$ in EIA buffer without sodium azide were added per well for a $2 \mathrm{~h}$-incubation step under agitation at room temperature. The plate was then washed three times in the washing buffer and $100 \mu \mathrm{L}$ of a solution of streptavidin conjugated with polymers of horseradish peroxidase (Thermofisher Scientific, Waltham, MA, USA) diluted 15,000 fold in EIA buffer without azide was added into the wells. Finally, after 30 min of incubation under agitation at room temperature, the plate was washed 5 times in the washing buffer and $100 \mu \mathrm{L}$ of $3,3^{\prime}, 5,5^{\prime}$-Tetramethylbenzidine (TMB, Thermofisher Scientific) were added per well. After 30 min at room temperature under agitation, $100 \mu \mathrm{L}$ of $2 \mathrm{M}$ sulfuric acid were added per well and the absorbance 
of each well was measured at $450 \mathrm{~nm}$ (wavelength of absorption of the reaction product) and $620 \mathrm{~nm}$ (noise measurement).

The substraction of these two measurements yields the specific signal directly proportional to NS1 cell concentration. The theoretical limit of detection is defined as the lowest cell concentration giving a signal greater than the non-specific binding (mean of eight measurements of EIA buffer) +3 standard deviations ( $99.7 \%$ confidence). The theoretical limit of quantification is defined as the lowest cell concentration giving a signal greater than the non-specific binding (mean of eight measurements of EIA buffer) + 10 standard deviations ( $99.9 \%$ confidence) .

\subsection{Comparative Flow Cytometry Tests}

For flow cytometry analysis, NS1 cells were washed once with PBS/0.5\% BSA and $200 \mu \mathrm{L}$ of serial dilutions of cells $\left(10^{5} ; 310^{4} ; 10^{4} ; 310^{3} ; 10^{3}\right.$ cells $\left./ \mathrm{mL}\right)$ were incubated for $2 \mathrm{~h}$ at $4{ }^{\circ} \mathrm{C}$ with anti-mouse CD138 labeled with Phycoerythrin (BD Biosciences). After incubation, cells were washed twice with PBS $/ 0.5 \%$ BSA and resuspended in $200 \mu \mathrm{L}$ of PBS $/ 0.5 \%$ BSA. The fluorescence was finally assayed for the total volume of $200 \mu \mathrm{L}$ using a Novocyte flow cytometer (ACEA) and the number of stained cells was evaluated by comparison with cells incubated with buffer alone. Results were analysed using NovoExpress software.

\section{Results and Discussion}

\subsection{Simulations of Single Magnetic Beads and MP-Labeled Cells}

A GMR sensor is composed of two ferromagnetic metallic layers separated by a nonmagnetic one as shown in Figure 3a. The magnetization of one of these layers is pinned in one direction while the magnetization of the other one is free to rotate in its plane. As the speed of propagation of electrons in a metal strongly depends on the relative orientation of its spin and the magnetization of the metal, this spintronic device will have different properties for spin up and spin down electrons. Depending on the angle between the magnetizations of the two ferromagnetic layers, the overall resistance of the sensor varies as shown in Figure 3b. During the detection process, the MPs themselves are magnetized perpendicularly to the sensor plane by a field created with a permanent magnet and emit a dipolar field. Only the in-plane component of the dipolar field created by the beads is detected by the magnetic sensor since thin-film GMR sensors are insensitive to out-of-plane field variations (z direction) below a critical value. The sensor yoke geometry, designed especially to have just one magnetic domain [59], is shown in Figure $3 \mathrm{~d}$ and presents a high aspect ratio. This strong asymmetry between the length and width of the device will tend to align all the moments from the free layer according to its length to reduce the magnetostatic energy by moving the two poles created as far as possible from each other. The free layer magnetization is thus along the $\mathrm{x}$ axis at zero field, while the other layer is pinned along the $y$ axis. The device is in its most sensitive configuration at zero field and is sensitive only to the $y$ component of the field. Moreover, improving the alignment of moments from the free layer results in a more linear behavior of the sensor. GMR sensors response to small magnetic fields variations are linear on a range of about $\pm 2 \mathrm{mT}$ around zero field (see Figure $3 \mathrm{~b}$ ), which includes the whole range of fields needed for this application.

The situation to be modeled is presented in Figure 3c. Magnetized objects circulate above the sensor in a laminar flow in a microchannel and induce magnetic field variations that are detected by the sensor. Three types of magnetic objects are modeled: single magnetic beads, aggregates of beads and MPs-labeled cells. 
a)

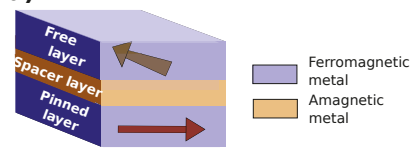

b)

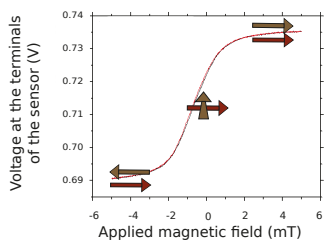

c)
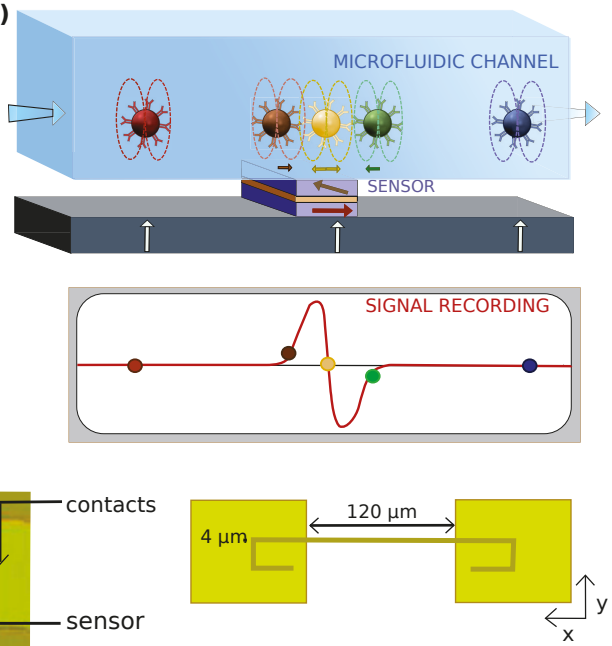

Figure 3. Giant magnetoresistance (GMR) sensor. (a) Scheme of the main components of a GMR stack. Free and pinned layers are ferromagnetic and their magnetization are represented by arrows. The spacer is a diamagnetic conductor. (b) Experimental sensitivity curve with schematic representation of the relative orientations of the two ferromagnetic layers. This sensor shows a sensitivity of $2 \% \cdot \mathrm{mT}^{-1}$ and no hysteresis on its linear portion. (c) Schematic of the experiment: Labeled objects are moved by the laminar flow at a given height crossing the sensor at constant speed. The sensor detects variations of the magnetic field due to the induced dipolar field of the beads. The beads are magnetized by a field normal to the sensor plane created by a permanent magnet. (d) Photograph and scheme of a processed GMR sensor in yoke shape. The sensor measures $120 \mu \mathrm{m}$ along the $\mathrm{x}$ axis and $4 \mu \mathrm{m}$ along the $\mathrm{y}$ axis.

The signal corresponding to a single bead moving above the sensor is proportional to the integral over the whole sensor surface of the $y$-component of the local dipolar field induced at each successive position. For a MP in position $\left(x_{B}, y_{B}, z_{B}\right)$ with a moment $\vec{\mu}$ making an angle $\theta$ with $\vec{z}$ and $\phi$ with $\vec{x}$ and moving above a sensor of length $L$ and width $l$, it is given by the Formula (1) [60].

$$
\begin{aligned}
H_{y} & =\frac{\mu}{L l}\left(\left(\frac{y_{l}}{q_{2}^{2}}\left(\frac{x_{r}}{r_{2}}-\frac{x_{l}}{r_{1}}\right)+\frac{y_{r}}{q_{4}^{2}}\left(\frac{x_{l}}{r_{4}}-\frac{x_{r}}{r_{3}}\right)\right) \sin \theta \sin \psi\right. \\
& +\left(\frac{1}{r_{1}}-\frac{1}{r_{2}}+\frac{1}{r_{3}}-\frac{1}{r_{4}}\right) \sin \theta \cos \psi \\
& \left.+\left(\frac{h}{q_{2}^{2}}\left(\frac{x_{l}}{r_{1}}-\frac{x_{r}}{r_{2}}\right)+\frac{h}{q_{4}^{2}}\left(\frac{x_{r}}{r_{3}}-\frac{x_{l}}{r_{4}}\right)\right) \cos \theta\right)
\end{aligned}
$$

where $x_{r}, x_{l}, y_{r}, y_{l}, h, r_{1}, r_{2}, r_{3}, r_{4}, q_{2}$ and $q_{4}$ are defined as follows.

$$
\begin{aligned}
& x_{r}=\frac{L}{2}-x_{B} \\
& y_{r}=\frac{l}{2}-y_{B} \\
& h=z_{B}-z_{C} \\
& r_{1}=\sqrt{x_{l}^{2}+y_{l}^{2}+h^{2}} \\
& r_{3}=\sqrt{x_{r}^{2}+y_{r}^{2}+h^{2}} \\
& q_{2}=\sqrt{y_{l}^{2}+h^{2}}
\end{aligned}
$$

$$
\begin{aligned}
& x_{l}=-\frac{L}{2}-x_{B} \\
& y_{l}=-\frac{l}{2}-y_{B} \\
& r_{2}=\sqrt{x_{r}^{2}+y_{l}^{2}+h^{2}} \\
& r_{4}=\sqrt{x_{l}^{2}+y_{r}^{2}+h^{2}} \\
& q_{4}=\sqrt{y_{r}^{2}+h^{2}}
\end{aligned}
$$


After several simulation tests (data not shown), it has been concluded that the influence of the spatial distribution of the moments in the aggregates was negligible. Signals from aggregates of $\mathrm{N}$ beads are simulated as $\mathrm{N}$ times the signals coming from a single bead with the same parameters.

On the contrary, the distribution of MPs on the cell surface was proven to have an influence on the generated signals, as presented in Figure $4 \mathrm{~b}$. Cells are thus simulated as spheres with several magnetic beads distributed randomly on their surfaces and with a random angle $\theta$ between the direction of their moment and the vertical axis with the constraint of a total magnetization equal to the experimentally measured one (see Section 3.2). This observation leads to the conclusion that detecting one passage with one single sensor cannot be sufficient to deduce precisely the nature and the details of the detected object.

a)

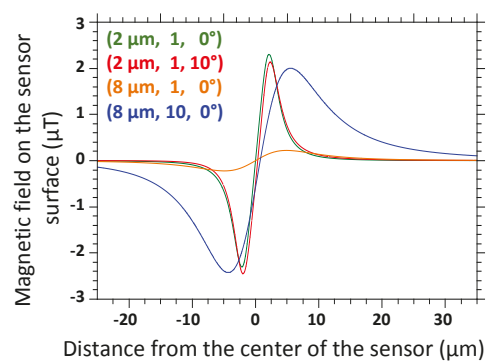

b)

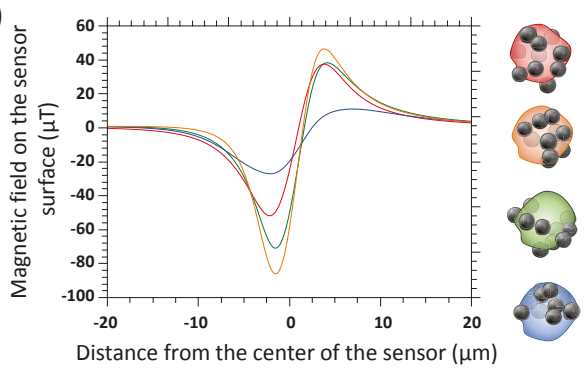

Figure 4. (a) Simulation results of the magnetic object detection demonstrating the influence of the three main parameters: distance between object and sensor (Z), number of magnetic particles (MPs) $(\mathrm{N})$ and moment orientation $(\theta)$. Curves are labeled by the triplet $(\mathrm{Z}, \mathrm{N}, \theta)$. (b) Simulation results for a 6 $\mu \mathrm{m}$ diameter cell at $6 \mu \mathrm{m}$ height covered by $10 \mathrm{MPs}$, with four sets of random positions of the beads on the cell surface.

However, our objective is to discriminate the MP-labeled cells from the aggregates of magnetic beads. Three experimental parameters must be chosen together to optimize the discrimination: (i) the chip design, (ii) the permanent magnet, (iii) the magnetic particles. Indeed, with fixed values for these three settings, the values of the two parameters determining the signal shape (the height of the magnetic object and its magnetic moment) are framed. The object distance from the sensor has the largest importance on the amplitude of the signal as shown on Figure 4a. As a consequence, to increase the impact of the number of MPs per object on the resulting signal (value correlated to the nature of this object), the object distance from the sensor needs to be the most homogeneous as possible, hence, the channel must be the smallest possible. This parameter was set to $25 \mu \mathrm{m}$, the lowest value at which the channel would not clog after $2 \mathrm{~h}$ of use. The permanent magnet must be chosen so that its field is sufficient to have a small average angle $\theta$ between the beads magnetic moments and the vertical axis but must be low enough not to pull the pinned layer of the sensor out of plane. This value was set at $90 \mathrm{mT}$ as it was a good compromise knowing the MPs magnetization curves. The choice of the magnetic beads and of the chip design are explained in the following paragraphs.

\subsection{Deduction of Best Experimental Conditions}

The chip design was optimized by testing different configurations of the sensor and microchannel geometries on simulated samples. The main idea was to make some static changes that would not complicate the use of the device but would enhance the discrimination between "positive" and "negative" samples. Samples are called "positive" if they are supposed to contain MPs-labeled cells (they contain a specific complex mAbs-coated beads/cells possessing antigens targeted by the mAbs) and "negative" if they are not, see Table 1. 


\subsubsection{Sample Characterization}

The two kinds of samples had thus to be experimentally characterized to make a proper model. Five sorts of commercial magnetic beads ranging from $200 \mathrm{~nm}$ to $2.8 \mu \mathrm{m}$ in diameter from three companies were tested for the preparation of these samples. Indeed, the choice of MPs is important. Ideal beads should have a magnetic moment sufficiently high to be attracted by a permanent magnet within few minutes to enable simple and quick washing steps (required for mAbs functionalization of MPs before performing the test). They should also have a low saturation field so that a permanent magnet of $90 \mathrm{mT}$ is enough to reach quasi-saturation and, above all, they must present the lowest propensity to aggregation. All these properties were investigated as follows.

First, the magnetic moment of $50 \mu \mathrm{L}$ of each of the MPs suspensions has been measured using a Vibrating Sample Magnetometer (VSM) and, using the manufacturer number of beads information, the saturation magnetic moment of a single bead has been calculated and the saturation field was determined.

Then, each type of beads was functionalized with antibodies. After this step, their kinetics of cell labeling and their aggregation were studied in parallel. Different concentrations of each type of MPs have been mixed in PBS and with $10^{5} \mathrm{NS} 1 / \mathrm{mL}$ in PBS. For 2 to $3 \mathrm{~h}$, regularly, $100 \mu \mathrm{L}$ of these suspensions were poured into a well and let to sediment for $15 \mathrm{~min}$. Pictures were then taken under optical microscope. For each cell-containing sample, the distribution of the number of beads per cell was evaluated by visual counting. For the samples without cells, hundreds of these photographs were analyzed with the ImageJ software. They were binarized before the software counted the number of pixels in each aggregate. From these data, the distribution of beads in aggregates was deduced.

Finally, Dynabeads MyOne Streptavidin T1 superparamagnetic have been chosen for the present study (Figure 5, results for the 4 other investigated types of beads can be found in Appendix A). They are $1 \mu \mathrm{m}$ polymer beads containing maghemite clusters and have a sufficiently high moment that they can be attracted by a permanent magnet within $2 \mathrm{~min}$, thus washing steps (required for mAbs functionalization of MPs) are extremely simple and quick. The saturation magnetic moment of a single bead has been calculated to be $2.110^{-11}$ emu per bead and reached at a field of $700 \mathrm{mT}$ but at $90 \mathrm{mT}$ these beads already have an average magnetic moment of $1.610^{-11}$ emu per bead (data not shown). They are easily functionalized with any kind of purified biotinylated antibodies with high efficiency, with the protocol described in Section 2.5. Moreover the number and size of bead aggregates in the commercial suspension were the lowest of the 5 studied MP types (93\% of the objects in the suspension are composed of less than 7 beads, $99 \%$ less than 15 beads, the complete curve is presented on Figure 5f) and the distribution of beads per cell was satisfying with an average of roughly 50 MPs per cell (the distribution and the 100 magnification photographs are shown on Figure 5).

\subsubsection{Chip Design}

The objective is to find the most favorable conditions to discriminate labeled cells from aggregates. As described in details in the Section 3.1, the signals are determined by the dipolar field created by the detected object which depends mainly on the magnetic moment of the object and its height from the sensor. The expression of the dipolar field $\overrightarrow{H_{\text {dip }}}$ created in B and sensed in $\mathrm{C}$ is given by Equation (2) where $\vec{\mu}$ is the magnetic moment of a bead and $N$ is the number of beads of the detected object.

$$
\overrightarrow{H_{d i p}}=3 \overrightarrow{B C} \cdot \frac{\overrightarrow{B C} \cdot N \vec{\mu}}{\|\overrightarrow{B C}\|^{5}}-\frac{N \vec{\mu}}{\|\overrightarrow{B C}\|^{3}}
$$

On the Figure $5 \mathrm{f}$, the distributions of beads in cells and in aggregates are given for our system. By using Equation (2), it can be calculated that, between the smallest object in negative samples (1 bead) and the most labeled cell (around 100 beads), the signal is multiplied by 100 . Between a height of $1 \mu \mathrm{m}$ from the sensor and of $10 \mu \mathrm{m}$ from the sensor, the signal is divided by 1000 . Thus, when the channel is directly placed at the top of the sensor, even a signal from a single bead (at $1 \mu \mathrm{m}$ height) cannot be 
discriminated from a signal coming from a labeled cell further away from the sensor in the channel, independently of the detection threshold.

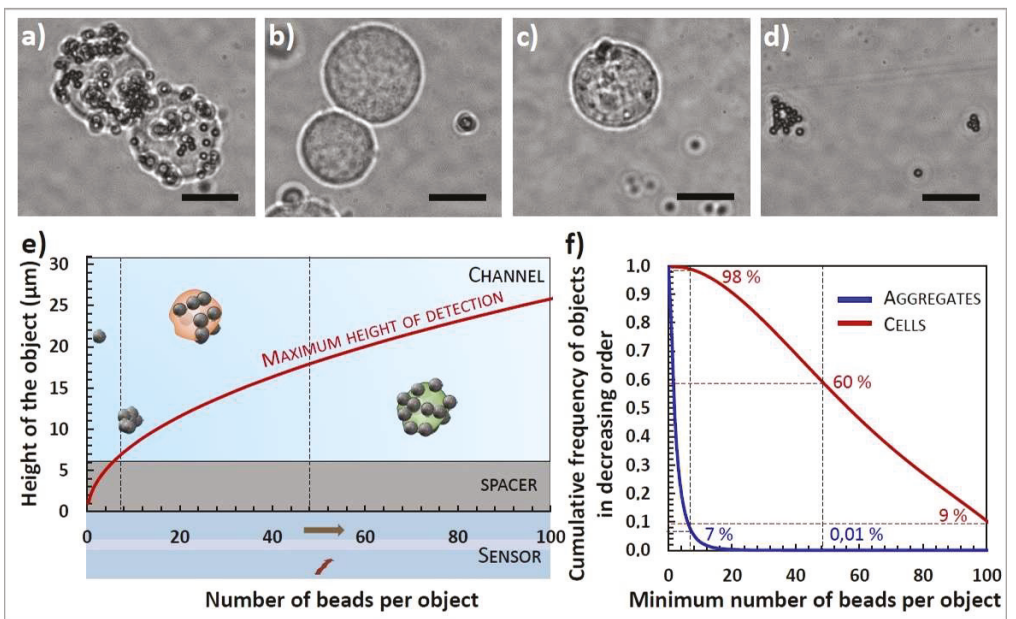

Figure 5. Labeling and aggregation study. Four sample photographs (magnification 100) were taken under optical microscope and illustrate beads repartition. (The scale bars represent $5 \mu \mathrm{m}$.) (a) Group of two NS1 cells labeled with Dynabeads MyOne functionalized with anti-CD138 mAbs. (b) Group of two NS1 cells after two hours-contact with Dynabeads MyOne functionalized with control IpaD-315 mAbs. (c) $\mathrm{CHO}$ cell after two hours-contact with Dynabeads MyOne functionalized with anti-CD138 mAb (d) Dynabeads MyOne functionalized with anti-CD138 mAbs in Phosphate Buffer Saline (PBS). (e) Adapted spacer layer thickness estimation. In this illustration, the detectivity is set to $2.2 \mu \mathrm{T}$. Relation between the number of beads covering an object and the maximum height at which it can be detected. Objects below the red curve are detectable while objects above are not. (f) Corresponding detectable population of cells and aggregates. Graph of the observed cumulative frequency of the number of MPs per NS1 cell and per aggregate in decreasing order. Estimation based on the study of 309 cells in a solution containing $10^{5} \mathrm{NS} 1 / \mathrm{mL}$ and $23 \mu \mathrm{g} / \mathrm{mL}$ anti-CD138 functionalized MPs $/ \mathrm{mL}$ after 2h-contact and of 18,630 aggregates in a suspension containing $23 \mu \mathrm{g} / \mathrm{mL}$ anti-CD138 in PBS.

Knowing the detectivity of the sensor (experimental characterizations are given on Table 2), the maximal distance from the sensor at which an object composed of $\mathrm{N}$ beads can be detected is deduced and plotted in the Figure 5 . Above this distance, all the aggregates containing less than $\mathrm{N}$ MPs are undetectable. While $98 \%$ of NS1 are labeled with more than 7 beads, only $7 \%$ of aggregates are composed of more than 7 beads. This minimum number of beads seems to be a good discrimination factor. The Figure 5e shows that objects of 7 beads are undetectable from $6 \mu \mathrm{m}$ above the sensor.

Adding a separation layer between the sensor and the bottom of the channel eliminates most of the nonspecific signals (from small aggregates and single beads) and improves the discrimination on the number of beads by reducing the importance of the height parameter. Indeed, between 7 and 16 $\mu \mathrm{m}$ height, the signal is divided by approximately 12 only. Without this supplementary layer, objects of small magnetization could still induce large amplitude signals that could be mistaken for labeled cells.

This study leads to the conclusion that the best configuration for our system is the addition of a $6 \mu \mathrm{m}$ thick separation layer between the sensor and the channel. The thickness of the separation layer needs to be optimized for each couple bead/biological target, because it depends strongly on the expected moment per target and thus on the number of antigens expressed by the target. 


\subsection{Performance of the GMR Chip Test}

Seven experiments (See Table 2 and Figure 6a) were realized on six days using 4 similar chips with different sample volumes ranging from 200 to $400 \mu \mathrm{L}$ and the number of events was normalized to a volume of $1 \mathrm{~mL}$. To avoid biased results, samples have been injected in the chip in different orders at each experiment and between two samples the chip was washed with deionized water and dried. For each experiment, the sensitivity and the noise level of the sensor have been measured and the detection thresholds were deduced (given in Table 2). In order to coherently treat data from these seven experiments, only signals above the highest detection threshold $(2.2 \mu \mathrm{T})$ were considered.

Table 2. Experimental conditions of the seven experiments. The threshold of each experiment is defined as the lowest detectable signal (with a signal to noise ratio at 3 ). Results are given as a number of events above $2.2 \mu \mathrm{T}$ detected per milliliter of sample. The average count is given with its standard deviation (SD) for each sample. Control samples are presented at the bottom of the table, separated from positive samples. The highest count in negative samples, in bold, is obtained for anti-CD138 beads in PBS for which the average value added to three standard deviations gives $3.610^{3}$ counts.

\begin{tabular}{|c|c|c|c|c|c|c|c|c|c|}
\hline & & Day 1 & Day 2 & Day 3 & Day 4 & Day 5 & \multicolumn{2}{|c|}{ Day 6} & Summary \\
\hline \multicolumn{2}{|l|}{ Sensor } & \multicolumn{2}{|c|}{ Sensor A } & Sensor B & \multicolumn{2}{|c|}{ Sensor C } & \multicolumn{2}{|c|}{ Sensor D } & Different \\
\hline \multirow{2}{*}{\multicolumn{2}{|c|}{$\begin{array}{l}\text { Separation layer thickness } \\
\text { Channel height }\end{array}$}} & \multicolumn{2}{|c|}{$6.2 \mu \mathrm{m}$} & $6.4 \mu \mathrm{m}$ & \multicolumn{2}{|c|}{$5.7 \mu \mathrm{m}$} & \multicolumn{2}{|c|}{$5.5 \mu \mathrm{m}$} & devices, \\
\hline & & \multicolumn{2}{|c|}{$26.5 \mu \mathrm{m}$} & $27.4 \mu \mathrm{m}$ & \multicolumn{2}{|c|}{$25.3 \mu \mathrm{m}$} & \multicolumn{2}{|c|}{$23.1 \mu \mathrm{m}$} & samples and \\
\hline \multicolumn{2}{|c|}{ Beads batch } & 1 & 2 & 3 & 4 & 5 & \multirow{2}{*}{\multicolumn{2}{|c|}{$\begin{array}{c}5 \\
048\end{array}$}} & conditions. \\
\hline \multirow{2}{*}{\multicolumn{2}{|c|}{$\begin{array}{l}\text { Threshold }(\mu \mathrm{T}) \\
\text { Sample volume }(\mu \mathrm{L})\end{array}$}} & 1.6 & 1.8 & 2.2 & 0.47 & 0.46 & & & Same \\
\hline & & 400 & 200 & 300 & 300 & 300 & 300 & 300 & experimenters \\
\hline Cells & mAbs & \multicolumn{7}{|c|}{ Counts of signals above 2.2 microteslas per milliliter } & Average \pm SD \\
\hline $10^{5} \mathrm{NS} 1$ & anti-CD138 & \multirow[t]{5}{*}{12,123} & \multirow[t]{5}{*}{14,700} & \multirow[t]{5}{*}{10,367} & & & & & $1.2 \times 10^{4} \pm 1.810^{3}$ \\
\hline $310^{4} \mathrm{NS} 1$ & anti-CD138 & & & & 3463 & 5173 & 6180 & 5070 & $5.0 \times 10^{3} \pm 9.710^{2}$ \\
\hline $10^{4} \mathrm{NS} 1$ & anti-CD138 & & & & 2867 & 1223 & 1927 & 1597 & $1.9 \times 10^{3} \pm 6.110^{2}$ \\
\hline $310^{3} \mathrm{NS} 1$ & anti-CD138 & & & & 467 & 630 & 517 & 607 & $5.6 \times 10^{2} \pm 6.610^{1}$ \\
\hline $10^{3}$ NS1 & anti-CD138 & & & & 500 & 977 & 280 & 353 & $5.3 \times 10^{2} \pm 2.710^{2}$ \\
\hline \multirow{3}{*}{$\begin{array}{l}10^{5} \mathrm{NS} 1 \\
10^{5} \mathrm{CHO} \\
\varnothing\end{array}$} & IpaD315 & \multirow[t]{2}{*}{895} & \multirow[t]{2}{*}{60} & \multirow[t]{2}{*}{660} & \multirow[t]{2}{*}{637} & 723 & 313 & 520 & $5.4 \times 10^{2} \pm 2.610^{2}$ \\
\hline & anti-CD138 & & & & & 690 & 380 & 367 & $4.8 \times 10^{2} \pm 1.510^{2}$ \\
\hline & anti-CD138 & 1665 & 375 & 2213 & 310 & & & & $1.1 \times 10^{3} \pm 8.210^{2}$ \\
\hline
\end{tabular}

Between 60 and 2200 counts per milliliter were found in each type of negative control samples in PBS (14 measurements in total). Studying these results allow us to determine a count threshold characterizing the test. The count threshold above which the sample can be considered positive (the detection threshold) is calculated as the average of the values obtained for the negative test having the most counts (thus, the anti-CD138 beads) added to three times their standard deviation. This method concludes to an average non-specific count of $1.110^{3}$ counts per milliliter and a detection count threshold of $3.610^{3}$ counts per milliliter.

Looking at the positive sample results, this threshold corresponds to a concentration between 1 and $310^{4}$ NS1 per milliliter.

\subsection{ELISA Test Sensitivity}

The ELISA test was repeated in three independent experiments in order to compare its detection limit to the one of the GMR sensor. Results are presented in Figure 6b. For this 5 h-test in PBS suspensions, the detection limit (the lowest concentration at which the test can determine positivity of the sample) was found equal to $2.010^{4} \mathrm{NS} 1 / \mathrm{mL} \pm 1.810^{4}$ while the quantification limit (the lowest concentration at which the test can give a correct estimation of the sample concentration) was found at $6.710^{4} \mathrm{NS} 1 / \mathrm{mL} \pm 2.910^{4}$. 


\subsection{Flow Cytometry Results}

The number of NS1 cells was evaluated by flow cytometry using a specific monoclonal antibody against the CD138 surface molecule. The living cells were selected by size and cell granularity and the number of CD138+ cells in each sample was evaluated by comparison with cells incubated with buffer alone. As can be seen in Figure 6c, the evaluation of the number of positive cells for the CD138 marker is possible up to a cell concentration in the sample equal to $310^{3}$ cells $/ \mathrm{mL}$.

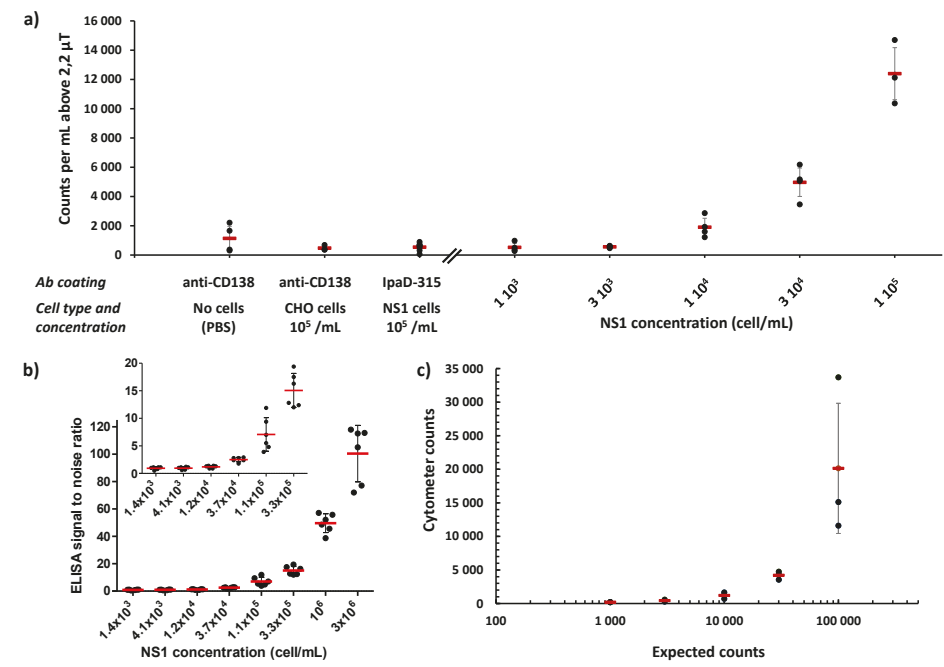

Figure 6. Experimental results of the three tests. All were performed in different days, thus the same samples were not tested with the three techniques. (a) Experimental results of the GMR test. Red dashes represent the mean of the experiments. Error bars represent standard deviations from the experiments. (b) Experimental results of the ELISA tests. Different concentrations of NS1 cells in PBS were detected using the homologous sandwich ELISA involving anti-CD138 mAb as capture and tracer antibody in a $5 \mathrm{~h}$ sequential format. The signal to noise ratio was calculated from the mean of eight measurements of PBS alone. Red dashes represent the mean of the three independent experiments, each performed in duplicate. Error bars represent standard deviations from the three experiments. The insert shows the low concentration part of the curve. (c) Experimental results of flow cytometry presented as counts per milliliter for each concentration. Red dashes represent the mean of the three independent experiments. Error bars represent standard deviations from the three experiments.

\section{Conclusions}

In this article, the different steps of the conception of a magnetoresistive chip cell-counter were detailed. This detection technique has a great potential. The production, use and integrability of GMR sensors are easy and the tool allows for the detection of targets one by one. This test was evaluated regarding several essential qualities of diagnostic tools (sensitivity, specificity, reproducibility and duration) on a biological model, murine myeloma cells immunocaptured by commercial magnetic beads of $1 \mu \mathrm{m}$ in diameter. The reached sensitivity of about $10^{4}$ cells $/ \mathrm{mL}$ is equivalent to that of an ELISA test realized with the same reagents (NS1 cells, mAbs, buffer ...). Our test is simpler to perform than an ELISA test. Indeed, the GMR test can be performed within $2 \mathrm{~h} 30$ ( $2 \mathrm{~h}$ of labeling as assessed by our kinetic study, briefly described in Appendix A and $30 \mathrm{~min} / \mathrm{mL}$ of sample for the test) without any washing steps, while the compared ELISA test requires several washing steps. Data treatment can be done in a few minutes for ELISA test and can be integrated in the acquisition chain and done in real time for the GMR test. One can note that both techniques can benefit from large parallelization of tests. Moreover, the time of the GMR test can be further reduced by increasing the flow rate in the channel. 
The labelling processes strongly depends on the target, the beads and the biological probe and other groups reported times between 30 and $180 \mathrm{~min}[51,53,57]$. This time will have to be optimized on the final system, in the real biological sample.

Flow cytometry, although not optimized to give absolute cell counts, have a sensitivity ten times lower than the GMR test. However, this method is more complicated, with washing steps, causing loss of cells and thus discrepancies in counts. The Figure 7 shows the extrapolated number of cells counted by both techniques in positive samples of $1 \mathrm{~mL}$ as a function of the expected counts. The agreement between the two techniques is remarkably good for all concentrations except at $10^{3} \mathrm{NS} 1 / \mathrm{mL}$. Our technique however, presents the interest that the count of signals can be automated while flow cytometry data treatment requires an expert.

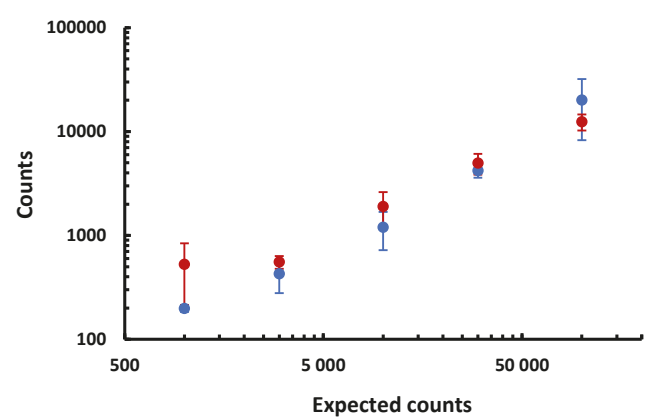

Figure 7. Experimental results of flow cytometry (in blue) compared with experimental results of the GMR test (in red). Mean values and standard deviations are represented.

The relatively high limit of detection of some $10^{4}$ cells $/ \mathrm{mL}$ is due to two main phenomena. First, some specific events are missed. Indeed, some less efficiently labeled cells are flowing high in the channel and cannot be detected specifically. Secondly, the detection count threshold has a high value. This can be explained both by the number of beads aggregates, increasing the average number of non-specific signals and by the variability of experimental parameters, increasing the standard deviation of the number of non-specific signals. These uncertainties rise from the use of 5 distinct batches of functionalized beads for the experiment, the random order in which the samples were passed and the involuntarily fluctuations in channel geometry.

This study shows the importance to take into account the biological parameters (antigen distribution, labeling efficiency, cell survival, matrix effect, etc.) in the test evaluation. The high detection count threshold value demonstrates the crucial importance of having negative controls and to repeat experiments in different conditions several times in order to define correctly performances of such technologies. The development of diagnostic tests are based on these two pillars (physical and biological parameters) and correct definitions of performances of a test should systematically integrate these cross-cutting aspects. Here, the focus was set on a rigorous evaluation of non-specific signals measured by the GMR sensor. The study showed that these non-specific signals were due to the detection of beads aggregates.

To lower the detection threshold without complicating the device, the challenge is to diminish drastically the number and the sizes of the MP aggregates. As a matter of fact, decreasing the number of beads in aggregates would enable great changes in the chip design. The separation layer, added to reduce the impact of non-specific events, could be thinned and thus less efficiently labeled cells would be easier to detect. A better understanding of these aggregation phenomena and development of solutions to reduce the number of these non-specific events will help to reach a better reproducibility and sensitivity. 
The elimination of aggregates can be performed by microfluidics sorting techniques relying on hydrodynamic or magnetodynamic forces [61-65] but this would necessarily waste a certain amount of expensive mAbs-coated beads. Another way to deal with these beads suspensions instabilities would be to address directly the cause by a better design of the magnetic beads, such as adding a PEG coating [66].

The real solution may lie in designing magnetic beads tuned especially for this application and thus, to continue the development of this diagnosis tool, the natural next step should be to add chemistry as a third project pillar.

Author Contributions: Conceptualization, M.G., F.-D.D., C.F., C.F.-T., S.S. and G.J.-L.; Data curation, M.G. and C.F.-T.; Formal analysis, M.G., C.F.-T. and G.J.-L.; Funding acquisition, E.E., S.S. and G.J.-L.; Investigation, M.G., F.-D.D., A.W. and C.F.-T.; Methodology, F.-D.D., C.F., C.F.-T., S.S. and G.J.-L.; Project administration, G.J.-L.; Resources, G.C., M.P. and E.P.; Software, P.B., M.T. and C.F.; Supervision, C.F., C.F.-T., S.S. and G.J.-L.; Validation, C.F.-T. and G.J.-L.; Visualization, M.G.; Writing—original draft, M.G.; Writing—review \& editing, F.-D.D., A.W., C.F., C.F.-T., S.S. and G.J.-L.

Funding: This research was funded by the Direction Générale de l'Armement, the Ile de France region and its DIM-ELICIT program and the CEA transverse programs "Techno-Santé" and "PTC Instrumentation et Détection".

Acknowledgments: We are greatly indebted to Bakhos Jneid and Karine Devilliers who produced the recombinant IpaD protein and the monoclonal antibody IpaD315. We would like to thank Gérald Le Goff and Dominique Duet for their regular help with technical issues. We are grateful to Olivier Limousin, Antoine Pallandre, Anne-Marie Haghiri-Gosnet, Auélie Solignac and Hervé Volland for fruitful scientific exchanges.

Conflicts of Interest: The authors declare no conflict of interest.

\section{Abbreviations}

The following abbreviations are used in this manuscript:

BSA Bovine Serum Albumine

CHO Chineese Hamster Ovary cells

ELISA Enzyme-Linked ImmunoSorbent Assay

GMR Giant Magneto-Resistance

$\mathrm{mAb}$ Monoclonal antibody

MP Magnetic Particle

PBS Phosphate buffer saline

PDMS Polydimethylsiloxane

\section{Appendix A. Magnetic Particles Aggregation Study}
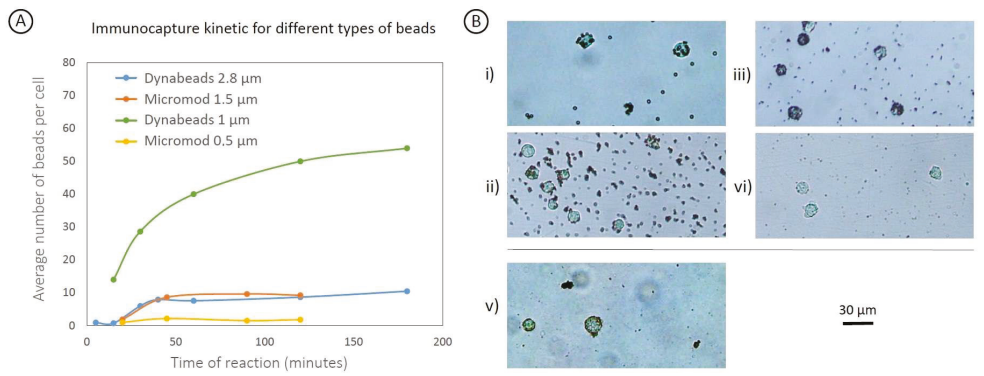

Figure A1. (A) Kinetic of NS1 labeling for 4 types of microbeads. (B) 20 magnification photographs of NS1 cells incubated $2 \mathrm{~h}$ with: (i) Dynabeads $2.8 \mu \mathrm{m}$ (ii) Micromod $1.5 \mu \mathrm{m}$ (iii) Dynabeads $1 \mu \mathrm{m}$ (iv) Micromod $0.5 \mu \mathrm{m}(\mathrm{v})$ Adembeads $200 \mathrm{~nm}$.

mAbs-functionalized $200 \mathrm{~nm}$ carboxyl-Adembeads (Ademtech ${ }^{\circledR}$ ), $500 \mathrm{~nm}$ streptavidin nanomag ${ }^{\circledR}-\mathrm{D}$ (Micromod), $1 \mu \mathrm{m}$ Dynabeads ${ }^{\mathrm{TM}} \mathrm{MyOne}^{\mathrm{TM}}$ Streptavidin $\mathrm{T} 1$ (Invitrogen $^{\mathrm{TM}}$ ), $1.5 \mu \mathrm{m}$ streptavidin sicastar $\left(\mathrm{R}-\mathrm{M}\right.$ (Micromod) and $2.8 \mu \mathrm{m}$ Dynabeads ${ }^{\mathrm{TM}} \mathrm{M}-280$ Tosylactivated (Invitrogen ${ }^{\mathrm{TM}}$ ) 
suspensions were analyzed in terms of aggregate distribution (data not shown). Then, cell immunocapture kinetic studies were realized with an optical microscope. Data from $200 \mathrm{~nm}$ beads were hardly exploited but it was clear that beads aggregated considerably while no excessive labeling was visible at cell surface (Figure $\mathrm{A} 1 \mathrm{~B}(\mathrm{v})$ ). The other 4 types of MPs gave quantified results presented on Figure A1 as plots of average number of beads per cell against time. The $500 \mathrm{~nm}$-diameter Micromod beads labelled poorly the cells after $2 \mathrm{~h}$. $1.5 \mu \mathrm{m}$ diameter Micromod beads labelled the cells correctly but aggregates were far too numerous for those beads to be of any interest. The best options were the two kinds of Dynabeads ${ }^{\mathrm{TM}}$. The estimation of number and size of aggregates for these beads showed that $1 \mu \mathrm{m}$ beads seemed to form labeled cells more distinguishable from beads aggregates than the 2.8 ones. However, to confirm that, there were attempts to decrease the number of aggregates for $2.8 \mu \mathrm{m}$ beads as they reached the maximum cell labeling quicker. They were sonicated for 30 $\mathrm{s}$ to 1 minute for 1 to 3 times before use with no conclusive results. Then, they were vortexed and sonicated alternatively 3 times with cycles of 2 and 1 min respectively. Finally they were added to several detergent alone or in combinations at $0.1 \%$ each (SDS (sodium dodecyl sulfate), tween 20 , triton X-100, CHAPS (3-[(3-cholamidopropyl)dimethylammonio]-1-propanesulfonate)) either in PBS or in deionized water before being submitted to the 3 cycles of sonication-vortices described above. While most of these attempts remained unsuccessful, the suspension of beads in deionized water containing all detergents and the suspension in deionized water containing SDS and tween 20 showed significant improvements: the percentage of single beads improved from $66 \%$ in the suspension in PBS without detergent to $90 \%$ and $84 \%$ respectively. Once this observation was done, the cell survival was evaluated in the presence of different detergent concentrations ranging from $0.01 \%$ to $1 \%$. All cells were lysed after $2 \mathrm{~h}$ contact with all these detergent concentrations. Dynabeads ${ }^{\mathrm{TM}}$ of $1 \mu \mathrm{m}$ were thus the optimum and detergent use was abandoned. Sonication and vortex did not improve their aggregation.

\section{References}

1. Bialy, L.; Mlynarczuk-Bialy, I. Advances in Biomedical Research-Selected Topics; Wydawnictwo Naukowe TYGIEL sp. z o.o.: Lublin, Poland, 2018.

2. Cohen, S.J.; Punt C.J.; Iannotti N.; Saidman B.H.; Sabbath K.D.; Gabrail N.Y.; Picus J.; Morse M.; Mitchell E.; Miller M.C.; et al. Relationship of Circulating Tumor Cells to Tumor Response, Progression-Free Survival, and Overall Survival in Patients With Metastatic Colorectal Cancer. J. Clin. Oncol. 2008, 26, 3213-3221. [CrossRef] [PubMed]

3. Giuliano, M.; Giordano, A.; Jackson, S.; Hess, K.R.; De Giorgi, U.; Mego, M.; Handy, B.C.; Ueno, N.T.; Alvarez, R.H.; De Laurentiis, M.; et al. Circulating tumor cells as prognostic and predictive markers in metastatic breast cancer patients receiving first-line systemic treatment. Breast Cancer Res. 2011, 13, 1-9. [CrossRef] [PubMed]

4. Sinha, M.; Jupe, J.; Mack, H.; Coleman, T.P.; Lawrence, S.M.; Fraley, S.I. Emerging Technologies for Molecular Diagnosis of Sepsis. Clin. Microbiol. Rev. 2018, 31, e00089-17. [CrossRef] [PubMed]

5. Fitzmaurice, C.; Allen, C.; Barber, R.M.; Barregard, L.;, Bhutta, Z.A.; Brenner, H.; Dicker, D.J.; Chimed-Orchir, O.; Dandona, R.; Dandona, L.; et al. Global, Regional, and National Cancer Incidence, Mortality, Years of Life Lost, Years LivedWith Disability, and Disability-Adjusted Life-years for 32 Cancer Groups, 1990 to 2015 A Systematic Analysis for the Global Burden of Disease Study. JAMA Oncol. 2017, 3, 524-548.

6. Fulwyler, M.J. Electronic Separation of Biological Cells by Volume. Science 1965, 150, 910-911. [CrossRef]

7. Aebisher, D.; Bartusik, D.; Tabarkiewicz, J. Laser flow cytometry as a tool for the advancement of clinical medicine. Biomed. Pharmacother. 2017, 85, 434-443. [CrossRef] [PubMed]

8. Brown, M.; Wittwer, C. Flow Cytometry: Principles and Clinical Applications in Hematology. Clin. Chem. 2000, 46, 1221-1229.

9. Bakke, A.C. Clinical Applications of Flow Cytometry. Lab. Med. 2000, 31, 97-104. [CrossRef]

10. Wood, B.L. Principles of Minimal Residual Disease Detection for Hematopoietic Neoplasms by Flow Cytometry. Cytom. Part B 2016, 90, 47-53. [CrossRef]

11. Chernecky, C.C.; Berger, B.J. Laboratory Tests and Diagnostic Procedures; Elsevier Health Sciences: Saint Louis, MO, USA, 2012. 
12. Féraudet-Tarisse, C.; Vaisanen-Tunkelrott, M.L.; Moreau, K.; Lamourette, P.; Créminon, C.; Volland, H. Pathogen-free screening of bacteria-specific hybridomas for selecting high-quality monoclonal antibodies against pathogen bacteria as illustrated for Legionella pneumophila. J. Immunol. Methods 2013, 391, 81-94. [CrossRef]

13. Gan, S.D.; Patel, K.R. Enzyme Immunoassay and Enzyme-Linked Immunosorbent Assay. J. Investig. Dermatol. 2013, 133, e12. [CrossRef]

14. Piyasena, M.E.; Graves, S.W. The intersection of flow cytometry with microfluidics and microfabrication. Lab Chip 2014, 14, 1044-1059. [CrossRef] [PubMed]

15. Gong, Y.; Fan, N.; Yang, X.; Peng, B.; Jiang, H. New advances in microfluidic flow cytometry. Electrophoresis 2018, 40, 1212-1229. [CrossRef] [PubMed]

16. Mendez-Gonzalez, D.; Lopez-Cabarcos, E.; Rubio-Retama, J.; Laurenti, M. Sensors and bioassays powered by upconverting materials. Adv. Colloid Interface Sci. 2017, 249, 66-87. [CrossRef] [PubMed]

17. MagArray. Available online: http://www.magarray.com/ (accessed on 5 February 2019).

18. Zepto. Available online: http:/ / www.zeptolife.com/(accessed on 5 February 2019).

19. Magnomics. Available online: http://www.magnomics.pt/(accessed on 5 February 2019).

20. Krishna, V.D.; Wu, K.; Perez, A.M.; Wang, J.P. Giant Magnetoresistance-based Biosensor for Detection of Influenza A Virus. Front. Microbiol. 2016, 7, 400. [CrossRef]

21. Ferguson, B.S.; Buchsbaum, S.F.; Wu, T.T.; Hsieh, K.; Xiao, Y.; Sun, R.; Soh, R.T. Genetic analysis of H1N1 influenza virus from throat swab samples in a microfluidic system for point-of-care diagnostics. J. Am. Chem. Soc. 2011, 133, 9129-9135. [CrossRef]

22. Pihíková, D.; Kasák, P.; Tkac, J. Glycoprofiling of cancer biomarkers: Label-free electrochemical lectin-based biosensors. Open Chem. 2015, 13, 636-655. [CrossRef]

23. Serrate, D.; De Teresa, J.M.; Marquina, C.; Marzo, J.; Saurel, D.; Cardoso, F.A.; Cardoso, S.; Freitas, P.P.; Ibarra, M.R. Quantitative biomolecular sensing station based on magnetoresistive patterned arrays. Biosens. Bioelectron. 2012, 35, 206-212. [CrossRef]

24. Fermon, C.; Van de Voorde, M. Nanomagnetism, Applications and Perspectives; Wiley: Weinheim, Germany; 2017.

25. Lin, G.; Makarov, D.; Schmidt, O.G. Magnetic sensing platform technologies for biomedical applications. Lab Chip 2017. [CrossRef]

26. Wang, T.; Yang, Z.; Lei, C.; Lei, J.; Zhou, Y. An integratedgiantmagnetoimpedancebiosensorfordetection of biomarker. Biosens. Bioelectron. 2014, 58, 338-344. [CrossRef]

27. Boyle, D.S.; Hawkins, K.R.; Steele, M.S.; Singhal, M.; Cheng, X. Emerging technologies for point-of-care CD4 T-lymphocyte counting. Trends Biotechnol. 2012, 30, 45-54. [CrossRef] [PubMed]

28. Barroso, T.G.; Martins, R.C.; Fernandes, E.; Cardoso, S.; Rivas, J.; Freitas, P.P. Detection of BCG bacteria using a magnetoresistive biosensor: A step towards a fully electronic platform for tuberculosis point-of-care detection. Biosens. Bioelectron. 2018, 100, 259-265. [CrossRef] [PubMed]

29. Lange, J.; Kötitz, R.; Haller, A.; Trahms, L.; Semmler, W.; Weitschies, W. Magnetorelaxometry-A new binding specific detection method based on magnetic nanoparticles. J. Magn. Magn. Mater. 2002, 252, 381-383. [CrossRef]

30. Yang, T.Q.; Abe, M.; Horiguchi, K.; Enpuku, K. Detection of magnetic nanoparticles with ac susceptibility measurement. Phys. C 2004, 412-414, 1496-1500. [CrossRef]

31. Ludwig, F.; Heima, E.; Mä useleina, S.; Eberbeckb, D.; Schilling, M. Magnetorelaxometry of magnetic nanoparticles with fluxgate magnetometers for the analysis of biological targets. J. Magn. Magn. Mater. 2005, 293, 690-695. [CrossRef]

32. Denmark, D.J.; Bustos-Perez, X.; Swain, A.; Phan, M.H.; Mohapatra, S.; Mohapatra, S.S. Readiness of Magnetic Nanobiosensors for Point-of-Care Commercialization. J. Electron. Mater. 2019, 48, 4749-4761. [CrossRef]

33. Guo, L.; Yang, Z.; Zhi, S.; Feng, Z.; Lei, C.; Zhou, Y.. A sensitive and innovative detection method for rapid C-reactive proteins analysis based on a micro-fluxgate sensor system. PLoS ONE 2018, 13, e0194631. [CrossRef]

34. Pekas, N.; Porter, M.D. Giant magnetoresistance monitoring of magnetic picodroplets in an integrated microfluidic system. Appl. Phys. Lett. 2004, 85, 4783-4785. [CrossRef] 
35. Loureiro, J.; Ferreira, R.; Cardoso, S.; Freitas, P.P.; Germano, J.; Fermon, C.; Arrias, G.; Pannetier-Lecoeur, M.; Rivadulla, F.; Rivas, J. Toward a magnetoresistive chip cytometer: Integrated detection of magnetic beads flowing at cm/s velocities in microfluidic channels. Appl. Phys. Lett. 2009, 95, 034104. [CrossRef]

36. Loureiro, J.; Andrade, P.Z.; Cardoso, S.; da Silva, C.L.; Cabral, J.M.; Freitas, P.P. Magnetoresistive chip cytometer. Lab Chip 2011, 11, 2255-2261. [CrossRef]

37. Walt, D.R. Optical methods for single molecule detection and analysis. Anal. Chem. 2013, 83, 1258-1263. [CrossRef] [PubMed]

38. Helou, M.; Reisbeck, M.; Tedde, S.F.; Richter, L.; Bär, L.; Bosch, J.J.; Stauber, R.H.; Quandt, E.; Hayden, O. Time-of-flight magnetic flow cytometry in whole blood with integrated sample preparation. Lab Chip 2013, 13, 1035-1038. [CrossRef] [PubMed]

39. Muluneh, M.; Issadore, D. Microchip-based detection of magnetically labeled cancer biomarkers. Adv. Drug Deliv. Rev. 2014, 66, 101-109. [CrossRef] [PubMed]

40. Murali, P.; Niknejad, A.M.; Boser, B.E. CMOS Microflow Cytometer for Magnetic Label Detection and Classification. IEEE J. Solid-State Circuits 2017, 52, 543-555. [CrossRef]

41. Giouroudi, I.; Kokkinis, G. Recent Advances in Magnetic Microfluidic Biosensors. Nanomaterials 2017, 7, 171. [CrossRef] [PubMed]

42. Fodil, K.; Denoual, M.; Dolabdjian, C.; Treizebre, A.; Senez, V. In-flow detection of ultra-small magnetic particles by an integrated giant magnetic impedance sensor. Appl. Phys. Lett. 2016, 108, 173701. [CrossRef]

43. García-Arribas, A.; Martínez, F.; Fernández, E.; Ozaeta, I.; Kurlyandskaya, G.V.; Svalov, A.V.; Berganzo, J.; Barandiaran, J.M. GMI detection of magnetic-particle concentration in continuous flow. Sens. Actuators A 2011, 172, 103-108. [CrossRef]

44. Blanc-Béguin, F.; Nabily, S.; Gieraltowski, J.; Turzo, A.; Querellou, S.; Salaun, P. Cytotoxicity and GMI bio-sensor detection of maghemite nanoparticles internalized into cells. J. Magn. Magn. Mater. 2009, 321, 192-197. [CrossRef]

45. Köhler, G.; Milstein, C. Continuous cultures of fused cells secreting antibody of predefined specificity. Nature 1975, 256, 495-497. [CrossRef]

46. Delahaut, P. Immunisation-Choice of host, adjuvants and boosting schedules with emphasis on polyclonal antibody production. Methods 2017, 116, 4-11. [CrossRef]

47. Giouroudi, I.; Hristoforou, E. Perspective: Magnetoresistive sensors for biomedicine. J. Appl. Phys. 2018, 124, 030902. [CrossRef]

48. Lenz, J.E. A Review of Magnetic Sensors. Proc. IEEE 1990, 78, 973-989. [CrossRef]

49. Nabaei, V.; Chandrawati, R.; Heidari, H. Magnetic biosensors: Modelling and simulation. Biosens. Bioelectron. 2018, 103, 69-86. [CrossRef]

50. Pannetier-Lecoeur, M. Superconducting-Magnetoresistive Sensor: Reaching the Femtotesla at 77 K; Habilitation à Diriger des Recherches en Physique, Condensed Matter, Université Pierre et Marie Curie-Paris VI: Paris, France, 2010.

51. Issadore, D.; Chung, J.; Shao, H.; Liong, M.; Ghazani, A.A.; Castro, C.M.; Weissleder, R.; Lee, H. Ultrasensitive clinical enumeration of rare cells ex vivo using a micro-Hall detector. Sci. Transl. Med. 2012, 4, 141 ra92. [CrossRef] [PubMed]

52. Issadore, D.; Chung, H.J.; Chung, J.; Budin, G.; Weissleder, R.; Lee, H. $\mu$ Hall chip for sensitive detection of bacteria. Adv. Healthc. Mater. 2013, 2, 1224-1228. [CrossRef] [PubMed]

53. Chícharo, A.; Martins, M.; Barnsley, L.C.; Taouallah, A.; Fernandes, J.; Silva, B.F.B.; Cardoso, S.; Diéguez, L.; Espiña, B.; Freitas, P.P. Enhanced magnetic microcytometer with 3D flow focusing for cell enumeration. Lab Chip 2018, 18, 2593-2603. [CrossRef] [PubMed]

54. Reisbeck, M.; Richter, L.; Helou, M.J.; Arlinghaus, S.; Anton, B.; van Dommelen, I.; Nitzsche, M.; Baßler, M.; Kappes, B.; Friedrich, O.; et al. Hybrid integration of scalable mechanical and magnetophoretic focusing for magnetic flow cytometry. Biosens. Bioelectron. 2018, 109, 98-108. [CrossRef] [PubMed]

55. Duffy, D.C.; McDonald, J.C.; Schueller, O.J.A.; Whitesides, G.M. Rapid Prototyping of Microfluidic Systems in Poly(dimethylsiloxane). Ana. Chem. 1998, 70, 4974-4984. [CrossRef] [PubMed]

56. Sierocki, R.; Jneid, B.; Rouaix, A.; Plaisance, M.; Féraudet-Tarisse, C.; Maillère, B.; Nozach, H; Simon, S. An antibody targeting type III secretion system induces broad protection against Salmonella and Shigella infections. Unpublished manuscript, 2019. 
57. Reisbeck, M.; Helou, M.J.; Richter, L.; Kappes, B.; Friedrich, O.; Hayden, O. Magnetic fingerprints of rolling cells for quantitative flow cytometry in whole blood. Sci. Rep. 2016, 6, 32838. [CrossRef] [PubMed]

58. Lee, C.P.; Lai, M.F.; Huang, H.T.; Lin, C.W.; Wei, Z.H. Wheatstone bridge giant-magnetoresistance based cell counter. Biosens. Bioelectron. 2014, 57, 48-53. [CrossRef] [PubMed]

59. Pannetier, M.; Fermon, C.; Le Goff, G.; Simola, J.; Kerr, E. Low noise magnetoresistive sensors for current measurement and compasses. J. Magn. Magn Mater. 2007, 316, 246-248. [CrossRef]

60. Li, G.; Wang, S.X.; Sun, S. Model and Experiment of Detecting Multiple Magnetic Nanoparticles as Biomolecular Labels by Spin Valve Sensors. IEEE Trans. Magn. 2004, 40, 3000-3002. [CrossRef]

61. Huang, L.R.; Cox, E.C.; Austin, R.H.; Sturm, J.C. Continuous particle separation through deterministic lateral displacement. Science 2004, 304, 987-990. [CrossRef] [PubMed]

62. El Hasnia, A.; Göbbels, K.; Thiebes, A.L.; Bräunig, P.; Mokwa, W.; Schnakenberg, U. Focusing and sorting of particles in spiral microfluidic channels. Procedia Eng. 2011, 25, 1197-1200. [CrossRef]

63. Salafi, T.; Zeming, K.K.; Zhang, Y. Advancements in microfluidics for nanoparticle separation. Lab Chip 2017, 17, 11-33. [CrossRef]

64. McGrath, J.; Jimenez, M.; Bridle, H. Deterministic lateral displacement for particle separation: A review. Lab Chip 2014, 14, 4139-4158. [CrossRef] [PubMed]

65. Pamme, N.; Manz, A. On-Chip Free-Flow Magnetophoresis: Continuous Flow Separation of Magnetic Particles and Agglomerates. Anal. Chem. 2004, 76, 7250-7256. [CrossRef]

66. Illés, E.; Tombácz, E.; Szekeres, M.; Tóth, I.Y.; Szabó, A.; Iván, B. Novel carboxylated PEG-coating on magnetite nanoparticles designed for biomedical applications. J. Magn. Magn. Mater. 2015, 380, 132-139. [CrossRef]

(c) 2019 by the authors. Licensee MDPI, Basel, Switzerland. This article is an open access article distributed under the terms and conditions of the Creative Commons Attribution (CC BY) license (http:/ / creativecommons.org/licenses/by/4.0/). 
Article

\title{
Towards CMOS Integrated Microfluidics Using Dielectrophoretic Immobilization ${ }^{\dagger}$
}

\author{
Honeyeh Matbaechi Ettehad ${ }^{1, *}$, Rahul Kumar Yadav ${ }^{1}$, Subhajit Guha ${ }^{1}$ and Christian Wenger ${ }^{1,2}$ \\ 1 IHP-Leibniz-Institut für innovative Mikroelektronik, Im Technologiepark 25, 15236 Frankfurt/Oder, \\ Germany; yadav@ihp-microelectronics.com (R.K.Y.); guha.ihp2608@gmail.com (S.G.); \\ wenger@ihp-microelectronics.com (C.W.) \\ 2 Brandenburg Medical School Theodor Fontane, 16816 Neuruppin, Germany \\ * Correspondence: matbaechi@ihp-microelectronics.com; Tel.: +49-335-5625 (ext. 663) \\ $+\quad$ This paper is an expanded version of "Matbaechi Ettehad, H.; Guha, S.; Wenger, C. Simulation of CMOS \\ Compatible Sensor Structures for Dielectrophoretic Biomolecule Immobilization. In Proceedings of the \\ COMSOL_Bioscience and Bioengineering COMSOL, Rotterdam, Netherlands, 19 October 2017."
}

Received: 30 March 2019; Accepted: 30 May 2019; Published: 5 June 2019

\begin{abstract}
Dielectrophoresis (DEP) is a nondestructive and noninvasive method which is favorable for point-of-care medical diagnostic tests. This technique exhibits prominent relevance in a wide range of medical applications wherein the miniaturized platform for manipulation (immobilization, separation or rotation), and detection of biological particles (cells or molecules) can be conducted. DEP can be performed using advanced planar technologies, such as complementary metal-oxide-semiconductor (CMOS) through interdigitated capacitive biosensors. The dielectrophoretically immobilization of micron and submicron size particles using interdigitated electrode (IDE) arrays is studied by finite element simulations. The CMOS compatible IDEs have been placed into the silicon microfluidic channel. A rigorous study of the DEP force actuation, the IDE's geometrical structure, and the fluid dynamics are crucial for enabling the complete platform for CMOS integrated microfluidics and detection of micron and submicron-sized particle ranges. The design of the IDEs is performed by robust finite element analyses to avoid time-consuming and costly fabrication processes. To analyze the preliminary microfluidic test vehicle, simulations were first performed with non-biological particles. To produce DEP force, an AC field in the range of 1 to $5 \mathrm{~V}$ (peak-to-peak) is applied to the IDE. The impact of the effective external and internal properties, such as actuating DEP frequency and voltage, fluid flow velocity, and IDE's geometrical parameters are investigated. The IDE based system will be used to immobilize and sense particles simultaneously while flowing through the microfluidic channel. The sensed particles will be detected using the capacitive sensing feature of the biosensor. The sensing and detecting of the particles are not in the scope of this paper and will be described in details elsewhere. However, to provide a complete overview of this system, the working principles of the sensor, the readout detection circuit, and the integration process of the silicon microfluidic channel are briefly discussed.
\end{abstract}

Keywords: biomolecules; microfluidic; dielectrophoretic immobilization; CMOS biosensor; lab-on-chip

\section{Introduction}

Dielectrophoresis (DEP) is the change in the dielectric behavior of the particles placed in a non-uniform AC electric field [1]. When particles are subjected to this AC field, dipole moments are induced in the particles. The particle movement is dependent on the polarizability of the particle relative to its suspending medium. The DEP force is strongly dependent on the particle diameters, E-Field vectors, frequencies, and dielectric properties of the particles and their surrounding medium. The Clausius Mosotti (CM) factor depends on the properties of the particle and the medium, as well as 
the frequency of the exciting field [2]. The permittivity of the particles is the dominating parameter affecting the $\mathrm{CM}$ factor. The frequency of the DEP field is unique for every particle. The $\mathrm{CM}$ factor defines the sign of the DEP force. Positive CM values lead to a positive DEP (pDEP) force on the particles, pushing them towards the denser E-Field regions. This occurs when the polarizability of the particle is larger than the polarizability of the fluid. Negative CM values result in negative DEP (nDEP) and drive the particles away from the dense E-Field regions [3,4]. DEP techniques are widely used in microfluidic biochips, so-called lab-on-a-chip (LOC) devices [4-7]. Based on the size and dielectric properties of the particles, DEP can be applied for manipulation of the particles. In this work, positive DEP is used to immobilize particles on the electrodes. By combining the capabilities of complementary metal-oxide-semiconductor (CMOS) based LOC platforms with sensor elements, also called biosensors, the microsystems enable fast diagnostic results [8-11]. The interdigitated biosensor has been previously used to immobilize proteins [12], polystyrene beads, and biological entities [5] as well as detecting organic fluid's dielectric constants [13], suspended biological cells [14], and creatinine molecules [15]. Over recent decades, many investigations have been performed on the detection of bioparticles with CMOS integrated microfluidic systems [7,13,16-21]. Among these investigations, some of the systems were benefited from the sensing and detection on the same BiCMOS integrated platforms. However, using a single sensor for dielectrophoretic manipulation as well as sensing/detecting accompanied by microelectronics circuitry for readout on one single chip has not yet been explored. Table 1 presents a comparison of the reported techniques using both DEP and without DEP on a CMOS platform for biological applications.

Table 1. Comparison of complementary metal-oxide-semiconductor (CMOS) integrated lab-on-chip systems.

\begin{tabular}{|c|c|c|c|c|c|}
\hline Ref. & Particle & Aim & $\begin{array}{c}\text { Particle Manipulation } \\
\text { Platform }\end{array}$ & $\begin{array}{c}\text { Particle } \\
\text { Detecting }\end{array}$ & $\begin{array}{l}\text { Readout } \\
\text { Circuitry }\end{array}$ \\
\hline$[7]$ & $\begin{array}{l}\text { Auto fluorescent protein } \\
\text { R-phycoerythrin } \\
\text { (RPE)/fluorescently } \\
\text { labeled IgG Antibody }\end{array}$ & immobilization & $\begin{array}{c}\text { more than } 100 \mathrm{~K} \\
\text { cylindrical } \\
\text { sub-microelectrodes }\end{array}$ & $\begin{array}{l}\text { monitoring } \\
\text { fluorescence } \\
\text { intensity }\end{array}$ & $\begin{array}{l}\text { potential for } \\
\text { on-chip }\end{array}$ \\
\hline [16] & Living cells $20-30 \mu \mathrm{m}$ & immobilization & $\begin{array}{l}\text { 102,400 actuation } \\
\text { electrodes }\end{array}$ & optical sensor & on-chip \\
\hline [17] & $\begin{array}{l}\text { Micro-nano particles } \\
500 \mathrm{~nm}-10 \mu \mathrm{m}\end{array}$ & separation & L-shaped electrode & $\mathrm{IDE}^{1}$ & off-chip \\
\hline$[18]$ & Yeast cells & immobilization & octa-pole electrode & $\begin{array}{l}\text { trans-impedance } \\
\text { amplifier }\end{array}$ & off-chip \\
\hline [19] & $\begin{array}{l}\text { Fat and calcium in the } \\
\text { blood }\end{array}$ & $\begin{array}{c}\text { plaque } \\
\text { characterization in } \\
\text { arteries }\end{array}$ & - & $\mathrm{IDE}^{1}$ & $\begin{array}{l}\text { potential for } \\
\text { on-chip }\end{array}$ \\
\hline [20] & Yeast cells & $\begin{array}{l}\text { detecting cell } \\
\text { concentration }\end{array}$ & - & $\mathrm{IDE}^{1}$ & on-chip \\
\hline [21] & $\begin{array}{l}\text { A mixture of } \\
\text { glycerol/water and } \\
\text { glycerol/alcohol }\end{array}$ & $\begin{array}{l}\text { detecting the } \\
\text { variation of fluid's } \\
\text { relative viscosity }\end{array}$ & - & $\mathrm{IDE}^{1}$ & on-chip \\
\hline [22] & Yeast cells & $\begin{array}{c}\text { particle } \\
\text { counting/single } \\
\text { particle sensing }\end{array}$ & - & $\mathrm{IDE}^{1}$ & on-chip \\
\hline
\end{tabular}

Originally, Otto et al. [7] described the use of DEP as a simple technique to immobilize the protein particles on conductive surfaces of a CMOS device. These surfaces could be small active regions of micrometer size. Moreover, antibodies were immobilized on the conductive surfaces permanently using DEP. Although this device presented the potential for integration with the microelectronics circuitry, the circuits for data acquisition and processing was omitted. Manaresi et al. [16] developed a LOC architecture based on a two-dimensional array of microsites on a CMOS microsystem for manipulation and detection of individual cells on a standard CMOS microsystem. The $8 \times 8 \mathrm{~mm}^{2}$ chip 
included 102,400 actuation electrodes, embedded memory for electrode programming, and an optical sensor. Although manipulation and detection of cells occurred in a parallel manner, generating the electric field to create DEP cage and cell detection was obtained through different units. To create a DEP cage, counter electrodes surrounded by in-phase electrodes were used. The optical sensor detected the presence of the trapped cells. Miled et al. [17] proposed a microfluidic structure containing 64 electrodes for the manipulation of cells. These electrodes were capable of moving, separating, and rotating the cells. Additionally, an interdigitated electrode was embedded in the microfluidic channel. This electrode was responsible for detecting the microfluidic channel capacitance alteration and was connected to a CMOS chip for data acquisition. A CMOS chip monitored the DEP dependent variables (e.g., frequency and voltage) to evaluate the cross-over frequencies, which can be characteristic for each cell. However, this system does not benefit from simultaneous cell manipulation and detection. Another CMOS based LOC platform was developed in the work of Park et al. [18] for trapping, rotating, and detecting cells and microorganism using DEP. The proposed system used octa-pola electrodes to trap and rotate the particles. Simultaneously, these particles were detected by in-situ impedance monitoring on the same chip. This single chip platform included an on-chip Trans-Impedance Amplifier wire bonded to a printed circuit board (PCB) with external circuits to readout the change in impedance. To avoid the contact of aqueous biological media with the electronics of the chip, polydimethylsiloxane (PDMS) wells were fabricated on the chip.

Guha et al. [19-22] has extensively worked on developing single BiCMOS LOC platforms for biological cell sensing and detection using different techniques. In all of these works, the sensing principle of this sensor is based on the relative permittivity change of the material-under-test (MUT). Variation in biological cell resulted in the fringing field capacity change of the sensor, which caused capacitance variations to be detected by an associated silicon high-frequency readout circuit. By applying electric potential to the electrodes, a non-uniform electric field between the adjacent fingers is generated. This method is used to detect the dielectric permittivity of the MUT. Guha et al. [19] presented a CMOS based high-frequency sensor with the capability of distinguishing the blood sample with fat and calcium from the normal blood sample. The sensor is placed at the top and inside the wall of the catheter and exposed to the blood. In other work [20], he proposed the integration of the silicon microfluidic channel with a CMOS sensor circuit for cytometric applications to detect the cells concentrations using dielectric spectroscopy. In this method, the interdigitated electrode (IDE) arrays were placed on top of the microfluidic channel. As a result of IDE excitation, the fringing electric fields penetrate into the fluid flowing through the channel. They also investigated the variation of the relative viscosity in an aqueous solution [21] using radio frequency (RF) CMOS chem-bio sensor. The fringing field between the adjacent fingers is utilized to detect the dielectric permittivity of the MUT. Furthermore, he developed a self-calibrating highly sensitive dynamic IDE sensor in a BiCMOS based PDMS microfluidic platform that can be applied for particle counting and single particle sensing in a fluidic system [22]. The steady flow of the particles suspended in the fluid results in capacitive pulses from the sensor that is embedded in the oscillator. Eventually, these pulses translate to frequency modulation using an integrated phase-locked loop demodulator.

In this work, an IDE sensor is proposed and designed to immobilize the particles using the DEP technique. This IDE platform can be integrated with circuits and microfluidic channel by CMOS process line of IHP-Leibniz-Institut für innovative Mikroelektronik, for simultaneous immobilization, sensing, and detection of particles. The geometry of the IDEs has been adapted from the previous works done at IHP $[14,15]$. This work aims to enable the design of an IDE with the capability to immobilize micron and nano-sized particles. By placing an AC voltage source to the electrodes, a non-uniform electric field is generated. The gradient of the electric field is maximized at the edges of the IDE structures. Consequently, particles can be immobilized on the electrodes. To optimize the efficiency of the IDEs and DEP force for immobilizing different particle sizes, numerical simulations were done to study the effect of electric potential, frequency, fluid flow, and geometrical parameters of the IDE on particle tracing in a feasible manner. Different geometrical parameters of the IDE, such as finger width and 
spacing, were modeled. Finite element analysis was performed to evaluate the best parameters for dielectrophoretic immobilization. The first simulation model for the particle tracing was presented previously [23]. The details of the simulation with the extended versions are presented in this work.

\section{Sensing and System Overview}

This section describes the subsets of the advanced technological process required for enabling the dielectrophoretic immobilization of the bio-particles. The details of the IDE prototype used for manipulation and sensing as well as the readout detection circuit design along with the silicon microfluidic channel integration technology are presented below.

\subsection{Silicon Interdigitated Capacitor Sensor}

The IDE used in this work is similar to a sensor previously established for nearfield biosensors [14,15]. The fabrication of the IDE was performed based on the standard $250 \mathrm{~nm}$ high-performance SiGe BiCMOS technology of IHP. This sensor unit is embedded in the microfluidic channel and relies on the multi-fingered planar interdigitated capacitor arrays (see Figure 1), which are fabricated on the topmost metal level of the back-end-of-line (BEOL) stack of the CMOS/BiCMOS process. This IDE is used to model the dielectrophoretic structures.

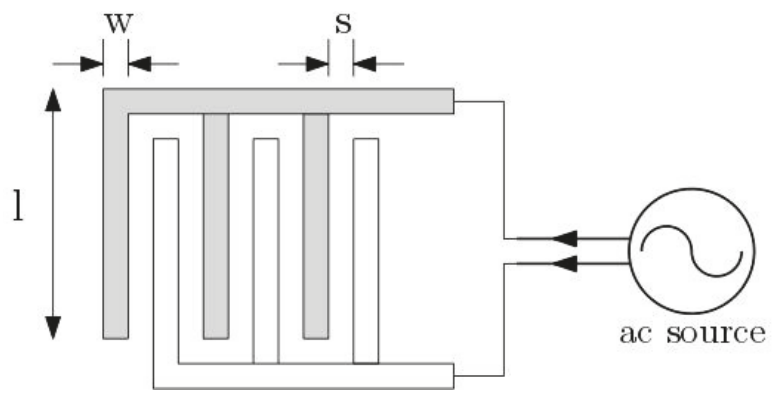

Figure 1. Multi-fingered planar interdigitated electrodes (IDEs).

As shown in Figure 2a, the magnitude of the fringing electric field is enlarged between two adjacent fingers of the IDE. As a result of the increased gradient of the E-Field at rectangular electrode corners, the distribution of the E-Field is inhomogeneous [24]. This effect leads to attraction and trapping of the particles to this region. As shown in Figure $2 b$, this E-Field magnitude exponentially decays with increasing distance to the IDE arrays. To visualize the simulated electric field, a cut line 2D data set was used to create lines through the modeled 2D geometry, as shown in cross-sectional schematic figures below. The electric field has been plotted along the red arrow-line, Figure 2a,b. The distribution of the electric field is calculated based on Equation (10).

The sensing principle of the IDE is based on the variation of the fringing electric field between the fingers of the IDE due to the permittivity change of the material (medium), as shown in Figure 3a.

The capacitance of the fringing field can be analyzed based on the quasi-static approximation of Maxwell's Equation [18]. The total capacitance of the two consecutive electrode fingers is equal to the sum of the contributing capacitors, as shown in Equation (1) [14]. This capacitance results from the penetration of the fringing field into the MUT as well as substrate and influence of the finger height on parallel plate capacitance, Figure $3 \mathrm{~b}$.

$$
C_{\text {total }}=C_{\text {MUT }}+C_{\text {Substrate }}+C_{\text {Oxide }} \text {. }
$$


The capacitance of the fringing filed concerning MUT and substrate is given as below [17].

$$
C_{\text {MUT }}+C_{\text {Substrate }}=\varepsilon_{0}\left(\frac{\varepsilon_{\text {substrate }}+\varepsilon_{M U T}}{2}\right)\left(\frac{K \sqrt{1+k^{2}}}{K(k)}\right),
$$

where $K(k)$ is the parameter arising due to the conformal mapping technique. The change in total capacitance is governed by the permittivity of the MUT, as shown in Equation (2).

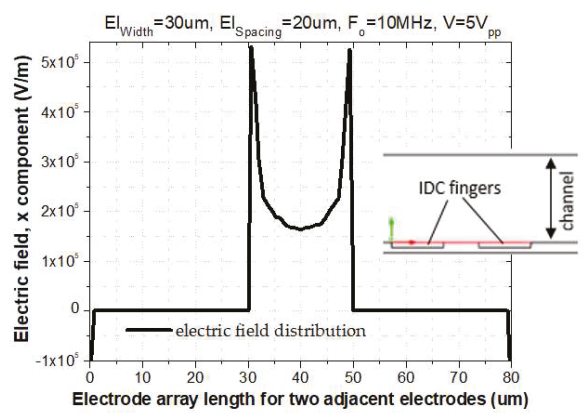

(a)

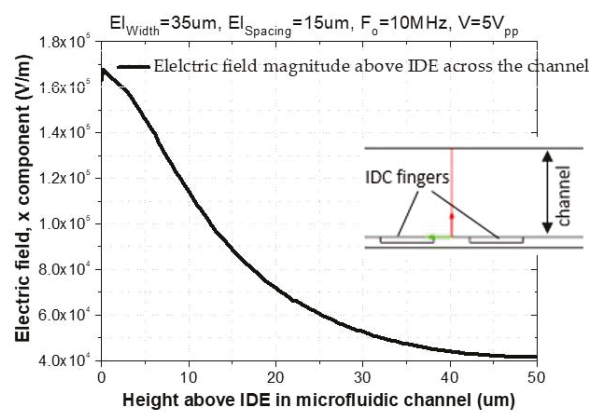

(b)

Figure 2. (a) E-Field distribution above two adjacent fingers of IDE; (b) Electric field magnitude as a function of distance above IDEs.

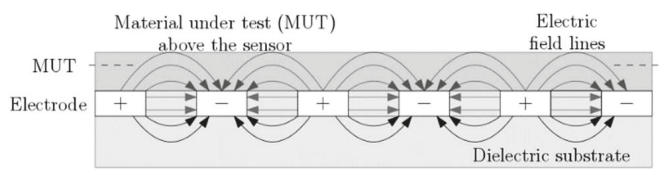

(a)

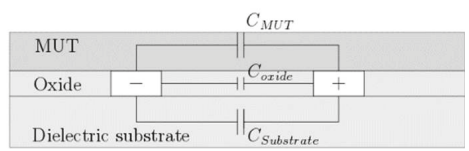

(b)

Figure 3. Interdigitated electrode: (a) Electric field distribution of the IDE; (b) Equivalent capacitive contributions from IDE and material-under-test (MUT).

The sensing operation of the IDE relies on the electrodynamics of the parallel plate capacitors. Applying an AC voltage to positive and negative terminals, a non-un electric field is created in the vicinity of the IDE. This electric field impacts the trajectory of the particles which are suspended in the medium above the IDE passing through the microfluidic channel. The capacitance of the IDE (sensor) changes, as a result of the permittivity change and its geometry [25]. Thus, the varying value of the IDE's capacitance alters the impedance of the IDE which can, in turn, be deduced through impedance spectroscopy. One such readout architecture which characterizes a sensor is a reflectometer. It measures the change in reflection amplitude and phase from a sensor (here IDE), which is a function of the change in sensor impedance. This readout scheme is elaborated further in the succeeding sub-section.

\subsection{Readout Circuit Architecture}

The prospective architecture of the silicon integrated readout circuit is briefly described in this section. Deployment of a high frequency $60 \mathrm{GHz}$ read-out circuit presented in [26] is projected as it allows for miniaturization of sophisticated read-out architectures. The readout circuit is chosen to operate at $60 \mathrm{GHz}$ for it provides a good trade-off between miniaturization and power consumption. A low-frequency implementation of the reflectometer will require a large die area. A homodyne reflectometric scheme to sensor read-out will be accomplished, as shown in Figure 4a, in that the local oscillator (LO) and RF input to the mixers are the same frequency. The major design blocks include a millimeter wave oscillator source, down active conversion mixers, and high-frequency wideband 
directive elements realized as passives. The two major passives are distributed branch line couplers and Wilkinson power dividers. These have been designed on the topmost metal layer of the technology stack to minimize the transmission losses. The two critical power splitters deployed and indicated as branch line couplers split the input source power for source and reflection characterization. The architecture in Figure 4a comprises two major channels viz. source characterization I/Q mixer channel (I1-Q1) and the reflection characterization I/Q mixer channel (I2-Q2). The source oscillator will excite the load, which is an interdigitated capacitor sensor. Based on the dielectrophoretic action, the immobilized particles will cause a change in impedance of the sensor, which will, in turn, produces a corresponding change in the reflection parameters captured by the reflection channel. Thus, a varying dielectric sample on this sensor will induce a corresponding change in the magnitude and phase of the reflected signal which can be deduced from the reflection channel dc output voltages. The mixers form the major constituent in this architecture and are described here briefly. An active double balanced Gilbert cell mixer is used, and its schematic diagram is shown in Figure 4b. The transistors Q1-Q2 constitutes the input transconductance stage of the mixer. The cascode stage constituting two pairs of transistors, i.e., Q3-Q4 and Q5-Q6, forms the commutating switch. Since the architecture is a homodyne reflectometer, the outputs are dc voltage and are read across the resistive load RL of the mixer. For a possible operating condition of a LO drive of $-2.7 \mathrm{dBm}$ and an incident RF power of $-19 \mathrm{dBm}$, the transfer characteristics for an RF phase sweep in the simulation is shown in Figure 4c.

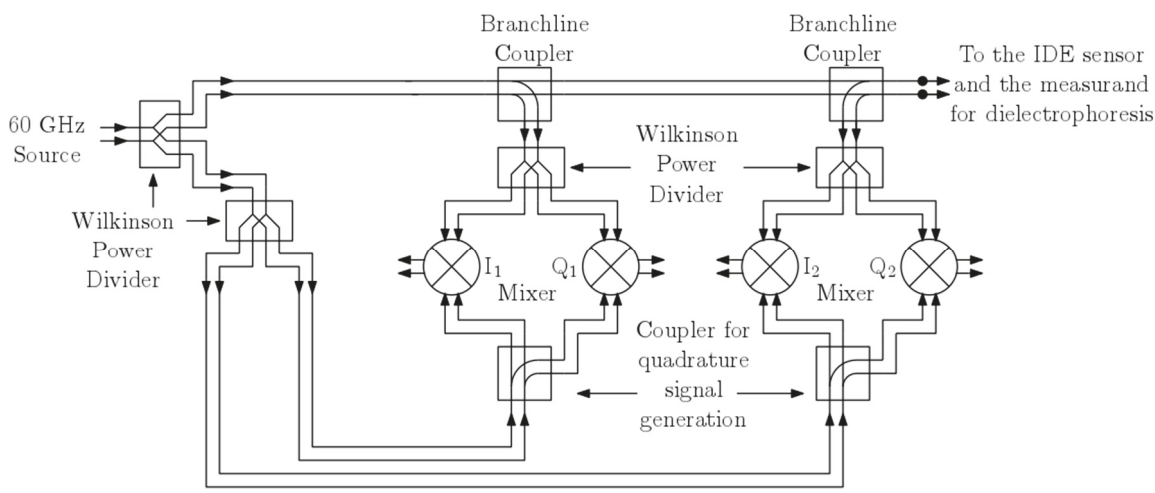

(a)

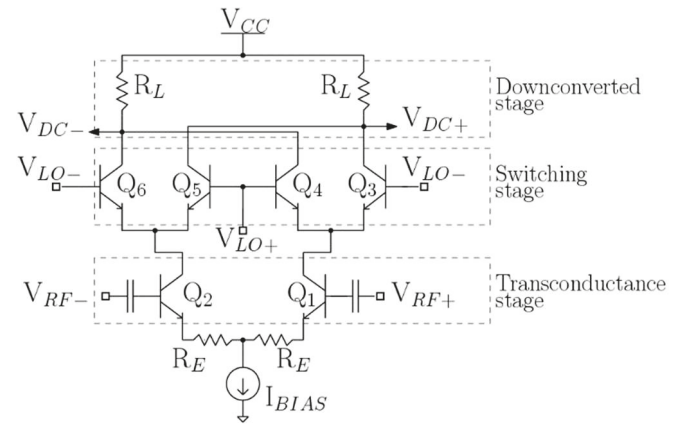

(b)

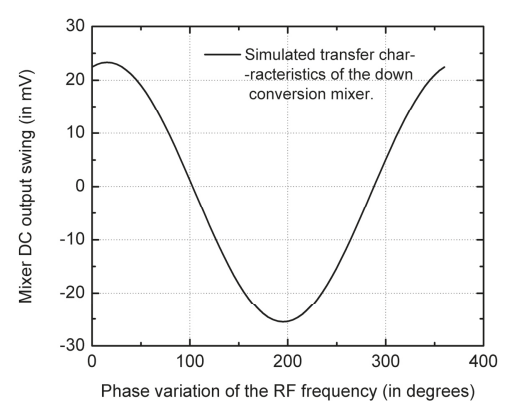

(c)

Figure 4. (a) High frequency $60 \mathrm{GHz}$ integrated homodyne reflectometer; (b) The Gilbert-cell mixer; (c) Sample transfer function characteristics of the mixer.

The integration of the sensor and the high-frequency readout will be accomplished through a dual excitation scheme, which is beyond the scope of this discussion. The measurement noise floor of 
this architecture is merely governed by the $1 / \mathrm{f}$-noise of the mixers as it is a homodyne reflectometer architecture. Since the heterojunction bipolar transistors (HBTs) have a lower 1/f-noise corner frequency than CMOS, the deployed mixer-based architecture will offer superior noise performance. Additionally, the load resistance of the mixer also contributes to the total output noise. The observed total output noise in the simulation is found to be $2.2 \mathrm{mV}_{\mathrm{rms}}$. This governs the minimum cell concentration, which can be reliably handled with the reflectometric readout circuit.

\subsection{Silicon Microfluidic Integration}

The CMOS IDE, as shown in Figure 5a, is combined with a microfluidic channel on a single chip (see Figure 5b). This CMOS integrated microfluidic technology platform improves the functionality and precision by combining the fluidic solution and electrical components. The major step in the integration of the microfluidic channel into the CMOS process is the lines similar to [27] and is briefly repeated. The technology approach integrates CMOS circuitry with microfluidics, based on the 3-wafer-stack technique in a CMOS compatible manner.

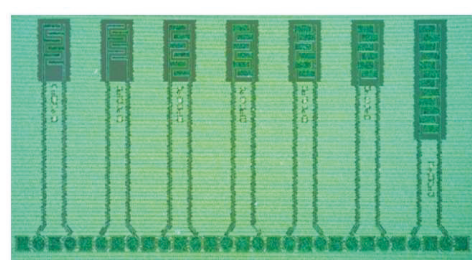

(a)

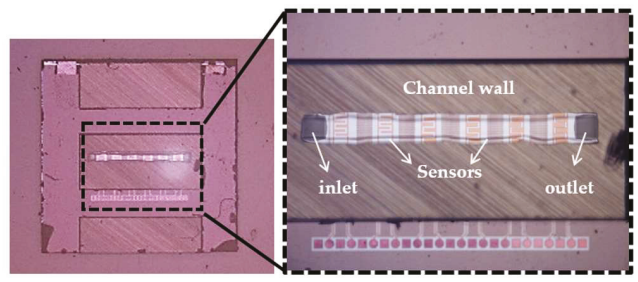

(b)

Figure 5. (a) Processed IDEs (sensors) structures b; (b) Complementary metal-oxide-semiconductor (CMOS) integrated microfluidic lab-on-a-chip device with size of $5 \times 5 \mathrm{~mm}^{2}$.

State-of-the-art of our integrated silicon microfluidic CMOS lab-on-a-chip is based on the compatibility of IHP's CMOS as well as hetero-integration technology of the microfluidic device. The compatibility of CMOS technology can be explained in two aspects. First, how to choose new materials to prevent contamination issues. Second, the CMOS line packaging tool, a so-called pilot package line. The fabrication of CMOS integrated microfluidics is performed without contamination problems of the CMOS process line. The hetero-integrated CMOS technology enables the fabrication of sensors, microfluidic channel, and circuitry on a single chip. This Si-based microfluidic channel is a reliable replacement for the polymer-based microfluidic channel that lacks from the integration of the sensor and circuitry on the same chip. The integration of CMOS circuitry, silicon channels, and glass wafers is done by $200 \mathrm{~mm}$ wafer bonding technologies. First, the fabricated the CMOS wafer and the channel wafer are bonded by plasma activated oxide-oxide fusion bonding, followed by adhesive bonding of a glass wafer.

Figure 6 illustrates the entire process flow for microfluidic devices. The wafer with the CMOS devices, including the BEOL sensors is processed on $200 \mathrm{~mm} \mathrm{Si} \mathrm{substrates,} \mathrm{Figure} 6 \mathrm{a}(1,2)$. In the next step, the inlet and outlet ports are opened from the back side of the wafer by localized backside etching, Figure 6a (3). Another bare silicon substrate is used to structure the channel by etching, Figure 6b. The Si-based microfluidic channel and the CMOS wafer are bonded together from their front sides using a low-temperature fusion bonding at $300{ }^{\circ} \mathrm{C}$, Figure $6 \mathrm{c}$ (1). After this step, the height of the channel is adjusted by grinding the backside of the microfluidic channel, Figure 6c (2). Finally, for encapsulating of the microfluidic channel, a glass wafer is adhesively bonded to the back side of the channel wafer at $200{ }^{\circ} \mathrm{C}$, Figure $6 c$ (3) [28]. The transparency of the top layer enables the simultaneous optical investigation and electrical sensing measurements. Figure $5 \mathrm{~b}$ illustrates the fabricated $5 \times 5 \mathrm{~mm}^{2}$ diced chip with the inlet and outlet and the sensors which are embedded in the channel. One of the 
noteworthy advantages of using this microfluidic integration technique is the separate interfaces for microfluidics and the electrical connections, enabling highly miniaturized LOC systems. State-of-the-art of this BiCMOS technology offers the opportunity of immobilizing, sensing, and detecting the particles on the same chip. The operational simplicity and low voltage requirement of the DEP technique, as well as the small volume of sample for testing, facilitate the portability of the LOC devices even out of the non-laboratory conditions.

(a)

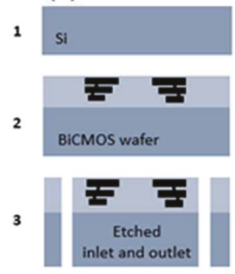

(b)

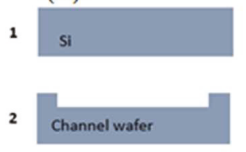

(c)
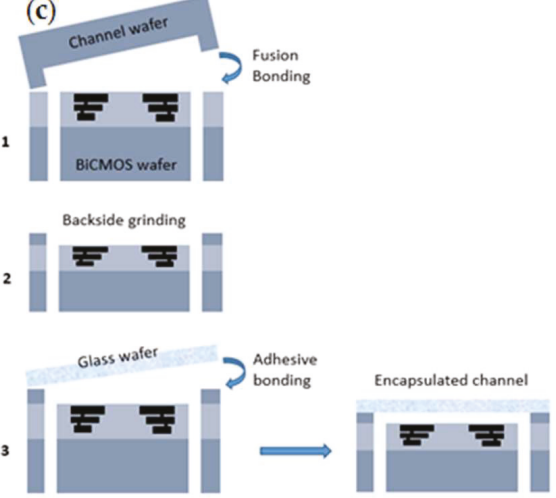

Figure 6. Packaging process of microfluidic lab-on-a-chip: (a) CMOS fabrication; (b) Channel formation; (c) Bonding process by a three-wafer-stack approach [28].

\section{DEP Analyses and Optimization}

Based on the COMSOL Multiphysics ${ }^{\circledR}$ tool, 2D and 3D models are developed. To simulate the dielectrophoretic immobilization of particles suspended in an aqueous solution, the fluid flow is considered through a microfluidic channel with a single inlet and a single outlet. Arrays of IDEs are utilized to create a non-uniform electric field that impacts the trajectory of particles due to dielectrophoretic forces. The DEP force was optimized with respect to different geometrical parameters of electrodes, size of the particle, and flow velocity. Figure 7a illustrates the device design in 3D geometry, while the simulation studies were all done in $2 \mathrm{D}$ to reduce the computational time, as illustrated in Figure $7 \mathrm{~b}$. In this simulation, different geometrical parameters of electrodes, such as width and spacing between adjacent fingers of IDE, was studied. The electric current (ec) module and particle tracing for fluid flow (fpt) in conjugation with drag and dielectrophoretic forces modules were used as the physics interfaces [29].

Different equations were used to simulate the flow path of the particles suspended in the fluidic medium and subsequently trapping them on the electrodes by DEP. The creeping flow (spf) module was used to model the fluid flow through the channel. Fluid velocity within the channel was determined based on the Navier-Stokes Equation shown in (3).

$$
0=\nabla \cdot\left[-p l+\mu(\nabla u+(\nabla u) T)-\frac{2}{3} \mu(\nabla \cdot u) l\right]+F \nabla \cdot(\rho u)=0 .
$$

Here, $p$ is the pressure, $u$ is the velocity vector, $\mu$ is the dynamic viscosity, and $F$ is the volume force vector that is acting on the fluid. When a particle is suspended in the fluid, it is affected by several forces. One of these forces, is called the drag force, and is caused by the fluid flow and has the same direction as the flow. The drag force is calculated based on Stokes drags law, which is also applicable for creeping flows $\left(\operatorname{Re}_{\mathrm{r}} \ll 1\right)$, as shown in Equations (4) and (5). 


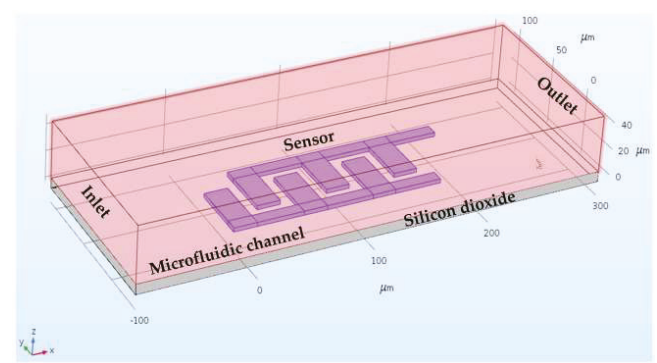

(a)

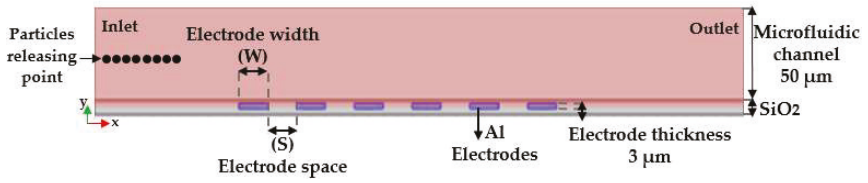

(b)

Figure 7. (a) 3D-geometry and; (b) 2D-geometry of the model device used to study the dielectrophoretic forces on particle tracing.

$$
\begin{gathered}
F_{\text {Drag }}=\left(\frac{1}{\mathrm{~T}_{p}}\right) \cdot m_{p}(u-v), \\
\tau_{p}=\rho_{p} \cdot \frac{d_{p}{ }^{2}}{18 \mu},
\end{gathered}
$$

where $\tau_{p}$ is the velocity response time of particles, $m_{p}$ is the particle mass, $v$ velocity of the particles, $u$ fluid velocity, $\mu$ fluid viscosity, $\rho_{p}$ particle density, and $d_{p}$ particle diameter. Furthermore, to attract and trap the particles, the dielectrophoretic force $\left(F_{D E P}\right)$ is required, which is given by Equation (6). The particles were subjected to a non-uniform AC electric field.

$$
\begin{gathered}
F_{D E P}=2 \pi r_{p}{ }^{3} e_{f} \operatorname{real}(C M) \nabla|E|, \\
C M=\frac{\left(e_{p}{ }^{*}-e_{f}^{*}\right)}{\left(e_{p}{ }^{*}+2 e_{f}^{*}\right)}, \\
\varepsilon_{f}^{*}=e_{r}-\left(\frac{i \sigma}{\omega}\right) .
\end{gathered}
$$

Here, $r_{p}$ is the particle radius, $\varepsilon_{f}$ is the relative permittivity of the medium, $\varepsilon_{f}^{*}$ and $\varepsilon_{p}^{*}$ are the complex permittivities of the fluid and particles, respectively. $E$ is the root-mean-square of electric field strength. Permittivity is a complex quantity which is a function of the electric field's angular frequency $(\omega)$ and conductivity $(\sigma)$. Based on the electric potential $(U)$ which is applied to the electrodes, the electric field E, is simulated by the ec module and is calculated based on Equation (9).

$$
E=\nabla v .
$$

The fpt module, together with the particle tracing, contains the equations governing the motion and trajectory of particles in the fluid under the influence of a DEP force and drag force. By ignoring the gravity force, buoyancy and Brownian motion forces, the relevant equation can be written, as shown in (10). 


$$
m_{p} \frac{d v}{d t}=F_{D E P}-F_{D r a g}=F_{T},
$$

where, $m_{p}$ is the mass of particles, $v$ is the velocity of the fluid, and $F_{T}$ is the total force acting on the particle.

An electrically insulated boundary condition is applied to the fluidic boundary, which is active on all exterior boundaries of the model. Negative and positive electric potentials were imposed on the electrodes at the bottom of the microfluidic channel. The electric potential of zero Volt was then applied to the electrodes as a further initial condition. The wall conditions were imposed without slip. No slip boundary condition $(u=0)$ was used to model solid walls and bounce for modeling the tracing of microscopic particles in the fluid. The position of the inlet and the outlet were perpendicular to the device structure. The boundary condition for the fluid flow at the inlet was set as velocity and at the outlet was set as pressure. At the inlet, the initial fluid flow velocity $\left(v_{0}\right)$ was set to $50 \mu \mathrm{m} / \mathrm{s}$. This value was then varied to study its impact on the immobilization of particles. To establish a fluid pressure gradient in the channel, an initial fluid velocity was applied at the inlet and the outlet was kept to zero fluid velocity with suppressing backflow of the medium. For tracing the particles, disappearing or freezing wall conditions were selected at the outlet. The disappearing mode was used when the particles leave the channel in case of deviation from the electrodes. The freezing wall condition was used to study the velocity and the position of particles, where particles could stay frozen at the point where they leave the channel outlet boundary.

The simulation analysis was done in three main steps. The electric current module, which simulates the electrical potential field, was applied to the electrode to create a non-uniform electric field. A stationary analysis was performed to simulate the flow velocity through the channel, as illustrated. Particle tracing module was used to model dielectrophoretic force through the channel. Figure 8 describes an example of the simulation analysis steps for $3.5 \mu \mathrm{m}$ diameter sized particles. As shown in Figure 8a, negative and positive voltage peak values of $2.5 \mathrm{~V}$ and $-2.5 \mathrm{~V}$ were applied to the electrodes. Therefore, the total applied electric potential was $5 \mathrm{~V}$ peak to peak voltage $\left(U=5 \mathrm{~V}=\mathrm{V}_{\mathrm{p}-\mathrm{p}}\right)$. Figure $8 \mathrm{~b}$ shows the electric field contour plot. Figure $8 \mathrm{c}$ illustrates the constant fluid flow velocity in the middle part of the channel. The pressure was almost constant in the channel. For particle tracing a time-dependent solution was performed using the values obtained from the frequency domain analysis, as shown in Figure 8d.

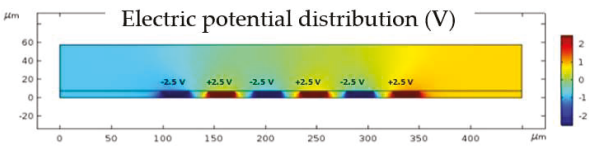

(a)

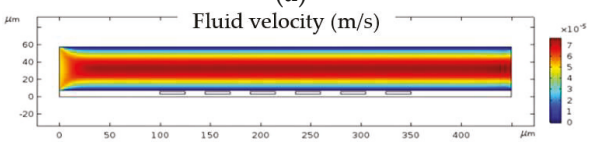

(c)

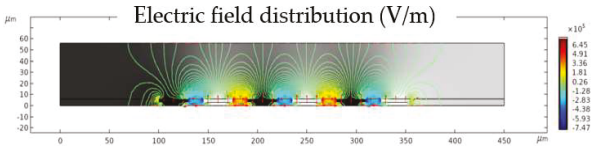

(b)

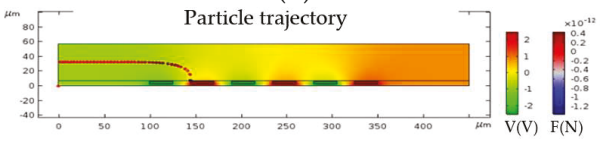

(d)

Figure 8. Simulation analysis steps for $3.5 \mu \mathrm{m}$ diameter size particles: (a) Electric potential distribution in the microfluidic channel at $U=5 \mathrm{~V}$ at a fixed frequency of $10 \mathrm{MHz}$; (b) Electric field distribution contour plot in the microfluidic channel; (c) Velocity field across the microfluidic channel; (d) Particle trajectories with respect to the applied electric potential and the force acting on the particles leading to the immobilization of the particles at the surface of electrodes.

Since the objective of this study is to immobilize the particles on the electrodes, simulations were performed systematically to optimize the electrode geometry and to find suitable electric field frequency ranges and fluid velocities to attract different size particles. 
The particle sizes used for these simulation studies were in micron and submicron-diameter ranges $(10,3.5,0.5)$. These particles were used as models and assumed as homogeneous spheres and non-biological. To keep the first model simple and reduce the complex parameters, it was assumed that these particles were diluted in water. Therefore, the osmotic pressure effect can be neglected for these simulations. Table 2, represents the properties of the particles for the simulations.

Table 2. Particle properties.

\begin{tabular}{ccccc}
\hline MUT $^{\mathbf{1}}$ & Particle Diameter $(\boldsymbol{\mu m})$ & Density $\left.\mathbf{( k g} / \mathbf{m}^{\mathbf{3}}\right)$ & Permittivity & Conductivity $(\mathbf{S} / \mathbf{m})$ \\
\hline Particle 1 (platelet) & 3.5 & 1000 & 50 & 25 \\
Particle 2 & 0.5 & 1000 & 50 & 25 \\
Particle 3 & 10 & 1050 & 5 & 0.69 \\
(fluorescent) & & & & \\
\hline
\end{tabular}

${ }^{1}$ Material Under Test.

\subsection{Impact of Frequency on DEP}

The choice of the operating frequency, which leads to immobilization of the particles, was based on the Clausius Mossotti (CM) factor. CM is a frequency dependent parameter which results in negative and positive DEP. Changing the frequency stimulate the crossover frequency between positive and negative DEP. The value of the real part is varied between +1 and -0.5 . When the real part of the $C M$ factor is positive, positive DEP forces act on the particle, which results in the attraction of them to the electric field intensity maxima, i.e., electrode edges. In the opposite case (real $[\mathrm{CM}]<0$ ), particles are repelled from high electric field intensity regions and consequently deviated from the electrode. To find a suitable frequency range, the CM factors of the particles were calculated by using MATLAB ${ }^{\circledR}$. Figure 9 illustrates the $\mathrm{CM}$ factor for $10 \mu \mathrm{m}$ diameter size fluorescent particles. Particle properties are shown in Table 1. At positive values of the Clausius Mossotti factor (real $[\mathrm{CM}]>1$ ), particles are trapped on the electrodes. The positive DEP is caused by the positive values of $\mathrm{CM}$. At negative real values $[\mathrm{CM}]<0$, negative force is acting on the particles. This leads to the deviation of particles from the electrodes. Therefore, for reliable DEP immobilization, frequencies below $10 \mathrm{MHz}$ must be chosen.

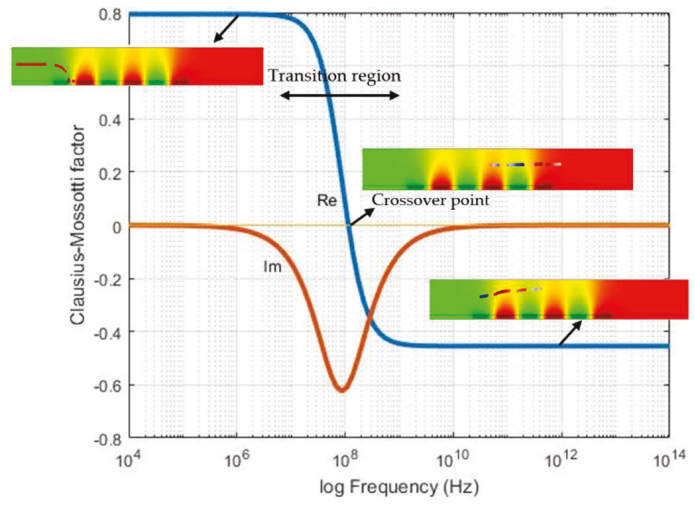

Figure 9. Clausius Mosotti (CM) factor for $10 \mu \mathrm{m}$ diameter-sized particle as a function of frequency.

\subsection{Impact of Voltage Variation and Particle Size}

The simulated results of the improved particle tracing for 3.5 and $0.5 \mu$ m diameter-size particles are shown in Figure 10. The immobilization of particles was positively correlated with the voltage. For the same IDE structure, the immobilization of $3.5 \mu \mathrm{m}$ size particles started already at $1 \mathrm{~V}$, Figure $10 \mathrm{a}$, while the immobilization of $0.5 \mu \mathrm{m}$ particles occurred at $8 \mathrm{~V}$, shown in Figure 10b. 
The dielectrophoretic force which is subjected to the particles by the electric field is positively correlated with the electric potential. In other words, for the trapping of smaller particles on electrodes, higher voltages are required to compensate the mechanical force and overcome the drag force from fluid flow.

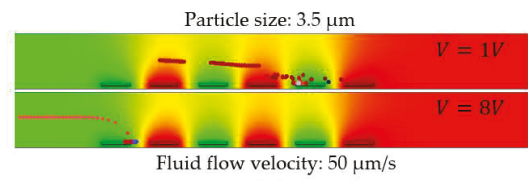

(a)

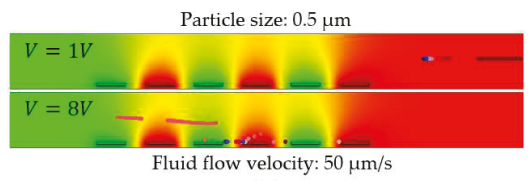

(b)

Figure 10. Impact of voltage variation on the DEP force for particles at constant fluid flow velocity $(50 \mu \mathrm{m} / \mathrm{s})$, geometrical parameters of IDEs (electrode width of, $30 \mu \mathrm{m}$, and spacing of, $20 \mu \mathrm{m}$ ) and DEP operating frequency (1 MHz). (a) Diameter size of $3.5 \mu \mathrm{m}$; (b) $0.5 \mu \mathrm{m}$.

\subsection{Impact of Fluid Flow Velocity}

The maximum operating voltage of IHP CMOS technology is constrained to 5 volts. To immobilize the submicron particle, the voltage was kept at CMOS compatible ranges, and the fluid flow velocity was varied. The fluid velocity is inversely impacted by the immobilization of small particles. Figure 11 shows a representative simulation result for reducing the fluid velocity at a constant voltage of $5 \mathrm{~V}$. The fluid flow velocity of $50 \mu \mathrm{m} / \mathrm{s}$ was set as the reference. It can be seen that reducing the fluid velocity to $30 \mu \mathrm{m} / \mathrm{s}$ improved the attraction of particles.

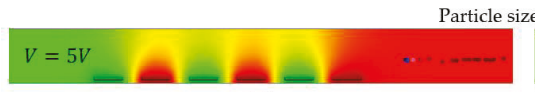

(a)

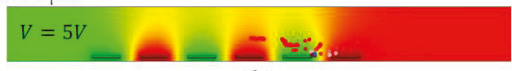

(b)

Figure 11. Influence of fluid flow velocity on tracing of the $0.5 \mu \mathrm{m}$ particles through the microfluidic channel at a constant voltage of $5 \mathrm{~V}$ and operating frequency of $1 \mathrm{MHz}$. At flow rates of: (a) $50 \mu \mathrm{m} / \mathrm{s}$; and (b) $30 \mu \mathrm{m} / \mathrm{s}$.

\subsection{Impact of IDE's Geometry on DEP Immobilization Probability}

In the following simulation step, the voltage, flow velocity, and the frequency were kept constant. The impact of the geometrical dimensions of the IDE on the trajectory of particles is studied. The immobilization probability values of each IDE's geometrical design can be calculated by $\mathrm{COMSOL}^{\circledR}$ applications. Based on these analyses, the immobilization probability is defined as the ratio of the number of immobilized particles to the total number of particles suspended in the medium. The influence of the fringing field effect with different ratios of the electrode width and spacing $\left(\frac{S}{W}\right)$ versus immobilization probability is shown in Figure 12.

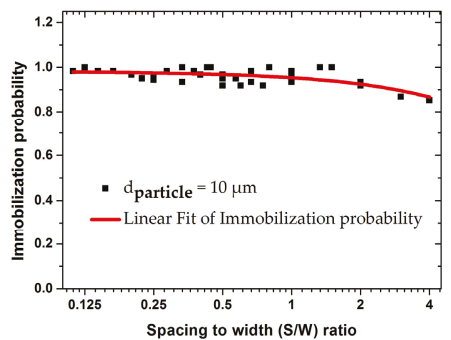

(a)

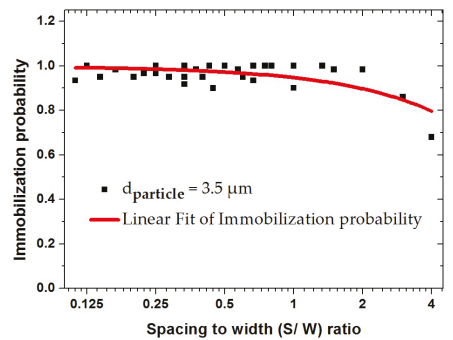

(b)

Figure 12. Finite element modeling (FEM) results for the immobilization probability with different ratios of the IDE's spacing and width $\left(\frac{S}{W}\right)$ for 10 and $3.5 \mu \mathrm{m}$-sized particles at $1 \mathrm{MHz}$ and fluid velocity of $50 \mu \mathrm{m} / \mathrm{s}$ : (a) $10 \mu \mathrm{m}$; (b) $3.5 \mu \mathrm{m}$ particle. 
The width varied between 5 and $45 \mu \mathrm{m}$, and the spacing varied between 5 and $20 \mu \mathrm{m}$. These graphs illustrate the immobilization probability (IP) of 10 and $3.5 \mu \mathrm{m}$ size particles. For both particle sizes, IP was larger than 0.9 for ratios below 2 . At higher ratios, IP values decreased for both particle sizes. This reduction becomes significant for smaller particles at a ratio of 4 . The IDE design was optimized when the ratio of spacing to width is smaller than 2. In the following section, this trend is explicitly discussed and illustrated in Figures 13 and 14.

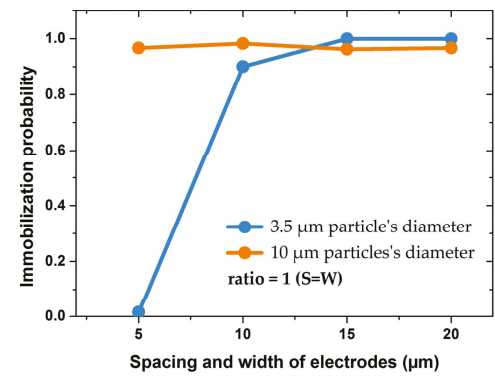

Figure 13. Impact of IDE's width in symmetric structures $(S=W)$ on immobilization probability of 10 and $3.5 \mu \mathrm{m}$ particles under the same voltage and fluid velocity.

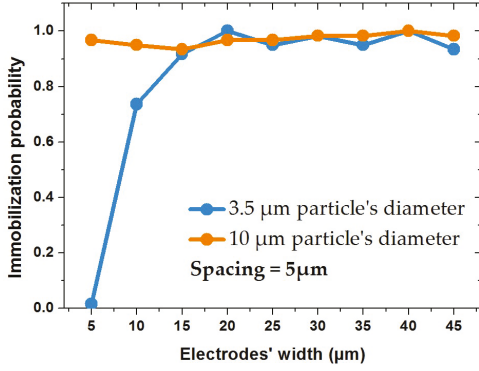

(a)

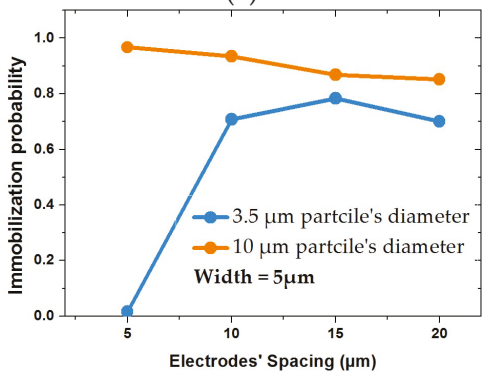

(c)

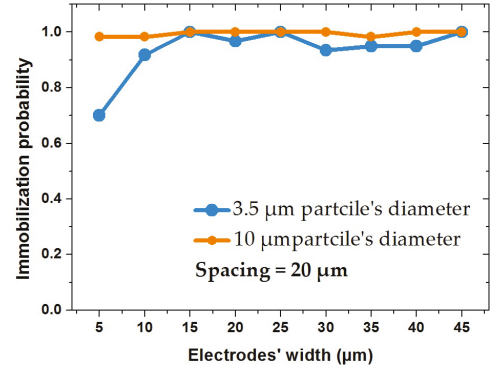

(b)

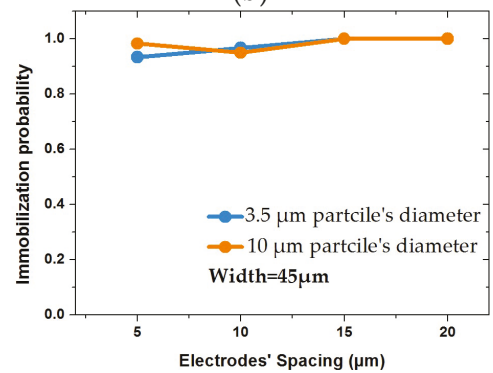

(d)

Figure 14. Impact of IDE's geometrical parameters on immobilization probability (IP) of particles with diameters of 10 and $3.5 \mu \mathrm{m}$, keeping voltages and fluid velocities constant: (a,b) illustrate IP variations with respect to different widths at fixed spacings of 5 and $20 \mu \mathrm{m}$, respectively; (c,d) show the impact of spacing size on IP at fixed widths of 5 and $45 \mu \mathrm{m}$, respectively.

In the following, the IDEs are assigned into two groups of uniform and non-uniform geometries. Uniform IDEs refers to geometries with equal sizes of width and spacing. Geometries with an unequal size of width and spacing are referred to as non-uniform IDEs. Figure 13 shows the IP values for uniform IDEs. 
Using the uniform electrode geometries, the immobilization probability for larger particles (10 $\mu \mathrm{m})$ was almost $100 \%$ in all cases. For smaller particles $(3.5 \mu \mathrm{m})$ IP increased when the electrodes width and spacing were larger than $10 \mu \mathrm{m}$. For IDEs with spacing and width of $5 \mu \mathrm{m}$, IP was zero for small particle, whereas for bigger particle it was almost 1 . Therefore, it can be concluded that the uniform IDEs design is optimized when spacing and width are larger than $10 \mu \mathrm{m}$.

Figure 14 illustrates the impact of non-uniform IDE structures on immobilization probability of particles with sizes of $10 \mu \mathrm{m}$ and $3.5 \mu \mathrm{m}$. Figure $14 \mathrm{a}, \mathrm{b}$ represent the IP at a constant spacing of 5 and $20 \mu \mathrm{m}$. Figure $14 \mathrm{c}$,d illustrate the IP at constant widths ( 5 and $45 \mu \mathrm{m}$ ) with respect to spacing. The variation of spacing and width has less impact on IP of $10 \mu \mathrm{m}$ size particles. However, for small particle, $3.5 \mu \mathrm{m}$, the results were different. Keeping the spacing constant at $5 \mu \mathrm{m}$, IP decreased drastically, as shown in Figure 14a. However, this effect inversely impacts the IP with respect to spacings, which were comparatively much larger than the width (ratios over 2), as shown in Figure 14c. It can be concluded that the impact of the geometrical parameters is dominant if small particles have to be immobilized. Increasing spacing positively impacts the trapping of small particles for non-uniform IDEs ratios $\left(\frac{S}{W}\right)$ below 2 . In other words, the fringing field effect positively affects the probability to immobilize smaller particles with increasing width.

Based on the results, the optimum geometrical parameters were selected and applied for the simulation of the submicron particle $(0.5 \mu \mathrm{m})$. These structures' parameter with their respective spacing to width ratios are shown in Table 3. For these simulations, the applied voltage was kept constant at CMOS compatible range $(5 \mathrm{~V})$, and the fluid flow velocity reduced to $30 \mu \mathrm{m} / \mathrm{s}$.

Table 3. Selected IDE geometries which are compatible with CMOS operating range (5 V).

\begin{tabular}{ccccccc}
\hline IDE Structure & Structure $\mathbf{1}$ & Structure 2 & Structure 3 & Structure 4 & Structure 5 & Structure 6 \\
\hline Electrode width $(\mathrm{W})$ & $45(\mu \mathrm{m})$ & $40(\mu \mathrm{m})$ & $35(\mu \mathrm{m})$ & $30(\mu \mathrm{m})$ & $20(\mu \mathrm{m})$ & $15(\mu \mathrm{m})$ \\
Electrode spacing $(\mathrm{S})$ & $5(\mu \mathrm{m})$ & $10(\mu \mathrm{m})$ & $15(\mu \mathrm{m})$ & $20(\mu \mathrm{m})$ & $20(\mu \mathrm{m})$ & $20(\mu \mathrm{m})$ \\
Ratio = S/W & 0.1 & 0.25 & 0.4 & 0.6 & 1 & 1.3 \\
\hline
\end{tabular}

The tracing behaviors of $0.5 \mu \mathrm{m}$ particles for selected geometries are shown in Figure 15 . The same tracing trends as micron-sized particles were observed for submicron-sized particles at reduced flow rates for structures with ratios below 1 . The decreased spacing to width ratios improved the tracing and immobilization of submicron size particles. Using higher ratios, particles were repelled from the electrodes and left the microfluidic channel.

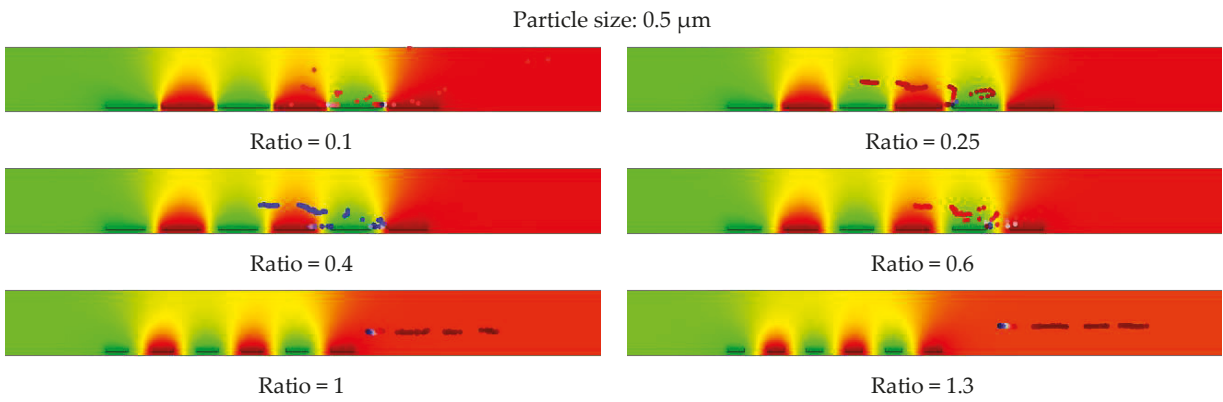

Figure 15. Impact of the optimized parameters (geometrical parameters and fluid flow velocity) on immobilization of submicron particles $(0.5) \mu \mathrm{m}$ at constant voltages of $5 \mathrm{~V}$ and fluid flow velocity of $30 \mu \mathrm{m} / \mathrm{s}$.

Therefore, it was observed that designing a better performance IDE is highly influenced by the optimum ratio of the electrode's spacing to width. Furthermore, the flow rate of the medium inside the microfluidic channel impacts the particles' movement behavior inside the electric field. 
These are important factors to consider for the immobilization of submicron and micron size particles. The optimized IDE parameters for immobilization of submicron particles are related to IDE geometries with ratios below one. However, for immobilization of micron size particles, the IDE is optimized for ratios below two.

\subsection{Impact of Temperature on DEP Process}

An insightful study of temperature effects on IDE parameters in the vicinity of room temperature is investigated though electromagnetic momentum simulation studies. Water was the liquid carrier of the particles, and thus, the impact of the prospective chip self-heating on the dielectric permittivity change is illustrated. Figure 16 shows the capacitance of the IDE due to change in water dielectric from room temperature up to $50{ }^{\circ} \mathrm{C}$. The frequency of observation remained $1 \mathrm{MHz}$, and the water dielectric layer thickness considered here was $50 \mu \mathrm{m}$ corresponding to the height of the microfluidic channel. The change in capacitance due to a temperature of up to $50{ }^{\circ} \mathrm{C}$ was found to be only up to $3.07 \%$. The temperature effects due to self-heating of the chip may be a pronounced issue for very long duration measurements only. However, the use of active circuit components is judicious to be used sparingly in the read-out circuit for particle detection. For instance, the source oscillator was an external signal source so that one of the power-consuming blocks is eliminated from the chip.

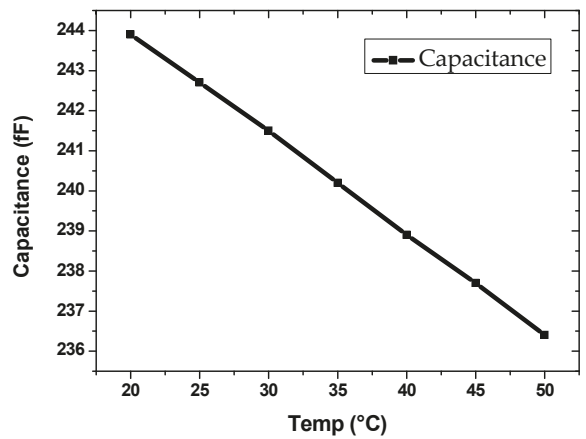

Figure 16. IDE's capacitance vs. water dielectric permittivity [30] at $1 \mathrm{MHz}$ excitation frequency, observed from room temperature up to $50{ }^{\circ} \mathrm{C}$.

The total power consumption was merely $64.3 \mathrm{~mW}$ and is contributed by the mixers. Additionally, the chip can be operated in a short duty cycle when the measurement of the change in capacitance is to be conducted. This ensures reliable operation which is minimally impacted by the chip-self heating and more so when the low power consumption readout circuit is placed away on the chip, relative to the sensors.

\section{Conclusions}

The viability of the CMOS embedded microfluidic device for dielectrophoretic immobilization of particles was investigated by finite element modeling (FEM) simulations. This study proposed a sensing platform to enable the immobilization of particles with micron and submicron sizes constrained by technological advances, such as silicon-based microfluidics and millimeter wave readout circuit. IDE's geometrical parameters were characterized and optimized based on essential external parameters, such as voltage, frequency, and velocity of the fluid through the microfluidic channel. By increasing the applied voltage trapping of smaller particles on electrodes is enhanced. The change of frequency does not influence the DEP force for improving the immobilization of particles but rather influences the crossover frequency between negative and positive DEP. Reducing the fluid flow velocity forces the immobilization of submicron particles. By keeping the voltage constant at CMOS compatible range, 
the impact of IDE's geometrical parameters on the tracing and immobilization of the particles was investigated. The ratio between the electrodes' spacing and the width impacts the IDE's performance. According to simulations, the width of the IDE's finger has to be increased, whereas the spacing has to be reduced. The simulation and design of planar IDEs were evaluated to maximize the immobilization probability of submicron sized particles. The IDE design for the immobilization of micron and submicron size particles with optimum geometrical parameters of IDE and the fluid flow rate was demonstrated. Furthermore, a study on the impact of temperature (also due to chip self-heating) in IDE parameters was investigated though electromagnetic momentum simulations. It was observed that the change in impedance of IDE is only up to $3.07 \%$ from room temperature up to $50{ }^{\circ} \mathrm{C}$ which is less likely to affect the short term measurements. Lastly, the presented optimized IDEs can be combined with various readout architectures. The $60 \mathrm{GHz}$ high-frequency reflectometer based architecture was chosen to enable higher miniaturization of a sophisticated sensing platform, and its homodyne architecture will enable a dc-in and dc-out based impedance spectroscopy.

Author Contributions: Methodology, H.M.E. and S.G.; Validation, H.M.E., R.K.Y. and S.G.; Software, H.M.E. and R.K.Y.; Formal analysis, H.M.E., R.K.Y. and C.W.; Data curation, H.M.E., R.K.Y. and S.G.; Writing-original draft preparation, H.M.E.; Writing-review \& editing, H.M.E., R.K.Y. and C.W.; Supervision, C.W.; Funding acquisition, C.W.

Funding: This research was funded by the Brandenburg Ministry of Science, Research, and Cultural Affairs for funding the project within the StaF program in Germany.

Acknowledgments: The authors would like to thank the technology department of IHP for the fabrication of the sensor chip.

Conflicts of Interest: The authors declare no conflict of interest.

\section{References}

1. Pohl, H.A.; Crane, J.S. Dielectrophoresis of cells. Biophys. J. 1971, 11, 711-727. [CrossRef]

2. Çetin, B.; Li, D. Dielectrophoresis in microfluidics technology. Electrophoresis 2011, 32, 2410-2427. [CrossRef]

3. Srivastava, S.K.; Gencoglu, A.; Minerick, A.R. DC insulator dielectrophoretic applications in microdevice technology: A review. Anal. Bioanal. Chem. 2011, 399, 301-321. [CrossRef]

4. Cheng, I.F.; Chang, H.C.; Hou, D.; Chang, H.C. An integrated dielectrophoretic chip for continuous bioparticle filtering, focusing, sorting, trapping, and detecting. Biomicrofluidics 2007, 1, 021503. [CrossRef]

5. Li, H.; Zheng, Y.; Akin, D.; Bashir, R. Characterization and modeling of a microfluidic dielectrophoresis filter for biological species. J. Microelectromech. Syst. 2005, 15, 103-112. [CrossRef]

6. Markx, G.H.; Talary, M.S.; Pethig, R. Separation of viable and non-viable yeast using dielectrophoresis. J. Biotechnol. 1994, 32, 29-37. [CrossRef]

7. Otto, S.; Kaletta, U.; Bier, F.F.; Wenger, C.; Hölzel, R. Dielectrophoretic immobilisation of antibodies on microelectrode arrays. Lab Chip 2014, 14, 998-1004. [CrossRef]

8. Khan, S.M.; Gumus, A.; Nassar, J.M.; Hussain, M.M. CMOS Enabled Microfluidic Systems for Healthcare Based Applications. Adv. Mater. 2018, 30, 1-26. [CrossRef] [PubMed]

9. Tran, D.P.; Pham, T.T.T.; Wolfrum, B.; Offenhäusser, A.; Thierry, B. CMOS-compatible silicon nanowire field-effect transistor biosensor: Technology development toward commercialization. Materials 2018, 11, 785. [CrossRef] [PubMed]

10. Huang, Y.; Mason, A.J. Lab-on-CMOS integration of microfluidics and electrochemical sensors. Lab Chip 2013, 13, 3929-3934. [CrossRef] [PubMed]

11. Welch, D.; Christen, J.B. Seamless integration of CMOS and microfluidics using flip chip bonding. J. Micromech. Microeng. 2013, 23, 035009. [CrossRef]

12. Laux, E.; Knigge, X.; Bier, F.F.; Wenger, C.; Hölzel, R. Aligned immobilization of proteins using AC electric fields. Small 2016, 12, 1514-1520. [CrossRef] [PubMed]

13. Guha, S.; Farabi, F.I.; Schmalz, K.; Wenger, C.; Meliani, C. CMOS lab on a chip device for dielectric characterization of cell suspensions based on a $6 \mathrm{GHz}$ oscillator. In Proceedings of the European Microwave Conference (EuMC), Nuremberg, Germany, 6-10 October 2013; pp. 471-474. 
14. Guha, S.; Lisker, M.; Trusch, A.; Wolf, A.; Meliani, C.; Wenger, C. 12 GHz CMOS MEMS lab-on-chip system for detection of concentration of suspended particles in bio-suspensions. In Proceedings of the Biodevice 2015, Lisbon, Portugal, 12-15 January 2015; pp. 49-57.

15. Guha, S.; Warsinke, A.; Tientcheu, C.M.; Schmalz, K.; Meliani, C.; Wenger, C. Label free sensing of creatinine using a $6 \mathrm{GHz}$ CMOS near-field dielectric immunosensor. Analyst 2015, 140, 3019-3027. [CrossRef] [PubMed]

16. Manaresi, N.; Romani, A.; Medoro, G.; Altomare, L.; Leonardi, A.; Tartagni, M.; Guerrieri, R. A CMOS Chip for Individual Cell Manipulation and Detection. IEEE J. Solid-State Circuits 2003, 38, 2297-2305. [CrossRef]

17. Miled, M.A.; Sawan, M. Dielectrophoresis-Based Integrated Lab-on-Chip for Nano and Micro-Particles Manipulation and Capacitive Detection. IEEE Trans. Biomed. Circuits Syst. 2012, 6, 120-132. [CrossRef] [PubMed]

18. Park, K.; Suk, H.J.; Akin, D.; Bashir, R. Dielectrophoresis-based cell manipulation using electrodes on a reusable printed circuit board. Lab Chip 2009, 9, 2224-2229. [CrossRef] [PubMed]

19. Guha, S.; Schumann, U.; Jamal, F.I.; Wagner, D.; Meliani, C.; Schmidt, B.; Wenger, C.; Wessel, J.; Detert, M. Integrated high-frequency sensors in catheters for minimally invasive plaque characterization. In Proceedings of the 20th European Microelectronics and Packaging Conference and Exhibition: Enabling Technologies for a Better Life and Future (EMPC), Friedrichshafen, Germany, 14-16 September 2015; pp. 1-6.

20. Guha, S.; Schmalz, K.; Meliani, C.; Wenger, C.; Krautschneider, W. CMOS MEMS based Microfluidic System for Cytometry at $5 \mathrm{GHz}$. In Proceedings of the MFHS, Microfluidic handling System, Enschede, The Netherlands, 10-12 October 2012.

21. Guha, S.; Wenger, C. Radio Frequency CMOS Chem-Bio Viscosity Sensors based on Dielectric Spectroscopy. In Proceedings of the 10th International Joint Conference on Biomedical Engineering Systems and Technologies, SCITEPRESS—Science and Technology Publications, Porto, Portugal, 21-23 February 2017; pp. $142-148$.

22. Guha, S.; Schmalz, K.; Wenger, C.; Herzel, F. Self-calibrating highly sensitive dynamic capacitance sensor: Towards rapid sensing and counting of particles in laminar flow systems. Analyst 2015, 140, 3262-3272. [CrossRef] [PubMed]

23. Matbaechi Ettehad, H.; Guha, S.; Wenger, C. Simulation of CMOS compatible sensor structures for dielectrophoretic biomolecule immobilization. In Proceedings of the COMSOL-Bioscience and Bioengineering COMSOL, Rotterdam, The Netherlands, 19 October 2017; p. 6.

24. Kurgan, E.; Gas, P. An influence of electrode geometry on particle forces in AC dielectrophoresis. Prz. Elektrotechniczny 2010, 86, 103-105.

25. Guha, S.; Farabi, F.I.; Schmalz, K.; Wenger, C.; Meliani, C. An 8 GHz CMOS near field bio-sensor array for imaging spatial permittivity distribution of biomaterials. In Proceedings of the IEEE MTT-S International Microwave Symposium (IMS2014), Tampa, FL, USA, 1-6 June 2014.

26. Yadav, R.K.; Eissa, M.H.; Wessel, J.; Kissinger, D. A 60 GHz Mixer-Based Reflectometer in 130 nm SiGe BiCMOS Technology toward Dielectric Spectroscopy in Medical Applications. In Proceedings of the 2018 IEEE International Microwave Biomedical Conference (IMBioC), Philadelphia, PA, USA, 14-15 June 2018; pp. 88-90.

27. Inac, M.; Wietstruck, M.; Göritz, A.; Cetindogan, B.; Baristiran-kaynak, C.; Voß, T.; Mai, A.; Palego, C.; Pothier, A.; Kaynak, M. BiCMOS Integrated Microfluidic Packaging by Wafer Bonding for Lab-on-Chip Applications. In Proceedings of the 2017 IEEE 67th Electronic Components and Technology Conference (ECTC), Orlando, FL, USA, 30 May-2 June 2017; pp. 786-791.

28. Kaynak, M.; Wietstruck, M.; Baristiran-kaynak, C.; Marschmeyer, S.; Kulse, P.; Schulz, K.; Silz, H.; Kruger, A.; Barth, R.; Schmalz, K.; et al. BiCMOS integrated microfluidic platform for Bio-MEMS applications. In Proceedings of the IEEE MTT-S International Microwave Symposium (IMS2014), Tampa, FL, USA, 1-6 June 2014.

29. COMSOL Multiphysics ${ }^{\circledR}$ Reference Manual, v. 5.4. Available online: http://www.comsol.com (accessed on 4 June 2019).

30. Malmberg, C.G.; Maryott, A.A. Dielectric Constant of Water from $0{ }^{\circ} \mathrm{C}$ to $100{ }^{\circ} \mathrm{C}$. J. Res. Natl. Bur. Stand. 1956, 56, 1-8. [CrossRef]

(C) 2019 by the authors. Licensee MDPI, Basel, Switzerland. This article is an open access article distributed under the terms and conditions of the Creative Commons Attribution (CC BY) license (http://creativecommons.org/licenses/by/4.0/). 

Article

\title{
Acoustofluidic Micromixing Enabled Hybrid Integrated Colorimetric Sensing, for Rapid Point-of-Care Measurement of Salivary Potassium
}

\author{
Vikram Surendran ${ }^{1}$, Thomas Chiulli ${ }^{1}$, Swetha Manoharan ${ }^{1}$, Stephen Knisley ${ }^{1}$, \\ Muthukumaran Packirisamy ${ }^{2}$ and Arvind Chandrasekaran ${ }^{1, *}$ \\ 1 Department of Chemical, Biological and Bio Engineering, North Carolina A \& T State University, \\ Greensboro, NC 27411, USA; vsurendran@aggies.ncat.edu (V.S.); tjchiulli@aggies.ncat.edu (T.C.); \\ smanoharan@aggies.ncat.edu (S.M.); sbknisle@ncat.edu (S.K.) \\ 2 Department of Mechanical, Industrial and Aerospace Engineering, Concordia University, Montreal, \\ QC H2L5C9, Canada; pmuthu@alcor.concordia.ca \\ * Correspondence: achandra@ncat.edu; Tel.: +1-(336)-285-3721
}

Received: 22 April 2019; Accepted: 24 May 2019; Published: 28 May 2019

\begin{abstract}
The integration of microfluidics with advanced biosensor technologies offers tremendous advantages such as smaller sample volume requirement and precise handling of samples and reagents, for developing affordable point-of-care testing methodologies that could be used in hospitals for monitoring patients. However, the success and popularity of point-of-care diagnosis lies with the generation of instantaneous and reliable results through in situ tests conducted in a painless, non-invasive manner. This work presents the development of a simple, hybrid integrated optical microfluidic biosensor for rapid detection of analytes in test samples. The proposed biosensor works on the principle of colorimetric optical absorption, wherein samples mixed with suitable chromogenic substrates induce a color change dependent upon the analyte concentration that could then be detected by the absorbance of light in its path length. This optical detection scheme has been hybrid integrated with an acoustofluidic micromixing unit to enable uniform mixing of fluids within the device. As a proof-of-concept, we have demonstrated the real-time application of our biosensor format for the detection of potassium in whole saliva samples. The results show that our lab-on-a-chip technology could provide a useful strategy in biomedical diagnoses for rapid analyte detection towards clinical point-of-care testing applications.
\end{abstract}

Keywords: point-of-care; biosensor; hybrid integration; microfluidics; acoustofluidics; cavitation; micromixing; optical absorbance; colorimetry; salivary potassium

\section{Introduction}

Point-of-care diagnostics (POCD) for health monitoring involves the evaluation of indices from human health through tests performed outside of the clinical laboratory, typically right at the site of patient care [1-4]. While the advantages of POCD such as rapid diagnosis, operational efficiency, and costs have been well established [2], widespread implementation of this technology has not yet been achieved [5]. One of the main limitations of POCD is the requirement of unprocessed test samples drawn from patients to provide the desired sensitivity and specificity for conducting reliable assays in situ. However, most of the currently validated clinical assays are carried out using plasma, prepared from whole blood samples inside sufficiently equipped laboratory conditions. Here, we develop a biosensor platform that could be reliably used for instantaneous detection of target molecules from non-invasively obtained bodily fluid samples for real-time point-of-care testing applications.

Although whole blood assays are generally considered as the gold standard for bioanalysis, such an approach may not be convenient to implement under all circumstances. In general, high-risk 
patients under intensive care or long-term hospitalization suffering from extremely sick conditions may not always be able to bleed enough quantity of blood. Possible nerve damage during venipuncture [6] could render the procedure extremely painful, traumatic and psychologically disturbing for the patients, such that even skilled phlebotomists could face limitations to extract enough quantity of blood under such circumstances.

One strategy to overcome the trauma associated with such painful venous blood draw procedures could be to adopt a technique that is less invasive and utilizes smaller volumes, possibly through a finger prick as implemented with some of the commercially available handheld blood glucose monitors. However, a limitation with this approach is that reduced sample volumes call for greater sensitivity of the measurement setup and may also not always contain the necessary distribution of the target molecules under all circumstances. This could lead to inconsistent readings and false negative results [7]. Furthermore, common clinical problems with whole blood assays such as hemolysis or coagulation could also result in an inaccurate representation of the analyte concentration [8].

An alternate strategy would be to eliminate the necessity for whole blood, and use other test samples for carrying out the required bioanalysis. Among the bodily fluids generally used in clinical assays (shown in Figure 1), those commonly available through non-invasive extraction procedures include urine, saliva [9] and sweat [10]. While inducing sweat out of hospitalized patients is practically difficult, urinary extraction may also necessitate additional diuretics which could directly interfere with the measurands, aside from inducing other significant side effects [11]. Considering these factors, saliva presents a viable alternate to the traditional biofluids used in biochemical analyses.

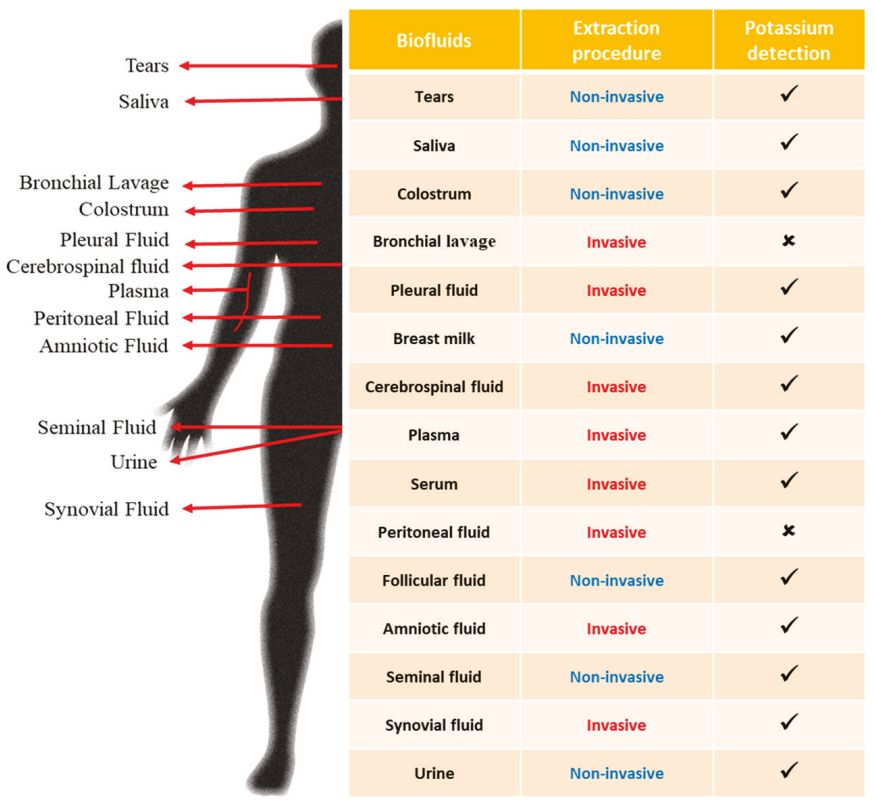

Figure 1. List of commonly available bodily fluids showing their nature of the extraction procedure and general feasibility of potassium detection from those fluids.

Saliva is considered as a filtrate of the blood representing the physiological state of the body. Given the ability of molecules from blood to diffuse into saliva, salivary diagnostics is increasingly being recognized as an equivalent to serum analysis [12]. Research studies using saliva as a bio-diagnostic fluid includes works such as evaluation towards dialysis needs in renal failure patients [9] and on-site analysis for biochemical factors [13]. The incorporation of microfluidic methods in bioassays of saliva 
has further reduced sample and reagent consumption, and has decreased the overall assay times [13]. Research studies in salivary diagnostics involving microfluidics include measurement of C-reactive protein using an fluorometric immunoassay [14], on-chip polymerase chain reaction (PCR) system for rapid fluorometric detection of genetic deletion [15], paper-based device for quantification of the nitrate concentration [16], detection of thiocyanate through surface-enhanced Raman scattering (SERS) [17], and spectrometric absorbance detection of $\mathrm{NH}_{3}$ and $\mathrm{CO}_{2}$ in saliva as a biomarker for stomach cancer [18]. In addition, most of the reported microfluidic systems for salivary diagnostics included integrated optical sensing for their bio-detection scheme [19].

In this work, we present the development of a simple, hybrid integrated optical microfluidic biosensor for rapid analysis of saliva, and we have demonstrated the application of the proposed biosensor format by detecting the presence of potassium from whole, untreated salivary samples. Inspired by the age-old concept of colorimetry published as early as the first half of the 20th century $[20,21]$, we hereby show that potassium can be detected from human saliva samples through the principle of optical absorption. A commercially available potassium detection reagent mixed with saliva samples creates turbidity based on the potassium concentration, which could then be measured through the absorption of light passing through this turbid path. We have implemented this optical sensing principle on a lab-on-a-chip platform by integrating colorimetric detection within a microfluidic system that facilitates transportation and handling of the analytes and the reagents. Homogeneous mixing of fluids inside the microfluidic system has been achieved through the integration of piezo-actuated acoustic micromixing. The results of this work show that the proposed hybrid integrated device can be applied to real-time optical biosensing using whole, unprocessed samples, which could be extremely useful for automated point-of-care testing applications.

\section{Materials and Methods}

\subsection{Development of the Integrated Optical Biosensor}

This section provides the details of the design, fabrication, integration and packaging of our hybrid integrated optical microfluidic setup. The schematic illustration of the processes is shown in Figure 2 .

\subsubsection{Device Design and Fabrication}

The biosensor (schematically shown in Figure 2A) consists of a microfluidic system with two inlets which accommodates two different types of fluids. The fluids are transported to a piezo-actuated (acoustofluidic) micromixer unit, and upon mixing are subsequently transported to the optical detection unit. The configuration of the bulk acoustofluidic micromixer unit was adopted from [22,23]. The optical detection unit consists of SU8 waveguides core integrated onto poly dimethylsiloxane (PDMS) (cladding) through the inlet and outlet waveguide channels (as indicated in Figure 2A). Convex curvatures at the end of the optical channel enable convergence of light into the fluidic channel. Two different types of optical waveguide assembly were designed. In the configuration used in the present work, the output waveguide was fabricated co-axially with the input waveguide. This configuration allowed direct coupling of the input light onto the output waveguide, as required in optical absorption measurements.

To fabricate the devices on PDMS (fabrication and integration processes schematically shown in Figure 2B), silicon master molds with patterned SU8 (100 $\mu$ m thickness) were fabricated using standard soft lithography process. Poly dimethylsiloxane (Sylgard 184, Dow Corning, Midland, MI, USA) with prepolymer to curing agent volumetric ratio of 10:1 was mixed, degassed and poured onto the silicon mold. The setup was left to cure at $70{ }^{\circ} \mathrm{C}$ for four hours, after which PDMS was peeled off the mold and diced to create the individual devices. To create optical waveguides, the PDMS devices were exposed to oxygen plasma ( $2 \mathrm{~min}, 200$ mTorr pressure, 20 cubic centimeter (ccm) flow rate, and $60 \mathrm{~W}$ Radio Frequency (RF) power), and thereafter SU8-5 (Microchem, Westborough, MA, USA) with viscosity 
$290 \mathrm{cSt}$, and refractive index $\sim 1.6$ was allowed to fill in the optical channels through capillary flow. The photoresist was cured by flood exposure to ultraviolet (UV) light $\left(12 \mathrm{~mW} / \mathrm{cm}^{2}\right)$ for $60 \mathrm{~s}$, and the devices were diced thereafter to enable fiber attachment with the SU8 waveguides.

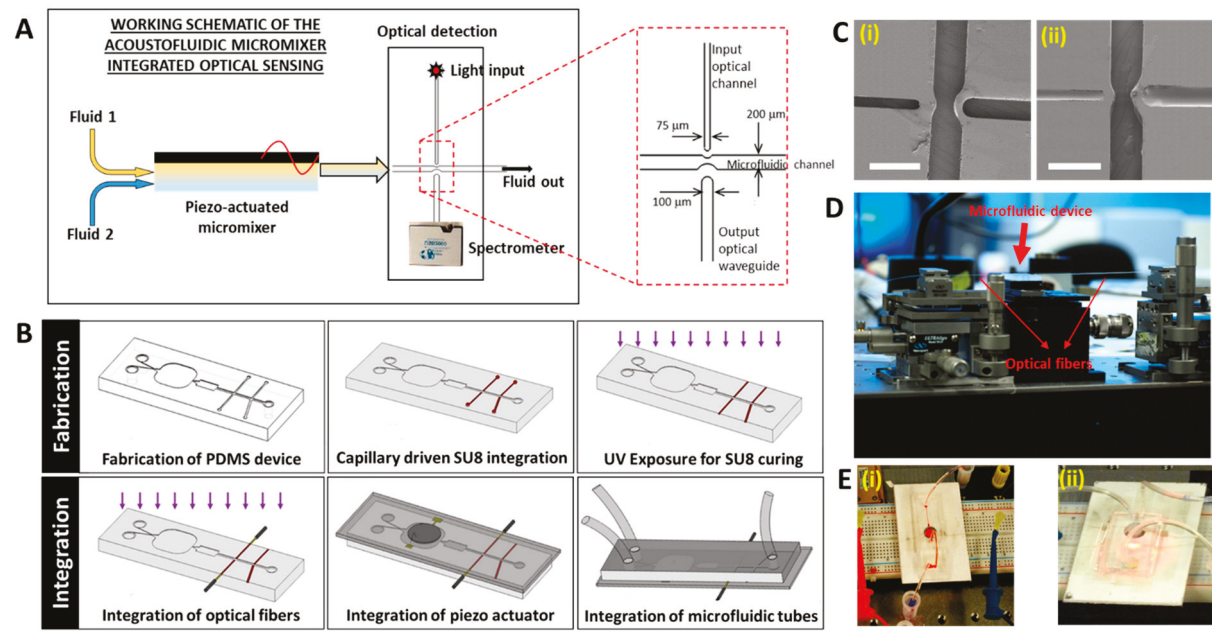

Figure 2. (A) Schematic working principle of the acoustofluidic micromixer integrated optical sensing for colorimetric detections. The liquid test specimen (fluid 1) and its corresponding chromogenic agent (fluid 2) are mixed using acoustic waves generated by a piezoacuator, and the mixture is transported into the optical detection unit, wherein the extent of color change induced due to the presence of target analyte in the sample is sensed by the absorption of light passing through the sample. (B) Schematic illustrayion of the fabrication and packaging of the hybrid integrated biosensor (C) Scanning electron microscope (SEM) images showing the microfluidic channel and the optical channels of the poly dimethylsiloxane (PDMS) device (i) before and (ii) after integration with SU8 (Scale bars represent $500 \mathrm{~m}$ ) (D) Micro-positioning setup for precise alignment and coupling of fibers with the SU8 waveguides. (E) Images of the functional integrated optical microfluidic biosensor (i) with one fluid inlet and (ii) two fluid inlets.

\subsubsection{Integration and Packaging}

Coupling of the input and the output fibers were carried out under a stereo microscope. FC connectorized tapered lens-ended fiber (OZ Optics, Ottawa, ON, Canada) which gives a spot size of $5 \mu \mathrm{m}$, was connected to a broadband laser light source (Ocean Optics, Largo, FL, USA) for optical input. The output collector fiber was SMA end connectorized and coupled to a handheld spectrometer (USB 2000, Ocean Optics, Largo, FL, USA). Fiber strippers and precision cleavers (Newport, RI, USA) were used to remove the buffer layer and cladding around the fiber. The input and the output fibers were positioned in separate five axis fiber positioners on supporting V-grooves, so that each of the fibers and the waveguides can be maneuvered and coupled appropriately for acquiring the maximum optical signal. Maximum optical light coupling into the fiber was ensured by fine-tuning the position of the fiber and by observing the maximum signal from the output fiber coupled into the spectrometer. Thereafter, UV index matching gel (NOA60, Norland International Inc., Lincoln, NE, USA) was applied at the tip of the fiber and the setup was exposed to UV for $60 \mathrm{~s}$ to bond the fiber with the SU8 waveguides.

The device was then treated with atmospheric plasma using a handheld plasma cleaner (Plasmaetch, Carson City, NV, USA) and the channels were sealed using a $100 \mu \mathrm{m}$ thick glass coverslip. Piezoceramic discs (T216-A4NO-173X, Piezo Systems Inc., Cambridge, MA, USA) used for generating the acoustic waves were attached to this glass diaphragm using silver conductive epoxy (also used as the bottom 
electrode). The top electrode was soldered to the piezoceramic. For ease of handling, the device was attached with a polycarbonate support using double-sided adhesive tape, without damaging the optical fibers. A through hole drilled on the polycarbonate prior to the attachment of the optical-microfluidic chip accomomodates the piezo actuator inside the cavity.

\subsection{Chemicals and Reagents}

Integrated optical-microfluidic characterization experiments were carried out with de-ionized (DI) water and ethanol (Sigma Aldrich, St. Louis, MO, USA). Prior to introducing fluid samples into the system, the microfluidic channels were flushed with ethanol in order to remove any contaminants or air bubbles. Initial micromixing characterization experiments were conducted by staining the working fluids (DI water or ethanol) with standard food coloring dyes. Preliminary experiments for the characterization of optical absorption with integrated micromixing were conducted using glucose enriched RPMI 1640 cell culture media (A1049101, Thermofisher, Waltham, MA, USA) as the working fluid. Herein, the glucose present in cell culture media was tested to produce an expected colorimetric reaction with Benedict's reagent prepared according to the protocol described by Cochran et al. [24]. For colorimetric salivary analysis experiments, potassium colorimetric assay kit (E-BC-K279) was procured from Cedarlane Labs (Burlington, NC, USA). Whole samples of unstimulated saliva were collected from volunteers in centrifuge tubes by a simple spitting method, and the specimens were vortexed thoroughly. Prior to the experiments, protein precipitant and chromogenic agents were prepared as described in the protocol prescribed by the manufacturer. $20 \mu \mathrm{L}$ of the saliva samples were mixed with $180 \mu \mathrm{L}$ of the protein precipitant and centrifuged at $1100 \mathrm{~g}$ for $10 \mathrm{~min}$. Thereafter, the supernatant was used in the experiments, mixed with a volumetrically consistent chromogenic agent for the colorimetric optical absorbance measurements.

\subsection{Integrated Testing and Measurement}

The piezoelectric system was driven by an external signal generator (33120A, Hewlett Packard, Palo Alto, CA, USA) and an amplifier (PCB Piezotronics, Depew, NY, USA) where a sinusoidal signal from the signal generator was amplified 20 -fold by the amplifier. Typically, the operating voltage of $100 \mathrm{~V}_{\mathrm{p}-\mathrm{p}}$ was used for piezo-actuation. For continuous flow experiments, liquids were injected at the same flow rates inside the microchannel using syringe pump (KDS-210, KD Scientific, Holliston, MA, USA). In all other cases, liquids were manually pipetted into the microfluidic channels.

The mixing efficiency was calculated based on the change in the pixel values of the respective color fluids.

Mixing efficiency $(\%)=($ Initial pixel count-final pixel count $) /($ Initial pixel count $) \times 100$

Optical signals were collected using a handheld spectrometer (USB2000, Ocean Optics, USA). The optical absorbance of the specimens measured by the spectrometer is given by the formula

$$
A_{\lambda}=-\log _{10}\left(\frac{S_{\lambda}-D_{\lambda}}{R_{\lambda}-D_{\lambda}}\right)
$$

where,

$\lambda$-Wavelength of light used

$A_{\lambda}$-Absorbance

$S_{\lambda}$-Intensity of light passing through the sample

$D_{\lambda}$-Dark intensity

$R_{\lambda}$-Intensity of light passing through a reference medium. 
The dark intensity was recorded by measuring the optical signal intensity when the light source was turned off. For potassium measurement experiments, the chromogenic agent was used as the reference medium to measure the reference intensity.

\subsection{Imaging and Statistical Analysis}

Images were recorded using an OFV-A-534-Cax video camera inbuilt with the single point Laser Doppler Vibrometer (LDV, Polytec, Detroit, MI, USA). Scanning electron microscopy (SEM, SU3500, Hitachi Hi-Technologies, Tokyo, Japan) was conducted using standard protocols for variable-pressure imaging mode $(3.0 \mathrm{kV}, 30 \mathrm{~Pa}$,) allowed SEM observations of the PDMS devices without the need for additional sample manipulation or conductive coating. The images were processed using Image (National Institute of Health, Bethesda, MD, USA), following standard protocols. All statistical comparisons were made using one- or two-way analyses of variance (ANOVA) with Tukey post-hoc comparisons (Prism; GraphPad Software, La Jolla, CA) with $p$-values $<0.05$ considered significant, and graphical data reported as means \pm standard error for at least $\mathrm{n}=3$ experiments.

\section{Results}

\subsection{Features of the Hybrid Integrated Biosensor}

The SU8 waveguide (refractive Index 1.6) integrated well onto the PDMS (refractive index $~ 1.4$ ) upon curing with UV (Figure 2C). Minor delamination of the SU8 was observed along the walls of the PDMS in some sections, however SU8 was able to guide the input light efficiently under the PDMS clad. The optical fiber assembly setup (Figure 2D) described earlier provides robust fiber attachment onto the waveguides to enable optical transmission. Also, with this arrangement of the optical microfluidic chip, the microfluidics and the micromixer modules do not interfere with the functionalities of the optical ensembles, and thus all the components of the system remain independent. Integration of on-chip spectrometer [25] was also tested to conduct direct fluorescence measurement that could be used for specific immunoassays. The fully fabricated hybrid integrated optical microfluidic device is as shown in Figure 2E.

Initial vibration analysis experiments for the mechanical characterization the piezo-actuation system was conducted using laser doppler vibrometry. The natural frequency of oscillation of the piezo-actuator was $\sim 70 \mathrm{kHz}$, much higher than the intended operating frequencies of the micromixer. As expected, the acoustic waveform generated by the piezo-actuation depends on the operating frequency. For this device configuration, acoustic cavitation inception occurred for operating frequencies greater than $\sim 800 \mathrm{~Hz}$. Upon cavitation inception, the rate of cavitation bubble growth and collapse was dependent on the operating frequency, and these parameters stabilized at $\sim 3000 \mathrm{~Hz}$, beyond which no significant differences were observed in the life-cycle or the behavior of the cavitation bubbles. On the other hand, at lower operating frequencies $(1 \sim 100 \mathrm{~Hz})$, the piezo-actuation principle could be used for transporting fluid across the channels through valveless micropumping [24]. Slight modifications to the existing design enable the feasibility of integrating other types of optical detection techniques, namely fluorescence or evanescence for a correspondingly appropriate type of integrated microfluidic biosensing [26].

\subsection{Acoustically Induced Cavitation Enables Rapid Micromixing}

Effective mixing of fluids is important in several applications including chemical synthesis, biochemical reactions, and clinical diagnosis. As with any biochemical assay, for accurate colorimetric quantification sufficient mixing of fluids is important in order to homogenize the specimens with the corresponding reagents [27]. However, one of the limitations with microfluidics is the laminarity of flow in the device, which renders turbulent mixing difficult. Here, by incorporating a piezo-actuated acoustic micromixer within the microfluidic design, we experimentally demonstrated the feasibility of controlled mixing of two non-reacting liquids through the inception of cavitation bubbles. 
For convenience and ease of visualization, ethanol was stained blue and water was stained with red dye in all the experiments. Under continuous flow, the flow of liquids inside the microfluidic channel was extremely laminar (calculated Reynolds number $\sim 5$ ). The mixing ability of fluids under flow was tested in different conditions, and no mixing was observed even for similar liquids of very high miscibility (blue ethanol colorless ethanol) in both with or without the actuation of the micromixer (Figure 3A(i)). For dissimilar liquids (water-ethanol), instantaneous diffusion mixing was also negligible for both continuous flow and 'static' (no flow) conditions. Once the flow of liquid in the channel was stopped, actuation of the micromixer at $1000 \mathrm{~Hz}$ induced the formation of cavitation bubble in water (Figure 3A(ii)). We believe that PDMS substrates, being gas permeable facilitates the generation of bubbles, even for such low actuation frequencies, which in this case was useful for fluid mixing. Thereafter, reduction of the operating frequency to $800 \mathrm{~Hz}$ retarded the growth of the cavitation bubble, and at the same time creating micro-vortices in the fluids to initiate mixing. The real-time sequence of cavitation assisted fluid mixing is as shown in Figure 3B. Mixing of the fluids was instantaneous upon the inception of the cavitation bubble. Increasing the actuation frequency decreased the time taken for cavitation bubble inception (Figure 3C). However, with increased frequency, the growth of the cavitation bubble was also rapid and difficult to control precisely. Therefore, to create steady cavitation, all micromixing experiments were conducted at an actuation frequency of $1000 \mathrm{~Hz}$ to initiate the cavitation bubble, and thereafter the operating frequency was reduced to $800 \mathrm{~Hz}$ to allow fluid mixing. The actuation frequency of our bulk acoustofluidic micromixer is around 100 fold lower than micromixing actuation using surface acoustic waves [27]. Lower actuation frequency could be useful for handling samples that contain living cells, as high frequency could induce undesired effects such as cell proliferation, differentiation, lysis etc. [28].
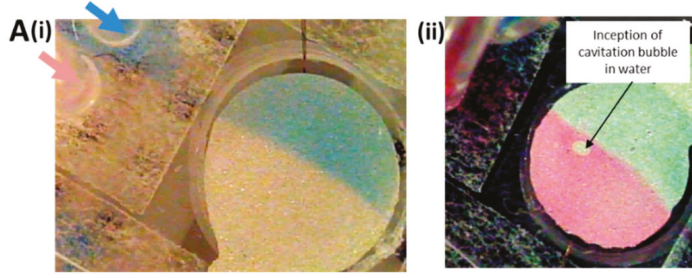

B
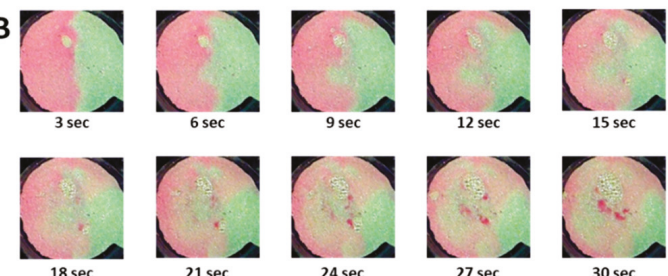
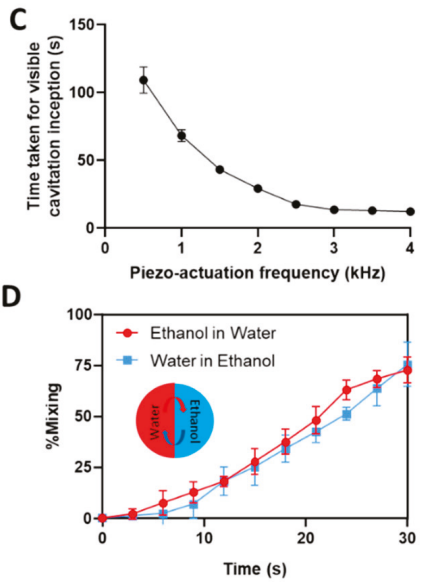

Figure 3. (A) (i) Image of the microfluidic channel with two inlets allowing laminar flow of two shades of ethanol (colorless and blue) without mixing when introduced from either of the inlets. (ii) Image showing the inception of cavitation bubble in water (red) to initiate the mixing of fluids (B) Image sequence showing the cavitation enabled mixing of water and ethanol inside the microfluidic channel. (C) Variation of the time taken for the inception of a visible cavitation bubble with respect to the piezo actuation frequency. Cavitation inception time was consistent (within 3\% variation) for actuation frequencies greater than $1 \mathrm{kHz}$. (D) variation in the fraction of the respective fluids mixed inside the microchannel with respect to time. To enable this fluid mixing, caviation was initiated at $1000 \mathrm{~Hz}$ actuation frequency, and the working frequency was reduced to $800 \mathrm{~Hz}$, so as to retard the growth of the cavitation bubble. 
The mixing efficiency was studied by measuring the concentration of red pixels in the section of the microfluidic channel initially containing ethanol (blue) and the concentration of blue pixels in the microchannel section initially containing water. Within $30 \mathrm{~s}$, a mixing efficiency of more than $75 \%$ over a volume of $\sim 3 \mu \mathrm{L}$ of the fluid (Figure 3D). This is comparable to some of the previously published results. For example, Liu et al. [29] have reported cavitation assisted mixing of $22 \mu \mathrm{L}$ of the fluids in $\sim 105 \mathrm{~s}$, and for the system proposed by [30], the mixing efficiency is governed by the flow rate which is $8 \mu \mathrm{L} / \mathrm{min}$. Both these values are close to what we observed, thus corroborating with the efficiency achieved through our micromixing technique. Continued operation of the micromixer produces agglomeration of non-covalently bound particulates from the coloring dye which interfered with mixing. However, for proof-of-concept preliminary demonstration of fluid mixing, our experiments show that efficient mixing of small fluid sample volumes can be achieved by integrating the piezo-actuated acoustic micromixer that generates micro-vortices through the controlled induction of cavitation bubbles.

\subsection{Demonstration of On-Chip Optical Absorption}

One of the major drawbacks of absorbance-based detection in microfluidics is that it is considered to decrease in the optical path length with reduced usage of the sample volumes, which could directly affect the sensitivity of the system [31]. Therefore, in order to ascertain whether our device configuration was sensitive enough to support real-time optical absorption measurements, we conducted a preliminary non-calibrated colorimetric assay. For this proof-of-concept experiment, we demonstrated the detection of glucose [32] in cell culture media, based on its reaction with Benedict's reagent.

It is well known that glucose, a reducing sugar, induces the conversion of cupric ions $\left(\mathrm{Cu}^{2+}\right)$ to cuprous form $\left(\mathrm{Cu}^{+}\right)$in the Benedict's reagent [33]. This reaction brings about a change in color of the solution mixture, with the coloration being dependent upon the amount of glucose present. To firstly verify this principle, glucose-enriched media (hereafter referred to as glucose solution) was diluted to varying concentrations and mixed with the Benedict's reagent. The color changes in the mixture samples are as shown in Figure 4A. The color observed for the reactant mixture for undiluted glucose solution is indicated with the arrow. Upon heating the mixture, the color of those reactants changed to dark red as expected.

Before implementing this colorimetric reaction inside the integrated optical microfluidic setup, baseline optical absorption values at $635 \mathrm{~nm}$ wavelength were recorded by passing the glucose solution and the Benedict's reagent individually through the microfluidic channel. Thereafter, to initiate the reaction, the liquids were pipetted simultaneously into the microfluidic chamber, and the piezoacoustic micromixer was actuated. To the naked eye, the liquids initially appeared colorless upon entering the micromixing chamber (Figure $4 \mathrm{~B}$ at $\mathrm{t}=0$ ). However, with continued piezo-actuation, mixing of the fluids causes an increase in the turbidity of the reaction mixture (Figure 4B).

The liquids were allowed to mix for $60 \mathrm{~s}$, after which the piezo-actuation frequency was increased to drive the liquids under supercavitation [22] through the optical detection zone and finally out of the channels. Upon image processing, the color of this reaction mixture inside the microfluidic chamber (Figure 4B) and the color of the ejected reaction mixture (indicated in Figure 4C) was observed to be very close to the externally prepared reaction mixture indicated in Figure $4 \mathrm{~A}$, The results for the measurement of the colorimetric optical absorption is presented in Figure 4D. As expected, at $635 \mathrm{~nm}$ wavelength, the optical absorption of the blue colored Benedict's reagent was higher than the phenol-red tagged glucose solution (Figure 4D(i)). The initial randomness in the optical absorbance signal for the reaction mixture (Figure $4 \mathrm{D}(\mathrm{ii})$ ), is we believe, noise due to the flow of cavitation bubbles in the reaction mixture. The mean optical absorption of the reaction mixture was significantly higher $(p<0.001)$ than the both individual liquids, presumably due to the increased turbidity obtained during the reaction (Figure $4 \mathrm{E}$ ). The optical absorbance value of reactants mixed inside the microfluidic channels was very close ( $<5 \%$ variation) to that of the reaction mixture externally prepared by manually vortexing the reacting fluids, thereby further confirming the homogeneity of the micromixing process. The experiment was repeated 3 times, and the results were found to be consistent. Thus, the results 
of this experiment demonstrates the implementation of optical absorption measurement, with our micromixing-enabled optical sensing setup.
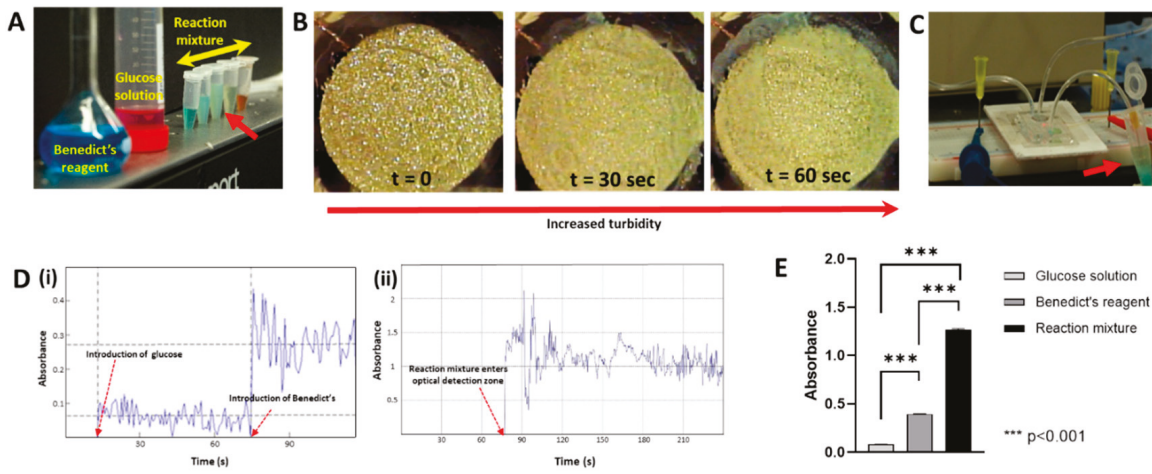

Figure 4. Implementation of Benedict's reaction inside the optical microfluidic device for colorimetric detection of glucose in the presence of cell culture media. (A) Change in the color of the specimens for various concentrations of the glucose solutions, with the diluted samples showing shades of blue color. Reaction mixture upon heating turned to brick red color as expected. Arrow indicates the color obtained with undiluted glucose. (B) Variation, at different time points, in the turbidity of the reacting fluids (glucose solution and Benedict's solution) upon piezo actuated mixing inside the microfluidic channel. (C) Reaction mixture ejected out of the microfluidic channel, indicating the same coloration as indicated in panel-A. (D) Real-time absorbance measurements at $635 \mathrm{~nm}$ wavelength of (i) glucose solution and the Benedict's reagent and (ii) Reaction mixture upon entering the optical detection zone. (E) Comparison of the optical absorption values of the working fluids. The reaction mixture shows a significantly higher absorbance $(p<0.001)$ than both the glucose solution and the Benedict's solution, due to the turbidity created upon mixing, thereby validating the colorimetric detection principle.

\subsection{Colorimetric Detection of Salivary Potassium}

With the colorimetric detection feasibility well established through the proof-of-concept glucose detection experiments, we further explored the possibility of implementing our microfluidics-integrated optical detection platform for clinically relevant real-time biosensing applications. Potassium monitoring and level correction is a routine but critical procedure carried out at the hospitals for patients undergoing cardiac, renal or neurological related diagnoses. Altered potassium levels in the body under hyperkalemia or hypokalemia may cause arrhythmia, abnormal blood pressure, cramping, twitching or paralysis of muscles leading to the development of abnormal cardiac rhythms $[34,35]$. Since whole blood assays currently adopted in hospitals for potassium monitoring is painful and sometimes unsuccessful, we reasoned that salivary diagnostics of potassium could be far less traumatic and could generate rapid results when performed in a point-of-care setting. To validate this hypothesis, we tested whole saliva samples through colorimetric optical absorption, for the measurement of absolute salivary potassium concentration.

The absorption measurements were conducted at $450 \mathrm{~nm}$ wavelength, as prescribed by the manufacturers of the potassium detection kit. Initial control experiments were conducted to measure the optical absorbance values of test samples with known potassium concentration. Unstimulated saliva samples were collected from random volunteers on the North Carolina A \& T State University campus. Samples were collected from 3 volunteers above the age of 65 years, 6 volunteers in the age group of 35-65 years, and 5 student volunteers under the age of 35 years. The results of our colorimetric potassium concentration measurement from the collected saliva samples are presented in Figure 5A. The overall estimated mean concentration of salivary potassium was found to be well within the range of typical salivary potassium levels as reported earlier [36]. The mean unstimulated potassium 
concentration with people between the age of 13 and 35 has been reported to be $\sim 21 \mathrm{mmol} / \mathrm{L}$ [37]. These observations match the data from our experimental results as well within $5 \%$ error, thereby validating our approach for a colorimetric optical absorbance-based salivary analysis method used herein.

However, contradicting observations have been made in literature regarding exact salivary potassium concentrations for patients with health conditions. We reasoned that there are several factors that could influence the salivary electrolyte concentrations, and a larger data set is required to make any robust comparison and inference using our device. However, the intent of the present work is far from delving into detailed biochemical analyses of saliva under different conditions. The objective of this work is to demonstrate the application of colorimetric sensing using microfluidic mixing for optical sensing. Therefore, in order to validate our optical measurements, we measured the optical absorbance of three random saliva samples treated with the chromogenic agents on-chip, and compared the results to the optical absorption of those samples measured externally using a standard spectrophotometer (Cary 6000i UV/Vis/NIR, Agilent, Santa Clara, CA, USA). The results shown in Figure 5B demonstrate no significant difference between the absorbance values measured through these two techniques, thereby validating our on-chip analysis approach. The time-dependent on-chip absorbance measurements and the absorbance spectrum obtained using the spectrophotometer for one of the samples is presented in Supplementary Figure S1A.

A

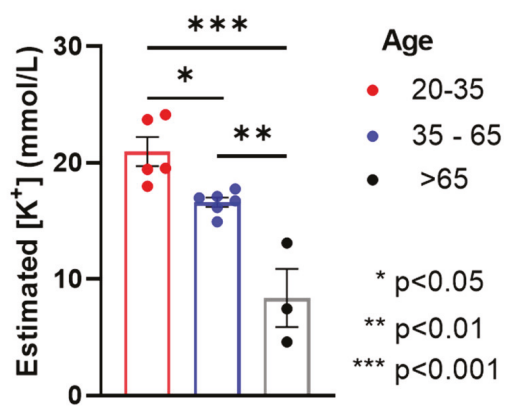

B

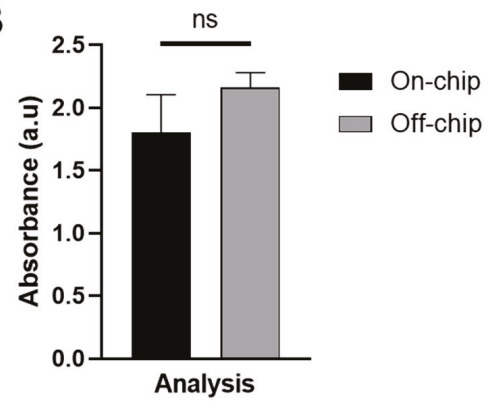

Figure 5. Results of the colorimetric optical absorbance detection of potassium from unstimulated whole saliva. (A) Measurement of estimated potassium concentration among people (15 volunteers) of different age groups indicating that older people ( $>65 \mathrm{y}$ age) show a significantly lesser $(p<0.01)$ salivary potassium content than people in the age group of 35-65; and also significantly reduced potassium than people in 20-35 age group. In general people above the age of 35 show significantly lesser $(p<0.05)$ potassium than people under the age of 35. (B) Comparison of the optical absorbance values of salivary samples mixed with chromogenic agent measured on chip and off-chip (using an ultraviolet/visible (UV/Vis) spectrometer) shows no significant difference ( $p=0.2643$ with paired t-test) for the samples analyzed through the two measurement techniques.

Thus, the results of our work show that the colorimetric optical absorption based biosensing principle with integrated micromixing can be readily applied to the lab-on-a-chip format for real-time analysis of metabolites from saliva to generate diagnostic results with a quick turn-around.

\section{Discussion}

Salivary diagnostics is being increasingly touted as a suitable alternative to whole blood assays. However, the study of salivary functions could also be challenging because of the high physiological variability of saliva in comparison with other bodily fluids [38]. However, given that the saliva samples can be obtained non-invasively, patients are much more likely to cooperate for frequent extraction of saliva, for performing assays regularly. We also observed this trend among the volunteers, who were unanimously relieved with the painless approach of collecting saliva samples for our experiments. Nevertheless, to exploit this convenience of salivary analysis, it is also equally important to develop a 
suitable automated point-of-care testing methodology that would enable rapid diagnostics to reduce the time and effort normally put into laboratory-based assays. The cumulative results of our present work demonstrates that the hybrid integrated optical biosensing principle proposed herein could be readily applied to potassium measurement from whole saliva samples for real-time point-of-care testing applications based on the principle of colorimetry.

The colorimetric optical absorption-based detection principle presented here is simple and can be easily extended to any other fluid sample. While the results of traditional point-of-care colorimetric assays reported to the naked eye (such as pregnancy tests or blood glucose tests), integrating smart-phones [39] for automated testings have lately been gaining huge popularity. But, the implementation of the colorimetric technique would not be effective unless uniform, controlled mixing of samples with the appropriate chromogenic agents is achieved. The acoustofluidic actuator thus lends a major advantage to this device for achieving the required homogenous sample mixing. The bulk acoustic wave micromixer configuration described in this work is simple and cost-effective to fabricate. For the device setup presented here, the ease of operation and feasibility of seamless hybrid integration with other complementary modules of the biosensor platform lend advantages to the bulk acoustic wave micromixer over the popularly used surface acoustic wave micromixing formats. However, mixing efficiency could be further improved by adopting simple modifications to the existing setup, such as incorporation of passive microfluidic elements, surface treatment resulting in increased hydrophobicity [40], and using acoustic index matching glues to improve piezoacoustic energy transfer. Our initial experiments showed that the cavitation-based acoustofluidic micromixing can be used with a wide variety of fluids with different physical properties such as miscibility, viscosities, vapor pressures etc. (Supplementary Figure S1B). While the present work focused only on cavitation-enabled fluid mixing, it was also observed during the initial characterization experiments that the cavitation phenomenon also induced other physical effects in the fluids. For example, it was possible to take advantage of the energy released from collapse of the cavitation bubbles for cleaning the surface of the microfluidic channels to get rid of non-specifically bound adherent particles [26]. This functionality could be extremely useful for conducting biological assays repeatedly without cross-contamination inside the microfluidic channels.

However, there are a few shortcomings to the present approach. Firstly, the device format in its present state does not enable the realization of cheap, disposable biosensors. But, since the device is sterilizable for repeated use, integrating light sources and sensor units on-chip along with suitable optoelectronic packaging could enable further help with the miniaturization of this device for developing a more cost-effective version. Secondly, optical absorption-based detection may hit the limits when conducting assays for measuring extremely low analyte concentrations in the samples. Despite the relatively poor sensitivity of microfluidic absorbance detection compared to fluorescence, the principle of working of optical absorption and its instrumentation simplicity gives it an advantage in applications requiring point-of-use analysis. Also, wherever applicable, it is possible to carry out a comprehensive study of time-dependent biochemical interactions using the optical absorption method. One can also take confidence from the fact that a number of absorbance-based microfluidic point-of-care products are commercially available [41]. Our device design also allows for implementing more than one optical sensing principle on the same platform. In an alternate waveguide arrangement to that used in the present work, the output waveguide was designed at an angle with respect to the inlet waveguide so that light is not directly coupled onto the output waveguide. This configuration, although not used in this work, could be useful for sensitive low absorption measurements or fluorescence-based optical detections where specific emission wavelengths are sensed spectroscopically without being saturated by the excitation wavelengths. Thus, overall our present work clearly demonstrates the feasibility of developing integrated microfluidic optical detection systems for rapid biosensing and real-time diagnostics, which could be incorporated into the next generation point-of-care devices. 


\section{Conclusions}

Saliva is a non-invasive biofluid which is easy to collect, transport, and store. Because of its accessibility and connection to systemic diseases, saliva is one of the best candidates for the advancement of point-of-care medicine, where individuals are able to easily monitor their health status by integrating the salivary diagnostics with suitable biosensing systems. Our present work on the development of a hybrid integrated optical detection based biosensor with integrated micromixing further asserts that colorimetric salivary analysis can be conducted in real time in a dependable, noninvasive, simple, and rapid manner using whole, unprocessed saliva samples with the appropriate chromogenic agents, to screen for any required minerals and metabolite values Thus, this approach could come in handy for real-time point-of-care screening applications for monitoring high-risk hospitalized patients.

Supplementary Materials: The following are available online at http:/www.mdpi.com/2079-6374/9/2/73/s1: Figure S1: (A). (i) Time varying optical absorbance of a saliva sample mixed with chromogenic agent measured on-chip using the hybrid integrated device. The mean optical absorbance value was 2.36 (ii) Optical absorbance measurement of the same sample measured using a UV/Vis spectrophotometer showing the absorbance value at $450 \mathrm{~nm} \sim 2$.4. (B). Preparation of water in oil emulsion: Finer dispersion of water (blue) in the colorless oil medium throughout the chamber is observed with the actuation of the micromixer. Much longer micromixing time ( 600 s) was required to achieve this, Figure S2: Enlarged versions of Figure 2E, Figure 4A,C.

Author Contributions: A.C. and M.P. designed the research. V.S. and A.C. conducted the literature review. V.S. T.C., S.M. and A.C. conducted the experiments. A.C., M.P. and S.K. analyzed the results. V.S., A.C., T.C., S.M., S.K. and M.P. prepared the manuscript.

Funding: This research was funded by grant provided to A.C. by the College of Engineering (CoE), North Carolina A\&T State University (NC A\&T SU), and Discovery grant provided to MP by the Natural Sciences and Engineering Research Council of Canada (NSERC). The APC was funded by grant from the CoE, NC A\&T SU, provided to AC.

Acknowledgments: The authors would like to thank Shyam Aravamudhan, North Carolina Agricultural and Technical State University-Joint School of Nanoscience and Nanoengineering, for generously donating the potassium detection kit. The authors would also like to thank Taylor Mabe for his assistance with the experiments.

Conflicts of Interest: The authors declare no conflict of interest.

\section{References}

1. Vashist, S. Point-of-care diagnostics: Recent advances and trends. Biosensors 2017, 7, 62. [CrossRef] [PubMed]

2. Patel, K.; Suh-Lailam, B.B. Implementation of point-of-care testing in a pediatric healthcare setting. Crit. Rev. Clin. Lab. Sci. 2019, 6, 1-8. [CrossRef] [PubMed]

3. Liang, C.; Liu, Y.; Niu, A.; Liu, C.; Li, J.; Ning, D. Smartphone-App based point-of-care testing for myocardial infarction biomarker cTnI using an autonomous capillary microfluidic chip with self-aligned on-chip focusing (SOF) lenses. Lab Chip 2019, 19, 1797-1807. [CrossRef] [PubMed]

4. Li, Z.; Liu, H.; He, X.; Xu, F.; Li, F. Pen-on-paper strategies for point-of-care testing of human health. TrAC Trends Anal. Chem. 2018, 108, 50-64. [CrossRef]

5. Vashist, S.K.; Luppa, P.B.; Yeo, L.Y.; Ozcan, A.; Luong, J.H. Emerging technologies for next-generation point-of-care testing. Trends Biotechnol. 2015, 33, 692-705. [CrossRef] [PubMed]

6. Oven, S.D.; Johnson, J.D. Radial nerve injury after venipuncture. J. Hand Microsurgery 2017, 9, 043-044.

7. Bond, M.M.; Richards-Kortum, R.R. Drop-to-drop variation in the cellular components of fingerprick blood: Implications for point-of-care diagnostic development. Am. J. Clin. Pathol. 2015, 144, 885-894. [CrossRef] [PubMed]

8. Ismail, A.; Shingler, W.; Seneviratne, J.; Burrows, G. In vitro and in vivo haemolysis and potassium measurement. BMJ 2005, 330, 949. [CrossRef]

9. Hashemi, J.; Hesari, Z.; Golshan, A.R. Evaluation of calcium, phosphorus and potassium in saliva and their relationship to blood biochemical factors in hemodialysis patients. Tehran Univ. Med. J. 2017, 75, 65-71.

10. Koh, A.; Kang, D.; Xue, Y.; Lee, S.; Pielak, R.M.; Kim, J.; Hwang, T.; Min, S.; Banks, A.; Bastien, P.; et al. A soft, wearable microfluidic device for the capture, storage, and colorimetric sensing of sweat. Sci. Transl. Med. 2016, 8, 366ra165. [CrossRef]

11. Bel'skaya, L.V.; Kosenok, V.K.; Sarf, E.A. Chronophysiological features of the normal mineral composition of human saliva. Arch. Oral Biol. 2017, 82, 286-292. [CrossRef] [PubMed] 
12. Bagalad, B.S.; Mohankumar, K.P.; Madhushankari, G.S.; Donoghue, M.; Kuberappa, P.H. Diagnostic accuracy of salivary creatinine, urea, and potassium levels to assess dialysis need in renal failure patients. Dent. Res. J. 2017, 14, 13. [CrossRef]

13. Javaid, M.A.; Ahmed, A.S.; Durand, R.; Tran, S.D. Saliva as a diagnostic tool for oral and systemic diseases. J. Oral Biol. Craniofac. Res. 2016, 6, 67-76. [CrossRef] [PubMed]

14. Christodoulides, N.; Mohanty, S.; Miller, C.S.; Langub, M.C.; Floriano, P.N.; Dharshan, P.; Ali, M.F.; Bernard, B.; Romanovicz, D.; Anslyn, E.; et al. Application of microchip assay system for the measurement of C-reactive protein in human saliva. Lab Chip 2005, 5, 261-269. [CrossRef] [PubMed]

15. Lien, K.Y.; Liu, C.J.; Kuo, P.L.; Lee, G.B. Microfluidic system for detection of $\alpha$-thalassemia-1 deletion using saliva samples. Anal. Chem. 2009, 81, 4502-4509. [CrossRef] [PubMed]

16. Bhakta, S.A.; Borba, R.; Taba, M., Jr.; Garcia, C.D.; Carrilho, E. Determination of nitrite in saliva using microfluidic paper-based analytical devices. Anal. Chim. Acta 2014, 809, 117-122. [CrossRef] [PubMed]

17. Wu, L.; Wang, Z.; Zong, S.; Cui, Y. Rapid and reproducible analysis of thiocyanate in real human serum and saliva using a droplet SERS-microfluidic chip. Biosens. Bioelectron. 2014, 62, 13-18. [CrossRef] [PubMed]

18. Zilberman, Y.; Sonkusale, S.R. Microfluidic optoelectronic sensor for salivary diagnostics of stomach cancer. Biosens. Bioelectron. 2015, 67, 465-471. [CrossRef]

19. Kumar, S.; Kumar, S.; Ali, M.A.; Anand, P.; Agrawal, V.V.; John, R.; Maji, S.; Malhotra, B.D. Microfluidic-integrated biosensors: Prospects for point-of-care diagnostics. Biotechnol. J. 2013, 8, 1267-1279. [CrossRef]

20. Abul-Fadl, M.A.M. Colorimetric determination of potassium by Folin-Ciocalteu phenol reagent. Biochem. J. 1949, 44, 282. [CrossRef]

21. Caley, E.R. The rapid colorimetric estimation of potassium. J. Am. Chem. Soc. 1931, 53, 539-545. [CrossRef]

22. Chandrasekaran, A.; Packirisamy, M. Experimental investigation of cavitation behavior in valveless micropumps. J. Micromech. Microeng. 2012, 22, 125019. [CrossRef]

23. Chandrasekaran, A.; Packirisamy, M. A study of cavitating and non-cavitating performances of valveless micropump through dynamic measurement of chamber pressure. J. Micromech. Microeng. 2015, 25, 035006. [CrossRef]

24. Cochran, B.; Lunday, D.; Miskevich, F. Kinetic analysis of amylase using quantitative Benedict's and iodine starch reagents. J. Chem. Educ. 2008, 85, 401. [CrossRef]

25. Chandrasekaran, A.; Packirisamy, M. Integrated microfluidic biophotonic chip for laser induced fluorescence detection. Biomed. Microdevices 2010, 12, 923-933. [CrossRef] [PubMed]

26. Chandrasekaran, A. Cavitation Assisted and Enhanced Valveless Micropumping Integrated Optical Detection based Micro-Total Analysis System (mTAS): Design, Modeling, Fabrication and Testing. Ph.D. Thesis, Concordia University, Montreal, QC, Canada, 2011.

27. Ozcelik, A.; Ahmed, D.; Xie, Y.; Nama, N.; Qu, Z.; Nawaz, A.A.; Huang, T.J. An acoustofluidic micromixer via bubble inception and cavitation from microchannel sidewalls. Anal. Chem. 2014, 86, 5083-5088. [CrossRef]

28. Rubin, D.; Anderton, N.; Smalberger, C.; Polliack, J.; Nathan, M.; Postema, M. On the Behaviour of Living Cells under the Influence of Ultrasound. Fluids 2018, 3, 82. [CrossRef]

29. Liu, R.H.; Yang, J.; Pindera, M.Z.; Athavale, M.; Grodzinski, P. Bubble-induced acoustic micromixing. Lab Chip 2002, 2, 151-157. [CrossRef]

30. Ahmed, D.; Mao, X.; Shi, J.; Juluri, B.K.; Huang, T.J. A millisecond micromixer via single-bubble-based acoustic streaming. Lab Chip 2009, 9, 2738-2741. [CrossRef]

31. Myers, F.B.; Lee, L.P. Innovations in optical microfluidic technologies for point-of-care diagnostics. Lab Chip 2008, 8, 2015-2031. [CrossRef]

32. Park, J.; Ha, B.H.; Destgeer, G.; Jung, J.H.; Sung, H.J. An acoustothermal heater for paper microfluidics towards point-of-care glucose detection. Phys Procedia 2015, 70, 46-49. [CrossRef]

33. Benedict, S.R. A reagent for the detection of reducing sugars. J. Biol. Chem. 1909, 5, 485-487.

34. Newmark, S.R.; Dluhy, R.G. Hyperkalemia and hypokalemia. JAMA 1975, 231, 631-633. [CrossRef] [PubMed]

35. Gennari, F.J. Disorders of potassium homeostasis: Hypokalemia and hyperkalemia. Crit. Care Clin. 2002, 18, 273-288. [CrossRef]

36. Feron, G. Unstimulated saliva: Background noise in taste molecules. J. Texture Stud. 2019, 50, 6-18. [CrossRef] [PubMed] 
37. Ben-Aryeh, H.; Fisher, M.; Szargel, R.; Laufer, D. Composition of whole unstimulated saliva of healthy children: Changes with age. Arch. Oral Biol. 1990, 35, 929-931. [CrossRef]

38. Lasisi, T.J.; Fasanmade, A.A. Comparative analysis of salivary glucose and electrolytes in diabetic individuals with periodontitis. Ann. Ib. Postgrad. Med. 2012, 10, 25-30.

39. Kim, S.D.; Koo, Y.; Yun, Y. A smartphone-based automatic measurement method for colorimetric pH detection using a color adaptation algorithm. Sensors 2017, 17, 1604. [CrossRef]

40. Ward, K.; Fan, Z.H. Mixing in microfluidic devices and enhancement methods. J. Micromech. Microeng. 2015, 25, 094001. [CrossRef]

41. Pires, N.; Dong, T.; Hanke, U.; Hoivik, N. Recent developments in optial detection technologies in lab-on-a-chip devices for biosensing applications. Sensors 2014, 14, 15458-15479. [CrossRef]

(C) 2019 by the authors. Licensee MDPI, Basel, Switzerland. This article is an open access article distributed under the terms and conditions of the Creative Commons Attribution (CC BY) license (http://creativecommons.org/licenses/by/4.0/). 
Article

\title{
Analytical Solution of the Time-Dependent Microfluidic Poiseuille Flow in Rectangular Channel Cross-Sections and Its Numerical Implementation in Microsoft Excel
}

\author{
Patrick Risch, Dorothea Helmer, Frederik Kotz and Bastian E. Rapp * \\ Department of Microsystems Engineering (IMTEK), Laboratory of Process Technology | NeptunLab, \\ Albert-Ludwigs University of Freiburg, 79085 Freiburg im Breisgau, Germany; Patrick.Risch@imtek.de (P.R.); \\ Dorothea.Helmer@imtek.de (D.H.); Frederik.Kotz@imtek.de (F.K.) \\ * Correspondence: Bastian.Rapp@imtek.de
}

Received: 15 April 2019; Accepted: 22 May 2019; Published: 24 May 2019

\begin{abstract}
We recently demonstrated that the Navier-Stokes equation for pressure-driven laminar (Poiseuille) flow can be solved in any channel cross-section using a finite difference scheme implemented in a spreadsheet analysis tool such as Microsoft Excel. We also showed that implementing different boundary conditions (slip, no-slip) is straight-forward. The results obtained in such calculations only deviated by a few percent from the (exact) analytical solution. In this paper we demonstrate that these approaches extend to cases where time-dependency is of importance, e.g., during initiation or after removal of the driving pressure. As will be shown, the developed spread-sheet can be used conveniently for almost any cross-section for which analytical solutions are close-to-impossible to obtain. We believe that providing researchers with convenient tools to derive solutions to complex flow problems in a fast and intuitive way will significantly enhance the understanding of the flow conditions as well as mass and heat transfer kinetics in microfluidic systems.
\end{abstract}

Keywords: microfluidics; numerical techniques; Microsoft Excel; finite difference method; Navier-Stokes; time dependent flow; dynamic flow; initiation of flow; Poiseuille flow

\section{Introduction}

The fundamental physics of flows in microchannels are pivotal for the precise control of dynamic effects underlying transport phenomena such as momentum, mass or heat transfer and ultimately define the behavior or the system at hand for a practical application. As an example, establishing the correct flow physics is crucial for modeling and understanding the transport kinetics of an analyte from the bulk of the flow to the surface of, e.g., a biosensor [1]. In particular, the response dynamics of a biosensor strongly depends on the diffusion kinetics of the analyte within the bulk of the flow of the biosensor. The formation of the Nernst diffusion layer is the limiting factor that defines the transport dynamics and thus the dynamic response of the biosensor [2]. This is particularly important during the initiation of the flow and in transitions of the flow such as, e.g., at the early stage of an experiment. These are tightly interlinked with the fluid mechanics of the system and thus a detailed understanding, specifically of transient effects in the bulk fluid flow, is pivotal for a correct system assessment [3]. In many respects, the fluid physics of microfluidics are straight-forward. Given the low Reynolds number flows commonly encounter in microfluidics, effects such as turbulence are rarely relevant in microfluidic flows [4].

However, given the fact that the underlying equation of fluid dynamics, i.e., the Navier-Stokes equation, is very difficult to solve even in (seemingly) simple channel geometries and flow conditions, researchers commonly refer to numerical methods to model the flow physics [5]. However, correctly 
applying a complex numerical modeling or solver software package is usually beyond the scope of application-driven research in microfluidics and adjacent fields.

We have recently demonstrated, that the simplified Navier-Stokes equation for laminar flow can be conveniently solved in a spreadsheet analysis software such as Microsoft Excel [6,7]. Spreadsheet tools are convenient platforms for implementing schemes in an intuitive and documentable manner. Software packages such as Microsoft Excel are widely available, and researchers are used to working with these packages from their daily work routine. Thus, applying a scheme which is implemented on this platform comes with a significantly lower barrier than adopting a complex and costly numerical solver package.

In our two most recent contributions, we demonstrated that the Poiseuille equation, a simplified version of the Navier-Stokes equation for stationary laminar flows, can be implemented in a wide range of channel geometries. For geometries for which analytical solutions can be obtained, the numerical results from the spreadsheet deviate only by a few percent from the analytical solutions [6]. We also demonstrated that the spreadsheet can be used to implement different boundary conditions besides the commonly employed no-slip (Dirichlet) boundary condition such as, e.g., Neumann-type boundary conditions which occur on slip surfaces as well as on open channel geometries [7]. These solutions are close-to-exact to the analytical solutions, which again, can only be derived for very simple channel geometries, usually with a high degree of symmetry such as, e.g., in circular channel (Hagen-Poiseuille) flows.

However, all of these solutions were derived for stationary flow scenarios, i.e., flows for which the time-dependency of the Poiseuille equation is ignored. This case is applicable for applications where the flow is assumed to be in steady-state and flow acceleration (i.e., during initiation of the flow by applying a pressure drop), as well as flow retardation (i.e., during stopping of the flow by removing the driving pressure drop) are not taken into account. However, there are many applications where time-dependence needs to be considered, especially in applications where the momentum diffusion is overlaid by, e.g., mass diffusion. In this paper we will show that the spreadsheets can be designed to reflect time dependency, allowing the study of transient effects during flow initiation and retardation, as well as intermediate changes in the driving pressure drop which modifies the flow conditions.

\section{Numerical Scheme}

\subsection{Navier-Stokes Equation for Time-Dependent Flow}

In comparison to the stationary version of the Navier-Stokes equation used in [6], we now have to consider time-dependency. Neglecting volume forces, the Navier-Stokes equation simplifies to

$$
\rho \frac{\partial \vec{v}}{\partial t}=-\vec{\nabla} p+\eta \Delta \vec{v}
$$

which includes pressure contribution $(\vec{\nabla} p)$, momentum diffusion $(\eta \Delta \vec{v})$, as well as time-dependency $\left(\frac{\partial \vec{v}}{\partial t}\right)$. Again, we consider parallel flow which reduces the dependent variable $\vec{v}$ (a vector) to only the contribution $v_{x}$ along the $x$-axis. Equation (1) can therefore be written as

$$
\rho \frac{\partial v_{x}}{\partial t}=-\frac{\Delta p}{\Delta L}+\eta\left(\frac{\partial^{2} v_{x}}{\partial y^{2}}+\frac{\partial^{2} v_{x}}{\partial z^{2}}\right)
$$

where we also note that the only driving pressure drop is the drop along the $\mathrm{x}$-axis of the channel given by $\frac{\Delta p}{\Delta L}$. 


\subsection{Numerical Scheme for the Second-Order Partial Differential Equations} given by

In [6] we derived numerical schemes for second-order partial differentials from the Taylor series

$$
\frac{d^{2} f}{d x^{2}}\left(x_{0}\right)=\frac{f\left(x_{0}+\Delta x\right)+f\left(x_{0}-\Delta x\right)-2 f\left(x_{0}\right)}{(\Delta x)^{2}}
$$

which allowed us to rewrite the right-hand side of Equation (2) to

$$
\begin{gathered}
\rho \frac{\partial v_{x}}{\partial t}=-\frac{\Delta p}{\Delta L}+ \\
\eta\left(\frac{v_{x}\left(y_{0}+\Delta y, z_{0}\right)+v_{x}\left(y_{0}-\Delta y, z_{0}\right)-2 v_{x}\left(y_{0}, z_{0}\right)}{(\Delta y)^{2}}+\frac{v_{x}\left(y_{0}, z_{0}+\Delta z\right)+v_{x}\left(y_{0}, z_{0}-\Delta z\right)-2 v_{x}\left(y_{0}, z_{0}\right)}{(\Delta z)^{2}}\right)
\end{gathered}
$$

which we can further simplify by using a common step width in space $h_{y z}=\Delta y=\Delta z$ to yield

$$
\eta\left(\frac{v_{x}\left(y_{0}+h_{y z}, z_{0}\right)+v_{x}\left(y_{0}-h_{y z}, z_{0}\right)+v_{x}\left(y_{0}, z_{0}+h_{y z}\right)+v_{x}\left(y_{0}, z_{0}-h_{y z}\right)-4 v_{x}\left(y_{0}, z_{0}\right)}{h_{y z}^{2}}\right)
$$

This scheme uses a so-called finite difference scheme to approximate the second order partial differential by considering the changes in the function over a finite difference in the independent variables $y$ and $z$. This scheme is a second-order scheme and thus numerically very stable.

Introducing the general notation $F^{(t, y, z)}$ for the value of $v_{x}$ at the position $\left(y_{0}, z_{0}\right)$ at time point $t$, $F^{(t, y+1, z)}$ for the value of $v_{x}$ at the position $\left(y_{0}+h_{y z}, z_{0}\right)$ at time point $t, F^{(t, y-1, z)}$ for the value of $v_{x}$ at the position $\left(y_{0}-h_{y z}, z_{0}\right)$ at time point $t, F^{(t, y, z+1)}$ for the value of $v_{x}$ at the position $\left(y_{0}, z_{0}+h_{y z}\right)$ at time point $t$ and $F^{(t, y, z-1)}$ for the value of $v_{x}$ at the position $\left(y_{0}, z_{0}-h_{y z}\right)$ at time point $t$ we can rewrite Equation (5) to

$$
\begin{aligned}
\frac{\partial v_{x}}{\partial t} & =-\frac{\Delta p}{\Delta L}+\eta\left(\frac{F^{(t, y+1, z)}+F^{(t, y-1, z)}+F^{(t, y, z+1)}+F^{(t, y, z-1)}-4 F^{(t, y, z)}}{h_{y z}^{2}}\right) \\
\frac{\partial v_{x}}{\partial t} & =-\frac{1}{\rho} \frac{\Delta p}{\Delta L}+\frac{\eta}{\rho}\left(\frac{F^{(t, y+1, z)}+F^{(t, y-1, z)}+F^{(t, y, z+1)}+F^{(t, y, z-1)}-4 F^{(t, y, z)}}{h_{y z}^{2}}\right)
\end{aligned}
$$

\subsection{Numerical Scheme for the First-Order Partial Differential with Respect to Time}

The second partial differential required for setting up the numerical scheme is the first-order partial differential with respect to time. For this we expand the Fourier series in the positive direction given by

$$
f\left(x_{0}+\Delta x\right)=\left.\sum_{n=0}^{n_{\max }} \frac{1}{n !} \frac{d^{n} f}{d x^{n}}\right|_{x_{0}} \Delta x^{n}+O_{n_{\max }+1}
$$

to $n_{\max }=1$, where $n_{\max }$ is the "expansion order" and $O_{n_{\max }}$ is the error function of order $n_{\max }$. We subsequently obtain

$$
\begin{gathered}
f\left(x_{0}+\Delta x\right)=f\left(x_{0}\right)+\frac{d f}{d x} \Delta x+O_{2} \\
\frac{d f}{d x}=\frac{f\left(x_{0}+\Delta x\right)-f\left(x_{0}\right)}{\Delta x}
\end{gathered}
$$

which is a first-order approximation for the first derivative in time (a so-called forward Euler scheme). Combining Equations (8) and (6) results in the numerical scheme

$$
\frac{F^{(t+1, y, z)}-F^{(t, y, z)}}{h_{t}}=-\frac{1}{\rho} \frac{\Delta p}{\Delta L}+\frac{\eta}{\rho}\left(\frac{F^{(t, y+1, z)}+F^{(t, y-1, z)}+F^{(t, y, z+1)}+F^{(t, y, z-1)}-4 F^{(t, y, z)}}{h_{y z}^{2}}\right)
$$




$$
\begin{gathered}
F^{(t+1, y, z)}=F^{(t, y, z)}-\frac{h_{t}}{\rho} \frac{\Delta p}{\Delta L}+\frac{h_{t} \cdot \eta}{\rho}\left(\frac{F^{(t, y+1, z)}+F^{(t, y-1, z)}+F^{(t, y, z+1)}+F^{(t, y, z-1)}-4 F^{(t, y, z)}}{h_{y z}^{2}}\right) \\
F^{(t+1, y, z)}=F^{(t, y, z)}\left(1-4 \frac{h_{t} \cdot \eta}{\rho h_{y z}^{2}}\right)+\frac{h_{t} \cdot \eta}{\rho}\left(\frac{F^{(t, y+1, z)}+F^{(t, y-1, z)}+F^{(t, y, z+1)}+F^{(t, y, z-1)}}{h_{y z}^{2}}\right)-\frac{h_{t}}{\rho} \frac{\Delta p}{\Delta L} \\
F^{(t+1, y, z)}=F^{(t, y, z)}(1-4 \Omega)+\Omega\left(F^{(t, y+1, z)}+F^{(t, y-1, z)}+F^{(t, y, z+1)}+F^{(t, y, z-1)}\right)-\Gamma
\end{gathered}
$$

with $\Omega=\frac{h_{t} \Delta \eta}{\rho h_{y z}^{2}}$ and $\Gamma=\frac{h_{t}}{\rho} \frac{\Delta p}{\Delta L}, h_{t}$ being the step width in time. Equation (9) is a second-order scheme with respect to space and a first-order scheme with respect to time. It allows stepping forward in time from a known value $F^{(t, y, z)}$ at the location $(x, y)$ at time $t$ to the unknown value $F^{(t+1, y, z)}$ at the location $(x, y)$ at time $t+1$. Compared to the schemes used in [6] this scheme is not iterative as the solution at a given time point $t$ does not have to fulfill the Navier-Stokes equation but only Equation (8). One point of note is the fact that the numerical scheme in time is only first-order. In order to not risk numerical instability the step width $h_{t}$ in time must be chosen sufficiently small. In general, first-order approximations are numerically significantly less stable than higher-order implementations but they are computationally very cost-effective and thus very simple to implement. The numerical stability of the overall scheme hinges mostly on the step width in time.

\subsection{Correcting Units}

Before implementing the numerical scheme we must correct for the units. We assume $h_{x y}$ to be given in $\mu \mathrm{m}, \frac{\Delta p}{\Delta L}$ to be given in mbar $/ \mathrm{mm}, \rho$ to be given in $\mathrm{g} / \mathrm{cm}^{3}, \eta$ to be given in $\mathrm{mPa} \cdot \mathrm{s}$ and $h_{t}$ to be given in $\mu$ s. The unit of the dependent variable $v_{x}$ is $\mathrm{mm} / \mathrm{s}$ and the independent variables $y$ and $z$ are given in $\mu \mathrm{m}$. In order to correct for the units, $\Gamma$ has a prefactor of 0.1 , whereas $\Omega$ has a prefactor of 1 .

\section{Implementation in Microsoft Excel}

\subsection{Layout of the Spreadsheet}

The scheme given by Equation (9) was implemented in Microsoft Excel in a spreadsheet, which can be downloaded from the supporting material (file "TimedependentMicrofluidicFlows.xlsx"). It is shown in Figure 1. The numerical domain was chosen as a $40 \times 40$ cell grid panel with no-slip boundary conditions. As demonstrated in an earlier contribution, different boundary conditions can be implemented such as, e.g., flip or Neumann-type boundary conditions [7]. The values in the cells represent the velocity of the flow at the given position in the domain. The sheet consists of three panels:

- left panel-initial conditions: these are the values of the flow in the channel at the beginning of the calculation; for a first demonstration, we assume the flow to be non-moving, i.e., all values are 0

- center panel—velocity profile at time point $t$ : this is the velocity profile in the channel at the current timepoint, i.e., $F^{(t, y, z)}$; the scheme is assumed to step from this point to $F^{(t+1, y, z)}$

- $\quad$ right panel一velocity profile at time point $t+h_{t}$ : this is the velocity profile calculated by stepping from timepoint $t$ via the numerical scheme of Equation (9)

The panels are color-coded to reflect areas of higher velocity in red and areas of lower velocity in green. Next to the right-most panel, the color scale for the velocity profile for the right-most panel, i.e., $F^{(t+1, y, z)}$ is displayed. Below the color scale is the section for the variables. These values can be changed to modify, i.e., the type of fluid or the properties of the numerical scheme. The numerical scheme is corrected for the following units:

- $\quad$ independent variables $y$ and $z: \mu \mathrm{m}$

- $\quad$ pressure drop $\frac{\Delta p}{\Delta L}: \mathrm{mbar} / \mathrm{mm}$

- $\quad$ step width in space $h_{y z}: \mu \mathrm{m}$

- $\quad$ step width in time $h_{t}: \mu \mathrm{s}$ 
- density of the fluid: $\mathrm{g} / \mathrm{cm}^{3}$

- $\quad$ viscosity of the fluid: $\mathrm{mPa} \cdot \mathrm{s}$

Changing the value of the step width in space effectively increases the lateral dimension of the channel. Changing the value of the step width in time increases the speed of the calculation by assuming larger steps in the forward Euler scheme. However, as discussed, increasing this value may lead to the numerical scheme becoming unstable. This can be observed by the values of the velocity increasing continually until they overflow. Below the adjustable variables are the two variables used as an abbreviation in Equation (9), i.e., $\Omega$ and $\Gamma$ which are updated dynamically.

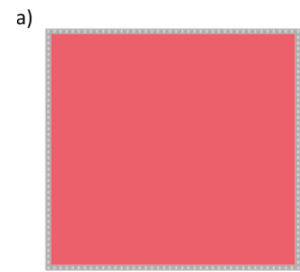

Initial conditions

b)

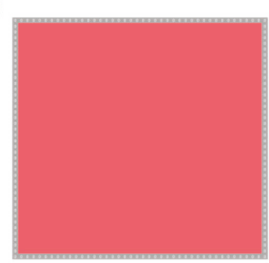

Initial conditions

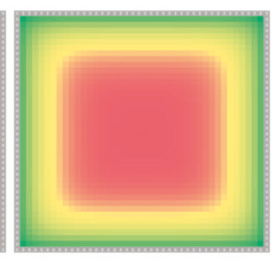

Timepoint t Iteration 123

Time [us] 123 Reset

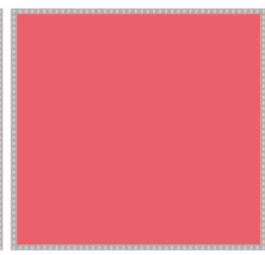

Timepoint $t$ Iteration 0

Time $[\mu \mathrm{s}] 0$ Reset yes

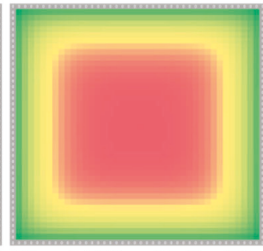

Timepoint t+ht Iteration 123

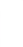

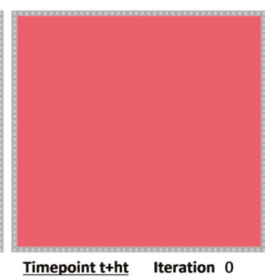

Timepoint $t+h t$ Iteration 0

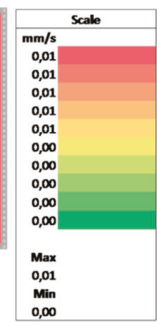

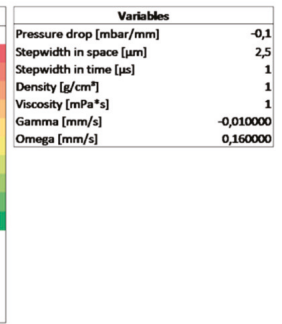

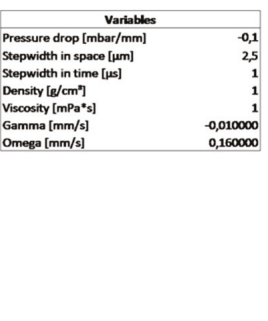

Figure 1. View of the Microsoft Excel spreadsheets with the three panels: initial conditions (left), current time point (center) and next time point (right). (a) The evolution of the velocity profile can be observed by pressing the F9 key. The right panel implements Equation (9) and steps forward in time. The values are copied back to the center panel thus performing one iteration. (b) By adding any value into the "Reset" field, the scheme is reset, the iteration counter is cleared and the values of the initial condition (left panel) is copied into the center panel thus setting the velocity profile for time point $t=0$.

\subsection{Iteration}

As discussed, the numerical scheme of Equation (9) does not require iteration within one time point. This is in contrast to the schemes implemented in [6], which require the steady-state version of the Poiseuille Equation (2) to be fulfilled for each position within the domain. Here, the scheme is required to perform one step in time but not to iterate further.

However, due to the nature of the scheme, we require circular references in the spreadsheet, which means we have to allow iteration. In Microsoft Excel, select "File $\rightarrow$ Options $\rightarrow$ Formulas $\rightarrow$ Calculation options" and check "Enable iterative calculation". Set "Maximum Iterations" to 1 . This ensures that the scheme will only perform one single iteration.

\subsection{Implementation of the Numerical Scheme}

The scheme is implemented in the spreadsheet by taking the initial value from the left panel and copying the values into the center panel at the beginning of the calculation. The scheme then uses these values to calculate $F^{(t+1, y, z)}$ in the right panel from the values $F^{(t, y, z)}$ of the center panel. For the next step in time, the value of the right panel is copied back into the respective cell of the center 
panel. This is the single iteration which Microsoft Excel will perform-the value will not be updated further. The formulae of the cells in the right panel implement the numerical scheme. They use the value $F^{(t, y, z)}$ as well as $F^{(t, y+1, z)}, F^{(t, y-1, z)}, F^{(t, y, z+1)}$ and $F^{(t, y, z-1)}$ from the center panel, as well as the values $\Omega$ and $\Gamma$. For each step in time, the scheme will update the values in the right panel from the values of the center panel and write these values back to the center grip. By pressing F9 or performing any recorded input in Microsoft Excel an additional step in time will be performed. Below the center and the right panel are iteration counters that increment any time an input key or F9 (which triggers a spreadsheet recalculation) is recorded. By keeping F9 pressed, the evolution of the flow profile in time can be observed. Each step correlates to a step in time of $h_{t}$. An additional field is added to calculate the total number of microseconds passed since the beginning of the calculation.

\subsection{Resetting the Calculation and Implementing the Boundary Conditions}

Upon close inspection, the cells in the center panel do not simply copy the values from the right panel. They are linked by a conditional expression. If a certain field below the center panel (the field labeled "Reset") is empty, the value from the right panel is copied. If the "Reset" field is not empty, the value from the left panel is copied. This effectively resets the calculation and also clears the iteration counters, which are implemented with a similar conditional copy operation. Writing any letter, value or number into the "Reset" field will thus reset the calculation and copy the initial conditions into the center panel corresponding to the velocity profile at $t=0$.

\section{Analytical Solution for Initiating Two-Dimensional Flow in Rectangular Channel Cross-Sections}

\subsection{Derivation}

In order to verify the correctness of the numerical results obtained from the implemented solver in Microsoft Excel, we chose the case of initiating two-dimensional flow in a rectangular channel cross-section with the no-slip boundary condition as an example. In this scenario, the flow in the channel is originally at rest. At $t=0$, a driving pressure gradient is applied along the length of the channel thus initiating the flow whereby the characteristic velocity profile in a rectangular channel cross-section Poiseuille flow will form. The dynamics of the evolution of this flow profile will be studied using the derived analytical solution which yields the exact results. These will then be compared to the numerical results obtained from the spreadsheet.

The relevant partial differential equation for this case is Equation (2) which is rewritten to

$$
\frac{\rho}{\eta} \frac{\partial v_{x}}{\partial t}-\left(\frac{\partial^{2} v_{x}}{\partial y^{2}}+\frac{\partial^{2} v_{x}}{\partial z^{2}}\right)=-\frac{\Delta p}{\eta \Delta L}
$$

We assume the solution to consist of a steady-state component which is time-independent and a transient solution which is time-dependent. The latter will be dominating during the initiation of the flow and decline as the flow achieves steady-state. Thus, the general solution will be

$$
v_{x}(t, y, z)=v_{x, \text { steady-state }}(y, z)+v_{x, \text { transient }}(t, y, z)
$$

If inserted into Equation (10) we obtain

$$
\begin{aligned}
& \frac{\rho}{\eta}\left(\frac{v_{x, \text { transient }}}{\partial t}\right)-\left(\frac{\partial^{2} v_{x, \text { steady-state }}}{\partial y^{2}}+\frac{\partial^{2} v_{x, \text { steady-state }}}{\partial z^{2}}+\frac{\partial^{2} v_{x, \text { transient }}}{\partial y^{2}}+\frac{\partial^{2} v_{x, \text { transient }}}{\partial z^{2}}\right) \\
&=-\frac{\Delta p}{\eta \Delta L}
\end{aligned}
$$

Please note that $\frac{\partial v_{x, \text { steady-state }}}{\partial t}=0$. The steady-state solution $v_{x, \text { steady-state }}$ for this flow case is known and was derived in a similar case in Richter et al. [7] as Equation 13' using an eigenvalue expansion 
for mixed boundary cases. The solution for no-slip boundary conditions is derived elsewhere ([8], Equation 16.62)

$$
\begin{gathered}
v_{x, \text { steady-state }}(y, z)=-\frac{16}{\eta \cdot \pi^{2}} \frac{\Delta p}{\Delta L} \sum_{n=0}^{\infty} \sum_{m=0}^{\infty} \sin \left((2 n+1) \pi \frac{y}{W}\right) \sin \left((2 m+1) \pi \frac{z}{H}\right) \\
\left((2 n+1)(2 m+1)\left(\left(\frac{(2 n+1) \pi}{W}\right)^{2}+\left(\frac{(2 m+1) \pi}{H}\right)^{2}\right)\right)^{-1}
\end{gathered}
$$

The second-order partial differentials required for Equation (12) are given by

$$
\begin{aligned}
\frac{\partial^{2} v_{x, \text { steady-state }}}{\partial y^{2}}= & \frac{16}{\eta \cdot \pi^{2}} \frac{\Delta p}{\Delta L} \sum_{n=0}^{\infty} \sum_{m=0}^{\infty}\left(\frac{(2 n+1) \pi}{W}\right)^{2} \sin \left((2 n+1) \pi \frac{y}{W}\right) \sin \left((2 m+1) \pi \frac{z}{H}\right) \\
& \left((2 n+1)(2 m+1)\left(\left(\frac{(2 n+1) \pi}{W}\right)^{2}+\left(\frac{(2 m+1) \pi}{H}\right)^{2}\right)\right)^{-1} \\
\frac{\partial^{2} v_{x, \text { steady-state }}}{\partial z^{2}}= & \frac{16}{\eta \cdot \pi^{2}} \frac{\Delta p}{\Delta L} \sum_{n=0}^{\infty} \sum_{m=0}^{\infty}\left(\frac{(2 m+1) \pi}{H}\right)^{2} \sin \left((2 n+1) \pi \frac{y}{W}\right) \sin \left((2 m+1) \pi \frac{z}{H}\right) \\
& \left((2 n+1)(2 m+1)\left(\left(\frac{(2 n+1) \pi}{W}\right)^{2}+\left(\frac{(2 m+1) \pi}{H}\right)^{2}\right)\right)^{-1} \\
\sum_{n=0}^{\infty} \sum_{m=0}^{\infty} \sin \left((2 n+1) \pi \frac{y}{W}\right) \sin \left((2 m+1) \pi \frac{z}{H}\right)((2 n+1)(2 m+1))^{-1} &
\end{aligned}
$$

Equation (16) is the representation of a constant by a two-dimensional Fourier series. Details on this can be found elsewhere ([8], Equation 4.44). We can thus simplify Equation (16) to

$$
\frac{\partial^{2} v_{x, \text { steady-state }}}{\partial y^{2}}+\frac{\partial^{2} v_{x, \text { steady-state }}}{\partial z^{2}}=\frac{\Delta p}{\eta \Delta L}
$$

Using Equation (17), we can rewrite Equation (12) to

$$
\frac{\rho}{\eta} \frac{v_{x, \text { transient }}}{\partial t}-\frac{\partial^{2} v_{x, \text { transient }}}{\partial y^{2}}-\frac{\partial^{2} v_{x, \text { transient }}}{\partial z^{2}}=0
$$

Equation (18) is a homogeneous partial differential equation which can be solved by a separation of variables approach using

$$
v_{x, \text { transient }}=T(t) Y(y) Z(z)
$$

Inserting Equation (19) into Equation (18) yields

$$
\frac{\rho}{\eta} \frac{1}{T} \frac{\mathrm{dT}}{d t}-\frac{1}{Y} \frac{d^{2} \mathrm{Y}}{d y^{2}}-\frac{1}{Z} \frac{d^{2} \mathrm{Z}}{d z^{2}}=0
$$

from which we derive the two second-order ordinary differential equations

$$
\begin{aligned}
& \frac{1}{Y} \frac{d^{2} Y}{d y^{2}}=-\lambda_{Y}^{2} \\
& \frac{1}{Z} \frac{d^{2} Z}{d z^{2}}=-\lambda_{Z}^{2}
\end{aligned}
$$

and one first-order differential equation

$$
\frac{\rho}{\eta} \frac{1}{T} \frac{\mathrm{dT}}{d t}=-\left(\lambda_{Y}^{2}+\lambda_{Z}^{2}\right)
$$


The solution to Equation (23) is straight-forward and given by integration as

$$
T(t)=C_{0} e^{-\left(\lambda_{Y}^{2}+\lambda_{Z}^{2}\right) \frac{\eta}{\rho} t}
$$

The solutions to Equations (21) and (22) are given by the eigenfunctions and derived in a similar fashion as shown in [7] to be

$$
\begin{aligned}
& Y(y)=\sum_{n=0}^{\infty} C_{n} \sin \left(n \pi \frac{y}{W}\right), \lambda_{Y}=\frac{n \pi}{W} \\
& Z(z)=\sum_{m=0}^{\infty} C_{m} \sin \left(m \pi \frac{z}{H}\right), \lambda_{Z}=\frac{m \pi}{H}
\end{aligned}
$$

Inserting Equations (24), (25) and (26) into Equation (19) yields the transient solution as

$$
v_{x, \text { transient }}(t, y, z)=e^{-\left(\lambda_{Y}^{2}+\lambda_{Z}^{2}\right) \frac{\eta}{\rho} t} \sum_{n=0}^{\infty} \sum_{m=0}^{\infty} C_{n m} \sin \left(n \pi \frac{y}{W}\right) \sin \left(m \pi \frac{z}{H}\right)
$$

We still lack the constant $C_{n m}$, which we can derive from the initial condition of the accelerating flow which requires the flow to be constant, i.e., $v_{x}=0$. This is the case for $v_{x, \text { transient }}(t=0, y, z)=$ $-v_{x, \text { steady-state }}(0, y, z)$. In this case we find

$$
\begin{aligned}
\sum_{n=0}^{\infty} \sum_{m=0}^{\infty} C_{n m} & \sin \left((2 n+1) \pi \frac{y}{W}\right) \sin \left((2 m+1) \pi \frac{z}{H}\right) \\
& =\frac{16}{\eta \cdot \pi^{2}} \frac{\Delta p}{\Delta L} \sum_{n=0}^{\infty} \sum_{m=0}^{\infty} \sin \left((2 n+1) \pi \frac{y}{W}\right) \sin \left((2 m+1) \pi \frac{z}{H}\right) \\
& \left((2 n+1)(2 m+1)\left(\left(\frac{(2 n+1) \pi}{W}\right)^{2}+\left(\frac{(2 m+1) \pi}{H}\right)^{2}\right)\right)^{-1}
\end{aligned}
$$

from which we find

$$
C_{n m}=\frac{16}{\eta \cdot \pi^{2}} \frac{\Delta p}{\Delta L}\left((2 n+1)(2 m+1)\left(\left(\frac{(2 n+1) \pi}{W}\right)^{2}+\left(\frac{(2 m+1) \pi}{H}\right)^{2}\right)\right)^{-1}
$$

Assembling Equation (11) from Equations (13), (27) and (29) we find

$$
\begin{aligned}
& v_{x}(t, y, z)=-\frac{16}{\eta \cdot \pi^{2}} \frac{\Delta p}{\Delta L} \sum_{n=0}^{\infty} \sum_{m=0}^{\infty} \frac{\left(1-e^{-\left(\left(\frac{(2 n+1) \pi}{W}\right)^{2}+\left(\frac{(2 m+1) \pi}{H}\right)^{2}\right) \frac{\eta}{\rho} t}\right)}{\sin \left((2 n+1) \pi \frac{y}{W}\right) \sin \left((2 m+1) \pi \frac{z}{H}\right)} \\
&\left((2 n+1)(2 m+1)\left(\left(\frac{(2 n+1) \pi}{W}\right)^{2}+\left(\frac{(2 m+1) \pi}{H}\right)^{2}\right)\right)^{-1}
\end{aligned}
$$

Visualization

In the following, Equation (30) is visualized using a microfluidic channel with $100 \mu \mathrm{m}$ width and $100 \mu \mathrm{m}$ height and choosing water $\left(\eta=1 \mathrm{mPa} \cdot \mathrm{s}, \rho=1 \mathrm{~g} / \mathrm{cm}^{3}\right)$ as the fluid. The Fourier series in Equation (30) is expanded to $n_{\max }=m_{\max }=10$. Figure 2 shows the calculated profiles for $10 \mu \mathrm{s}$, $100 \mu \mathrm{s}$ and $1000 \mu \mathrm{s}$. As can be seen the fluid is originally at rest. Over time, the characteristic profile of the Poiseuille flow in a rectangular channel cross-section evolves. By inspection of Equations (27) and (30), this behavior is expected. As the solution consists of a transient and a steady-state component, the first of which decays exponentially over time, the steady-state solution is effectively modulated by the exponential decay of the time-dependent contribution of the transient solution. 


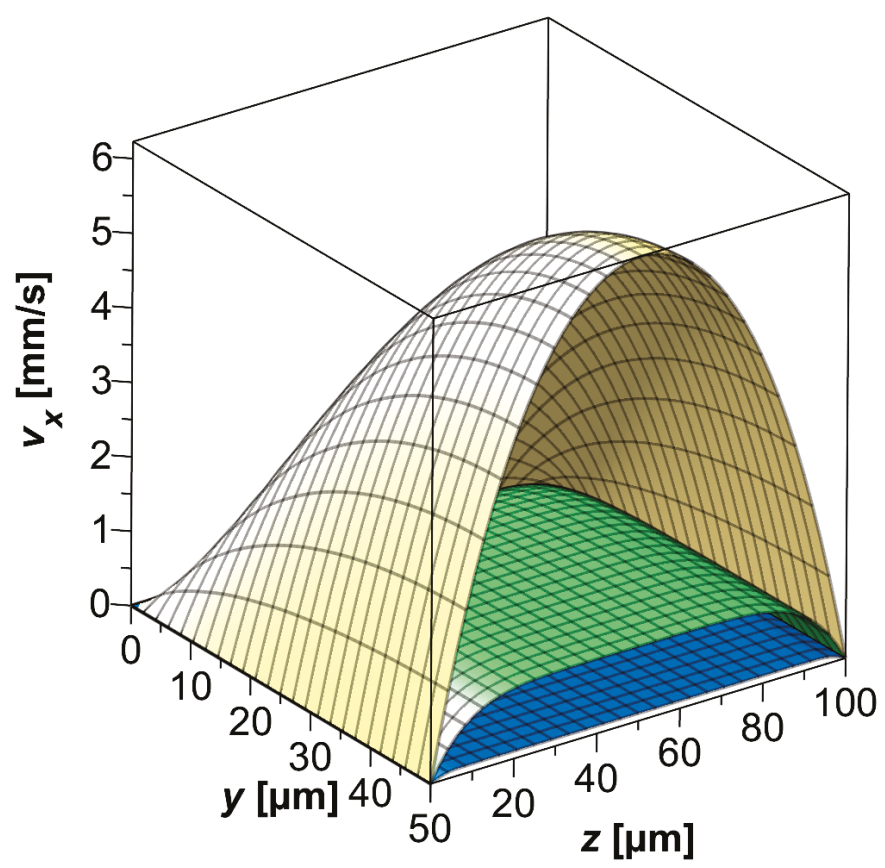

Figure 2. Velocity profile calculated from the analytical solution of Equation (30) for water as fluid and a channel with $100 \mu \mathrm{m}$ width and $100 \mu \mathrm{m}$ height. The Fourier series is expanded to $n_{\max }=m_{\max }=10$. The expansion order of the Fourier series was 10 both along $y$ and $z$. Velocity profiles for different points in time are plotted: $10 \mu$ s (dark blue), $100 \mu$ s (medium green) and $1000 \mu$ s (light yellow).

\subsection{Application of the Derived Spreadsheet}

\subsubsection{Initiating Two-Dimensional Flow in Rectangular Channel Cross-Sections}

Given the analytical solution Equation (30) we now proceed to solving the same case using the numerical solver implemented in the spreadsheet. The step width in space was chosen as $h_{y z}=2.5 \mu \mathrm{m}$ and the step width in time as $h_{t}=1 \mu \mathrm{s}$. Again, water as the fluid was assumed and thus $\eta=1 \mathrm{mPa} \cdot \mathrm{s}$, $\rho=1 \mathrm{~g} / \mathrm{cm}^{3}$ were set in the "Variables" section of the spreadsheet. For direct comparison with Figure 2, the spreadsheet was iterated 100 times to yield the velocity profile at $t=100 \mu \mathrm{s}$ and $t=1000 \mu \mathrm{s}$. The resulting velocity profile was then compared with the analytical Page: 10 solution given by Equation (30). Figure 3 shows the relative error of the numerical output compared to the analytical solution. The maximum error in the whole computational domain is below $3 \%$ relative error in both cases. The errors are strongest in regions of steep gradients in the velocity profiles, i.e., at the edges. In order to decrease the error further, the computational domain can be increased by increasing the number of cells. However, even on this rather coarse and compact domain, the errors fall within acceptable limits. 
a)

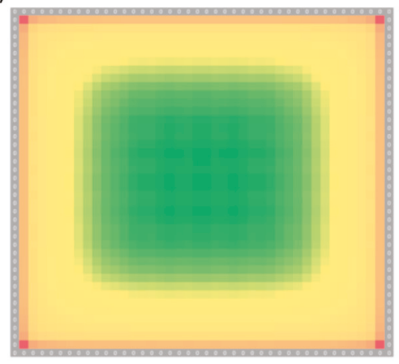

b)

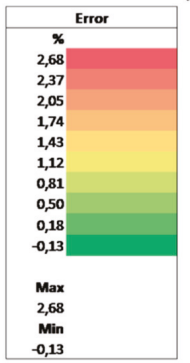

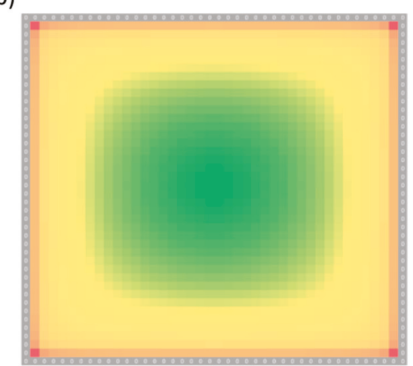

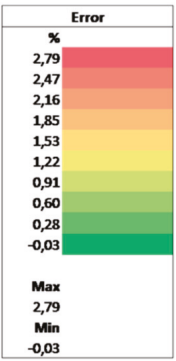

Figure 3. Comparison of the results obtained from the numerical scheme implemented in Microsoft Excel against the analytical solution of Equation (30) for time point $100 \mu \mathrm{s}$ (a) and $1000 \mu \mathrm{s}$ (b). The graphs show the relative error. Both cases show very good agreements with maximum errors below $3 \%$.

\subsubsection{Complex Flow Cases: Different Channel Cross-Sections}

As shown, the analytical solution can be derived for the (rather simplistic) case of rectangular channel cross-sections. However, the availability of these solutions is limited if the channel geometries become more complex. However, these cross-sections are straight-forward to implement in the spreadsheet. Figure 4a shows the example of a rectangular channel with two fins constricting the flow.

a)

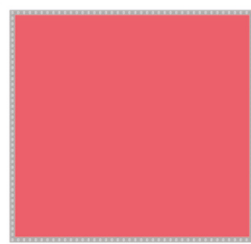

Initial conditions

"

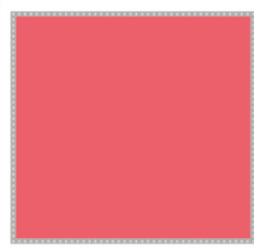

Initial conditions
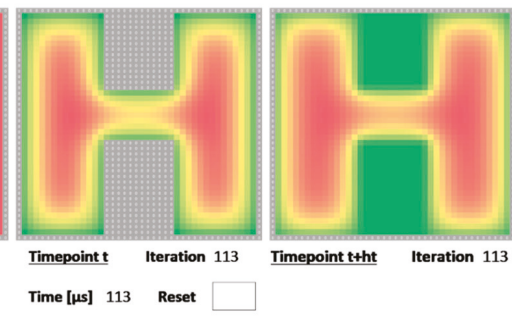

Timepoint t+ht Iteration 113

Time [us] 113 Res

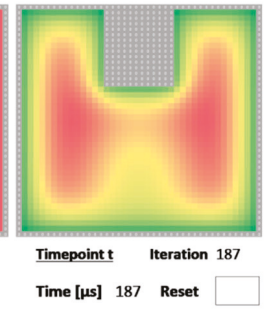

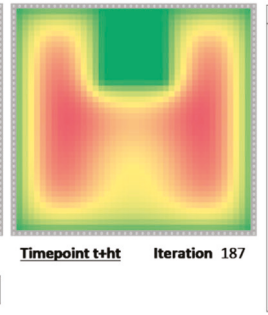
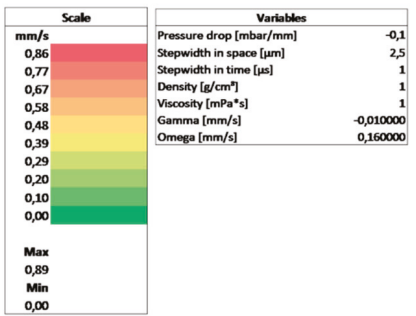

Figure 4. Complex flow cases involving intricate domains such as a double-constricted channel cross-section. (a) These are implemented by adding additional boundary values, i.e., by copying the grey cell values which have static values. The image shows the flow after around 100 iterations. (b) By simple overwriting portions of the boundary values with domain values, i.e., reimplementing the conditional copy operation of the center values, one of the obstacles is removed at a given point in time. The scheme can then be iterated to see how the flow adapts to the changes in boundary conditions.

These can be implemented by copying additional boundary values into the center panel. By overwriting the formulae in these cells, the numerical scheme cannot iterate these values. However, they influence neighboring cells and thus show up as regions with no flow in the iterated panel on the right. Again, starting with a static flow, the scheme can be used to observe the development of the velocity profile over time. Figure 4 a shows the profile at iteration $\# 113$, i.e., at $t=113 \mu$ s. Boundary 
conditions can be changed dynamically. As an example, the lower constriction in the channel of Figure $4 \mathrm{a}$ was removed at $t=113 \mu$ s. Figure $4 \mathrm{~b}$ shows the adjusting flow 74 iterations after the object was removed. As can be seen, the flow re-expands into the regions blocked previously. Removing a boundary can be accomplished by simply recopying the formulae from the domain into the center panel thus reactivating the iteration.

\subsubsection{Boundary Conditions and Initial Conditions}

As discussed, boundary conditions with fixed values (Dirichlet-type) can be implemented by overwriting cells in the center panel with fixed values. No-slip boundary conditions are implemented by setting this value to 0 . Surfaces with fixed velocity as in the case of, e.g., Couette flow, can be implemented by setting the velocity of these boundary cells to a fixed non-zero value. As demonstrated in [7], it is possible to also implement Neumann-type boundary conditions by simply setting the cell values of the boundary to the value of the adjacent cell within the computational domain thus effectively generating a velocity gradient of 0 as required for, e.g., slip boundary conditions. Obviously, the gradient can also be set to a fixed value by simply setting the value of the boundary cell to the value of the neighboring cell within the computational domain plus a fixed offset value.

It is also possible to use non-zero initial values for computation. Until now, we assumed the flow to be static at the beginning of the experiment and to increase due to the application of the flow as a consequence of the application of a pressure gradient. This spreadsheet analyses the declining velocity profile of an initially moving fluid as a consequence of the removal of the driving pressure. The initial values are copied from a spreadsheet that iterated until the velocity profile was fully developed. The spreadsheet of Figure 5 sets the driving pressure gradient to 0 . As you can see, after 1000 iterations the velocity profile has smeared slightly but the overall distribution is still similar to the case of the fully-developed profile. This is to be expected from the analytical solution to Equation (27). For a decelerating flow, the steady-state fully-developed flow profile would be modulated by an exponential term, thus generating an exponential decay. As a consequence, the flow profiles would simply be scaled but the shape of the profile would remain largely intact. As can be seen from Figure 5, after 1000 iterations, the maximum velocity in the channel has decreased to a value of below $0.5 \mathrm{~mm} / \mathrm{s}$.

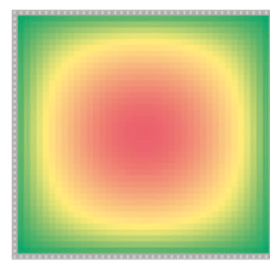

Initial conditions

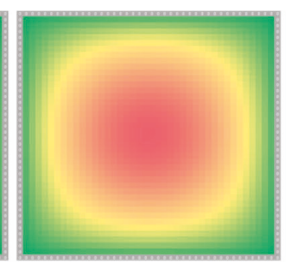

Timepoint t Iteration 1688

Time [ $\mu \mathrm{s}] 1.688$ Reset

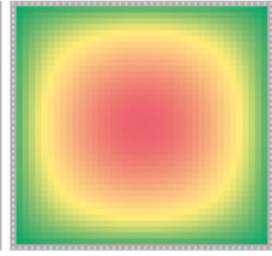

Timepoint t+ht Iteration 1688

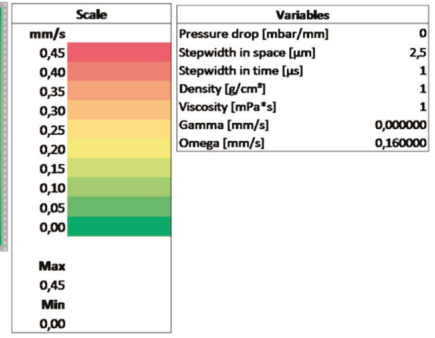

Figure 5. Example of a flow case using boundary conditions. The demonstrated case is a decelerating flow which was initially fully-defined as indicated by the initial conditions. At $t=0$ the driving pressure gradient is removed and the flow begins to stagnate. After more than 1500 iterations, the velocity profile is smeared and the velocity profile drops to maximum values below $0.5 \mathrm{~mm} / \mathrm{s}$.

\section{Conclusions}

Solutions to the simplified Navier-Stokes equation in pressure-driven microfluidics, i.e., Poiseuille flows, are difficult to derive analytically if the channel cross-sections, the boundary conditions or the initial values do not represent trivial cases. Including time-dependency further complicates the derivation of the analytical solutions. These cases are usually addressed using numerical solvers and dedicated software packages. However, as we have shown, a numerical solver suitable for solving time-dependent microfluidic flow cases in arbitrary channel cross-sections can be conveniently 
implemented using spreadsheet analysis tools such as, e.g., Microsoft Excel. By making use of a controlled iterative calculation, the solver can be stepped in time by manual input thus allowing a precise study of the evolving velocity profiles over time at discrete time points. No additional software is required for obtaining results that almost exactly correspond to the precise analytical solution. We demonstrated this using the case of time-dependent initiating flow in a rectangular channel cross-section. The spreadsheet developed can be used to implement almost any type of boundary condition or initial condition, as well as channel cross-section as required. We believe that providing researchers with intuitive and widely accessible numerical tools will significantly increase the understanding and the correct derivation of the fluid mechanics in microfluidics with implications for applications in liquid delivery, reaction synthesis and analytical applications [9-11]. Spreadsheet software packages such as, e.g., Microsoft Excel are widely available and most scientists are used to working with these tools in routine lab work. Using these tools effectively to provide such detailed insight into fluid mechanics will significantly widen the application range and provide more detailed understanding of phenomena which are generally only accessible with specialized software packages.

Supplementary Materials: The following are available online at http://www.mdpi.com/2079-6374/9/2/67/s1, Microsoft Excel spreadsheet "TimedependentMicrofluidicFlows.xlsx".

Author Contributions: Conceptualization, P.R., F.K. and B.R.; methodology, P.R., D.H. and B.E.R.; software, B.R.; writing-P.R. and D.H.; writing-review and editing, B.R.; visualization, F.K. and B.R.; supervision, D.H. and B.R.; project administration, B.R.; funding acquisition, B.R.

Funding: This work was funded by the Bundesministerium für Bildung und Forschung (BMBF), funding code 03X5527 "Fluoropor" and by the Baden-Wuerttemberg Stiftung, program "biofunctional materials and surfaces".

Conflicts of Interest: The authors declare no conflict of interest.

\section{References}

1. Vijayendran, R.A.; Motsegood, K.M.; Beebe, D.J.; Leckband, D.E. Evaluation of a three-dimensional micromixer in a surface-based biosensor. Langmuir 2003, 19, 1824-1828. [CrossRef]

2. Baronas, R.; Ivanauskas, F.; Kulys, J. The effect of diffusion limitations on the response of amperometric biosensors with substrate cyclic conversion. J. Math. Chem. 2004, 35, 199-213. [CrossRef]

3. Li, Y.; Van Roy, W.; Lagae, L.; Vereecken, P.M. Analysis of fully on-chip microfluidic electrochemical systems under laminar flow. Electrochim. Acta 2017, 231, 200-208. [CrossRef]

4. Squires, T.M.; Quake, S.R. Microfluidics: Fluid physics at the nanoliter scale. Rev. Mod. Phys. 2005, 77, 977-1026. [CrossRef]

5. Koo, J.M.; Kleinstreuer, C. Liquid flow in microchannels: Experimental observations and computational analyses of microfluidics effects. J. Micromech. Microeng. 2003, 13, 568-579. [CrossRef]

6. Richter, C.; Kotz, F.; Giselbrecht, S.; Helmer, D.; Rapp, B.E. Numerics made easy: Solving the navier-stokes equation for arbitrary channel cross-sections using microsoft excel. Biomed. Microdevices 2016, 18, 52. [CrossRef] [PubMed]

7. Richter, C.; Kotz, F.; Keller, N.; Nargang, T.M.; Sachsenheimer, K.; Helmer, D.; Rapp, B.E. An analytical solution to neumann-type mixed boundary poiseuille microfluidic flow in rectangular channel cross-sections (slip/no-slip) including a numerical technique to derive it. J. Biomed. Sci. Eng. 2017, 10, 205-219. [CrossRef]

8. Rapp, B.E. Microfluidics: Modeling, Mechanics and Mathematics; William Andrew: Amsterdam, The Netherlands, 2016.

9. Elvira, K.S.; Solvas, X.C.I.; Wootton, R.C.R.; de Mello, A.J. The past, present and potential for microfluidic reactor technology in chemical synthesis. Nat. Chem. 2013, 5, 905-915. [CrossRef] [PubMed]

10. Whitesides, G.M. The origins and the future of microfluidics. Nature 2006, 442, 368-373. [CrossRef] [PubMed]

11. Ohno, K.; Tachikawa, K.; Manz, A. Microfluidics: Applications for analytical purposes in chemistry and biochemistry. Electrophoresis 2008, 29, 4443-4453. [CrossRef] [PubMed] 
Article

\title{
Integrated Microfluidic Devices Fabricated in Poly (Methyl Methacrylate) (PMMA) for On-site Therapeutic Drug Monitoring of Aminoglycosides in Whole Blood
}

\author{
Zaidon T. Al-aqbi ${ }^{1,2}$, Yiing C. Yap ${ }^{1}$, Feng $\mathrm{Li}^{1}$ and Michael C. Breadmore ${ }^{1, *}$ \\ 1 Australian Centre for Research on Separation Science (ACROSS), School of Natural Sciences-Chemistry, \\ University of Tasmania, Private Bag 75, Hobart, Tasmania 7001, Australia; zthal@utas.edu.au (Z.T.A.); \\ yiing.yap@utas.edu.au (Y.C.Y.); Feng.Li@utas.edu.au (F.L.) \\ 2 College of Agriculture, University of Misan, Al-amarah, Misan 62001, Iraq \\ * Correspondence: Michael.breadmore@utas.edu.au
}

Received: 21 November 2018; Accepted: 19 December 2018; Published: 30 January 2019

\begin{abstract}
On-site therapeutic drug monitoring (TDM) is important for providing a quick and accurate dosing to patients in order to improve efficacy and minimize toxicity. Aminoglycosides such as amikacin, gentamicin, and tobramycin are important antibiotics that have been commonly used to treat infections of chronic bacterial infections in the urinary tract, lung, and heart. However, these aminoglycosides can lead to vestibular and auditory dysfunction. Therefore, TDM of aminoglycosides is important due to their ototoxicity and nephrotoxicity. Here, we have developed a hot embossed poly (methyl methacrylate) (PMMA) microfluidic device featuring an electrokinetic size and mobility trap (SMT) to purify, concentrate, and separate the aminoglycoside antibiotic drugs amikacin, gentamicin, and tobramycin. These drugs were separated successfully from whole blood within $3 \mathrm{~min}$, with 30 -fold lower detection limits compared to a standard pinched injection. The limit of detections (LOD) were $3.75 \mu \mathrm{g} / \mathrm{mL}$ for gentamicin, $8.53 \mu \mathrm{g} / \mathrm{mL}$ for amikacin, and $6.00 \mu \mathrm{g} / \mathrm{mL}$ for tobramycin. These are sufficient to cover the therapeutic range for treating sepsis of $6-10 \mu \mathrm{g} / \mathrm{mL}$ gentamicin and tobramycin and $12-20 \mu \mathrm{g} / \mathrm{mL}$ of amikacin. The device is simple and could be mass produced via embossing or injection molding approaches.
\end{abstract}

Keywords: therapeutic drug monitoring (TDM); aminoglycosides; size and mobility traps (SMT)

\section{Introduction}

Personalized medicine requires healthcare customization with medical practices, treatments, decisions, or products specific for each patient. It is most commonly associated with diagnostic testing to detect the early onset for a change in health state. When applied to personalized therapy, it involves choosing medication based on the patient's genetic content to identify the correct drug for treatment [1] and therapeutic drug monitoring (TDM) to ensure the correct dosing period and optimum concentration to minimize toxicity and improve efficacy [2,3]. TDM requires the monitoring of blood/plasma concentrations and is important when there is a narrow therapeutic range. TDM has been proven to progress anticancer therapies, such as methotrexate [4], as well as in new targeted anticancer agents, such as nilotinib, imatinib, sunitinib, dasatinib, lapatinib, and sorafenib [5], and is critical for immunospuressants [6]. TDM also shows benefits for a wide array of drugs, particularly antibacterials, anticonvulsants, antidepressants, antiretrovirals, antipsychotics, and $\beta$-lactam antibiotics [7]. There are a range of methods which can be used to conduct TDM, but it is typically carried out in a laboratory by using liquid chromatography mass spectrometry and immunoassays [8]. 
For some applications of TDM, there is a desire to either be able to test at-home, such as for patients receiving treatment for chronic conditions, or for a rapid and quick response to enable quicker and more efficient treatment. The most well-known example of TDM is measuring blood glucose levels such that a diabetic can self-dose the correct amount of insulin. There are now many different blood glucose analyzers available on the market for rapid on-site testing; however, these are predominantly based on either an antibody or an immunoassay. For targets where there is insufficient specificity in these approaches, they are usually analysed with a high resolution separation, which is much harder to miniaturise than an immunoassay. This capability has been recently demonstrated through the introduction of a portable electrophoretic device, Medimate [9], which can measure lithium levels in blood. The Medimate is an example of a point-of-care (POC) device based on the micro total analysis system ( $\mu \mathrm{TAS})$ concept [10]. A $\mu$ TAS offers the possibility to integrate multiple procedures in a portable, low-cost platform which is simple to utilize without affecting the results. TDM of lithium with Medimate has been studied and recently deemed to be clinically useful [11] and is appropriate for self-testing [12]. While the Medimate device is an impressive and important development for the field, lithium is well-separated from other matrix components in the blood, and has a high therapeutic range, making detection relatively simple. Other $\mu$ TAS devices applicable to TDM have been developed for glutathione [13], creatinine [14], and $\beta$-hydroxybutyrate ( $\beta \mathrm{HB}$ ) [15].

One of the main impediments in developing a $\mu$ TAS is the complexity and size of the instrumentation required for the on-site analysis, and for at-home monitoring, it needs to be fully automated, simple to use, easy, and low-cost to make. Recently, Shallan et al. [16] developed a microfluidic device featuring two nanojunctions with different pore sizes to create a size and mobility trap (SMT) to purify and concentrate small molecules prior to an integrated electrophoretic separation. Significantly, the nanojunctions were created by dielectric breakdown after the device containing easier-to-fabricate micron-sized channels was bonded, providing a simpler pathway to commercial manufacture. The potential of the device for TDM was demonstrated with the determination of the antibiotic ampicillin from whole blood within $5 \mathrm{~min}$. However, this device was fabricated in Polydimethylsiloxane (PDMS) where large-volume production is not possible through hot embossing or injection molding. Li et al. [17] developed a similar device incorporating two commercially manufactured nanoporous membranes for the concentration and monitoring of albumin in urine as a marker of albuminurea. While functional, the pore sizes were too large for small molecule pharmaceuticals, and the additional fabrication steps to incorporate the two different membranes would significantly increase the cost of the device.

Here, a microfluidic device with an SMT feature created by dielectric breakdown was fabricated in PMMA instead of PDMS because of the well-known ability of hot embossing or injection molding in this material [18]. We demonstrate that similar nanojunctions can be created in PMMA using controlled dielectric breakdown and that a device with two different sized nanojunctions can be used for the analysis of aminoglycoside antibiotic drugs in whole blood.

\section{Experimental Section}

\subsection{Materials and Chemicals}

All solutions were prepared using Milli-Q water (18 M $\Omega$, Millipore, North Ryde, Australia). PDMS (Sylgard 184) elastomer and curing agent were purchased from Dow Corning (Michigan, MI, USA). Potassium thiocyanate (KSCN) was purchased from AJAX Chemicals (Sydney, Australia). 5(6)-Carboxy-X-rhodamine (ROX), R-phycoerythrin (RPE), fluorescein, fluorescamine, bovine serum albumin (BSA), Ferric chloride $\left(\mathrm{FeCl}_{3}\right)$, aminoglycoside antibiotics (gentamicin, amikacin and tobramycin), hexadimethrine bromide (HDMB), hydroxypropyl methylcellulose (HPMC), sodium tetraborate, di-sodium hydrogen phosphate, and sodium phosphate monobasic for buffer preparation were purchased from Sigma-Aldrich (Sydney, Australia). 


\subsection{Sample Preparation}

Fluorescamine was prepared in acetone to obtain $3 \mathrm{mg} / \mathrm{mL}$ as a stock solution. BSA was prepared in Milli-Q water at a concentration of $2 \mathrm{mg} / \mathrm{mL}$ and then labelled with fluorescamine at a ratio 3:1 in borate buffer at $\mathrm{pH}$ 9. ROX and fluorescein were prepared in Milli-Q water to get $25 \mu \mathrm{g} / \mathrm{mL}$ and $200 \mu \mathrm{g} / \mathrm{mL}$ solutions, respectively. $\mathrm{KSCN}$ and $\mathrm{FeCl}_{3}$ were prepared in Milli-Q water to obtain $2.5 \mathrm{M}$ and $25 \mathrm{mM}$, respectively. Aminoglycosides antibiotics (gentamicin, amikacin, and tobramycin) stock solutions were prepared in Milli-Q water at a concentration of $500 \mu \mathrm{g} / \mathrm{mL}$. These were then labelled with fluorescamine at a ratio of 3:1 in borate buffer at $\mathrm{pH}$ 9. Whole blood samples from a healthy volunteer were collected under the guidelines by the Tasmanian Health and Medical Human Research Ethics Committee, Office of Research Services, University of Tasmania (Ethics Approval Ref is H0016575) and spiked with aminoglycosides drugs (amikacin, gentamicin, and tobramycin) (stock solution) in a ratio of 10:0.1 to obtain different concentrations $(2,5,10,15,20 \mu \mathrm{g} / \mathrm{mL})$. The mixture was then labelled with $20 \mu \mathrm{L}$ fluorescamine in $20 \mu \mathrm{L}$ borate buffer $\mathrm{pH}=9$.

\subsection{Fabrication of Microfluidic Device}

All microfluidic devices were created on a $75 \mathrm{~mm} \times 50 \mathrm{~mm} \times 1.5 \mathrm{~mm}$ piece of PMMA (RS Components, Melbourne, Australia) using the following procedures, with a schematic shown in Figure S1. First, the negative template of the device was fabricated in SU-8 using previously described processes $[19,20]$. Then, PDMS mixed at 5:1 $(\mathrm{w} / \mathrm{w})$ was degassed, placed on the SU-8 template, and then heated in an oven at $70{ }^{\circ} \mathrm{C}$ for at least $12 \mathrm{~h}$. The positive PDMS stamp was then removed from the PMMA template and thermally aged in an oven at $250{ }^{\circ} \mathrm{C}$ for $30 \mathrm{~min}$. The aged PDMS template was used to hot emboss the PMMA channel plates $(1.5 \mathrm{~mm} \times 50 \mathrm{~mm} \times 75 \mathrm{~mm})$ as follows: the blank PMMA plate and PDMS positive master were placed between two $100 \mathrm{~mm} \times 100 \mathrm{~mm} \times 6 \mathrm{~mm}$ glass plates and put into a hot embosser (MTP-8, Tetrahedron, San Diego, CA, USA). Four steps were used in the embossing process: Step 1: temperature increased to $130^{\circ} \mathrm{C}$ at a rate of $92{ }^{\circ} \mathrm{C} / \mathrm{min}$, at a pressure of $100 \mathrm{lbs}$; Step 2: increasing the pressure up to $380 \mathrm{lbs}$ at a rate of $75 \mathrm{lbs} / \mathrm{min}$ at the temperature of $130{ }^{\circ} \mathrm{C}$, and hold for $20 \mathrm{~min}$; Step 3: the temperature was reduced at a rate of $15^{\circ} \mathrm{C} / \mathrm{min}$ until $60^{\circ} \mathrm{C}$ at $380 \mathrm{lbs}$; Step 4: the pressure was released, and the embossed device removed. The hot embossed PMMA device was then sealed with single-sided adhesive tape (Tesa SE, Charlotte, NC, USA), with the device's microchannels placed downward on $1 \mathrm{~mm}$ thick stainless steel plates. The assembly was then fed into the officer laminator (Peach 3500, Peach, Switzerland) at a $20^{\circ} \mathrm{C}$ temperature, speed 5 for four passes, with 90 degree clockwise rotation at each pass.

\subsection{Creation of Nanojunctions by Controlled Dielectric Breakdown}

The microfluidic device is hybrid PMMA/adhesive tape with the complete microchip shown in Figure 1a and a zoomed in image of the offset double V in Figure 1b. All microchannels had a $50 \mu \mathrm{m}$ depth. The separation channel was $50 \mu \mathrm{m}$ wide and the V channels were $500 \mu \mathrm{m}$. The hot embossed double-V PMMA device was used to create the SMT through the formation of nanojunctions in the $100 \mu \mathrm{m}$ gap between the tips of the V-channels and the separation channel. This gap distance was selected instead of $20 \mu \mathrm{m}$ [21] or $40 \mu \mathrm{m}$ [22] reported in the literature, to allow for a lengthened use of higher voltages during the extraction and purification stages without inducing secondary breakdown. The $500 \mu \mathrm{m}$ offset distance between the tips of the Vs permitted the two nanojunctions to connect and generate the SMT. The separation channel length was $60 \mathrm{~mm}$ and the effective length from the first nanojunction (injection point) to the detection point was $30 \mathrm{~mm}$. 
a)

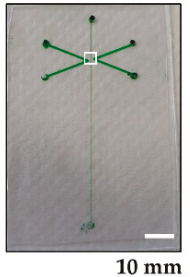

c)

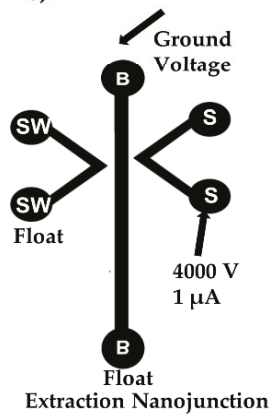

d)

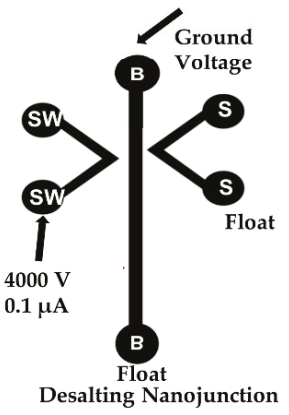

b)

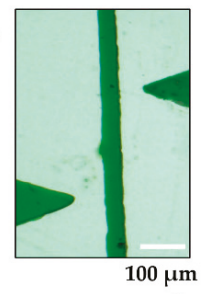

e)

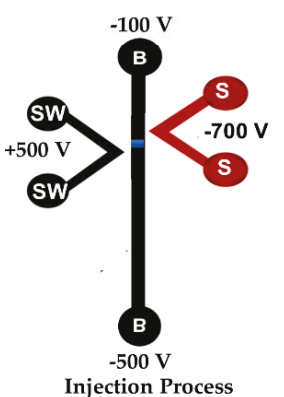

f)

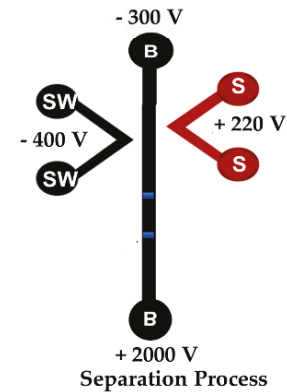

Sample

Background Electrolyte (BGE)

Figure 1. (a) Photograph image of the double-V hybrid PMMA/adhesive tape device filled with green food dye. Scale bar $=10 \mathrm{~mm}$. (b) Zoomed in microscope image of the offset double-V channel filled with green food dye (white box in panel a). Scale Bar $=100 \mu \mathrm{m}$. (c) Schematic of the microfluidic device (dimension not to scale) indicating voltages and terminating current used for generation of the extraction nanojunction and (d) the desalting nanojunction. (e) Injection voltages (60 s) to extract, concentrate, and desalt injected plug (blue) of small molecules (ROX and fluorescein). (f) Separation voltages (180 s) for electrophoretic separation. Buffer (B), Buffer Waste (BW), Sample (S), and Sample Waste (SW).

To make the nanojunctions, the separation channel and V-channel sample (S) were filled with the breakdown electrolyte, consisting of $10 \mathrm{mM}$ phosphate buffer, $\mathrm{pH} 11$, while the V-channel sample waste (SW) was filled with $100 \mathrm{mM}$ phosphate buffer, $\mathrm{pH}$ 7. The nanochannels were created by applying a high voltage of $4000 \mathrm{~V}$ over $100 \mu \mathrm{m}(40 \mathrm{~V} / \mu \mathrm{m}$, which is above the dielectric strength of PMMA $36 \mathrm{~V} / \mu \mathrm{m}$ [23]) to the V-sample (S) channel or sample waste (SW) channel whilst the separation channel was kept grounded, as shown in Figure 1c,d. The current limit was varied from $0.1 \mu \mathrm{A}$ to $5 \mu \mathrm{A}$ by utilizing an in-house adjustable power supply, controlled by the LabView HV V.6 program (National Instrument, Austin, TX, USA), which terminated the voltage once the current limit was achieved. The current limits were chosen to allow the transport of different sized molecules. The V-channels and the separation channel were then refilled with the experimental solutions after cleaning using Milli-Q water.

\subsection{Microdevice Operation}

After nanojunction creation, the sample V-channel (S) was filled with sample solution using an autopipette. The separation channel was filled with $100 \mathrm{mM}$ phosphate buffer, $0.5 \%$ HPMC, or $0.1 \%$ of $\mathrm{HDMB}$ at $\mathrm{pH}$ 11.5, while the V-channel sample waste (SW) was filled with $10 \mathrm{mM}$ phosphate buffer, $\mathrm{pH}$ 11.5, similar to those reported by Shallan et al. The extraction and separation voltages were applied using external electrodes for each reservoir, as shown in Figure 1e,f.

Visualization of ion enrichment/depletion was conducted using a Nikon high-definition colour CCD camera head (Digital Sight DS-Fi1c, Nikon, Japan) operated with NIS-Elements BR 3.10 software 
(Melville, NY, USA) mounted on an inverted fluorescence microscope (Ti-U, Nikon, Tokyo, Japan). Multiband pass excitation $\left(\lambda_{\mathrm{ex}}\right.$ at $390,482,563$, and $\left.640 \mathrm{~nm}\right)$ and emission $\left(\lambda_{\mathrm{em}}\right.$ at 446, 523, 600, and $677 \mathrm{~nm}$ ) filters (Semrock, Rochester, NY, USA) were used for all the experiments. A photomultiplier tube (PMT) (Hamamatsu Photonics KK, Hamamatsu, Japan) was used to perform the quantitative measurements which was connected to the microscope. An Agilent interface (35900E) connected to a laptop and operated by Agilent ChemStation for LC software (Agilent Technologies, Waldbronn, Germany) was used for data acquisition. An in-house four-channel $(0-5 \mathrm{kV})$ dc power supply was used to apply an electrical potential to each reservoir through a custom designed interface connected to six platinum electrodes.

\section{Results and Discussions}

In our previous work, we designed and demonstrated a new electrokinetic device with a SMT for the concentration and extraction of drugs, followed by their separation by electrophoresis. Electrophoresis is a separation mode in which ions, molecules, and particles are separated in an electric field in a conducting liquid medium. The speed of separation can be manipulated by variation of the liquid media composition, but is generally dependent on the charge and size of the ion/particle. Electrophoresis is known to be simpler than liquid chromatography, the other most common separation approach for TDM, and more easily miniaturized for portability. Here, we build on our previous electrokinetic sample treatment strategy, and implement it in a more manufacturable material.

\subsection{Nanojunction Creation and Transport Properties}

Previously, Shallan et al. demonstrated the ability to make nanojunctions of different sizes in PDMS by dielectric breakdown with termination currents from 1-5 $\mu \mathrm{A}$ [16,24]. Different charge and size analytes, such as labelled proteins $(4-10 \mathrm{~nm})$, aromatic compounds $(0.5-1.0 \mathrm{~nm})$, and small inorganic ions (effective hydrated radii of about $0.3 \mathrm{~nm}$ ) [25], were used to understand whether there were differences when compared to the previous work in PDMS. The results from these experiments are summarized in Figure 2.

A single nanojunction was formed after the breakdown using different current limits. When the termination current was set at $5 \mu \mathrm{A}$ (Figure 2 top row), the resulted nanojunctions blocked blood cells $(6-8 \mu \mathrm{m})$ [24] and RPE ( 10 $\mathrm{nm}$ in size) [17] from entering the separation channel, whilst electrophoretic transport of BSA labelled-fluorescamine $(2-4 \mathrm{~nm})$ into the separation channel was observed, with similar results obtained at $3 \mu \mathrm{A}$ (Figure 2 second row). Reducing the termination current to $1 \mu \mathrm{A}$ (Figure 2 third row) blocked the BSA, but allowed the transport of anionic ROX, fluorescein, and drugs. Previously, the movement of BSA was restricted in PDMS nanojunctions created with a termination current of $3 \mu \mathrm{A}$ [24], higher than what was achieved in this work. The different behavior in the PMMA devices may be related to the difference in surface charge and/or the elasticity between the two materials. To produce smaller channels, the ionic strength of the breakdown electrolyte was increased from $10 \mathrm{mM}$ phosphate to $100 \mathrm{mM}$ phosphate. A termination current of $0.1 \mu \mathrm{A}$ resulted in restricting the transport of BSA, ROX, fluorescein, and drugs, while still transporting small ions (thiocyanate) $(\sim 0.3 \mathrm{~nm})$ (Figure 2 bottom row). Overall, based on the permeability results of different sized molecules (Blood cell 4-6 $\mu \mathrm{m}$, RPE $\sim 10 \mathrm{~nm}$, BSA 2-4 nm, ROX $1 \mathrm{~nm}$, inorganic ions $0.3 \mathrm{~nm}$ ), we were able to estimate the size of fabricated nanochannels with this method.

\subsection{SMT Implementation}

The SMT is based on the preferential electrokinetic transport of ions through the resulting nanojunctions. The extraction nanojunction was formed by applying a $1 \mu \mathrm{A}$ termination current using $10 \mathrm{mM}$ phosphate $\mathrm{pH}=11$ electrolyte buffer as this allows small ions transport (e.g., ROX) through the free transport region, while restricting cells and plasma proteins transport (e.g., BSA). The concentration nanojunction between the separation channel and the V-sample channel was formed 
with a termination current of $0.1 \mu \mathrm{A}$ using $100 \mathrm{mM}$ phosphate $\mathrm{pH}=7$ as this prevents the transport of target analytes (e.g., ROX), but permits the small inorganic ions (e.g., Thiocyanate).

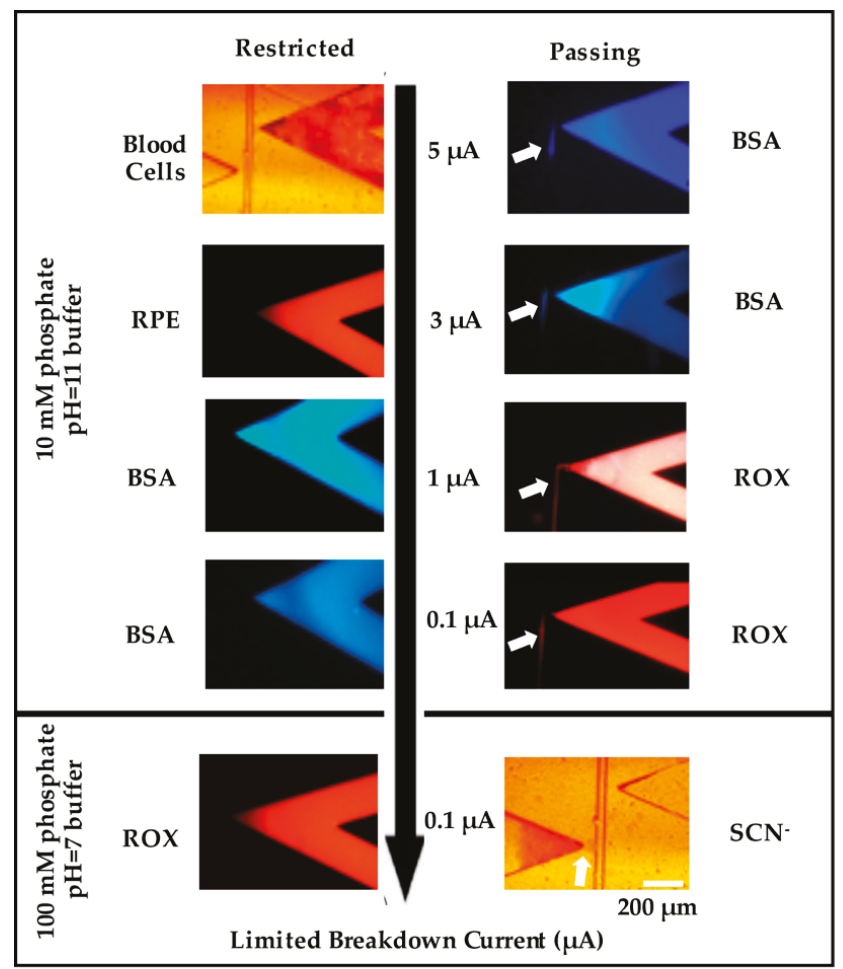

Figure 2. Screen shots showing the transport (right column) and restriction (left column) of different molecules through nanochannels created under different conditions. The top row shows the restriction of Blood Cells (left) and transport of BSA (blue, right); the second row shows the restriction of PDMS (red, left) and the transport of BSA (right); the third row shows the transport of ROX (right, Red) and restriction of BSA (left); the fourth row shows the restriction of BSA (left) and transport of ROX (right); the fifth row shows the restriction of ROX (left) and transport of thiocyanate (left). The first four rows used nanochannels created using a breakdown electrolyte of $10 \mathrm{mM}$ phosphate buffer, $\mathrm{pH}=11$, and different terminating currents $(5,3,1$, and $0.1 \mu \mathrm{A})$, while the fifth row used a breakdown electrolyte of $100 \mathrm{mM}$ phosphate buffer, $\mathrm{pH}=7$, and a terminating current of $0.1 \mu \mathrm{A}$. Images on the right show permeability (arrow), while those on the left show blocked transport. Scale Bar $=200 \mu \mathrm{m}$.

Figure 3 presents the ability and efficacy of the SMTs to extract and concentrate multiple small molecules. Figure $3 \mathrm{a}$ is a photo taken during the extraction and concentration of fluorescein (MW 332 Da) and ROX (MW 534.60 Da) in the separation channel. Figure 3b shows the separation of fluorescein and ROX immediately after switching the voltages from extraction and concentration to separation. $3 \mathrm{C}$ is the fluorescence trace collected using a PMT positioned $30 \mathrm{~mm}$ from the double-V intersection. These experiments show highly efficient separations of both fluorescein and ROX, with efficiencies of 414,000 and 203,000 plates $/ \mathrm{m}$, respectively. The relative standard deviation $(\mathrm{RSD} \%)=11.5 \%$ for fluorescein and $4.5 \%$ for $\operatorname{ROX}$ ( $n=3$ devices). 
a)

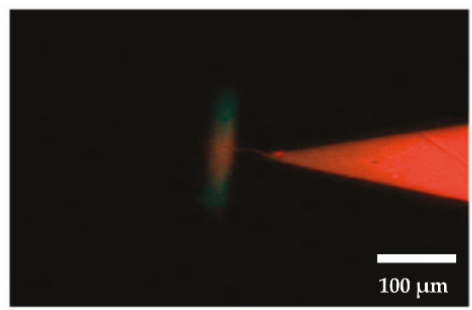

c)

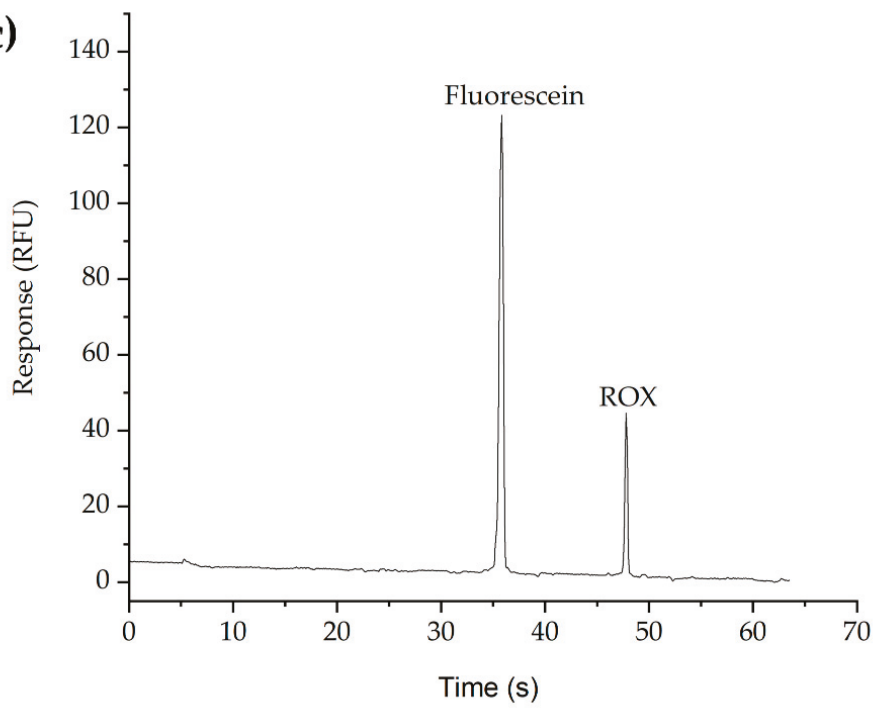

Figure 3. Screen shots showing (a) extraction, trapping, and (b) separation. Scale Bar $=100 \mu \mathrm{m}$. (c) Detection of small molecules (fluorescein and ROX). Current limit was set at $1 \mu \mathrm{A}$ for the right side and $0.1 \mu \mathrm{A}$ for the left side. BGE in the separation channel is $100 \mathrm{mM}$ phosphate buffer, $\mathrm{pH} 11.5$, with $0.5 \%$ HPMC to suppress the EOF and $0.1 \%$ HDMB to reverse the EOF, while in the left V-sample waste channel, $10 \mathrm{mM}$ phosphate buffer, $\mathrm{pH}$ 11.5, was used without HPMC. Applied voltages were set at $-100,-700,-500$, and $+500 \mathrm{~V}$ (for the injection) and $-300,+220,+2000$, and $-400 \mathrm{~V}$ (for the separation) for reservoirs B, S, BW, and SW, respectively.

\subsection{Extraction and Analysis of Aminoglycosides in Whole Blood}

The purpose of the SMT is to purify and concentrate anionic pharmaceuticals with a low molecular weight $(<1000 \mathrm{Da})$ from biological fluids such as blood. The applicability of the PMMA-embossed device for achieving this was demonstrated by the detection of the three aminoglycoside drugs gentamicin, amikacin, and tobramycin. after derivatization with fluorescamine using both the double- $\mathrm{V}$ device and a normal cross channel design with pinched injection [26]. Figure 4 shows the separation of a standard mixture of these three labeled aminoglycosides in both the double- $V$ device and a normal cross-design with pinched injection. It can be seen from the figure that the peaks in the double- $\mathrm{V}$ design are significantly higher than in the cross-device, demonstrating the concentration ability of the device. A 30-fold enhancement factor was achieved without any significant broadening of the peaks. 


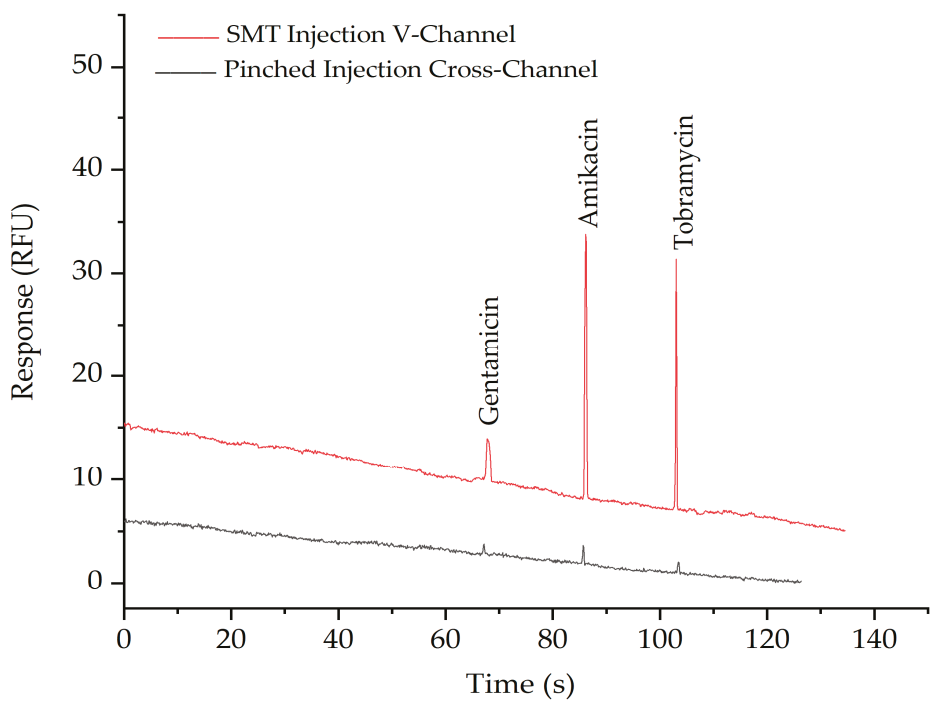

Figure 4. Electropherograms show the comparison of SMT (red trace) with pinched injection (black trace) of 5 ppm Gentamicin, 20 ppm Amikacin, and 10 ppm Tobramycin after labelling with fluorescamine. The BGE in the V- sample waste channel was $10 \mathrm{mM}$ phosphate buffer, $\mathrm{pH} 11.5$, while in the separation channel, it was $100 \mathrm{mM}$ phosphate buffer, $\mathrm{pH} 11.5$, with $0.5 \%$ HPMC. Applied voltages for SMT were $-100,-700,-500$, and $+500 \mathrm{~V}$ for $60 \mathrm{~s}$ and for separation were $-300,+220$, +2000 , and $-400 \mathrm{~V}$ at reservoirs B, S, BW, and SW, respectively. For pinched injection (cross-channel), all channels were filled with $100 \mathrm{mM}$ phosphate buffer, $\mathrm{pH} 11.5$, with $0.5 \%$ HPMC. Applied voltages for injection were $-100,-400,-300$, and $+400 \mathrm{~V}$ at B, S, BW, and SW reservoirs, respectively, and separation voltages were $-200,+400,+1500$, and +400 V at B, S, BW, and SW reservoirs, respectively.

The other function of the SMT is to purify the sample. This can be seen in Figure 5 and Figure S2a, which show the separation of blood spiked with the aminoglycosides and then labelled with fluorescamine. The cross device provides a clean peak for tobramycin, but interferences are observed for gentamicin and amikacin. This is due to fluorescamine reacting with all primary amines, including those on drugs, proteins, amino acids, etc., found in the blood. In contrast, the double-V device containing the SMT provides clean separations due to the removal of some of the interferences, most likely the larger proteins. Some endogenous small molecules are still observed in the SMT, but these are well-resolved from the target drugs in this instance, allowing full and complete quantitation of the three aminoglycosides. The device shows high recovery efficiencies for the three drugs (Gentamicin 92\%, Amikacin 98\%, and Tobramycin 98\%) and there were no clogging cases observed during the analysis.

The linear calibration curve of the Aminoglycosides (gentamycin, amikacin, and tobramycin) from blood is shown in Figure S2b. The LOD (signal to noise level of three) was $3.75 \mu \mathrm{g} / \mathrm{mL}$ for gentamicin, $8.53 \mu \mathrm{g} / \mathrm{mL}$ for amikacin, and $6 \mu \mathrm{g} / \mathrm{mL}$ for tobramycin, and they are linear to $2-20 \mu \mathrm{g} / \mathrm{mL}$, which covers the therapeutic range for treating sepsis of $6-10 \mu \mathrm{g} / \mathrm{mL}$ gentamicin and tobramycin and $12-20 \mu \mathrm{g} / \mathrm{mL}$ of amikacin [27].

In comparison to current methods for these aminoglycosides, they can be analyzed by immunoassays, such as the Fluorescence Polarization Immunoassay (FPIA), Radioimmunoassay (RIA), and Enzyme-linked Immunosorbent assay (ELISA). These assays have limitations. The FPIA assay has a good reproducibility, is comparatively cheap, and is labor non-intensive [27]. Additionally, FPIA is fully automated, but demands an IMx FPIA analyzer [28] that is relatively expensive. RIA was successfully used to determine the aminoglycosides in biological samples, but it has problems related 
to handling radioactivity and the equipment is costly [29]. The ELISA system labeling with primary antibodies is time-consuming and the labeling of the primary antibody from one experiment to another is not flexible [30]. HPLC (High Performance Liquid Chromatography) is a sensitive method for conducting the aminoglycosides, but requires expensive instruments [31] and shortening the separation time is required [32]. By Higher Performance Liquid Chromatography (HPLC), the LOQ for tobramycin is as low $0.05 \mu \mathrm{g} / \mathrm{mL}$ using $200 \mu \mathrm{L}$ of plasma [33]; however, these low levels are not necessary given the therapeutic range of aminoglycosides. When monitoring preterm infants, it is more profitable to use smaller volumes of sample, which should not overtake $75 \mu \mathrm{L}$ [34]. Here, the suggested assay demands $20 \mu \mathrm{L}$ of blood to give results within $3 \mathrm{~min}$.

Analysis of Aminoglycosides in Whole Blood

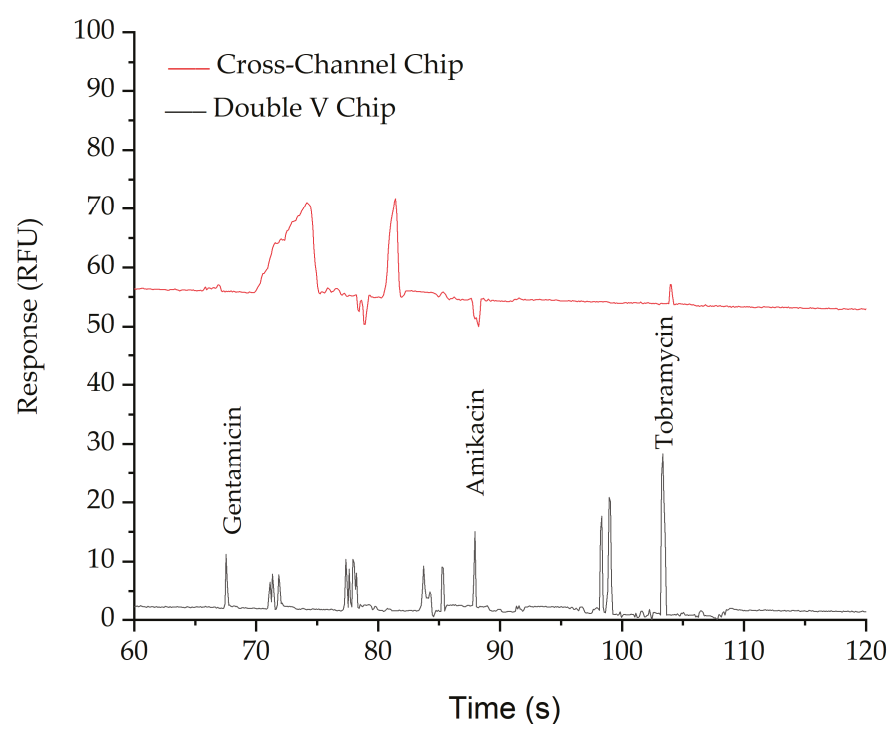

Figure 5. Electropherograms show the purification of whole blood spiked with $10 \mathrm{ppm}$ of gentamicin, amikacin, and tobramycin and derivatized with fluorescamine using a cross-chip (red trace) and the Double V- chip (black trace). The BGE in the V-sample waste channel was $10 \mathrm{mM}$ phosphate buffer, $\mathrm{pH} 11.5$, while in the separation channel, it was $100 \mathrm{mM}$ phosphate buffer, $\mathrm{pH} 11.5$, with $0.5 \%$ HPMC. Applied voltages for injection were $-100,-700,-500$, and $+500 \mathrm{~V}$ for $60 \mathrm{~s}$ and for separation were -300 , $+220,+2000$, and $-400 \mathrm{~V}$ at reservoirs B, S, BW, and SW, respectively. For cross-channel, all channels were filled with $100 \mathrm{mM}$ phosphate buffer, $\mathrm{pH}$ 11.5, with 0.5\% HPMC. Applied voltages for injection were $-100,-400,-300$, and $+400 \mathrm{~V}$ at $\mathrm{B}, \mathrm{S}, \mathrm{BW}$, and SW reservoirs, respectively, and separation voltages were $-200,+400,+1500$, and $+400 \mathrm{~V}$ at B, S, BW, and SW reservoirs. respectively.

\section{Conclusions}

We have demonstrated an integrated hot embossed PMMA featuring an SMT device for desalting, on-chip extraction, and concentration. The hot embossed PMMA controlling dielectric breakdown enabled nanojunctions' pore size tuning. This method permitted the analysis of aminoglycosides as negatively charged drugs which were off-chip spiked with on-site finger prick blood $(\sim 20-100 \mu \mathrm{L})$ and labelled with fluorescamine. The nanojunctions created using dielectric breakdown are achieved simply and quickly, and the electric field controlling pore size permits their integration without increasing the fabrication cost. The device allows a simple operation and does not rely on valves or pumps. With integrated reagents (dried fluorescamine and liquid electrolytes) and a hand-held reader, this would create a fully-portable screening device for POC analysis of therapeutics. 
Supplementary Materials: The following are available online at http://www.mdpi.com/2079-6374/9/1/19/s1. Figure S1: Schematic of the PMMA fabrication process. Figure S2: Analysis of Aminoglycosides from whole blood.

Author Contributions: Conceptualization, Z.T.A. and M.C.B.; Experiments and data analysis Z.T.A., Y.C.Y. and F.L.; Writing-Original Draft Preparation, Z.T.A.; Writing-Review \& Editing, Y.C.Y., F.L. and M.C.B.; Supervision and Project Administration, M.C.B.; Funding Acquisition, M.C.B.

Funding: This research was funded by The Australian Research Council (ARC) grant number [FT130100101].

Acknowledgments: Z.T.A. would like to acknowledge the Iraqi ministry of higher education and scientific research (MOHESR) and the Iraqi cultural attaché in Canberra for the study funding.

Conflicts of Interest: The authors declare no conflict of interest.

\section{References}

1. Lu, Y.-F.; Goldstein, D.B.; Angrist, M.; Cavalleri, G. Personalized medicine and human genetic diversity. Cold Spring Harb. Perspect. Med. 2014. [CrossRef] [PubMed]

2. Johannessen, S.I.; Battino, D.; Berry, D.J.; Bialer, M.; Krämer, G.; Tomson, T.; Patsalos, P.N. Therapeutic drug monitoring of the newer antiepileptic drugs. Ther. Drug Monit. 2003, 25, 347-363. [CrossRef] [PubMed]

3. Patsalos, P.N.; Berry, D.J.; Bourgeois, B.F.; Cloyd, J.C.; Glauser, T.A.; Johannessen, S.I.; Leppik, I.E.; Tomson, T.; Perucca, E. Antiepileptic drugs-Best practice guidelines for therapeutic drug monitoring: A position paper by the subcommission on therapeutic drug monitoring, ILAE Commission on Therapeutic Strategies. Epilepsia 2008, 49, 1239-1276. [CrossRef] [PubMed]

4. Rousseau, A.; Marquet, P. Application of pharmacokinetic modelling to the routine therapeutic drug monitoring of anticancer drugs. Fundam. Clin. Pharmacol. 2002, 16, 253-262. [CrossRef] [PubMed]

5. Haouala, A.; Zanolari, B.; Rochat, B.; Montemurro, M.; Zaman, K.; Duchosal, M.A.; Ris, H.B.; Leyvraz, S.; Widmer, N.; Decosterd, L.A. Therapeutic drug monitoring of the new targeted anticancer agents imatinib, nilotinib, dasatinib, sunitinib, sorafenib and lapatinib by LC tandem mass spectrometry. J. Chromatogr. $B$ 2009, 877, 1982-1996. [CrossRef] [PubMed]

6. Yu, H.; Steeghs, N.; Nijenhuis, C.M.; Schellens, J.H.; Beijnen, J.H.; Huitema, A.D. Practical guidelines for therapeutic drug monitoring of anticancer tyrosine kinase inhibitors: Focus on the pharmacokinetic targets. Clin. Pharmacokinet. 2014, 53, 305-325. [CrossRef] [PubMed]

7. Huttner, A.; Harbarth, S.; Hope, W.W.; Lipman, J.; Roberts, J.A. Therapeutic drug monitoring of the $\beta$-lactam antibiotics: What is the evidence and which patients should we be using it for? J. Antimicrob. Chemother. 2015, 70, 3178-3183. [CrossRef]

8. Winter, W.E.; Sokoll, L.J.; Jialal, I. Handbook of Diagnostic Endocrinology, 2nd ed.; AACC Press: Washington, DC, USA, 2008.

9. Vrouwe, E.X.; Luttge, R.; Vermes, I.; Van Den Berg, A. Microchip capillary electrophoresis for point-of-care analysis of lithium. Clin. Chem. 2007, 53, 117-123. [CrossRef]

10. Manz, A.; Graber, N.; Widmer, H.Á. Miniaturized total chemical analysis systems: A novel concept for chemical sensing. Sens. Actuators B Chem. 1990, 1, 244-248. [CrossRef]

11. Touw, D.J.; Neef, C.; Thomson, A.H.; Vinks, A.A. Cost-effectiveness of therapeutic drug monitoring: A systematic review. Ther. Drug Monit. 2005, 27, 10-17. [CrossRef]

12. Staal, S.; Floris, J.; Lenk, S.; Staijen, E.; Muñoz, M.A.; Kohlheyer, D.; Eijkel, J.; van den Berg, A. A Prefilled, Ready-to-Use, Electrophoresis-Based Lab-on-a-Chip Device for Monitoring Ions in Blood and Urine. In Proceedings of the 14th International Conference on Miniaturized Systems for Chemistry and Life Sciences, Groningen, The Netherlands, 3-7 October 2010; pp. 2107-2109.

13. Shihabi, Z.K.; Friedberg, M.A. Analysis of small molecules for clinical diagnosis by capillary electrophoresis. Electrophoresis 1997, 18, 1724-1732. [CrossRef] [PubMed]

14. Muñoz, M.; Eijkel, J.; Floris, A.; Staal, S.; Ríos, A.; Van Den Berg, A. The Development of a Point of Care Creatinine Measurement Using Disposable Ready to Use Microchip Capillary Electrophoresis. In Proceedings of the 15th International Conference on Miniaturized Systems for Chemistry and Life Sciences, Seattle, WA, USA, 2-6 October 2011; pp. 2-6.

15. Shallan, A.; Guijt, R.M.; Breadmore, M.C. Microfluidic Devices for The Analysis of Drugs and Their Metabolites in Biological Fluids; University of Tasmania: Hobart, Australia, 2013. 
16. Shallan, A.I.; Guijt, R.M.; Breadmore, M.C. Electrokinetic Size and Mobility Traps for On-site Therapeutic Drug Monitoring. Angew. Chem. Int. Ed. 2015, 54, 7359-7362. [CrossRef] [PubMed]

17. Li, F.; Guijt, R.M.; Breadmore, M.C. Nanoporous membranes for microfluidic concentration prior to electrophoretic separation of proteins in urine. Anal. Chem. 2016, 88, 8257-8263. [CrossRef] [PubMed]

18. Grass, B.; Neyer, A.; Jöhnck, M.; Siepe, D.; Eisenbeiß, F.; Weber, G.; Hergenröder, R. A new PMMA-microchip device for isotachophoresis with integrated conductivity detector. Sens. Actuators B Chem. 2001, 72, 249-258. [CrossRef]

19. Guijt, R.M.; Breadmore, M.C. Maskless photolithography using UV LEDs. Lab Chip 2008, 8, 1402-1404. [CrossRef] [PubMed]

20. Breadmore, M.C.; Guijt, R.M. High intensity light emitting diode array as an alternative exposure source for the fabrication of electrophoretic microfluidic devices. J. Chromatogr. A 2008, 1213, 3-7. [CrossRef] [PubMed]

21. Kim, S.M.; Burns, M.A.; Hasselbrink, E.F. Electrokinetic protein preconcentration using a simple glass/poly (dimethylsiloxane) microfluidic chip. Anal. Chem. 2006, 78, 4779-4785. [CrossRef]

22. Lee, J.H.; Chung, S.; Kim, S.J.; Han, J. Poly (dimethylsiloxane)-based protein preconcentration using a nanogap generated by junction gap breakdown. Anal. Chem. 2007, 79, 6868-6873. [CrossRef]

23. Seela-or, S.; Tonmitr, K.; Kaewrawang, A. The Electrical Breakdown of PVC and PMMA Barrier in Oil Insulator under Non-Uniform Field. In Proceedings of the 2016 International Conference on Mechanics, Materials and Structural Engineering, Jeju Island, Korea, 18-20 March 2016; pp. 172-176.

24. Shallan, A.I.; Gaudry, A.J.; Guijt, R.M.; Breadmore, M.C. Tuneable nanochannel formation for sample-in/answer-out devices. Chem. Commun. 2013, 49, 2816-2818. [CrossRef]

25. Cervera, J.; Ramírez, P.; Manzanares, J.A.; Mafé, S. Incorporating ionic size in the transport equations for charged nanopores. Microfluid. Nanofluid. 2010, 9, 41-53. [CrossRef]

26. Jacobson, S.C.; Hergenroder, R.; Koutny, L.B.; Warmack, R.; Ramsey, J.M. Effects of injection schemes and column geometry on the performance of microchip electrophoresis devices. Anal. Chem. 1994, 66, 1107-1113. [CrossRef]

27. Begg, E.J.; Barclay, M.L.; Kirkpatrick, C.M. The therapeutic monitoring of antimicrobial agents. Br. J. Clin. Pharmacol. 2001, 52, 35-43. [CrossRef]

28. Donnelly, J.G.; Pronovost, C. Evaluation of the Abbott IMxTM fluorescence polarization immunoassay and the Bio-Rad enzyme immunoassay for homocysteine: Comparison with high-performance liquid chromatography. Ann. Clin. Biochem. 2000, 37, 194-198. [CrossRef] [PubMed]

29. Stead, D.A. Current methodologies for the analysis of aminoglycosides. J. Chromatogr. B Biomed. Sci. Appl. 2000, 747, 69-93. [CrossRef]

30. Crowther, J.R. Systems in ELISA. In ELISA Guidebook; Humana Press: New York, NY, USA, 2009; pp. 9-42.

31. Yang, Z.; Jiang, W.; Liu, F.; Zhou, Y.; Yin, H.; Ai, S. A novel electrochemical immunosensor for the quantitative detection of 5-hydroxymethylcytosine in genomic DNA of breast cancer tissue. Chem. Commun. 2015, 51, 14671-14673. [CrossRef] [PubMed]

32. Peristyy, A.; Nesterenko, P.N.; Das, A.; D'Alessandro, D.M.; Hilder, E.F.; Arrua, R.D. Flow-dependent separation selectivity for organic molecules on metal-organic frameworks containing adsorbents. Chem. Commun. 2016, 52, 5301-5304.

33. Attema-de Jonge, M.E.; Bekkers, J.M.; Oudemans-van Straaten, H.M.; Sparidans, R.W.; Franssen, E.J. Simple and sensitive method for quantification of low tobramycin concentrations in human plasma using HPLC-MS/MS. J. Chromatogr. B 2008, 862, 257-262. [CrossRef] [PubMed]

34. Koren, G. Therapeutic drug monitoring principles in the neonate. Clin. Chem. 1997, 43, 222-227. [PubMed] 

Review

\title{
Droplets for Sampling and Transport of Chemical Signals in Biosensing: A Review
}

\author{
Shilun Feng ${ }^{1,2, *}$, Elham Shirani ${ }^{1}$ and David W. Inglis ${ }^{1,2, *}$ \\ 1 School of Engineering, Macquarie University, Sydney, NSW 2109, Australia; \\ elham.shirani-faradonbeh@students.mq.edu.au \\ 2 ARC Centre of Excellence for Nanoscale BioPhotonics (CNBP), Macquarie University, Sydney, \\ NSW 2109, Australia \\ * Correspondence: shilun.feng@gmail.com (S.F.); david.inglis@mq.edu.au (D.W.I.); \\ Tel.: +61-2-9850-9144 (D.W.I.)
}

Received: 2 April 2019; Accepted: 17 June 2019; Published: 20 June 2019

\begin{abstract}
The chemical, temporal, and spatial resolution of chemical signals that are sampled and transported with continuous flow is limited because of Taylor dispersion. Droplets have been used to solve this problem by digitizing chemical signals into discrete segments that can be transported for a long distance or a long time without loss of chemical, temporal or spatial precision. In this review, we describe Taylor dispersion, sampling theory, and Laplace pressure, and give examples of sampling probes that have used droplets to sample or/and transport fluid from a continuous medium, such as cell culture or nerve tissue, for external analysis. The examples are categorized, as follows: (1) Aqueous-phase sampling with downstream droplet formation; (2) preformed droplets for sampling; and (3) droplets formed near the analyte source. Finally, strategies for downstream sample recovery for conventional analysis are described.
\end{abstract}

Keywords: microfluidic probe; droplet; sampling; Taylor dispersion

\section{Introduction}

Biosensors have broad applications in drug discovery, medical diagnostics [1,2], environmental monitoring and food safety [3]. The concentration of specific analytes and their reaction kinetics can be identified in biosensors [4,5]. Biosensors using microdroplets have offered reconfigurability and flexibility and limited contamination during sample preparation and analysis [4]. In this review, we focus on the use of droplets to improve the temporal, spatial and chemical resolution of biosensing measurements by compartmentalizing samples during transport from the sampling site, to the analytical site. In doing so, it is possible to reduce Taylor dispersion.

In 1953, Taylor dispersion was described as "the combined action of molecular diffusion and the variation of velocity over the cross section" [6]. Because of this dispersive phenomena, the distance that chemical signals (variation in concentration with time and space) or analytes can be transported in the continuous phase is severely limited [6,7]. The top part of Figure 1 shows pulses of imaginary chemical A and chemical B. Initially, the pulses are separated from one another, but after a short distance the chemical pulses have merged and spread out [8]. In contrast, the bottom part of Figure 1 [8] shows that pulses of chemical A and B, when contained in droplets are not dispersed or merged after transport. The sampling of tiny volumes of aqueous body fluids and their transport to systems for precise detection or quantification is an ongoing area of activity in biomedical research [9-25].

The chemical content of a sample taken from a biological system may change, depending on when and where the sample is taken. For example, changes in neurotransmitter concentrations in the extracellular space around synapses are known to happen in milliseconds to seconds [26-29]. It is also well known that several neurotransmitters coexist in a given synaptic region, and that they can be 
released at different times [28,29]. It has been shown by Bert [30] that glutamate changes occurring in 1 min can be completely dampened when samples are pooled, as opposed to discretized.

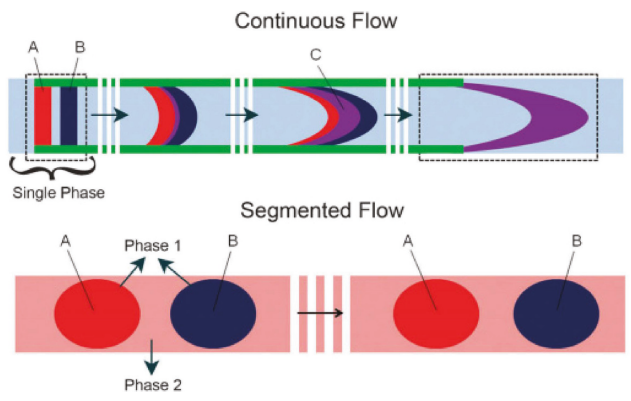

Figure 1. Schematic representation of using segmented flow to avoid the Taylor dispersion. (The vertical white lines represent the passage of space and time (Reprinted with permission from Reference [8]. Copyright 2011 Royal Society of Chemistry).

It is reported that temporal resolution for conventional high-performance liquid chromatography (HPLC) is minutes [31]. Such time scales make it impossible to quantify analyte concentrations that change rapidly accurately. Droplet-based approaches can be used to effectively avoid dispersion and improve temporal resolution by capturing and storing events that occur too quickly for a particular analytical method [32-37]. Microdialysis sampling, coupled with droplets and direct infusion mass spectrometry was used for acetylcholine monitoring [38] with intervals of just a few seconds. Monitoring of real-time streptavidin-biotin binding kinetics was also achieved using droplet microfluidics integrated with confocal spectroscopy [39]. Srinivasan et al. [40] reported the integration of optical absorbance measurements with droplet-based microfluidics for the detection of glucose using glucose oxidase in less than $40 \mathrm{~s}$. The coupling of a digital droplet-based microfluidic device to surface plasmon resonance (SPR) imaging has also been demonstrated [41,42].

Water-in-oil droplets are not perfect containers. Water and small molecules move through the water-oil interface at a non-zero rate [43]. Surfactants at the interface may form micelles, leading to another mechanism for analytes to escape droplets. Air bubbles have been used as separators between aqueous slugs to reduce cross contamination [44], and leakage is suspected to be worse in the corners of rectangular channels [45].

Conventional sampling tools, which can undertake a continuous sampling of body fluid, cannot sample and transport rapid changes of chemical signals from the insertion point to an analytical instrument without signal distortion, because of the Taylor dispersion phenomenon. Similarly, the same signal distortion problem also occurs when delivering sequences of different drugs to the injection point. A real-time analytical chemistry lab, small enough to fit inside the brain of a mouse does not exist. However, we can achieve similar analytical aims if we can digitize the liquid environment from precise locations within an organism at precise times using water-based liquids, carried by an immiscible oil in a hydrophobic channel. In this paper, we review state-of-the-art low-volume sampling probes that use droplets to transport signals for downstream analysis. For these sampling tools, hydrophobic and hydrophilic surfaces are used to control the movement of liquids.

\section{Theory}

\subsection{Taylor Dispersion}

Taylor dispersion acts to enhance diffusion, which can reduce the temporal and chemical resolution of biosensors [46-48], DNA analysis [49-51], mass spectrometry [52-54], surface patterning [55-58] and other applications. It results from the interaction of convection and diffusion within a pipe or channel. 
Convection is the transport of fluid axially in the flow direction. The typical Poiseuille laminar flow in a low Reynolds number channel has its maximum flow velocity in the centre of the channel and decreases smoothly to zero at the walls. Volumes of fluid near the centre of the channel will move much faster than those near the walls. A group of molecules, initially near one-another is, thus, spread out. Diffusion makes the problem worse. Diffusion is driven by a gradient in the chemical concentration of the diffusing species [59]. When considering a group of molecules forming a pulse of an analyte, the sharper the gradient constituting that pulse, the more rapidly it disappears.

The effective diffusion in a capillary was described by Sir Geoffrey Taylor and R Aris [60], it is as the sum of conventional axial diffusion $(D)$ and the Taylor dispersion coefficient. Together they are given an equation for the effective diffusion coefficient in a capillary known as the Taylor-Aris dispersion coefficient $[61,62]$ :

$$
D_{\text {eff }}=D\left(1+\frac{1}{48} P e_{d}^{2}\right),
$$

where $P e_{d}$ is the Peclet number, and is the ratio of convective fluxes to diffusive fluxes in the system. It can be defined as $P e_{d}=2 r \bar{V} / D$ where $r$ is the channel radius, and $\bar{V}$ is the average value of the velocity in the Poiseuille flow. Combining the two makes it clear that when the average velocity is greater than $2 \sqrt{3} D / r$, advection is the dominant cause of diffusion and dispersion. For a typical small molecule of Sucrose chemicals ( $D_{\text {Sucrose }}=500 \mu \mathrm{m}^{2} / \mathrm{s}$ ) in a 100-um-wide channel, Taylor dispersion dominates when the average velocity is above $17 \mu \mathrm{m} / \mathrm{s}$. This very low velocity highlights the futility of moving chemical signals in microchannels using continuous flow.

\subsection{Biosensing with a Chemical, Spatial and Temporal Resolution}

The sampling and delivery of tiny amounts of body fluids for accurate analysis is of great interest for fundamental biological studies, diagnostics, and therapeutics [22,25]. Specific and responsive signals, derived from the patient's body can help the study of fundamental biological processes, and optimize the use of medical therapies by allowing them to be more accurately dosed and more precisely targeted [63,64]. Effective droplet generation transport and analysis may allow advances in biosensing through improved chemical, spatial and temporal resolution. These, in turn, can be used for a wide range of applications.

An in vivo measurement system should be concerned with three parameters: Analytical performance, spatial resolution, and temporal resolution. Analytical performance refers to a variety of measurement parameters. These include the minimum concentration that can be detected (limit of detection), the smallest difference between two samples that can be resolved (resolution), and the ability of the measurement to respond solely to changes in the target analyte concentration (specificity or selectivity).

Spatial resolution is a well-understood concept in imaging, where the term voxel refers to a $3 \mathrm{D}$ volume over which the information is averaged. The same principle can be applied to chemical sampling, where higher spatial resolution gives more localized information. Given that chemicals can permeate surrounding tissue, and that signals produced in one place spread, and, therefore, decrease in concentration, we would also expect a higher spatial resolution to enable the detection of more rapid changes in analyte concentrations from smaller sources. The cross-section of the probe in contact with the tissue is a primary determinant of spatial resolution. However, the volume of sample extracted will also influence the spatial resolution. Drawing a large volume with a very fine probe will average a larger chemical voxel, than drawing a small volume with the same size probe [65-68].

Temporal resolution refers to the time taken for the measured value to change in response to a step-change in the sample. This change may be fit to a single exponential, which allows for an easily defined time constant [69]. Temporal resolution may also be defined as $t_{\text {res }}=\varnothing / f$, where $f$ is the sampling frequency or rate $(\mathrm{Hz})$, and $\varnothing$ is the number of plugs required to observe a change (from 10 to $90 \%$ of a concentration step) [70]; In another example [10], $\varnothing$ was defined as the number of plugs (or samples) needed to exchange $95 \%$ of molecules of interest. 
The response time of a complete system may also be limited by reaction kinetics at a sensor surface, but in this review, we are interested in the fundamental response time of the sampling process. This time delay is created by the movement of molecules from the signal source to the location of droplet break-up. Once in the droplets, we assume the droplet contents are fully mixed, and we ignore any further chemical reactions or changes that might occur inside or between the droplets. For a probe that extracts volume from the source, this time is approximately equal to the volume of liquid between source and droplet, divided by the volume flow rate. The temporal resolution can be reduced by extracting more fluid from the tissue, but this may damage or interfere with normal physiology.

For a probe that relies on diffusion across a membrane (microdialysis probes, for example), the speed of molecular transport is proportional to the analyte gradient and permeability of the membrane [71]. A device could sample a small percentage of the analyte with a high perfusion rate, achieving greater temporal resolution. However, capturing a high percentage of the source signal requires the concentrations on either side of the membrane to approach equilibrium, and, thus, a lower perfusion rate [72], and lower temporal resolution.

In a droplet system, we should consider the sampling rate and its relationship to the rate change of the thing being measured. Electrical engineers have addressed this problem through frequency analysis and arrived at a sampling theorem, which states that the sampling rate (samples per second) should be twice as fast as the fastest changing component of the signal, the Nyquist rate.

\subsection{Interface Forces}

Biological and chemical signals are typically generated in a continuous aqueous environment, and most analytical processes take the same fluid phase as inputs. Transporting signals from one location to another requires the digitizing of liquid packages at one end of the channel, analogous to an analog to digital conversion in electronics. At the receiving end of the channel, the reverse may happen. Here, the oil phase is removed in a process analogous to digital to analog (D2A) conversion. This packaging and unpackaging must overcome interfacial forces, the primary one being Laplace or bubble pressure. This pressure is controlled by three parameters: Surface tension, contact angle and hydraulic diameter.

Surface tension is the first parameter that can affect the Laplace pressure. It is defined pragmatically as: If a line is drawn on the surface of an interface, then one can determine the equilibrium state by assuming that the molecules on one side of the line exert a force $\tau$ per unit length of the line on the molecules on the other side. The $\tau$ will be the surface tension, and it is directed tangent to the surface [73]. Any work done against this force will increase the surface energy of the system. Fluid interfaces minimize their energy by taking shapes that minimize their surface area. These shapes can generate pressure differences across the interface. This pressure is referred to as the Laplace or bubble pressure and is given by the following equation [73]:

$$
\Delta \mathbf{P}=\boldsymbol{P}_{\text {inside }}-\boldsymbol{P}_{\text {outside }}=\tau\left(\frac{1}{\boldsymbol{R}_{1}}+\frac{1}{\boldsymbol{R}_{2}}\right),
$$

where $R_{1}$ and $R_{2}$ are the principal radii of curvature and $\tau$ is the surface tension of the aqueous/oil interface. For spherical droplets, $R_{1}$ and $R_{2}$ are the same. Therefore, the Laplace pressure can be defined as [73]:

$$
\Delta \mathbf{P}=\frac{2 \tau}{\mathbf{R}}
$$

For an interface that is bounded by a solid surface, such as a microchannel or membrane pore, the Laplace pressure will relate to the contact angle as [73]:

$$
\Delta \mathbf{P}=\frac{2 \tau}{\mathbf{R}^{\prime} \cos \theta}
$$

where $R^{\prime}$ is the hydraulic diameter of the structure containing the interface, and $\theta$ is the contact angle for the two fluids at the solid surface. This pressure must be overcome to create droplets, and it can be used, as we shall see later, to control the movement of certain phases in a two-phase system [74-78]. 


\section{Sampling Devices}

In this review paper, we survey examples where droplets have been applied to the delivery and/or sampling of chemical signals. The work is divided into three categories: Aqueous phase sampling with downstream droplet formation; preformed droplets for sampling; and droplets formed near the analyte source.

\subsection{Aqueous-Phase Sampling with Downstream Droplet Formation}

This category is characterized by an aqueous phase sampling probe that draws a sample from within the tissue, then transports it to a microfluidic device outside the tissue. The sampled fluid is then segmented at the external microfluidic devices. Methods of obtaining a sample that we consider here are: Diffusion through a membrane (dialysis probe) and direct fluid extraction (push-pull cannula, push-pull microfabricated sampling probe, hydrophilic capillary tube).

\subsubsection{Diffusion through a Membrane}

Microdialysis is widely used as the sampling probe for in vivo monitoring [79], clinical studies [80-82] and pharmacokinetics [83]. However, the drawback of this probe is that it has a large sampling surface. The membrane is typically over $2 \mathrm{~mm}$ long and more than $200 \mu \mathrm{m}$ in outer diameter. This large area limits spatial resolution.

A modern trend is to apply microdialysis in various clinical situations, such as monitoring concentrations of glucose, lactate, glutamate, and urea [84]. The microdialysis probe makes it possible for sampling to be done frequently without any loss of the volume from the tissue. Figure 2a shows a microdialysis probe coupled to a droplet generation chip to transport chemical signals to a distant capillary electrophoresis system $[11,85]$. The device has been used for sampling of neurotransmitter signals in a rat brain [86]. It has also been applied to an immobile live animal [87].

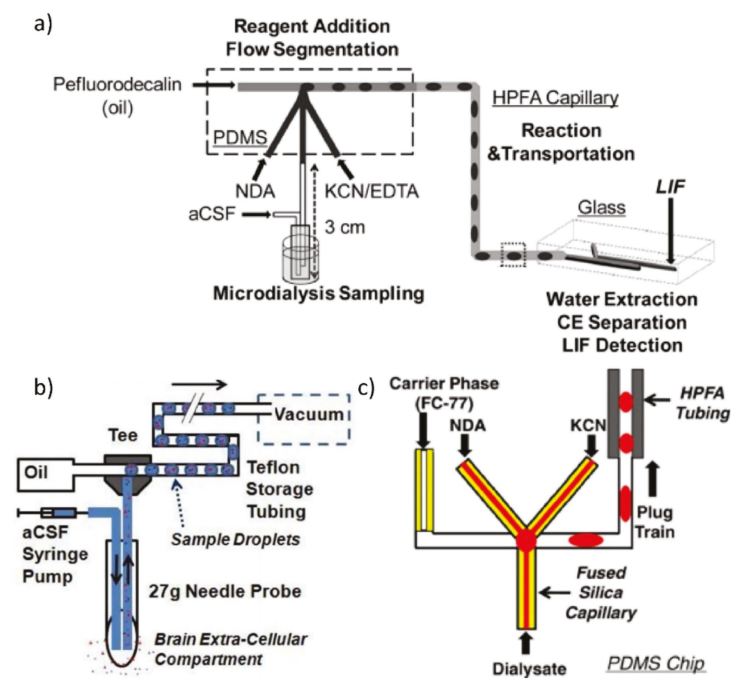

Figure 2. Continuous phase sampling with downstream droplet formation methods. (a) A microdialysis probe is used for sampling, coupled with a droplet generation chip to transport chemicals to a CE system (Reprinted with permission from Reference [85]. Copyright 2009 American Chemical Society); (b) research on low-flow push-pull probe system worked with a droplet system (Reprinted with permission from Reference [70]. Copyright 2011 American Chemical Society); (c) a capillary is used as the sampling probe, and attached to a droplet generator (Reprinted with permission from Reference [88]. Copyright 2011 Springer Nature). 


\subsubsection{Direct Fluid Extraction}

In this method, a buffer is continuously infused (pushed) into the tissue through one tube; while the sample is withdrawn (pulled) from a second tube that may be parallel or concentric with the infusion tube. These push-pull sampling systems are typically assembled by hand from capillary tubes and have been used in the brain since $1961[89,90]$. As above, the sample is segmented on a separate device that is a short distance downstream.

A temporal response of 5 min was achieved with low flow push-pull perfusion combined with off-chip fraction collection and analysis by capillary electrophoresis [68]. Lower temporal response of $16 \mathrm{~s}$ [91] and $45 \mathrm{~s}[92,93]$ was also achieved by coupling low-flow push-pull perfusion on-chip with CE for detection of samples from the eye and brain, respectively. Low-flow push-pull perfusion using a sampling probe with a smaller dead volume can be coupled with the segmented flow to achieve $7 \mathrm{~s}$ temporal response and spatial resolution of $0.016 \mathrm{~mm}^{2}$ in vivo [70] (Figure 2b). By further miniaturizing the probe inlet from $20 \mu \mathrm{m}$ to a $10 \mu \mathrm{m}$, and reducing the dead volume, the authors showed in vitro sampling with $200 \mathrm{~ms}$ response time [70]. Figure 2c shows a probe with a $0.5 \mathrm{~mm}$ probe combined with a downstream droplet generator [88].

Very recently, van den Brink et al. demonstrated a microfabricated silicon-based push-pull probe with a $1 \mathrm{~cm}$ long probe and integrated droplet generation structure [94]. This device showed a temporal resolution of a few seconds and a sampling area of just $0.004 \mathrm{~mm}^{2}$. The device was used to record the glutamate level in the sensorimotor cortex of a mouse brain experiencing targeted electrical stimulation.

\subsection{Preformed Droplets for Sampling}

This section summarizes sampling methods that use pre-formed droplets. Song et al. used a hydrophilic capillary tube to sample the changing concentration solution of $\mathrm{CaCl}_{2}(0.2-0.4 \mu \mathrm{L} / \mathrm{min}$, outside the chip. This hydrophilic capillary meets a hydrophobic channel carrying assay droplets. The sample is merged with the assay droplets for downstream analysis. The cross-section is $100 \mu \mathrm{m}$ by $100 \mu \mathrm{m}$, but the length of the sampling capillary is unclear [95]. This length of capillary will cause dispersion.

To solve the Taylor dispersion problem, Chen and Drew [96] and Chen et al. [10] brought droplets to the sampling site. Chen and Drew [96] proposed a microdialysis device where a pre-formed droplet passes a semi-permeable membrane. While on the membrane, analytes diffuse into the droplet. The droplet then moves downstream for analysis. D. Chen built a very similar system in the same year [10]. This approach, shown in Figure $3 \mathrm{a}-\mathrm{c}$, is used for sampling and/or introducing matter (stimulating) a planar environment, such as cell culture. A hydrophobic channel carrying oil is exposed on one side, to cells by clamping the device against a flat surface. Droplets are generated upstream of the interaction side. When those droplets reach the cells, they briefly make fluidic contact, exchange molecules, and are then carried away by the continuous oil phase.

The spatial resolution of the device was around $0.08 \mathrm{~mm}^{2}$ and was set by the size of the opening. In this work, the authors sampled at one droplet per second and showed $>95 \%$ change in signal in just two droplets. The volume of each slug was $30 \mathrm{~nL}$. The temporal response is not limited by Taylor dispersion because the sampling probe length is almost 0 (there is virtually no dead volume in the system). It should be noted that contamination of the tissue sample is quite possible as the channel was under positive pressure and had to be clamped to the tissue culture to prevent oil and buffer droplets from leaking into the tissue culture. 


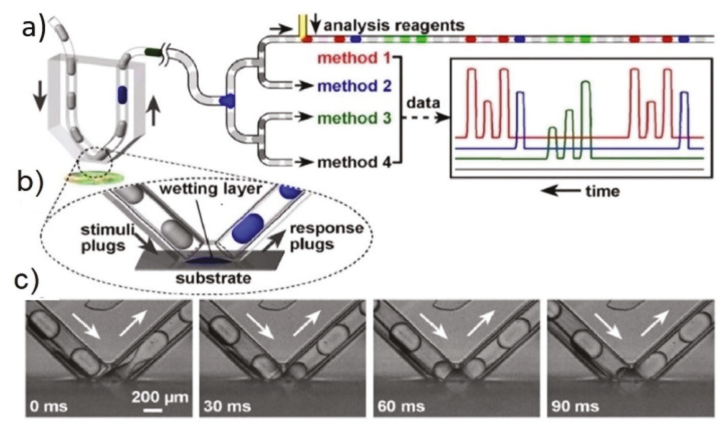

Figure 3. Pre-formed droplets are used to stimulate and extract analyte from a substrate, such as cell culture. (a) schematic of a device in operation; (b) an opening in the chip allows for droplet to merge with the hydrophilic substrate; (c) time-lapse bright-field images (side view) of the droplet extracting contents at $0 \mathrm{~ms}, 30 \mathrm{~ms}, 60 \mathrm{~ms}$ and $90 \mathrm{~ms}$. (Reprinted with permission from Reference [10]. Copyright 2008 National Academy of Sciences, USA).

\subsection{Droplets Formed near the Analyte Source}

Several researchers have developed methods that reduce the distance over which dispersion may occur by forming droplets very near to the signal source. We can further categorize the examples by considering whether a membrane or other barrier exists between the microfluidic channel and the tissue to be sampled.

\subsubsection{Droplets Formed near a Source without a Barrier}

Figure 4a shows a device that has three channels; negative pressure is applied to the middle channel, generating enough force to draw the sample into the device and form droplets. Other channels supply aqueous sheath flow and a continuous phase [97]. Figure $4 \mathrm{~b}$ illustrates a sampling probe made using a pipette tip and a concentric co-flowing oil. With carefully balanced pressures, droplets are formed at the tip and drawn into the Teflon tube [98]. MilliDrop (Paris France) is a commercial product that uses a similar approach, along with an air droplet to sample liquids from by dipping the tip in and out of the sample liquid.

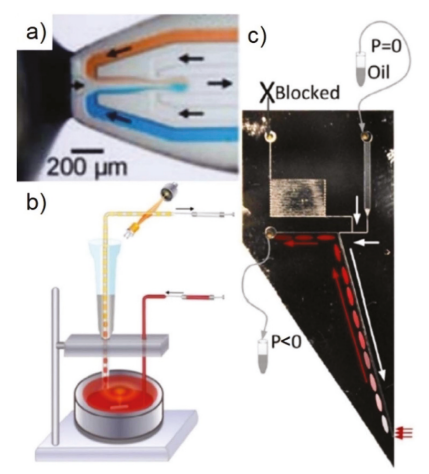

Figure 4. Methods that form droplets near the analyte source: (a) The middle channel is under negative pressure, sucking sample in through the device tip [97] (reprinted with permission. Copyright 2010 Royal Society of Chemistry); (b) droplet generated at the tip as co-flow. A Simple demonstration of sampling at the tip for 3-bromopropan-1-ol detection (reprinted with permission from Reference [98]. Copyright 2014 American Chemical Society); (c) sampling probe from Feng et al. which uses a microfabricated hydrophilic barrier (Reprinted with permission from Reference [69]. Copyright 2017 AIP Publishing LLC). 


\subsubsection{Droplets Formed at the Source with a Hydrophilic Barrier}

Examples included in this section have implemented some form of Laplace-pressure-barrier to allow water to pass in or out of a hydrophobic channel, while retaining the oil-based continuous phase. Laplace pressure, known as bubble pressure in membrane science, is the pressure difference across a curved liquid interface. It manifests as a barrier to the ingress of air or oil into an already wet hydrophilic membrane.

Our group has fabricated a silicon device for droplet-based sampling [69]. Figure 4c shows this device, which only has $2.8 \mathrm{pL}$ of dead volume between the channel and the exterior of the device. Hydrophilic structures, at the tip, contain this dead volume, and generate the surface tension barrier [69]. The device has been applied for on-site sampling and quantitative detection of Hydrogen Peroxide $\left(\mathrm{H}_{2} \mathrm{O}_{2}\right) \cdot \mathrm{H}_{2} \mathrm{O}_{2}$ samples were drawn into the device and immediately merged with assay droplets for reaction and downstream detection [99].

A hydrophilic membrane integrated within a planar microfluidic device has also been achieved. In this work, water in oil droplets is transported through a hydrophobic channel to a droplet exit port. The port is created by sealing a small section of the channel with a hydrophilic membrane. The wetted membrane resists penetration by the oil phase, but allows droplets to exit the channel [100]. Droplet delivery was demonstrated, but sampling was not.

\section{Droplet Extraction for Downstream Analysis}

A range of on-chip droplet-based detection and analysis methods are described in Qun Fang Group's review [101]. However, many sophisticated chemical analysis techniques are not readily compatible with a sequence of droplets in a microchannel. Therefore, in this section, we review methods for extracting the transported samples so that they can be subjected to more conventional analytical methods. Three strategies are listed below that serve to remove the continuous phase that separates the droplets. These methods leave droplets accessible for further detection [21,102]. They are: Evaporation of the continuous phase; an oleophilic membrane to selectively extract the continuous phase; and using negative pressure at a hydrophobic-hydrophilic interface with extract the continuous phase.

Some oils evaporate readily. In Figure 5a, the aqueous droplet phase is placed one by one on a Matrix Assisted Laser Desorption/Ionization (MALDI) plate together with the volatile perfluorinated oil (Perfuorohexane). Evaporation of both phases happens before the plate is loaded into the MALDI mass spectrometer. The evaporation time can be completed within less than $60 \mathrm{~s}$, or accelerated to less than $5 \mathrm{~s}$ with a flow of nitrogen gas [103]. It is also possible to form segmented flows (slugs) in liquid-gas microfluidic systems [104]. Here the water droplets can easily be isolated through evaporation; however, the droplets themselves are less stable [105].

The oil phase can be absorbed by an oleophilic and hydrophobic membrane. Figure $5 \mathrm{~b}$ shows that an oleophilic membrane made of Polytetrafluoroethylene (PTFE) was used to absorb and extract the continuous phase [106]. The aqueous phase was left suspended and placed one drop at a time on the hydrophilic part of a MALDI plate.

The oil phase can also be removed by negative pressure with the assistance of a hydrophobic-hydrophilic interface. Figure 5 c shows a microfluidic probe (MFP) system for writing chemical patterns $[7,107]$. This device uses segmented flows to allow different chemicals to be delivered through the same orifice. Droplets were generated using a standard T-junction, and transported to the probe tip. Negative pressure was provided at the oil removal channel of the probe's tip to remove the oil, while the small hydrophobic features function to retain the aqueous phase, due to the Laplace pressure. The water-based liquids are, thereby, expelled. 

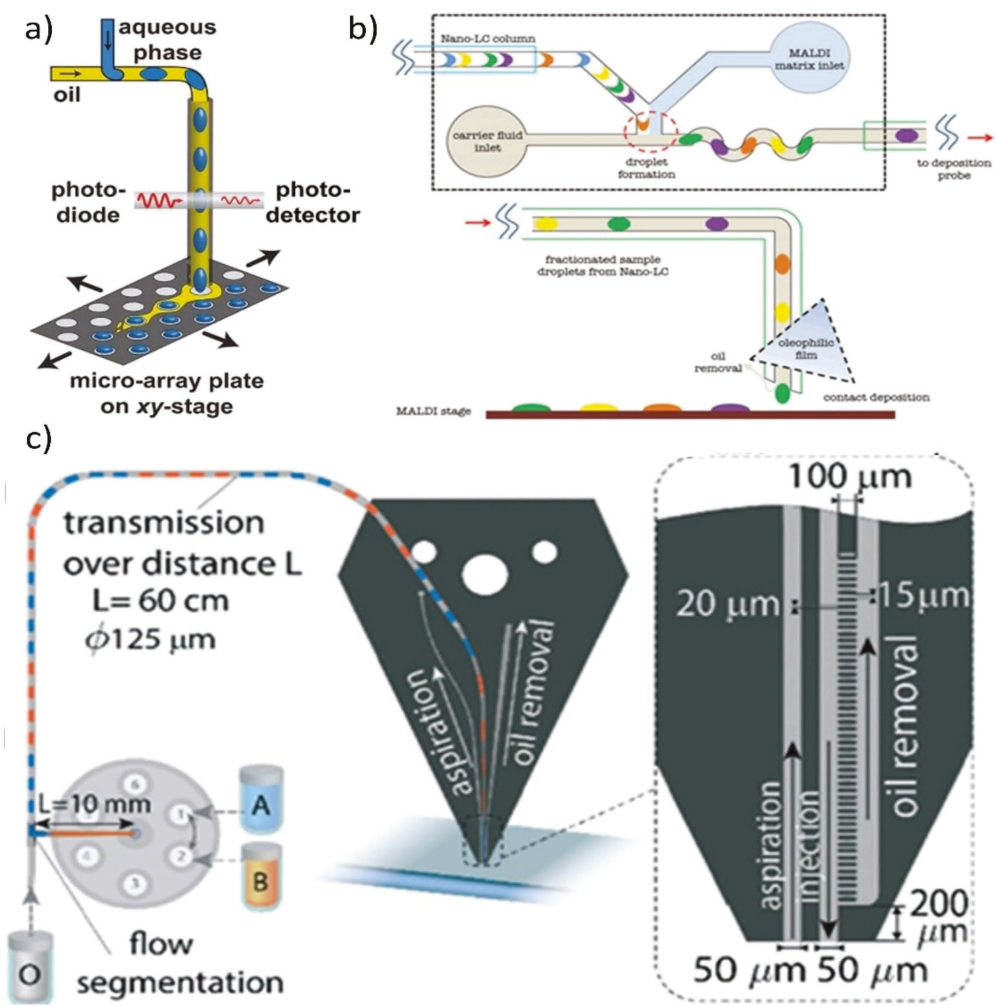

Figure 5. Off-chip water-based droplet extraction methods. (a) Schematic of droplet creation and spotting on a MALDI plate, where the hydrophobic carrier (oil) will evaporate quickly (Reprinted with permission from Reference [103]. Copyright 2013 American Chemical Society); (b) schematic of the device that used oleophilic oil film to extract the oil continuous phase and left the aqueous phase to the MALDI plate (Reprinted with permission from Reference [106]. Copyright 2013 PLOS ONE); (c) the developed microfluidic probe (MFP) system (Reprinted with permission from Reference [7]. Copyright 2016 Royal Society of Chemistry).

\section{Conclusions}

Point of care diagnostic devices reduce the distance that chemical signals must travel, and therefore, the dispersion, but for typical microchannels, it takes just millimeters for dispersion to be significant [69]. Furthermore, not all sensors can be brought to the sampling site. There have been many reviews of droplet-based microfluidics devices and droplet analysis methods. We focus on microfluidic slugs or droplets as a method of transporting chemical signals to overcome Taylor dispersion, and the devices use to capture those signals. We have surveyed examples of chemical signal sampling and delivery and classified them into three types: Aqueous phase sampling with downstream droplet formation; Preformed droplets for sampling; and Droplets formed near the analyte source. We pay particular attention to the temporal and spatial resolution of each system, and explain the features that limit each of these parameters. Strategies for downstream analysis have also been listed. We hope this review can help to broaden the use of droplet-based sampling probes for biochemical applications, enabling higher resolution study of fundamental processes.

Author Contributions: S.F. and D.W.I. collected and arranged the information; S.F., E.S. and D.W.I. wrote the paper. Conflicts of Interest: The authors declare no conflict of interest. 


\section{References}

1. Feng, S.L.; Dong, T. Applied technology in diaper-based UTI testing for elder people by using nitrite ion selective electrode. Mech. Eng. Mater. Inf. Technol. II 2014, 662, 225-228. [CrossRef]

2. Feng, S.L.; Roseng, L.E.; Dong, T. Quantitative detection of Escherichia coli and measurement of urinary tract infection diagnosis possibility by use of a portable, handheld sensor. In Proceedings of the 2015 IEEE International Symposium on Medical Measurements and Applications (MeMeA) Proceedings, Turin, Italy, 7-9 May 2015; pp. 586-589.

3. Lindholm-Sethson, B.; Nyström, J.; Geladi, P.; Koeppe, R.; Nelson, A.; Whitehouse, C. Are biosensor arrays in one membrane possible? A combination of multifrequency impedance measurements and chemometrics. Anal. Bioanal. Chem. 2003, 377, 478-485. [CrossRef] [PubMed]

4. Luka, G.; Ahmad, A.; Najjaran, H.; Alocilja, E.; DeRosa, M.; Wolthers, K.; Malki, A.; Aziz, H.; Althani, A.; Hoorfar, M. Microfluidics integrated biosensors: A leading technology towards lab-on-a-chip and sensing applications. Sensors 2015, 15, 30011-30031. [CrossRef] [PubMed]

5. Feng, S.L.; Dong, T. Design and characterization of a lab-on-chip for continuous bioluminescent measurements of ATP. In Proceedings of the 2014 IEEE International Symposium on Medical Measurements and Applications (MeMeA), Lisboa, Portugal, 11-12 June 2014; pp. 298-301.

6. Taylor, G. Dispersion of soluble matter in solvent flowing slowly through a Tube. Proc. R. Soc. Lond. Ser. A Math. Phys. Sci. 1953, 219, 186-203.

7. Ostromohov, N.; Bercovici, M.; Kaigala, G. Delivery of minimally dispersed liquid interfaces for sequential surface chemistry. Lab Chip 2016, 16, 3015-3023. [CrossRef] [PubMed]

8. Casadevall i Solvas, X.; deMallo, A. Droplet microfluidics: Recent developments and future applications. Chem. Commun. 2011, 47, 1936-1942. [CrossRef] [PubMed]

9. Wang, M.; Slaney, T.; Mabrouk, O.; Kennedy, R.T. Collection of nanoliter microdialysate fractions in plugs for off-line in vivo chemical monitoring with up to $2 \mathrm{~s}$ temporal resolution. J. Neurosci. Methods 2010, 190, $39-48$. [CrossRef]

10. Chen, D.; Du, W.; Liu, Y.; Liu, W.; Kuznetsov, A.; Mendez, F.E.; Philipson, L.H.; Ismagilov, R.F. The chemistrode: A droplet-based microfluidic device for stimulation and recording with high temporal, spatial, and chemical resolution. Proc. Natl. Acad. Sci. USA 2008, 105, 16843-16848. [CrossRef]

11. Wang, M.; Roman, G.T.; Schultz, K.; Jennings, C.; Kennedy, R.T. Improved temporal resolution for in vivo microdialysis by using segmented flow. Anal. Chem. 2008, 80, 5607-5615. [CrossRef]

12. Anna, S.L.; Bontoux, N.; Stone, H.A. Formation of dispersions using "flow focusing" in microchannels. Appl. Phys. Lett. 2003, 82, 364-366. [CrossRef]

13. Fidalgo, L.M.; Abell, C.; Huck, W.T. Surface-induced droplet fusion in microfluidic devices. Lab Chip 2007, 7, 984-986. [CrossRef] [PubMed]

14. Song, H.; Tice, J.D.; Ismagilov, R.F. A microfluidic system for controlling reaction networks in time. Angew. Chem. Int. Ed. 2003, 42, 768-772. [CrossRef] [PubMed]

15. Lorenz, R.M.; Edgar, J.S.; Jeffries, G.D.; Chiu, D.T. Microfluidic and optical systems for the on-demand generation and manipulation of single femtoliter-volume aqueous droplets. Anal. Chem. 2006, 78, 6433-6439. [CrossRef] [PubMed]

16. Garstecki, P.; Fuerstman, M.J.; Stone, H.A.; Whitesides, G.M. Formation of droplets and bubbles in a microfluidic T-junction-Scaling and mechanism of break-up. Lab Chip 2006, 6, 437-446. [CrossRef] [PubMed]

17. Link, D.R.; Grasland-Mongrain, E.; Duri, A.; Sarrazin, F.; Cheng, Z.; Cristobal, G.; Marquez, M.; Weitz, D.A. Electric control of droplets in microfluidic devices. Angew. Chem. Int. Ed. 2006, 45, 2556-2560. [CrossRef] [PubMed]

18. Sahoo, H.R.; Kralj, J.G.; Jensen, K.F. Multistep continuous-flow microchemical synthesis involving multiple reactions and separations. Angew. Chem. Int. Ed. 2007, 46, 5704-5708. [CrossRef] [PubMed]

19. Huebner, A.; Sharma, S.; Srisa-Art, M.; Hollfelder, F.; Edel, J.B. Microdroplets: A sea of applications? Lab Chip 2008, 8, 1244-1254. [CrossRef] [PubMed]

20. Song, H.; Chen, D.; Ismagilov, R. Reactions in droplets in microflulidic channels. Angew. Chem. Int. Ed. 2006, 45, 7336-7356. [CrossRef] [PubMed] 
21. Kaminski, T.; Garstecki, P. Controlled droplet microfluidic systems for multistep chemical and biological assays. Chem. Soc. Rev. 2017, 46, 6210-6226. [CrossRef]

22. Dressler, O.J.; Casadevall i Solvas, X.; deMello, A.J. Chemical and biological dynamics using droplet-based microfluidics. Ann. Rev. Anal. Chem. 2017, 10, 1-24. [CrossRef]

23. Damiati, S.; Kompella, U.; Damiati, S.; Kodzius, R. Microfluidic devices for drug delivery systems and drug screening. Genes 2018, 9, 103. [CrossRef] [PubMed]

24. Samiei, E.; Tabrizian, M.; Hoorfar, M. A review of digital microfluidics as portable platforms for lab-on a-chip applications. Lab Chip 2016, 16, 2376-2396. [CrossRef] [PubMed]

25. Chiu, D.T.; Di Carlo, D.; Doyle, P.S.; Hansen, C.; Maceiczyk, R.M.; Wootton, R.C. Small but perfectly formed? Successes, challenges, and opportunities for microfluidics in the chemical and biological sciences. Chem 2017, 2, 201-223. [CrossRef]

26. Robinson, D.L.; Hermans, A.; Seipel, A.T.; Wightman, R.M. Monitoring rapid chemical communication in the brain. Chem. Rev. 2008, 108, 2554-2584. [CrossRef] [PubMed]

27. Shou, M.; Ferrario, C.R.; Schultz, K.N.; Robinson, T.E.; Kennedy, R.T. Monitoring dopamine in vivo by microdialysis sampling and on-line CE-laser-induced fluorescence. Anal. Chem. 2006, 78, 6717-6725. [CrossRef] [PubMed]

28. Cesselin, F.; Hamon, M. Possible functional significance of the simultaneous release of several putative neurotransmitters by the same neuron. Annales D'endocrinologie 1984, 45, 207-213. [PubMed]

29. Hökfelt, T.; Bean, A.; Ceccatelli, S.; Dagerlind, A.; Elde, R.P.; Goldstein, M.; Melander, T.; Nicholas, A.P.; Pelto-Huikko, M. Neuropeptides and classical transmitters. Localization and interaction. Arzneimittel-Forschung 1992, 42, 196-201.

30. Bert, L.; Parrot, S.; Robert, F.; Desvignes, C.; Denoroy, L.; Suaud-Chagny, M.F.; Renaud, B. In vivo temporal sequence of rat striatal glutamate, aspartate and dopamine efflux during apomorphine, nomifensine, NMDA and PDC in situ administration. Neuropharmacology 2002, 43, 825-835. [CrossRef]

31. Song, P.; Mabrouk, O.S.; Hershey, N.D.; Kennedy, R.T. In vivo neurochemical monitoring using benzoyl chloride derivatization and liquid chromatography-mass spectrometry. Anal. Chem. 2011, 84, 412-419. [CrossRef]

32. Huynh, B.H.; Fogarty, B.A.; Martin, R.S.; Lunte, S.M. On-line coupling of microdialysis sampling with microchip-based capillary electrophoresis. Anal. Chem. 2004, 76, 6440-6447. [CrossRef]

33. Lada, M.W.; Vickroy, T.W.; Kennedy, R.T. High temporal resolution monitoring of glutamate and aspartate in vivo using microdialysis on-line with capillary electrophoresis with laser-induced fluorescence detection. Anal. Chem. 1997, 69, 4560-4565. [CrossRef] [PubMed]

34. Parrot, S.; Sauvinet, V.; Riban, V.; Depaulis, A.; Renaud, B.; Denoroy, L. High temporal resolution for in vivo monitoring of neurotransmitters in awake epileptic rats using brain microdialysis and capillary electrophoresis with laser-induced fluorescence detection. J. Neurosci. Methods 2004, 140, 29-38. [CrossRef] [PubMed]

35. Tucci, S.; Rada, P.; Sepúlveda, M.J.; Hernandez, L. Glutamate measured by 6-s resolution brain microdialysis: capillary electrophoretic and laser-induced fluorescence detection application. J. Chromatogr. B Biomed. Sci. Appl. 1997, 694, 343-349. [CrossRef]

36. Bert, L.; Robert, F.; Denoroy, L.; Stoppini, L.; Renaud, B. Enhanced temporal resolution for the microdialysis monitoring of catecholamines and excitatory amino acids using capillary electrophoresis with laser-induced fluorescence detection Analytical developments and in vitro validations. J. Chromatogr. A 1996, 755, 99-111. [CrossRef]

37. Hogan, B.L.; Lunte, S.M.; Stobaugh, J.F.; Lunte, C.E. Online coupling of in vivo microdialysis sampling with capillary electrophoresis. Anal. Chem. 1994, 66, 596-602. [CrossRef] [PubMed]

38. Song, P.; Hershey, N.D.; Mabrouk, O.S.; Slaney, T.R.; Kennedy, R.T. Mass spectrometry "sensor" for in vivo acetylcholine monitoring. Anal. Chem. 2012, 84, 4659-4664. [CrossRef] [PubMed]

39. Srisa-Art, M.; Dyson, E.C.; deMello, A.J.; Edel, J.B. Monitoring of real-time streptavidin-biotin binding kinetics using droplet microfluidics. Anal. Chem. 2008, 80, 7063-7067. [CrossRef]

40. Srinivasan, V.; Pamula, V.; Pollack, M.; Fair, R. A digital microfluidic biosensor for multianalyte detection. In Proceedings of the Sixteenth Annual International Conference on Micro Electro Mechanical Systems (MEMS-03), Kyoto, Japan, 23 January 2003; IEEE: Piscataway, NJ, USA, 2003; pp. 327-330. 
41. Malic, L.; Veres, T.; Tabrizian, M. Two-dimensional droplet-based surface plasmon resonance imaging using electrowetting-on-dielectric microfluidics. Lab Chip 2009, 9, 473-475. [CrossRef]

42. Malic, L.; Veres, T.; Tabrizian, M. Biochip functionalization using electrowetting-on-dielectric digital microfluidics for surface plasmon resonance imaging detection of DNA hybridization. Biosens. Bioelectr. 2009, 24, 2218-2224. [CrossRef]

43. Baret, J.-C. Surfactants in droplet-based microfluidics. Lab Chip 2012, 12, 422-433. [CrossRef]

44. Zheng, B.; Ismagilov, R.F. A microfluidic approach for screening submicroliter volumes against multiple reagents by using preformed arrays of nanoliter plugs in a three-phase liquid/liquid/gas flow. Angew. Chem. Int. Ed. 2005, 44, 2520-2523. [CrossRef] [PubMed]

45. Baroud, C.N.; Gallaire, F.; Dangla, R. Dynamics of microfluidic droplets. Lab Chip 2010, 10, $2032-2045$. [CrossRef] [PubMed]

46. Kamholz, A.E.; Weigl, B.H.; Finlayson, B.A.; Yager, P. Quantitative analysis of molecular interaction in a microfluidic channel: The T-sensor. Anal. Chem. 1999, 71, 5340-5347. [CrossRef] [PubMed]

47. Macounova, K.; Cabrera, C.R.; Holl, M.R.; Yager, P. Generation of natural pH gradients in microfluidic channels for use in isoelectric focusing. Anal. Chem. 2000, 72, 3745-3751. [CrossRef] [PubMed]

48. Yang, J.; Huang, Y.; Wang, X.B.; Becker, F.F.; Gascoyne, P.R.C. Differential analysis of human leukocytes by dielectrophoretic field-flow-fractionation. Biophys. J. 2000, 78, 2680-2689. [CrossRef]

49. Chen, Y.H.; Chen, S.H. Analysis of DNA fragments by microchip electrophoresis fabricated on poly(methyl methacrylate) substrates using a wire-imprinting method. Electrophoresis 2000, 21, 165-170. [CrossRef]

50. Ehrlich, D.J.; Matsudaira, P. Microfluidic devices for DNA analysis. Trends Biotechnol. 1999, 17, 315-319. [CrossRef]

51. Kamholz, A.E.; Yager, P. Theoretical analysis of molecular diffusion in pressure-driven laminar flow in microfluidic channels. Biophys. J. 2001, 80, 155-160. [CrossRef]

52. Chan, J.H.; Timperman, A.T.; Qin, D.; Aebersold, R. Microfabricated polymer devices for automated sample delivery of peptides for analysis by electrospray ionization tandem mass spectrometry. Anal. Chem. 1999, 71, 4437-4444. [CrossRef]

53. Li, J.J.; Kelly, J.F.; Chemushevich, I.; Harrison, D.J.; Thibault, P. Separation and identification of peptides from gel-isolated membrane proteins using a microfabricated device for combined capillary electrophoresis/nanoelectrospray mass spectrometry. Anal. Chem. 2000, 72, 599-609. [CrossRef]

54. Pinto, D.M.; Ning, Y.B.; Figeys, D. An enhanced microfluidic chip coupled to an electrospray Qstar mass spectrometer for protein identification. Electrophoresis 2000, 21, 181-190. [CrossRef]

55. Bernard, A.; Delamarche, E.; Schmid, H.; Michel, B.; Bosshard, H.R.; Biebuyck, H. Printing patterns of proteins. Langmuir 1998, 14, 2225-2229. [CrossRef]

56. Chiu, D.T.; Jeon, N.L.; Huang, S.; Kane, R.S.; Wargo, C.J.; Choi, I.S.; Ingber, D.E.; Whitesides, G.M. Patterned deposition of cells and proteins onto surfaces by using three-dimensional microfluidic systems. Proc. Natl. Acad. Sci. USA 2000, 97, 2408-2413. [CrossRef] [PubMed]

57. Folch, A.; Toner, M. Cellular micropatterns on biocompatible materials. Biotechnol. Prog. 1998, 14, 388-392. [CrossRef] [PubMed]

58. Kenis, P.J.A.; Ismagilov, R.F.; Whitesides, G.M. Microfabrication inside capillaries using multiphase laminar flow patterning. Science 1999, 285, 83-85. [CrossRef] [PubMed]

59. Datta, S.; Ghosal, S. Characterizing dispersion in microfluidic channels. Lab Chip 2009, 9, 2537-2550. [CrossRef] [PubMed]

60. Aris, R. On the dispersion of a solute in a fluid flowing through a tube. Proc. R. Soc. Lond. Ser. A Math. Phys. Sci. 1956, 235, 67-77.

61. Probstein, R.F. Physicochemical Hydrodynamics: An Introduction; John Wiley \& Sons: Hoboken, NJ, USA, 2005.

62. Kirby, B.J. Micro-and Nanoscale Fluid Mechanics: Transport in Microfluidic Devices; Cambridge University Press: Cambridge, UK, 2010.

63. Millington, D.; Norton, S.; Singh, R.; Sista, R.; Srinivasan, V.; Pamula, V. Digital microfluidics comes of age: High-throughput screening to bedside diagnostic testing for genetic disorders in newborns. Expert Rev. Mol. Diagn. 2018, 18, 701-712. [CrossRef]

64. Hou, H.W.; Bhattacharyya, R.P.; Hung, D.T.; Han, J. Direct detection and drug-resistance profiling of bacteremias using inertial microfluidics. Lab Chip 2015, 15, 2297-2307. [CrossRef] 
65. Thongkhao-On, K.; Kottegoda, S.; Pulido, J.S.; Shippy, S.A. Determination of amino acids in rat vitreous perfusates by capillary electrophoresis. Electrophoresis 2004, 25, 2978-2984. [CrossRef]

66. Pritchett, J.S.; Pulido, J.S.; Shippy, S.A. Measurement of region-specific nitrate levels of the posterior chamber of the rat eye using low-flow push-pull perfusion. Anal. Chem. 2008, 80, 5342-5349. [CrossRef] [PubMed]

67. Thongkhao-on, K.; Wirtshafter, D.; Shippy, S.A. Feeding specific glutamate surge in the rat lateral hypothalamus revealed by low-flow push-pull perfusion. Pharmacol. Biochem. Behav. 2008, 89, 591-597. [CrossRef] [PubMed]

68. Kottegoda, S.; Shaik, I.; Shippy, S.A. Demonstration of low flow push-pull perfusion. J. Neurosci. Methods 2002, 121, 93-101. [CrossRef]

69. Feng, S.L.; Liu, G.Z.; Jiang, L.M.; Zhu, Y.G.; Goldys, E.M.; Inglis, D.W. A microfluidic needle for sampling and delivery of chemical signals by segmented flows. Appl. Phys. Lett. 2017, 111. [CrossRef]

70. Slaney, T.R.; Nie, J.; Hershey, N.D.; Thwar, P.K.; Linderman, J.; Burns, M.A.; Kennedy, R.T. Push-pull perfusion sampling with segmented flow for high temporal and spatial resolution in vivo chemical monitoring. Anal. Chem. 2001, 83, 5207-5213. [CrossRef]

71. Berg, H.C. Random Walks in Biology; Princeton University Press: Princeton, NJ, USA, 1993.

72. Córcoles, E.P.; Boutelle, M.G. Biosensors and Invasive Monitoring in Clinical Applications; Springer: Berlin/Heidelberg, Germany, 2013.

73. Hurley, J.P.; Garrod, C. Principles of physics. Phys. Teach. 1978, 16, 408. [CrossRef]

74. Sanders, G.H.W.; Manz, A. Chip-based microsystems for genomic and proteomic analysis. Trac-Trends Anal. Chem. 2000, 19, 364-378. [CrossRef]

75. Verpoorte, E. Microfluidic chips for clinical and forensic analysis. Electrophoresis 2002, 23, 677-712. [CrossRef]

76. Rossier, J.; Reymond, F.; Michel, P.E. Polymer microfluidic chips for electrochemical and biochemical analyses. Electrophoresis 2002, 23, 858-867. [CrossRef]

77. Juncker, D.; Schmid, H.; Drechsler, U.; Wolf, H.; Wolf, M.; Michel, B.; de Rooij, N.; Delamarche, E. Autonomous microfluidic capillary system. Anal. Chem. 2002, 74, 6139-6144. [CrossRef]

78. Unger, M.A.; Chou, H.P.; Thorsen, T.; Scherer, A.; Quake, S.R. Monolithic microfabricated valves and pumps by multilayer soft lithography. Science 2000, 288, 113-116. [CrossRef] [PubMed]

79. Kennedy, R.T. Emerging trends in in vivo neurochemical monitoring by microdialysis. Curr. Opin. Chem. Biol. 2013, 17, 860-867. [CrossRef] [PubMed]

80. Timofeev, I.; Carpenter, K.L.H.; Nortje, J.; Al-Rawi, P.G.; O'Connell, M.T.; Czosnyka, M.; Smielewski, P.; Pickard, J.D.; Menon, D.K.; Kirkpatrick, P.J.; et al. Cerebral extracellular chemistry and outcome following traumatic brain injury: A microdialysis study of 223 patients. Brain 2011, 134, 484-494. [CrossRef] [PubMed]

81. Bossers, S.M.; de Boer, R.D.; Boer, C.; Peerdeman, S.M. The diagnostic accuracy of brain microdialysis during surgery: A qualitative systematic review. Acta Neurochir. 2013, 155, 345-353. [CrossRef] [PubMed]

82. Helmy, A.; Hutchinson, P. Is Cerebral Microdialysis a Clinical Tool? Springer: Vienna, Austria, 2013.

83. Weiss, D.J.; Lunte, C.E.; Lunte, S.M. In vivo microdialysis as a tool for monitoring pharmacokinetics. $\operatorname{Tr} A C$ Trends Anal. Chem. 2000, 19, 606-616. [CrossRef]

84. Hutchinson, P.J.; O'Connell, M.T.; Al-Rawi, P.G.; Maskell, L.B.; Kett-White, R.; Gupta, A.K.; Richards, H.K.; Hutchinson, D.B.; Kirkpatrick, P.J.; Pickard, J.D. Clinical cerebral microdialysis: A methodological study. J. Neurosurg. 2000, 93, 37-43. [CrossRef] [PubMed]

85. Wang, M.; Roman, G.T.; Perry, M.L.; Kennedy, R.T. Microfluidic chip for high efficiency electrophoretic analysis of segmented flow from a microdialysis probe and in vivo chemical monitoring. Anal. Chem. 2009, 81, 9072-9078. [CrossRef]

86. Croushore, C.A.; Sweedler, J.V. Microfluidic systems for studying neurotransmitters and neurotransmission. Lab Chip 2013, 13, 1666-1676. [CrossRef]

87. Fang, Q.; Shi, X.-T.; Sun, Y.-Q.; Fang, Z.-L. A flow injection microdialysis sampling chemiluminescence system for in vivo on-line monitoring of glucose in intravenous and subcutaneous tissue fluid microdialysates. Anal. Chem. 1997, 69, 3570-3577. [CrossRef]

88. Wang, M.; Hershey, N.D.; Mabrouk, O.S.; Kennedy, R.T. Collection, storage, and electrophoretic analysis of nanoliter microdialysis samples collected from awake animals in vivo. Anal. Bioanal. Chem. 2011, 400, 2013-2023. [CrossRef]

89. Myers, R. An improved push-pull cannula system for perfusing an isolated region of the brain. Physiol. Behav. 1970, 5, 243-246. [CrossRef] 
90. Myers, R.; Adell, A.; Lankford, M. Simultaneous comparison of cerebral dialysis and push-pull perfusion in the brain of rats: A critical review. Neurosci. Biobehav. Rev. 1998, 22, 371-387. [CrossRef]

91. Patterson, E.E., II; Pritchett, J.S.; Shippy, S.A. High temporal resolution coupling of low-flow push-pull perfusion to capillary electrophoresis for ascorbate analysis at the rat vitreoretinal interface. Analyst 2009, 134, 401-406. [CrossRef] [PubMed]

92. Cellar, N.A.; Kennedy, R.T. A capillary-PDMS hybrid chip for separations-based sensing of neurotransmitters in vivo. Lab Chip 2006, 6, 1205-1212. [CrossRef] [PubMed]

93. Cellar, N.A.; Burns, S.T.; Meiners, J.-C.; Chen, H.; Kennedy, R.T. Microfluidic chip for low-flow push-pull perfusion sampling in vivo with on-line analysis of amino acids. Anal. Chem. 2005, 77, 7067-7073. [CrossRef] [PubMed]

94. Van den Brink, F.T.G.; Phisonkunkasem, T.; Asthana, A.; Bomer, J.G.; van der Maagdenberg, A.M.J.M.; Tolner, E.A.; Odijk, M. A miniaturized push-pull-perfusion probe for few-second sampling of neurotransmitters in the mouse brain. Lab Chip 2019, 19, 1332-1343. [CrossRef] [PubMed]

95. Song, H.; Li, H.-W.; Munson, M.S.; Van Ha, T.G.; Ismagilov, R.F. On-chip titration of an anticoagulant argatroban and determination of the clotting time within whole blood or plasma using a plug-based microfluidic system. Anal. Chem. 2006, 78, 4839-4849. [CrossRef] [PubMed]

96. Chen, C.-F.; Drew, K.L. Droplet-based microdialysis-Concept, theory, and design considerations. J. Chromatogr. A 2008, 1209, 29-36. [CrossRef]

97. Sun, M.; Fang, Q. High-throughput sample introduction for droplet-based screening with an on-chip integrated sampling probe and slotted-vial array. Lab Chip 2010, 10, 2864-2868. [CrossRef]

98. Gielen, F.; Burzska, T.; Van Vliet, L.; Butz, M.; Damborsky, J.; Prokop, Z.; Hollfelder, F. Interfacing microwells with nanoliter compartments: A sampler generating high-resolution concentration gradients for quantitative biochemical analyses in droplets. Anal. Chem. 2014, 87, 624-632. [CrossRef]

99. Feng, S.; Clement, S.; Zhu, Y.; Goldys, E.M.; Inglis, D.W. Microfabricated needle for hydrogen peroxide detection. RSC Adv. 2019, 9, 18176-18181. [CrossRef]

100. Feng, S.L.; Nguyen, M.N.; Inglis, D.W. Microfluidic droplet extraction by hydrophilic membrane. Micromachines 2017, 8, 331. [CrossRef] [PubMed]

101. Zhu, Y.; Fang, Q. Analytical detection techniques for droplet microfluidics-A review. Anal. Chim. Acta 2013, 787, 24-35. [CrossRef] [PubMed]

102. El Debs, B.; Utharala, R.; Balyasnikova, I.V.; Griffiths, A.D.; Merten, C.A. Functional single-cell hybridoma screening using droplet-based microfluidics. Proc. Natl. Acad. Sci. USA 2012, 109, 11570-11575. [CrossRef]

103. Küster, S.K.; Fagerer, S.R.; Verboket, P.E.; Eyer, K.; Jefimovs, K.; Zenobi, R.; Dittrich, P.S. Interfacing droplet microfluidics with matrix-assisted laser desorption/ionization mass spectrometry: Label-free content analysis of single droplets. Anal. Chem. 2013, 85, 1285-1289. [CrossRef] [PubMed]

104. Tirandazi, P. Droplet Formation and Entrainment in Liquid-Gas Microfluidic Systems. Master's Thesis, Northeastern University, Boston, MA, USA, 2017.

105. Martín-Banderas, L.; Flores-Mosquera, M.; Riesco-Chueca, P.; Rodrígez-Gill, A.; Cebolla, Á.; Chávez, S.; Gañán-Calvo, A.M. Flow focusing: A versatile technology to produce size-controlled and specific-morphology microparticles. Small 2005, 1, 688-692. [CrossRef]

106. Pereira, F.; Niu, X. A nano LC-MALDI mass spectrometry droplet interface for the analysis of complex protein samples. PLoS ONE 2013, 8, e63087. [CrossRef]

107. Van Kooten, X.F.; Autebert, J.; Kaigala, G.V. Passive removal of immiscible spacers from segmented flows in a microfluidic probe. Appl. Phys. Lett. 2015, 106, 074102. [CrossRef] 
MDPI

St. Alban-Anlage 66

4052 Basel

Switzerland

Tel. +41616837734

Fax +41 613028918

www.mdpi.com

Biosensors Editorial Office

E-mail: biosensors@mdpi.com www.mdpi.com/journal/biosensors

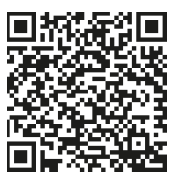



MDPI

St. Alban-Anlage 66

4052 Basel

Switzerland

Tel: +41 616837734

Fax: +41 613028918

www.mdpi.com 